Portland State University

PDXScholar

\title{
Combat Experiences, Iso-strain, and Sleep Quality Affect Symptoms of Posttraumatic Stress among Working Post-9/11 Veterans
}

Gilbert Patrick Brady Jr. Portland State University

\section{Recommended Citation}

Brady, Gilbert Patrick Jr., "Combat Experiences, Iso-strain, and Sleep Quality Affect Symptoms of Posttraumatic Stress among Working Post-9/11 Veterans" (2017). Dissertations and Theses. Paper 3776. https://doi.org/10.15760/etd.5660

This Thesis is brought to you for free and open access. It has been accepted for inclusion in Dissertations and Theses by an authorized administrator of PDXScholar. Please contact us if we can make this document more accessible: pdxscholar@pdx.edu. 
Combat Experiences, Iso-strain, and Sleep Quality Affect Symptoms of Posttraumatic Stress among Working Post-9/11 Veterans

by

Gilbert Patrick Brady, Jr.

A thesis submitted in partial fulfillment of the requirements for the degree of

\author{
Master of Science \\ in \\ Psychology
}

Thesis committee:

Leslie B. Hammer, Chair

Keith L. Kaufman

Joel S. Steele

Portland State University

2017 
(C) 2017 Gilbert Patrick Brady, Jr. 


\begin{abstract}
Despite the growing need, prior research on how the civilian work domain may affect posttraumatic stress is scarce. Moreover, few if any studies have investigated how perceptions of one's job and insomnia may shape traumatic stress symptoms, postcombat. Presently, about 4 million Americans have served in the "Global War on Terrorism," including nearly 1 million reservists. By contrast, 8.7 million Americans served in the Vietnam War: over twice the number of U.S. military personnel who have fought since 9/11. Surprisingly, combat experiences alone do not explain the majority of posttraumatic stress disorder cases, even after multiple deployments, suggesting the presence of moderators of the stressor-strain conceptual model. Based in occupational stress theories of allostatic load, job demands-resources, strain, and social support, this thesis applied frequentist and Bayesian analytical strategies to investigate the psychological experiences and occupational health of three subgroups of combat veterans. In the present study, sleep quality and a job situation characterized by isolated, demanding civilian work with low decision authority (i.e., "iso-strain") were hypothesized to moderate the effect of combat experiences on posttraumatic stress. As part of the larger SERVe project, survey data were collected among a baseline sample $(N=382)$ of post-9/11 veterans living in the Pacific Northwest. The research question of whether greater perceived psychosocial stressors among active reservists driving that group's higher self-reported levels of poor sleep, iso-strain, and posttraumatic stress was unfounded. Overall, however, the central
\end{abstract}


hypotheses of the simple effects of iso-strain and sleep quality on posttraumatic stress following combat were significant. 


\section{Acknowledgements}

I would like to thank Dr. Leslie B. Hammer, my thesis chair, for her curious mentorship. Her boundless interest in the influence of the civilian work milieu in shaping reintegration and posttraumatic stress among working veterans has been a continuous source of inspiration to me. The insights and resources she has provided throughout this process, along with a grant funded by the U.S. Department of Defense (DoD, PI Hammer: \#W81XWH-13-2-0020), and the participation of my brothers and sisters in the National Guard were fundamental to my completion of this thesis. Additionally, I would like to thank my academic mentors Drs. Keith L. Kaufman, Joel S. Steele, Todd E. Bodner, Liu-Qin Yang, Kathleen F. Carlson, David E. Caughlin, Thomas J. Kindermann, Ellen A. Skinner, Donald M. Truxillo, Bill Griesar, Ian H. Dinwoodie, Jason T. Newsom, Karen M. Chenier, Charlotte Fritz, and my graduate school cohort, classmates, friends and all around academic pal James L. Delaney for all of their feedback and advice. In sum, colleagues, old and new, not only improved the quality of my thesis but also helped me to develop into a better researcher. Finally, and most especially, I am forever grateful and indebted to my loving and wonderful wife, Rebecca Caruba-Brady, and joyous son, Rowyn Brady, for all their years and hours of support and the many personal sacrifices they have made and endured in allowing me to achieve this goal. 


\section{Table of Contents}

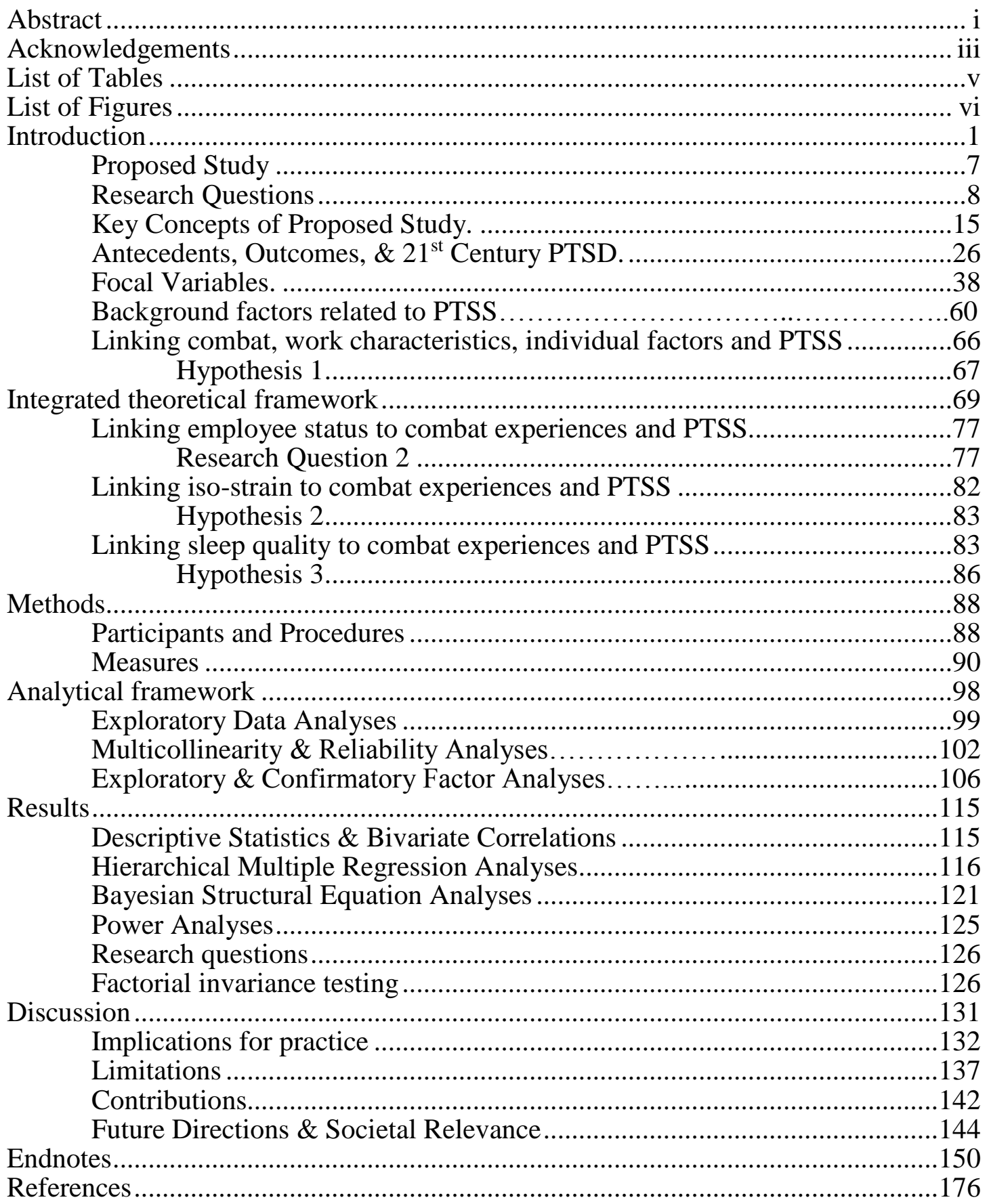




\section{List of Tables}

Table 1: Descriptive statistics of SERVe combat veteran employees................... 154

Table 2: Exploratory Factor Analysis of Combat Experiences Scale................156

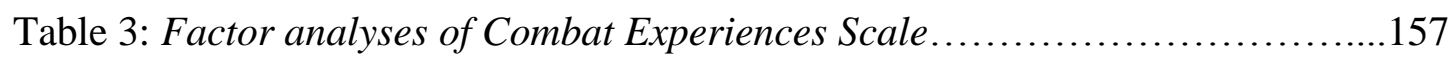

Table 4: Correlation \& scale reliabilities matrix of all study variables.............159

Table 5: Hierarchical multiple regression \& parameter estimates..................160

Table 5(b): Hierarchical multiple regression \& parameter estimates..................161

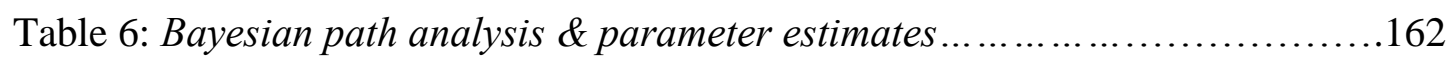

Table 6(b): Bayesian path analysis \& parameter estimates ..........................163

Table 7: Summary of Combat Experiences invariance test....................................172

Table 8: Summary of PC-PCL invariance test .................................. 173 


\section{List of Figures}

Figure 1. Proposed conceptual model....................................151

Figure 2. Changes from DSM-IV to DSM-5: Clinical criteria for PTSD.............152

Figure 3. Predictions from the JD-R model based on interaction effects............153

Figure 4. Hypothesis\#2: Iso-strain will moderate CES on PTSS...................155

Figure 5. Hypothesis\#3 Sleep Quality will moderate CES on PTSS ...............155

Figure 6. Four-factor formative MIMC Combat Experiences Scale..................158

Figure 7. Hours slept per night by veteran employee group................................. 164

Figure 8 . Iso-strain by veteran employee group............................. 164

Figure 9. PTSS by veteran employee group............................... 165

Figure 10. Combat Experiences by veteran employee group..................... 165

Figure 11 . Sleep quality by veteran employee group.......................... 166

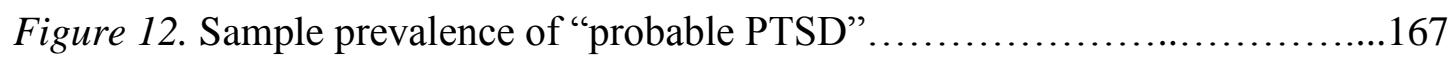

Figure 13. Combat Experiences $x$ Iso-strain 2-way interaction graph...............168

Figure 14. Combat Experiences x Sleep quality 2-way interaction graph............169

Figure 15. Bayesian path model of CES, Iso-strain, \& Sleep Quality on PTSS......170

Figure 16. Single-group $F$-test power analysis using five tested predictors...........171

Figure 17. Demographics: post-9/11 veterans by age group...................174

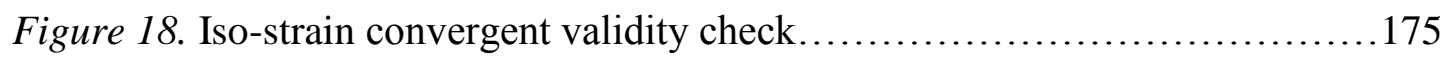




\section{Introduction}

More than a million working-age veterans are projected to leave the armed services by 2020 (Office of the Chairman of the Joint Chiefs of Staff [OCJC], 2014), presenting an ongoing national priority to reintegrate this generation of former military men and women successfully back into society while they are still in the prime of their lives. This national priority is rooted in federal policies aimed at stimulating hiring demand by providing tax breaks to organizations that employ veterans (McDonough \& Sperling, 2013). Interestingly, decades of research indicate that more than two-thirds of veterans complete their military service, including multiple combat tours, and return to their families, communities and society without significant functional impairment (e.g., Bonanno, Mancini, Horton, Powell, LeardMann, Boyko, \& Smith et al., 2012; Kulka, Schlenger, Fairbank, Hough, Jordan, Marmar, \& Weiss, 1988; McNally, 2007). Nonetheless, high-profile initiatives such as President Barack Obama's tax-incentive driven "Vow to Hire a Hero" program reflect societal concerns that veterans represent a historically disadvantaged group in need of greater economic supports (McDonough \& Sperling, 2013).

Historical accounts of maladjustment among returning U.S. military personnel date to at least the Revolutionary and Civil wars in the $18^{\text {th }}$ and $19^{\text {th }}$ centuries (Da Costa, 1851; Resch, 1999). Yet, it would take a late $20^{\text {th }}$ Century political movement amid chaotic scenes of veterans joining and forming protest groups over the U.S. war in Southeast Asia to prompt elected officials to take the social welfare of veterans more seriously (Chamber II, 1999, p.536). Specifically, images of disenfranchised veterans of 
the Vietnam War (1963-1975), many experiencing unemployment, homelessness (Lewin, 1987), “criminal justice involvement” (Kulka, Schlenger, Fairbank, Hough, Jordan, Marmar, \& Weiss, 1990, p.183), and apparent posttraumatic stress reactions compelled Congress to mandate an investigation of the social and psychological problems related to military service during Vietnam (e.g., Kulka et al., 1988, 1990b). More than forty years later, the enormity of challenges facing today's all-volunteer professional military remains an emotionally charged political issue despite active service members and veterans of the $21^{\text {st }}$ Century having record levels of "concentrated programmed support" (Shurr, Lunney, Bovin, \& Marx, 2009, p.728). For example, a Government Accountability Office (GAO) report identified 186 programs run by either the U.S. Department of Defense (DoD) or U.S. Department of Veterans Affairs (VA) specifically aimed at helping Gulf War II (i.e., "post-9/11”) military personnel transition to civilian life. Of these programs, 99 focused on dealing with the effects of combat and 18 on employment assistance. (GAO, 2014). However, low participation rates ( 2\%) with on-the-job-training and apprenticeship programs have undermined their effectiveness (GAO, 2015).

Beyond large-scale stimulus-based efforts to stabilize the employment situation of veterans (e.g., tax incentives for businesses to hire former service members), peerreviewed scholarship and federal data indicate that without optimal, evidence-based workplace interventions (e.g., psychoeducational trainings for the leaders of companies) government incentives to employ former service members will be insufficient to retain a sizeable fraction (18\% to 41\%) of them on the job. Accordingly, over the course of 
the highly competitive, post-recession U.S. labor market (2008-2016), labor force participation rates (BLS, 2017) for Gulf War II veterans (83.6\% to 81.5\%) have declined. Hence, there is a critical need to identify modifiable risk factors within the civilian work domain that may reduce incidence of debilitating mental health disorders such as depression (e.g., Lerner, Adler, Rogers, Chang, Lapitsky, McLaughlin, \& Reed, 2010) and posttraumatic stress (e.g., Adler et al., 2011; Pietrzak, Goldstein, Malley, Johnson, \& Southwick, 2009) to keep veterans employed and succeeding on the job.

Post-9/11 generation. Since the terrorist attacks of September $11^{\text {th }}, 2001$, over 4 million men and women, representing less than 1\% of all Americans (Taylor, 2011), have served on active duty and about 3.0 million have deployed into or around the Middle East (Tanielian \& Ramchand, 2015; U.S. Bureau of Labor Statistics [BLS], 2016; DoD, 2015; VA, 2016). Like the U.S. workforce in general (e.g., Byars-Winston, Fouad, \& Wen, 2015) today's era of war veterans are the most demographically diverse in American history (Sayer, Carlson, \& Frazier, 2014). Compared to those who served in the decades long war in Vietnam (1963-1975), where over $90 \%$ of the 3.4 million who deployed to Southeast Asia were white, today's active duty military is more racially diverse with over a third of those having deployed since 2003 identifying as persons of color (e.g., Sayer et al., 2014). According to a national poll (Washington Post/Kaiser Family Foundation, 2013) of Iraq and Afghanistan service members $(N=819)$, half of all post-9/11 veterans are Southerners, a third hold a college degree, and a majority hail from the nation's rural areas. In addition, at least 673,000 women (16.5\%) have now completed a tour of active duty (BLS, 2017; Sayer et al., 2014, p.35). 
This relatively small pool of diverse wartime veterans also includes 1 million-plus military reservists (BLS, 2016; DoD, 2015; Tanielian \& Ramchand, 2015; VA, 2015). However, while some white papers suggest that the modern U.S. military is also more economically diverse (e.g., Dubner, 2008; Watkins, Shrek, 2008), young post-9/11 veterans (18 to 24$)$ still "face high rates of unemployment, poverty and homelessness" (Perl, 2015). In sum, not since the colonial era, when "few middle-class Americans bore arms in the Continental Army (Ferling, 2010, p.4)," have the experiences and consequences related to modern wartime military service been shared by fewer Americans serving among a more representative troupe of their fellow countrymen.

Military service and occupational health. In addition to the demanding nature of modern military service - where multiple deployments, women serving in combat roles, and the sobering likelihood of surviving previously deadly battlefield injuries (e.g., Izzo, 2013) from such mechanical forces as roadside bombs inflicting trauma across multiple bodily organs and psychological systems (i.e., polytrauma) have all become the "new norm"- reactions to war-related violence and persistent life threat may be exacerbated by a raft of common reintegration stressors (e.g., Sayer et al., 2014, Taber \& Hurley, 2009). As a foundational construct of Industrial and Organizational (I-O) Psychology psychosocial stressors have been defined as "environmental, social, and organizational conditions" that may be "perceived as harmful or threatening" (Heaney, 2011). These stressors may range from: the demands of one's job (e.g., work overload, time pressure); financial problems; marital distress; inconsistent or ambiguous social support; sleep difficulties; length of time between deployments, and work-family 
conflict (Adler et al., 2011, Heaney, 2011, Hobfoll, 2012) to name but a few. Further, either alone or in concert, experiencing prolonged psychological stressors at work consisting of high job demands and low job control (i.e., degree of employee authority and influence over the work process) may result in perceived job strain(s), or the potential "psychological, physical, and behavioral outcomes of negatively perceived stress" (Mullen \& Kelloway, 2011). Job strain may become particularly unhealthy (e.g., Mather, Bergstrom, Blom, Svedberg, 2015) in terms of sick leave due to mental disorders in work situations characterized by iso-strain (i.e., high demands, low control, and low social support at work). Perceived job strain may also manifest as anxiety, depression, indecisiveness (e.g., Mullen \& Kelloway, 2011), insomnia (e.g. Hobfoll, 1989/1998) and other changes in physical and mental health (e.g., Kivimäki, Nyberg, Batty, Fransson, Heikkilä, Alfredsson, et al., 2012; Stansfeld, Shipley, Head, \& Fuhrer, 2012; Wang, Mykletun, Møyner, Øverland, Henderson, Stansfeld, et al., 2014), and family stability (e.g., Gold, Taft, Keehn, King, King, \& Samper, 2007). Further, prolonged exposure to job strain(s) may lead to burnout (Demerouti, Bakker, Nachreiner, Schaufeli, 2001) and even breakdown (Bakker \& Demerouti, 2007, p.313). For example, in a study of rookie police officers a "routine work environment" mediated the effect of critical incidents on posttraumatic stress disorder or "PTSD" (Maguen, Metzler, McCaslin, Inslicht, Henn-Haase, Neylan et al., 2009). Consequently, the developmental pathway between experiences of combat and later manifestations of PTSD has a strong theoretical and empirical foundation (e.g., Laposa, Alden, \& Fullerton, 2003) through the influences of the civilian work milieu. 
Over the last fourteen years scholarly works in epidemiology and clinical psychology have repeatedly linked the effects of Gulf War-era II (2003-present) military service with increased post-deployment prevalence of mental, physical and behavioral health problems (e.g., Sayer et al., 2014) as well as serious financial hardships, legal troubles, family violence, incarceration, homelessness, suicide, and employment-related difficulties (e.g., Jakupcak, Cook, Imel, Fontana, Rosenheck, \& McFall, 2009; Wilcox, Oh, Redmond, Chicas, Hassan, Lee \& Ell, 2015). Surprisingly, a broad sampling of the OHP and I-O literature reveals a conspicuous gap in research addressing the need to extend decades of clinical findings by developing and testing new psychological interventions to improve the prospects of veterans entering and reentering the modern workplace. Seven years ago, in a study of occupational functioning among post-9/11 veterans using VA healthcare, Sayer et al. (2010) reported that $25 \%$ to $41 \%$ of combat veterans of Iraq and Afghanistan had some level of productivity difficulties (e.g., keeping a job, completing daily tasks for home, work, or school). More recently, in a critical review of post-deployment studies that illustrated how veterans exposed to combat differentially acquired PTSD, diagnosis of PTSD among post-9/11 combat veterans was associated with a five-fold rate of "problems finding or keeping a job" compared with combat veterans without PTSD (Sayer et al., 2014). Again, this line of largely clinical work from within the nation's primary healthcare system for military veterans (the U.S. Department of Veterans Affairs) is consistent with the finding that combat exposure and combat experiences, in general, are not necessarily causative of PTSD. 
In fact, since the mapping of the human genome in 2003 (e.g., NIH, 2016), a wealth of new discoveries in biopsychosocial research indicates that the interplay of environmental factors and qualifying traumatic stressors (e.g., combat, rape, vehicular accidents) appears to explain, over and above individual differences such as one's genetic makeup, greater variance in symptoms of posttraumatic stress (Wolf, Mitchell, Koenen, \& Miller, 2014). As surprising as it may be, and for as long as healthy young Americans have gone to and returned from war with a multitude of physical and psychological traumas and readjustment challenges, a recent review of the work reintegration literature concluded that there is "limited knowledge about how to reintegrate people with mental disorders" (Van Til, Fikretoglu, Pranger, Patten, Wang, Wong et al., 2013). Thus, the role of the civilian work domain in shaping posttraumatic stress symptoms ("PTSS") is an underexplored area ripe for investigation by social scientists and occupational health practitioners motivated to improve the long neglected health, economic stability, and lifelong well-being of veterans entering, reentering, or returning to work, their families, and society.

Proposed study. The proposed theoretical model (Figure 1.) illustrates the hypothesized effects of organizational (i.e., iso-strain) and individual (i.e., sleep quality) predictors on the relationship between experiences of combat and PTSS. Other substantive questions related to the potential impacts of larger organizational influences within America's district forms of military cultures (e.g., air, sea, and land) and varying forms of civilian work organization (i.e., public, private, public-private) or civilian occupations (e.g., blue vs. white collar) were not investigated. As no unified theory of 
military-to-civilian work reintegration presently exists (e.g., Schultz, Chlebak, \&

Stewart, 2016; Van Til et al., 2013) this thesis study applied an integrated theoretical

approach. Prior industrial, organizational, clinical and biopsychosocial theories, as well as scholarly military and occupational health perspectives and findings on "stressorstrain relationships" among military veterans and civilian workers in high-risk occupations informed conceptual distinctions and predicted associations among focal variables of interest. In addition, the question of how these hypothesized relations differed among three subgroups (i.e., Active Reservists, Separated Active Component, Separated Reserve Component) of participating veteran employees, endorsing a prior military affiliation and at least one combat experience, was also explored.

Research Question \#1: Are measures of combat experiences and posttraumatic stress sufficiently invariant among veteran employee subgroups to provide confidence in any significant findings of group differences?

Research Question \#2: Does moderation (i.e., ISO x CES, Sleep Quality x CES) analyses reveal significant differences on measures of job stress (i.e., ISO) and well-being (i.e., Sleep Quality) among subgroups (i.e., Active Reservists, Separated Active, Separated Reserve) of veteran employees?

A secondary aim of the current work was to propose a candidate variable (i.e., isostrain) for extending the nomological network of veteran reintegration into civil society. This goal involved the development, application, and testing of an analytical strategy integrating frequentist and Bayesian theoretical frameworks that may be ideally suited to a model-building approach that utilizes estimates of parameters from "prior knowledge" (e.g., meta-analyses, literature reviews, expert opinion) to detect subtle effects with 'high dimensional' data'. Such small and/or complex and subtle effects may 
be due to the greater prevalence of such attributes as multiple deployments, polytrauma, and the greater demographic diversity of the United States' armed forces, all factors uniquely associated with post-9/11 military service. Thus, detecting complex effects with high dimensional data may be more difficult using traditional statistical methods such as multiple regression and path analysis with ordinary least squares or maximum likelihood estimation, all of which assume multivariate normality (e.g., Mallick \& Nengiun, 2013; Schmidt, 2011).

For instance, in their landmark National Vietnam Veterans Readjustment Study (NVVRS), Kulka and colleagues relied on classic multivariate regression techniques to test the independent effects of warzone experiences on mental health symptoms among returning Vietnam veterans (Kulka et al., 1990). Fundamentally, multiple regression analysis assumes perfect measurement of all manifest variables (Crocker \& Algina, 2008). Accordingly, a subsequent reanalysis of Vietnam-era data, derived from the NVVRS's then-gold-standard methodological approach (i.e., combining veteran selfreports with structured clinical interviews), cross-validated these records against later access U.S. military archives. After failing to account for numerous claims of veterans experiencing qualifying "Criterion A" stressor(s) in actual combat, Dohrenwend and colleagues downwardly readjusted the original NVVRS prevalence estimate of PTSD by $40 \%$ (Dohrenwend, Turner, Turse, Adams, Koenen, \& Marshall, 2007). Nonetheless, this latest revision of the psychological effects of military service during Vietnam still estimated that nearly 19\% met criteria for PTSD (Dohrenwend et al., 2007). Interestingly, despite technological advances in lifesaving battlefield medicine, personal 
armor, and combat intelligence systems, the latest meta-analyses (Fulton, Calhoun, Wagner, Schry, Hair, Feeling et al., 2015) of military data (2007-2013) estimated prevalence of PTSD at $23 \%$ among post-9/11 veterans.

The current thesis study aimed to overcome traditional constraints and limitations (e.g., Koopman, Howe, Hollenbeck, \& Sin, 2015) on studying subgroup differences with small samples (i.e., $N<80$ ), nonnormally distributed focal variables, and potentially nonlinear associations among predictors of PTSS by utilizing advanced analytical modeling frameworks (e.g., Bayesian SEM) and computer probability sampling algorithms (e.g., Markov Chain Monte Carlo). Specifically, Bayesian SEM (BSEM) parameter estimates from Markov Chain Monte Carlo (MCMC) resampling algorithms may calculate numerical approximations of multi-dimensional integrals (Koopman et al., 2015; Muthén \& Asparouhov, 2011). In addition to improving upon estimation of population parameters, application of such methods as BSEM addressed a major challenge in the present study: obtaining stable point estimates of population parameters on a range-restricted sample of working combat veterans seven years, on average, postdeployment. Such sample-based constraints necessitated a multistep analytical approach to account for maximum measurement and sample error variance.

\section{Extending clinical and military research into the U.S. workforce. Formerly} classified as an "anxiety disorder," prior to the American Psychiatric Association's (APA) current professional clinical diagnostic manual (APA, 2013) of mental diseases, four years ago PTSD was reclassified as a "trauma and stressor-related disorder" (Figure 2.) predictive of later work impairment (Adler et al., 2011; APA, 2013, pp.271-280) with 
unemployment on occasion predicting PTSD severity over and above depression (Anderson, 2014). While studies among veterans and workers in high-risk occupations (e.g., first responders, farmers), have documented the incapacitating effects (e.g., Adler et al., 2011) of psychiatric status (e.g., depression, PTSD) on occupational functioning (e.g., mental-interpersonal demands, time management, output), reviews of the evidence to date (e.g., Dewa \& Lin, 2000; Kessler, Ormel, Demler \& Stang, 2003; Lim, Sanderson, \& Andrews, 2000) reveal that psychiatric disorders mostly impair rather than fully debilitate employees' functional ability.

Therefore, the harmful impact of mental health on work-related outcomes offers opportunities for intervention at various ecological levels (Bronfenbrenner, 1986). Besides responding to calls for organizational researchers to address the well-being of wage-earning and vulnerable workers and thereby narrow the academic-practitioner gap (e.g., Bergman \& Jean, 2016), this thesis aimed to extend current military and clinical efforts to lower the risk for PTSD (e.g., Adler et al., 2011; Troxel, Shih, Pedersen, Geyer, Fisher, Griffin et al., 2015) into the civilian domain by examining psychosocial factors related to the health and well-being of workers in high risk (e.g., Cahill \& Landsbergis, 1996) and lower skilled occupations (e.g. Milner, Spittal, Pirkis, \& LaMontagne, 2013) in order to better understand the psychological strengths, needs, and vulnerabilities of civilian workers with military backgrounds (Troxel et al., 2015). As the role of the civilian work domain in shaping posttraumatic stress is the central focus of this study, a summary of how PTSD's latest reconfiguration by the American Psychiatric Association (APA) aligns with the professional goals of I-O and 
Occupational Health Psychology (OHP) may help illuminate the importance of this complex and still evolving biopsychosocial construct for work psychologists.

\section{Relevance of OHP to military-to-civilian work reintegration. For over a}

century, I-O psychologists have made major contributions to the recruitment, selection, and placement of military recruits (e.g., Lindsay, n.d.). Presently, there exists a large and growing demand for professional work psychologists to refocus their expertise on improving the lives of the hundreds of thousands of working-age men and women who exit the military each year (OCJC, 2014) and seek employment in civilian organizations that may be wary of hiring them (e.g., Kukla, Rattray, Salyers, 2015) and vary widely in appropriate responsiveness to their particular strengths and challenges (e.g., McCreary, Peach, Blais, \& Fikretoglu, 2014). To this end, within the field of OHP there is also an emerging consensus that psychological problems need not reach full clinical criteria to still have detrimental effects on interpersonal and occupational functioning (e.g., Adler et al., 2011; Breslau, Lucia, \& Davis, 2004). In addition, prior studies (e.g., Purtle, Lynn, \& Malik, 2016; Overman \& Leonard, 2010; Zoroya, 2013) suggest that the biased attitudes of key organizational decision-makers toward veteran job candidates may contribute to the maintenance of prejudicial barriers to recruiting, hiring, placing, developing, and advancing former servicemembers and veteran employees (e.g., Kukla et al, 2015). Paradoxically, Hollywood producers and other American cultural impresarios have portrayed veterans as both indefatigably heroic (e.g., GI Joe, John Wayne) and more frequently since Vietnam as potentially dangerous (e.g., Hoit, 2012) and "damaged by their service" (e.g., Kleykamp \& Hipes, 2015). In oversimplifying the 
diversity of veterans and veteran reintegration challenges "pop culture" caricatures may also foment additional harmful organizational stressors (e.g., stigma, role ambiguity) contributing to veteran unemployment (e.g., Anderson, 2014) and work impairment (e.g., Britt, Green-Shortridge, Brink, Nguyen, Hoge, \& Castro, 2008) through perpetuation of stereotypes, implicit biases, and veteran acquiescence to stigma (i.e., "self-stigma") resulting in reduced self-efficacy and mental illness, (e.g., Corrigan, Watson, \& Barr, 2006). In the case of PTSD these and other workplace stressors may affect the job performance dimensions of: time management; interpersonal functioning; completion of tasks; assignments; and overall productivity as measured by output (e.g., Adler et al., 2011).

Accordingly, a goal of occupational health research is the identification of risk and protective factors within the organizational environment (e.g., work overload, coworker support, stigma) that may offer discrete targets for interventions designed to decrease either actual job stressors (e.g., hours worked), or the harmful effects of perceived job stressors (e.g., stigma) by increasing the actual or perceived availability of psychosocial buffers (Quick \& Tetrick, 2011) of stressor-strain dynamics such as the availability of organizational resources (e.g., supervisor support) as may be provided by social capital (Centers for Disease Control \& Prevention [CDC], 2013). For any given society, pools of human resources and community characteristics are forms of "social capital" (CDC, 2013). Social capital may include "communal time", "recreation", "social networking" and "civic engagement" and other activities (e.g., work team "go green" energy conservation contests) that form "social bonds". Social capital is a determinant of health 
and well-being for populations and individuals. Multilevel models of the link between social capital at work and health impairment predict higher odds for impaired health among employees in work groups low on social capital (Oksanen, Kouvonen, Kivimaki, Pentti, Virtanen, Linna, \& Vahtera, 2008). Further, the effect of positive social participation on self-rated health (SRH), along with cognitive and physical health, may differ by veteran subgroups with participation in group activities being positively associated with better SRH (Choi, DiNitto, \& Marti, 2015). Other outcomes of social capital at work include both self-reported and physician-diagnosed depression and treatment for depression (e.g., Kouvonen, Oksanen, Vahtera, Vaananen, De Vogli, \& Elovainio et al., 2008). Workplace social capital may also be contextually patterned by demographic, employment changes, and work unit size (Oksanen, Kawachi, Kouvonen, Takao, Suzuki, Virtanen et al., 2013) that may present unique cognitive-affective challenges for some veterans with emerging symptoms (e.g., negative alterations in cognitions and mood) of posttraumatic stress (Liberzon \& Abelson, 2016). In sum, these findings reveal how variation in workplace social capital and social support at work (e.g., Ganster, Fusilier, \& Mayes, 1986) can influence mental and physical health, which may impact veteran reintegration and socialization into the workplace. In turn, organizational socialization and the direction of work engagement among veteran employees may be related to their perceptions of their professional self-efficacy, absence of workplace stigma, appropriate resources (e.g., relevant job training, support from supervisors), and the benefits of health and well-being from social capital and social support as provided by participating in positive workplace social networks. By 
either reducing actual stressors or the perceptions of them (e.g., by increasing job control, work resources, social capital) psychologists may improve the chances of atrisk workers with emerging psychological symptoms of strain (e.g., insomnia), who aspire to reintegrate into the civilian workplace, to avoid developing debilitating mental disorders (e.g., depression, PTSD) that may limit their chances of maintaining productive work lives.

\section{Key concepts of proposed study}

Military reintegration. Reintegration refers to the process of military personnel returning to personal, organizational, and societal roles after deployment (Currie, Day, \& Kelloway, 2011). Within the military psychological literature, reintegration typically covers the first 30 to 180 days post-deployment and includes both a return to work and family life (Currie et al., 2011). From a civilian perspective work is a form of social behavior imbrued within a complex socialization processes (Neff, 1985). Five decades of studies show that, on average, at least two-thirds of U.S. veterans reintegrate successfully (e.g., Fulton et al., 2015; Hoge et al., 2006; IOM, 2013) and many easily transfer marketable knowledge, skills, abilities and other characteristics (KSOs) acquired through certain military occupational specialties (e.g., flywheel mechanic, meteorologist, combat surgeon) directly to gainful civilian employment (De Groat \& Crowley, 2013). Nevertheless, only recently has serious scientific inquiry into the phenomena of civilian work reintegration with military populations (e.g., Brady \& Hammer, 2013; Kulka et al., 2015; McCreary et al., 2014) offered theories and critiques to explain how the reintegration process unfolds, especially for the large minority of 
former military personnel who consistently experience significant difficulty adapting to the civilian labor market while a majority of their fellow war comrades appear to thrive (e.g., Bonanno et al., 2012; Kulka et al., 1990).

Early reintegration phase: bias and stigma. A review of the research literature suggests that the period immediately following military separation from active duty is the most difficult and risky as far as behavioral health and social functioning (e.g., Adler et al., 2011; Killgore, Cotting, Thomas, Cox, McGurk, Vo et al., 2008; Shea et al., 2010). After separating from the military about 1 in 5 of today's veterans experience difficulty holding a job and many experience family difficulties (Adler et al., 2011). Perhaps even more disconcerting are the tens of thousands of service members who separate from the military under other than honorable conditions (e.g., Morin, 2011), leaving many of them ineligible for veterans' benefits (VA, 2015) and at higher risk for homelessness and suicide (Kessler, Warner, Ivany, Petukhova, Rose, Bromet et al., 2015). In addition to the extraordinary burden placed on the fraction of Americans who have served in uniform over the last sixteen years, common misconceptions of veterans and blatant hiring stigma from public and private employers, and low and ambiguous support from supervisors, coworkers (e.g., Riviere, Kendall-Robbins, McGurk, Castro, \& Hoge et al., 2011) and the general public, even for those with jobs, appears as common today as it was two generations ago. Take, for example, the following except from a report by the National Advisory Council on Vocational Education:

Statistics show that (veterans) have a higher unemployment rate than nonveterans, and that their unemployment persists longer. Factors contributing to this condition are the economic slump, a difficult readjustment 
process, lack of skills, lack of vocational counseling, inadequate government programs, and hostility toward the Vietnam veteran (Davenport, 1972).

What is most striking about Davenport's report on the vocational status and economic prospects of returning Vietnam veterans is how closely it resembles more recent analyses of the perceptions and attitudes of prospective employers toward the present generation of returning service members seeking civilian jobs within an unstable post-recessionary America where the uneven rate of recovery since 2008 has resulted in historic levels (U.S. Census, 2016) of poverty, forty-four years later (Ashkenas \& Parlapiano, 2014). Survey-based research probing the perceptions and attitudes of human resource professionals toward post-9/11 veterans reveals how the legacy of Vietnam and other unpopular American wars continue to bias the decision-making processes of civilian employers (e.g., Kukla et al., 2015; Stone \& Stone, 2015). In 2010, for example, the Society for Human Resource Management (SHRM) polled a sample ( $N=148)$ of its membership (Overman, \& Leonard, 2010). Results showed that more than 2 in 3 hiring managers endorsed concerns with hiring either a newly reintegrated veteran or an active member of the National Guard or Reserve. Further, $46 \%$ endorsed safety concerns over bringing "PTSD" or "other mental health issues" into the workplace with more than 3 in 4 holding negative biases, stereotypes ${ }^{\mathrm{ii}}$ and doubts over the adaptability to the civilian workplace culture of veteran job applicants short on civilian employment histories, relevant education or job skills and long on post-9/11 military experiences (e.g., Overman \& Leonard, 2010). Unsurprisingly, a subsequent corporate-sponsored survey (Prudential, 2012) of Active and Reserve Component post-9/11 veterans $(N=2,453)$ reported that $64 \%$ 
endorsed having difficulties adjusting to civilian life with $69 \%$ revealing that "finding a job" was their "greatest challenge". While societal attitudes toward veterans are often positive (e.g., disciplined, team players, patriotic, exhibiting leadership) research based in disability models (e.g., Stone \& Stone, 2015) reveals that the historic employment problems of ex-military service members are associated with the persistent hiring stigmas and prejudices of human resource professionals toward people with perceived disabilities, including veterans.

Veteran entry into civilian labor market and early PTSD. As a group, veterans frequently take a civilian job for the first time after leaving military service (e.g., Van Til et al., 2013), or when returning to their civilian job after a prolonged absence (i.e., 9 months or longer). Often, service members return home from deployments with newly acquired prodromal symptoms (e.g., nightmares) that place them at risk for the acquisition of PTSD (APA, 2013; Raskind, Peskind, Hoff, Hart, Holmes, Warren, et al., 2007). Though little to no research with veterans has investigated the role of the workplace in shaping PTSD, nor any of its prodromal indicators (e.g., "belief in a foreshortened future"), a meta-regression of 24 prospective studies with multiple measurement points revealed that nearly 1 in 4 (24.8\%) PTSD cases followed a course of delayed onset (Smid, Mooren, van der Mast, Gersons, \& Kleber, 2009). Of these, military combat exposure, Western heritage, and lower cumulative incidence of PTSD were linked with delayed onset, and subthreshold PTSD at baseline elevated risk of delayed onset of full-blown PTSD (Smid et al., 2009). Additionally, core PTSD symptoms such as avoidance/numbing and hyperarousal may predict occupational 
functioning while reexperiencing may not (Shea et al., 2010). Also, in a review of seven prospective studies, Dutch researchers (Nieuwenhuijsen, Bruinvels, \& Frings-Dressen, 2010) reported that work situations characterized by high iso-strain and high effortreward imbalance "predicted stress-related disorders". Since the prevailing clinical model predicts that most veterans with full PTSD (i.e., onset $\geq 30$ days of experiencing a qualifying stressor, APA, 2013, p.271) would be too impaired to maintain fulltime employment postreintegration and meta-analyses reveal that combat exposures are a risk factor for delayed onset of PTSD, which occurs in about $25 \%$ of all cases, logic suggests that some percentage of combat veterans are entering the civilian labor market at higher risk for delayed onset of PTSD under certain environmental conditions such as job strain.

At first glance, delayed onset of PTSD among war veterans appears to fit neatly within the Stress-Diathesis Hypothesis (e.g., Beard, 1881; Ingram \& Luxton, 2005). The hypothesis proposes that idiosyncratic vulnerabilities contribute to development of psychopathology such as "trauma and stressor-related disorders" (e.g., APA, 2013, p.271) that may manifest well after exposure to combat (e.g., PTSD) under particular adverse environmental conditions. These confluences of distal and proximal processes are common experiences among former post-9/11 service members reintegrating into a highly competitive, economically unstable post-recessionary job market with high rates of unemployment among young veterans. Such a progression would also be predicted by a "sleeper model" (e.g., Theorell, Jodko, Konarski, \& Norbeck, 1994) whereby absence of the stressor does not eliminate manifestations of strain. While useful for 
identifying those with preexisting vulnerabilities for illness under adverse environmental conditions, Stress-Diathesis Theory is limited by an emphasis on individual and genetic liabilities that do not explain how mental illness impacts other functional domains over the lifespan as may be exhibited by iso-strain situations predictive of "stress-related disorders" (Nieuwenhuijsen et al., 2010) à la delayed onset PTSD (e.g., Smid et al., 2009). Though research is still vague on which specific civilian work conditions, job characteristics, and individual factors may adversely affect core PTSD symptoms among veteran employees and other workers in high-risk occupations (e.g., EMTs), and therefore compromise their healthy adaptation to the civilian workforce, what is known about the phenomena of reintegration among prior cohorts, such as before and after Vietnam, is that healthy reintegration includes successful adaptation to the workplace (Van Til et al., 2013).

Socialization and fit within a multilevel HR model. Improving the fit between an organization and its employees is a goal of I-O psychology and a premise of organizational change models (Landy \& Conte, 2013). Socialization is the process of how new employees become cognizant of the values, policies, and procedures of an organization, which "starts at the recruiting stage" when the organization informs job candidates about its mission, goals, and ideals. Socialization occurs through the interactions between newcomers and human resources, managers, and other support staff and departments (Landy \& Conte, 2013, p.571). With regard to socialization for veteran newcomers, evidence from organizational-based research reveals that persistent barriers in the form of biases and prejudices exist for veterans entering the $21^{\text {st }}$ Century 
workforce that may negatively impact their socialization, organizational commitment, job security, and long-term health. How this troubling state of affairs arose in a country with arguably the most powerful military force in history is currently a topic of much speculative debate among academics, veterans, and citizens.

One line of speculation (e.g., De Groat et al., 2013) on the success or failure of military veterans entering and re-entering the civilian labor market argues that "negative attitudes toward work" are associated with increased strain, turnover intentions, and decreased affective commitment (e.g., Blais, Thompson, \& McCreary, 2009; Currie et al., 2011). The negative work attitudes (TNWA) view proposes that the unrealistic expectations of finding emotionally satisfying civilian work ultimately clashes with the perceived low social prestige of many menial available jobs and the relatively meager financial rewards of comparatively mundane civilian work (i.e., "low value work"). Such common cognitions and perceptions are often held by socially naïve veterans lacking marketable skills, post-secondary education and poor civilian work histories (DeGroat \& Crowley, 2013; OCJCS, 2014). Poor civilian work histories coupled with low educational attainment, these military reintegration experts argue, fail to communicate synergy with the requisites of competitive civilian jobs based on an emerging multilevel human resource model (e.g., DeGroat \& Crowley, 2013; Ployhart \& Moliterno, 2011) that favors employees whose KSOs are perceived as contributing to organizational resources (e.g., receptivity to, sharing in, and the creation of new learning and knowledge) that can be leveraged for strategic economic gain (Ployhart et al., 2011). Convincing as the "negative work attitudes" perspective may be such one- 
dimensional constructs cannot explain the mechanisms underlying observable behavioral interactions and unobservable psychophysiological dynamics linking the work environment, where the socialization process unfolds, with the trajectory of stressrelated symptoms manifested by the veteran newcomer or returning veteran employee.

Feeling as information. By contrast, the "feeling as information hypothesis" asserts that feelings act as a "judgment-simplifying heuristic device" (Seo, Barrett, \& Bartunek, 2004, p.6) whereby individuals utilize their transitory feeling states to formulate impressionistic judgments (e.g., Schwarz, 1990; Schwarz \& Clore, 1983). Studies of the influence of affective experiences in driving work motivation reveal a complex process for managers and supervisors to consider when assessing the interplay of emotions, motivation, and goal-setting among workers (Seo et al., 2004). For instance, where negative affect (NA) such as anxiety may serve adaptive functions in situations when future events (e.g., deadlines) can impact entire businesses; positive affect (PA) may be more adaptive in situations requiring flexibility and creativity. However, left unchecked PA may facilitate irrational exuberance and overconfidence in persisting in unproductive behaviors requiring change, and global affect may influence "mood congruence recall effect" whereby people remember past events reflective of "their affective state at the time of recall" (Seo et al., 2004, p.7). Likewise, according to the 'feeling-as-information' hypothesis, people susceptible to highly activated feeling states (e.g., such as negative cognitions, mood, and hyperarousal in PTSD) may engage in "mood maintenance" and "mood repair" (Seo et al., 2004, p.11). That is, to alleviate negative feeling states (e.g., anxiety) and associated cognitions (e.g., dread) veterans 
may disengage from productive work by emotion focused-coping (Lazarus \& Folkman, 1984) manifesting as withdrawal and avoidance behaviors. As a hallmark symptom cluster associated with PTSD, avoidance behaviors are predictive of work performance (e.g., APA, 2013, pp.271-280), and joblessness (e.g., APA, 2013, pp.271-280) with unemployment potentially predicting severity of PTSD (e.g., Anderson, 2014).

Withdrawal and/or avoidance behaviors may act to decrease negative feeling states by way of psychologically devaluing the "mechanistic execution" of requisite tasks related to goal-oriented work activities (e.g., Demerouti et al., 2001; Seo et al., 2004, p.11). Job demands and resources. Additional theoretical processes potentially contributing to the behavioral direction and course of posttraumatic symptoms during and after the veteran employee socialization process are given by the Job DemandsResources (JD-R) model (Demerouti \& Bakker, 2011). Among the most empirically supported work stress theories, the JD-R proposed two dynamics - the health impairment process and performance protection strategies - that underlie psychological, physiological, behavioral, and motivational phenomena (Demerouti \& Bakker, 2011). Both the health impairment process and performance protection strategies may explain how classic PTSD symptoms associated with avoidance behaviors, activated by chronic psychosocial and physical job demands (e.g., stigma, overwork) in the absence of perceived or available organizational resources (e.g., coworker support, job control, feedback), both initially protect but ultimately impair the well-being, work performance, and ultimate organizational retention of the veteran employee. The JD-R's "dual processes" provide discrete theoretical mechanisms 
underlying worker health, well-being, and motivation that have predicted employee burnout (Demerouti et al., 2001) and work engagement (Bakker, Hakanen, Demerouti, $\&$ Xanthopoulou, 2007). For job-seeking veterans with prodromal symptoms (e.g., nightmares, foreshortened future orientations) specific to PTSD the influence of intense affective experiences associated with maladaptation to the civilian labor market and altered work motivation would predict that realization of one's employment deficits (i.e., nontransferable military job skills) may arouse negative emotions and secondary cognitive appraisals (Lazarus \& Folkman, 1984) of incompetence and feelings of worthlessness (Warr, 1987) following appraisal of negative feedback in the form of hiring rejection in pursuit of suitable civilian work. If such a cycle of negative reinforcement from prospective employers, who perceive an applicant's history of military service as dangerous and job relevant skill sets as lacking, eventuates in unemployment, underemployment, and/or low skill/low value employment prolonged negative affect (e.g., shame, rejection, humiliation, worthlessness) may erode professional efficacy and sense of purpose (e.g, Kukla et al., 2015). In turn, such effects may generalize into disaffection for civilian work (e.g., Demerouti et al., 2001) and alienation toward society. Such human reactions to one's posttraumatic environment may activate symptoms predictive of PTSD (e.g., DeGroat \& Crowley, 2013; OCJCS, 2014; Kline, Ciccone, Falca-Dodson, Black, Losonczy, 2011; Kukla et al., 2015).

TNWA and Culture Shock. The Negative Work Attitudes (TNWA) perspective also implies that for some young veterans who joined the military directly out of high school, and became accustomed to performing highly specialized and valued military 
occupational specialties (MOSs), reentry into the civilian labor market and taking a civilian job for the first time since exiting military service may be experienced as a form of culture shock. (De Groat et al., 2013; Taylor, 2011). Technically, from an experiential perspective, military reintegration is a form of reassimilation back into civilian life that may result in a form of acculturation stress (Ruesch, Jacobson \& Loeb, 1949) similar to the psychosocial stress experienced by émigrés struggling to maintain their culture of origin while also adapting to and participating in a new country with strange customs, symbols, norms, social expectations, and cultural values that may challenge one's identity and, by doing so, erode one's mental health (e.g., Berry, 1997; Duca, 2016; Ruesch et al., 1949).

Consequently, preventing delayed onset PTSD by modifying aspects of the organizational culture of the modern workplace (e.g., work redesign, team cohesion building, increasing supervisor, coworker, and senior leadership support, job crafting, creating veteran affinity groups) stands to benefit all of society. Lowered stress, strain, psychiatric and psychosomatic complaints, illnesses, and decreased interpersonal conflict and group-level perceptions of systemic prejudices among otherwise competent veteran and disabled workers - whose positive work engagement may hinge on creating more supportive team-oriented work environments - could benefit more than only a minority of employees. Changing dimensions of the culture of work may also benefit organizational stakeholders (e.g., supervisors, employees, coworkers, shareholders, and customers/clients) who otherwise espouse public support for employing veterans and 
differently abled workers and have invested considerable organizational, personal, and societal resources into doing so.

\section{Antecedents, outcomes, and 21 ${ }^{\text {st }}$ Century PTSD}

Background, selection, and contextual antecedents. In their first of a kind national study of Vietnam veterans (NVVRS), Kulka and colleagues (1990) tested whether dispositional, demographic, military-related, or selection factors affected prevalence of PTSD. An aim of the NVVRS was to estimate whether the experiences of war itself could be sufficiently isolated from other nuisance variables to conclude that levels of warzone stress were the main cause of the post-deployment acquisition of PTSD. In conclusion, the authors found that though background characteristics were related to current PTSD, and may have therefore rendered combat theatre veterans more vulnerable, even after accounting for predisposing selection factors, prevalence rates of PTSD were "much higher among theatre veterans" and "exposure to war-zone stress in Vietnam" played "a significant role in determining" who "has PTSD today" (Kulka et al., 1990, p.85). At the time, these findings fit a behaviorist paradigm as proposed by Mowrer's (1960) two-factor integrated theory of fear conditioning and learning, which was later extended by cognitive-affective models of PTSD (e.g., Ehlers \& Clark, 2000) that proposed how peri and posttraumatic thought, affect, and memories mediated and moderated PTSD (e.g., Brewin \& Holmes, 2003).

Contextual Processing Model and PTSD. By contrast, the state of science since Kulka et al. (1990) and Ehlers and Clark (2000), which includes the genetic and neuroscience revolutions of the present century, along with new understandings of gene 
$\mathrm{x}$ environment and brain $\mathrm{x}$ context interactions, has uncovered more complex and indirect pathways to acquiring full-blown, multidimensional PTSD. Just recently, for example, Liberzon and Abelson (2016) published experimental evidence from animal models and human imaging studies supporting their proposed Context-Processing Model (CPM). Briefly, the CPM implicates environmental context, inflexible cuefocused behavioral reactivity, and changes in neural networks connecting the medial prefrontal cortex, thalamus, hippocampus, and locus coeruleus, which are all theoretically involved in "contextual memory" and information processing. The CPM predicts how deficit contextual memory processing can lead to inappropriate cueelicited fear-associated responses and, paradoxically in PTSD patients, a failure to detect "danger" and "warning" signals leading to "reckless behaviors", "retraumatization" and SNS activation (Liberzon \& Abelson, 2016, pp.21-22). The neural networks implicated in the CPM also underlie the physiology of mammalian stress and adaptation that in concert with the hypothalamus-pituitary-adrenal (HPA) axis are related to the JD-R's health impairment process (Bakker \& Demerouti, 2007).

Accordingly, finding meaningful work and meaningful work relationships in a novel organizational context where performance management systems are maintained by such impersonal means as computers that track individual profit-driven benchmarks, which are then systematically rewarded by supervisors and managers (e.g., Spector, 2006, p.99) rather than linking job and work team goals with organizational objectives through engaging leadership (e.g., Vance, pp.16-17), may be disorienting and stressful for a new or returning communal-minded veteran employee. Evidence from experimental studies 
of contextual-processing (e.g., Liberzon \& Abelson, 2016) adds support to the JD-R's "duel processes" mechanisms and organizational-level explanations from a biopsychosocial perspective on the potential for interactions and reciprocal relations among the work situation, individual cognition, affect and biobehaviorial correlates of PTSS ranging from hypervigilance, recklessness, inappropriate anger, avoidance, and nocturnal re-experiencing phenomena like nightmares.

Post-9/11 veteran subcultures. A consistent finding over at least the last two major U.S. war eras is the robust association of homelessness and PTSD (e.g., Institute of Medicine (IOM), 2013, p.338-340; Kulka et al., 1988). Preliminary results from a VA analysis of post-9/11 data suggest that homeless veterans of the current era are more likely to have a diagnosis of PTSD (67\% vs. 13-18\%) compared with veterans of prior eras (IOM, 2013). In the post-9/11 military literature, well-controlled longitudinal studies appear after cross-sectional studies. Among military subgroups serving in Iraq or Afghanistan both study designs have uncovered differential risks and prevalence rates of PTSD (e.g., Bliese, Wright, Adler, Thomas, \& Hoge, 2007; Milliken et al., 2007). Studies that evaluated troops at two postdeployment time points (Bliese et al., 2007) revealed overall increases in PTSD. More specifically, by $\sim 6$ months postdeployment Milliken and colleagues (2007) found that post-9/11 Army Reserve and National Guard troops screened higher for PTSD (52.2\%) than Active Duty Army soldiers (40.8\%). The consistency of differential post-deployment trajectories of prevalence of PTSD among veteran subgroups underscores that the shared burden and consequences of a nation at perpetual war now includes all uniformed members 
regardless of their component of military service. However, not all burdens of war are necessarily equally shared even among those serving in them. Subgroup differences on post-deployment trajectories of PTSD may be reflections of differential re-assimilation stressors including 'Culture Shock' at the subcultural level as experienced by the $\sim 818,000$ men and women (DoD, 2016) currently serving in the National Guard and Reserve Component.

National Guard and Reserve Component. The post-9/11 ascendance of a generation of National Guard and Reserve Component veterans to meet that war fighting demands of prosecuting the 16-year long 'Global War on Terrorism' was a direct result of changes in Pentagon policies (e.g., Brown, 2007; Kirk, 2004) following September $11^{\text {th }}$ (e.g., Booth $\&$ Lederer, 2012a). In the aftermath of the $9 / 11$ attacks by associates of the terrorist group al-Qaeda, the reliance on reservists by policymakers to fulfill repeated deployments alongside active duty forces amounted to a reorganization and realignment of the historic role and purpose of the Reserve Component. By 2010, the Department of Defense reported that separating Reserve and National Guard troops represented 44\% of U.S. war veterans who had served in Iraq or Afghanistan (Sayer et al., 2014). Within two years, the Army Reserve and Army National Guard had supplied the Active Component Army with over 45\% of its soldiers (National Guard Bureau, 2012). This organizational restructuring was codified in the U.S. Army Reserve's new personnel plan (“Army Force Generation”) that forecast a deployment tempo of one-toone-and-half-years for every five years at home (Morrow, 2011). In addition to traditional stateside duties (e.g., firefighting, natural/manmade disaster relief, 
backcountry rescues), the expansion of the role of the National Guard (e.g., Atkins, 2013) and other military reservists since 2001 has evolved into fulfilling repeated deployments to foreign combat zones in-between returning to unstable post-deployment civilian milieus characterized by high unemployment and underemployment (e.g., Kline et al., 2011; Lakhani \& Fugita, 1993).

Besides experiencing higher rates of depression and PTSD and lower structural, formal, and informal supports coming off deployments, by 2010 suicide rates (Griffith \& Vaitkus, 2013) among Army National Guard soldiers (31 per 100,000) exceeded the active Army (25 per 100,000), Army Reserve (24 per 100,000), and the civilian ageadjusted rate (20.3 per 100,000). Most recently, after numerous large studies found that pre-enlistment childhood abuse and birth cohort factors were bigger risk factors for suicide among military personnel (e.g., Afifi, Taillieu, Zamorksi, Turner, Cheung, \& Sareen, 2016; Griffith \& Bryan, 2016) than deployment-related factors, Shen, Cunha, Williams (2016) reported a time-varying association with suicide linking "exposure to deployment, mental health diagnoses, and other stressful life events". In the present study, the second research question probed whether higher levels of psychosocial stressors among active reserve veteran employees, who must balance competing civilian and military job demands, resulted in relatively higher levels of iso-strain, poorer sleep, and PTSS compared with separated active and reserve veteran employees. By necessity, proposing to investigate group differences requires a theory of prediction of finding them. 
Allostatic load and group differences on strain. Occupational stress theories such as Sterling and Eyer's (1988) "Allostatic Load Model" (ALT) describe how an "allostatic state" indicates "a chronic over-activation of regulatory systems" that may "alter set points to various effector systems" (e.g., cardiovascular, neuroendocrine, hypothalamic-pituitary-adrenal axis) (e.g., Quick \& Terrick, 2011, p.34). Essentially, chronic over-activation of physiological regulatory systems may produce "wear and tear throughout the body" otherwise known as "allostatic load" (Sapolsky, 2004, p.14). By contrast, "allostasis" (i.e., literally, "stability through change") references physiological response systems that override homeostatic systems in response to environmental demands and anticipated demands that may be perceived as emanating from the psychosocial environment as originally proposed by Karasek (1979). The notion of "allostatic state" and the downstream effects of "allostatic load" maps onto a general thesis of allostatic-driven "work overload" such that multiple competing job demands (e.g., workload, work pressure, scheduling conflicts) from two or more concomitant work roles (e.g., military flywheel mechanic and civilian electrician) may be, without adequate recovery time between civilian and military work shifts or deployments, more intensely perceived and thus motivate endorsement of higher levels of "hard work" and "role conflict" and other job demand-related stressors (e.g., Karasek, 1979) among "multiple job holders" (e.g., moonlighters, active military reservists) as proposed by Betts (2002), Hirsch and Husain (2014), and Sliter and Boyd (2014). Therefore, active reservist veteran participants in SERVe were expected to endorse higher levels of isostrain, poor sleep quality, and PTSS at baseline. 
Empirical support of the expectation of finding veteran subgroup differences on measures of stressors and strain and mental health symptoms come from populationlevel studies of subgroups of returning combat veterans that indicate a differential 'allostatic state' may evolve during the reintegration and postreintegration phases. For example, nine years ago, in the first large $(N=88,235)$ longitudinal post-9/11 study of mental health and military reintegration, National Guard veterans returning from Iraq reported more than double (42.4\% v. $20.3 \%$ ) the prevalence of depression and PTSD symptoms within the first six months of returning home compared with Iraq veterans returning to active-duty (Milliken et al., 2007). Subsequent large studies have also reported similar differential group effects. Using administrative healthcare data ( $N=289,328)$, Seal, Metzler, Gima, Bertenthal, Maguen, and Marmar (2009) reported mental health diagnoses among veterans seeking VA care (2002-2008) increased more over time among reservists than active duty veterans. While different post-deployment experiences may explain subgroup differences on measures of mental health and wellbeing, the increased reliance on the National Guard and Reserve to fulfill overseas missions has resulted in record levels of multiple deployments for both the Active and Reserve components (e.g., Atkins, 2013). Over four years ago, for example, at least 115,000 Army National Guard had completed two or more tours of duty since 9/11 (National Guard Bureau, 2013). Unlike the fulltime "regulars" that they frequently deploy with, however, deployed reservists tend to be older and many leave family and civilian careers and jobs to fulfill yearlong tours of duty that may include combat and combat support missions (e.g., Bonanno et al., 2012, Riviere et al., 2011, Sayer et al., 
2014). While their active duty counterparts typically live in garrison or in affordable housing between deployments -on well-resourced posts, ports, and airbases - reservists typically report to their local armories one weekend a month while spending the remainder of their days living in their communities (Riviere et al., 2011).

Conservation of Resources Theory, "recycling," and veteran health. The effects of military policies requiring multiple deployments for American troops has resulted in a de facto "recycling phenomena" with increased risk for polytrauma and retraumatization (e.g., Taber \& Hurley, 2009). These cumulative "recycling" effects now extend well beyond the airbases, Army posts, and naval ports, where active duty members live and work, to affect the more than 3,300 U.S. communities where hundreds of thousands of post-9/11 reserve military personnel and veterans also exist (National Guard Bureau, 2012). According to ALT, post-deployment differences on mental and physical health would be expected among veterans differing on socioeconomic status, social capital, and social support. However, ALT does not address future societal liabilities from U.S. military policies requiring multiple deployments among the fraction of military personnel who are healthy enough to be "recycled" (i.e., redeployed). Such a person-focused model does not account for the unequal allocation of vital community reintegration resources among surviving veterans back home.

Structurally, coming off deployments, many veteran reservists reintegrate into a system of progressive "resource loss spirals" (e.g., Hobfoll, 1989; Hobfoll, Vinokur, Pierce, Lewandowski-Romps, Hobfoll, \& Galea, 2012). To start, compared with typical 
Active Duty military serving within the five major branches, reservist veterans experience lower day-to-day social support from their fellow war comrades and platoon mates upon returning home. This loss of "day-to-day support" from those with whom they potentially shared confusing, traumatic, unresolved and ultimately meaningful war experiences arises from the organizational practice of reservists living "off-post" as "citizen-soldiers". While ALT focuses on the individual effects of stressors, the grouplevel psychological effects of such differential experiences of the shared loss of vital post-deployment supports and resources are particularly salient to "Conservation of Resources" (COR) theory (Hobfoll, 1989).

As more sophisticated stressor-strain theories were required to explain the newly identified phenomena going on inside and outside the human body (e.g., Seyle's “General Adaptation Syndrome”, 1950), COR sought to advance the modern scientific understanding of the sociobiological underpinnings of psychosocial stress. COR's central tenets are relevant to the differential post-deployment experiences of veteran subgroups in that they explain how people seek to obtain, retain, and "protect those things (i.e., resources) they centrally value". Such centrally valued resources include: (1) personal; (2) social; (3) material; (4) and energy resources. Moreover, "resource loss spirals," where losses in one domain (e.g., work schedule reductions) precede greater loses (e.g., bankruptcy) that drive the negative sequela of symptoms (e.g., worry), following initial losses of vital resources, may eventuate in psychological and physical illness (Hobfoll, 1989). Besides structural inequalities in the availability of vital resources between Active and Reserve Component personnel, reservists experience the 
potential added burden of either transitioning back to their former civilian job, or seeking civilian employment within a month post-deployment (Milliken et al., 2007). These stark choices are a result of reservists risking further loss of steady income by accepting unemployment insurance at a fraction of their former active duty pay. For many reservists, who face the dim prospect of supporting families on reduced unemployment benefits, job seeking may take priority over treatment seeking for service-connected medical or psychological issues (e.g., Milliken et al., 2007).

With regard to how PTSD may unfold in the civilian sphere among various veteran subgroups, COR theory would explain a finding, first reported by federal researchers (Shea et al., 2010), of a link between traumatic stress and social functioning during the reintegration phase such that rapid onset of "PTSS" and poor functioning among post9/11 veterans "occurred early" (i.e., within months vs. years) following deployment. Additionally, as predicted by both COR and ALT, differential activation of "effector systems" under varying levels of perceived stressors from resource loss spirals explains how rapid onset of PTSS and poor functioning may be accelerated among reintegrating reservists (e.g., Milliken et al., 2007). Reserve veterans often face unique postdeployment adjustment difficulties and additional life stress from such sources as barriers in accessing high quality healthcare, skyrocketing rates of civilian unemployment (>50\%) coming off deployments (Zavis, 2012) and strained domestic relationships (e.g., McCreary et al., 2014).

By human design, reservists typically cope with lower structural support coming off deployments while also navigating through relatively steeper resource loss spirals 
that contribute to experiencing higher levels of common stressors from balancing multiple work demands and often competing, nonwork family obligations. In addition to these common reintegration stressors for those who continue to serve both their countries and communities in the Reserve Component, there is also the higher incidence of repeated interruptions to their civilian career progress, goals, and overall life satisfaction. Unlike separated and retired veterans, who may more easily focus on their civilian careers, active military reservists know that as post-9/11 military personnel they may be uprooted from their lives and redeployed overseas (Erbes, Kaler, Schult, Polusny, \& Arbisi, 2011). In this sense, Mowrer's (1960) fear conditioning and behavioral learning model in conjunction with Allostatic Load Theory explains how the "recycling phenomena" may be a more destabilizing stressor for reservist veterans. In terms of sources of social capital and support, active reservists are more likely to lack both supportive civilian supervisors and military leaders while depending most on the stability of their civilian careers for their economic survival and their military leaders for their virtual survival. Bound up with these unaddressed subcultural differences among veteran subgroups are the potential effects of anticipation of future deployment.

Anticipatory stress. Ironically, in the current study, active reserve veteran employees were the only group of participants who also coped with the prospect of redeploying (during the collection of their data) to any of the numerous warzones where U.S. troops are currently serving. While almost nothing is known about how up-tempo changes in an active reservists' military job demands (e.g., extended training schedules preceding a possible deployment) impacts current perceptions of stress while also 
juggling civilian employment and family life, COR theory and natural field

observations among primates and applied experiments with humans indicate that the effects of "anticipatory stress" are significant. Randomized control anticipatory stress experiments with workers adjusting to new IT technologies (e.g., Chen, Westman, \& Eden, 2009) and primates undergoing changes in rank and status (Sapolsky, 2007) predict decrements in self-efficacy and increases in symptoms of arousal as a result of releases of cascades of stress hormones (e.g., adrenalin, glucocorticoids) under "anticipatory stress" (Sapolsky et al., 2007) conditions. Likewise, anticipatory stress may also adversely affect active reservists' well-being and health in the absence of sufficient coping resources (e.g., schedule flexibility) unless pre-stressor resources are provided such as stress reduction training for workers before introducing a major technological upgrade (Chen et al., 2009) or greater civilian work schedule control for active military reservists.

\section{Societal relevance of military reintegration}

While it is still unclear whether rapid onset of higher pathological mental health symptoms drive reintegration difficulties or greater reintegration difficulties (e.g., unemployment, sudden separation from war comrades, chaotic family life, low social support, employment discrimination, social rejection, poorly designed jobs) drive higher symptoms, or even whether there are reciprocal effects of symptoms driving reintegration difficulties/stressors that in turn intensify symptoms, what is known is that higher unemployment and workplace discrimination are also linked to "veteran" and "Active Reserve" military status as well as diagnoses of "PTSD" and "depression" (e.g., 
Anderson, 2014; Anderson \& Mitchell, 1992; Savoca \& Rosenheck, 2000). In acknowledgement of the sociological reality of longer and costlier $21^{\text {st }}$ Century wars, requiring the recycling of a more diversified fraction of troops lacking the historical pools of conscripted labor when the burden of service was more equally shared across American society, contemporary reintegration scholars are revising individual and group difference models of the effects of military trauma. That is, reintegration

phenomena may also be conceptualized as a multilevel reality impacting "intrapersonal, interpersonal, community," and cultural systems (Gerhart, Cannetti, and Hobfoll, 2015, p.10). Therefore, like the consequences of the ongoing post-9/11 wars they continue to serve in, the plight of reintegrating post-9/11 veterans may someday be understood as a multifaceted phenomenon with multiple potential predictors and outcomes across distinct veteran subpopulations, thousands of U.S. communities, and hundreds of public and private organizations that directly or indirectly affects most all of us.

\section{Focal variables}

Combat experiences. Surviving war can be stressful for civilians and military personnel, because living within a warzone presents either a direct or indirect threat to one's life. Immediate, direct threats of harm may arrive by way of hand-to-hand combat, close range attacks from lethal weapons (e.g., artillery, aerial bombardment, rocket propelled grenades), being tortured, beaten or assaulted, or contact with toxic or poisonous gases, chemicals or biological agents. Indirect exposure to combat and warzone-related trauma may impact children and more vulnerable members of a society by their being held as prisoners of war, economically and sexually exploited, or having 
their homes, communities, support systems and ways of life destroyed as "collateral damage" of war (Reyes et al., 2008). Accidental injuries or death are also common as are the long-term consequences of unsanitary conditions, starvation, disease and environmental contamination resulting from the ravages of war (Reyes et al., 2008, p.681).

A related but more primitive psychological concept, which is nonetheless still difficult to define, is "trauma". As a source of confusion and controversy in the traumatic stress literature, psychological trauma differs from the medical notion of trauma as "shock and transient or permanent damage caused to the body by severe injury or illness" (Reyes et al., 2008, p. 657). Psychological trauma concerns exposure to catastrophic life events such as "combat, sexual assault, and natural disasters" (e.g., Reyes et al., 2008, p. 657) that produces subjectively experienced distress. However, within the context of war and multiple deployments, the trauma construct is far from a unitary one. Any understanding of trauma is further interwoven with the concept of retraumatization, which can be defined as "exposure to one or more potentially traumatic events" after an initial traumatic exposure that recalls a past psychological trauma and "intensifies the distress related to the prior traumatic experiences" (Reyes et al., 2008, p.586). Contemporary definitions of "trauma" address events that are experienced as "emotionally shocking" or "horrifying", and which convey either "the threat of or actual death or violation of bodily integrity" (e.g., torture, sexual assault, surviving an ambush, awakening during surgery) "or that render the affected person(s) 
helpless to prevent or stop the psychological and physical harm produced" (Reyes et al., 2008, pp. $x$-xii, 587).

Therefore, not every aspect of combat (e.g., "cleaning one's rifle") necessarily entails "trauma"; although, events originally experienced within the milieu of combat can evoke "retraumatization" through traumatic autobiographical memory-mediated recall (i.e., "reexperiencing"). For example, the smell of sulfur burning after striking a match may arouse feelings, memories, and dissociative perceptions of "reexperiencing" a prior mortar attack within a previous combat situation. Experimental evidence (Liberzon \& Abelson, 2016) indicates that altered contextual processing may be related to misperception of semantic and emotional background information that normally allows individuals to grasp situation-informed meaning from environmental cures (i.e., gestalt). That is, previously traumatized individuals may misconstrue not merely stimuli but entire contexts. These "misconstrues" by way of hypervigilance toward decontextualized threatening cues may impair higher cognitive functions related to contextual pattern separation and pattern completion such that separating threat cues from safety cues become confused. An example of which would be after visiting the zoo and then going on safari in Africa one mistakes the roar of a lion for the one most recently observed in captivity. Liberzon and Abelson (2016) proposed that cognitive errors in contextual processing explained the frequently observed recklessness of trauma victims that leads to their propensity for retraumatization.

Adding further complexity to the trauma construct, physical trauma may have profound psychological effects, and nonphysical traumatic stressors (e.g., re- 
experiencing a senseless killing evoking moral injury) may have severe effects on a person's physiology (Reyes, 2008, p. 657). Combat exposure has been operationalized as "the length of time in military service or in a combat zone" and combat experiences as individually perceived combat situations (e.g., witnessing dead bodies, surviving roadside bombs) with higher rates of exposure to combat situations and combat experiences of perceived danger (e.g., hand-to-hand combat) or distressing events (e.g., witnessing the death of friends, killing/torturing of innocents) being generally associated with higher levels of posttraumatic stress symptoms (e.g., Reyes et al., 2008, p.586). As researchers continue to explore how experiences of trauma may differ, both in kind as well as between individuals and groups on measures of health and well-being, it is becoming increasingly clear that any conceptualization of stressors as reported by surviving combatants are by nature complicated and limited by the interactions of both the original and host cultures as well as the developmental stages of those experiencing them. Dr. Rachel Yehuda, Ph.D., a professor of psychiatry and director of traumaticstress studies at Mount Sinai Hospital in New York, has offered several paradoxical insights on the effects of combat trauma on the developing human mind:

For most people in combat, their experiences range from the best to the worst of times. It's the most important thing someone has ever done-especially since these people are so young when they go in - and it's probably the first time they're every free, completely of their societal constraints. They're going to miss being entrenched in this very important and defining world (Junger, 2015).

In total, the range and variety of psychological and physical stressors that combatants may experience before, during, and after war is vast. Researchers are just 
beginning to disentangle how different levels and kinds of trauma and combinations of combat exposures and combat experiences may alter the health, wellbeing and quality of life as well as interpersonal, occupational, and social functioning of people surviving life in a warzone (Ford, in Reyes et al., 2008, p. 682). Though any scientific study of military reintegration involves the disaggregation of the retrospective traumatic memories that may be fraught with hindsight biases, the present thesis study is among the first work integration studies this author is aware of to explore the multidimensional effects of the experiences of combat.

Veteran Employee Subgroups. Prior studies of military workers suggest that the process of "military stress" is similar to other high-demand occupations, with increased rates of burnout, that may "spill over into other areas of post military employment," especially if functioning is impaired (Hobfoll et al., 2012). In the present study, the idea of identifying subgroups of working veterans and linking them within the larger organizational context of their most recent "military affiliation" is motivated, in part, by the theory of "allostatic states" where significant psychosocial stressors cause elevated activation of effector systems underlying the physiological hypothalamic-pituitaryadrenal axis (i.e., HPA-axis) stress response. A common objection to perceived stress models is the classic "mind-body" problem of explaining how, precisely, our "minds" and mental thoughts influence our physical bodies. In short, the process of allostasis connects the brain and coordinates thought with "body-wide changes often including changes in behavior" through such mechanisms as the neuroendocrine system (Sapolsky, 2004, p.9). Over time, shifting perceptions of competing job demands and 
work pressures from varying occupational sources would be expected to result in fluctuating alterations of effector system set points among "Active Reserve Veteran Employees" relative to other groups of U.S. workers with similarly matched military backgrounds and combat experiences (e.g., "Separated Active or Reserve Component veteran employees). Like serendipitous field observations with baboon troupes experiencing the unexpected decimation of aggressive alpha males who suddenly died en mass after raiding spoiled meat from a dump (Sapolsky, 1983), in SERVe a naturalistic experimental condition occurred whereby active reservist veteran employees were the only veteran participant subgroup to hold contemporaneous employment in both the civilian and military contexts. Therefore, active veteran reservist employees (ARVEs) may differ from their separated veteran employee counterparts on perceived levels of iso-strain, sleep quality, and PTSS.

Given this naturalist experimental condition the allostatic load perspective informed a sharper occupational health focus on grouping SERVe combat veteran employees according to their self-identified membership among either: (1) holders of a second paid military occupational specialty (MOS) while also holding at least one civilian job of twenty or more hours per week (i.e., ARVEs); or (2) holders of only a civilian job of at least twenty or more hours after separating from the military, permanently (i.e., Separated Active Component or Separated Reserve Component). In addition, empirical support for the premise that veteran employee subgroup (VES) may moderate work-family phenomena is provided by a recent military reintegration study (McCreary et al., 2014) that found significant interactions between single and married 
Canadian armed forces with and without dependents but only on family negative reintegration scores. These results suggest that military membership within the family domain may alter perceptions of non-work reintegration experiences while leaving open the question of whether the civilian work domain differentially enhances or buffers perceptions of posttraumatic stress following combat by veteran employee subgroup.

\section{Organizational variables}

Classic work stress studies dating to Karasek and Theorell (1990) focused on the effects of job demands and job control (i.e., decision latitude, skill discretion) as well as the proposed interaction between them (job demands $x$ decision latitude) among civilian work samples being related to job strain. In the present study job demands and job control were expected to be differentially related to PTSD. However, since social support has been identified as vital protective factor for posttraumatic stress (e.g., Heinrichs, 2003; Olff, 2013), the more theoretically complex and understudied composite variable "iso-strain", which has been inconsistently operationalized by scholars (e.g., de Mello Alves, Hokerberg, \& Faerstein, 2013; Kuper \& Marmot, 2003; Mather et al., 2015), was proposed as a moderator of combat experiences on PTSS. Encompassing the cumulative relative effects of demands, control, and support at work, theory and prior research (e.g., Karasek, 1979; Johnson, Hall, \& Theorell, 1989; Mather et al., 2015; Schaufeli \& Taris, 2014) proposes that iso-strain is a multidimensional construct composed of job demands, job control, and workplace social support.

Job demands. Karasek (1979) posited that job demands and job control are the two most relevant factors in a worker's experiences of job stress. Job demands include the 
"workload" and "intellectual requirements" of employment (Karasek \& Theorell, 1990). Specifically, workload concerns the pace of work, speed of work, hardness of work, and time pressure as well as the concentration and attention required to meet the demands of a job within a work environment (Karasek, 1979; Karasek, Brisson, Kawakami, Houtman, Bongers, Amick, 1998). As a potential source of strain, work overload refers to a worker's perception of when the "environmental situation poses demands which exceed" the worker's capacity to meet them (Karasek, 1979, p.287). Further, Karasek proposed that the interaction of "high work demands" and "low control" characterizes "high-strain" jobs (e.g., bus driver, infantrymen) that lead to health and psychological problems such as cardiovascular disease, fatigue, psychosomatic complaints and depressive symptoms (Karasek, 1979). However, mixed and limited evidence (e.g., Luchman \& González-Morales, 2013; Wall, Jackson, Mullarkey, \& Parker, 1996) supports the Demand-Control Model's (DCM) prediction that job control alone attenuates high job demands on strain. Such early job stress theories as Karasek's DCM are vague on how different kinds of social support, resources, and specific job strain conditions (e.g., "iso-strain" environments) may influence the effects of stressors on strains.

Job control and decision latitude. Job control concerns the degree of autonomy and discretion in the application of skills that organizations allow their workers to apply to meet the demands of their employment situation (Karasek et al., 1990). Job control may also be operationalized as "decision latitude," which is comprised of "two components: skill discretion and decision authority" (Van der Doef \& Maes, 1999, p.88). Whereas, 
decision latitude and job control refer to the extent of perceived control one possesses over decisions concerning their job and how job demands are completed, Karasek (1979) defined job strain as the interaction between high job demands and low control or decision latitude. To date, empirical results on the theoretical job strain interaction of high demands and low control (i.e., 'decision latitude') have been inconclusive (e.g., Landy \& Conte, 2013).

Social Support. Among the most potent social and personal resources to counter the negative effects of psychosocial stress, social support ranks among the most vital and emotionally satisfying coping strategies that people possess to ease life's challenges and help with overcoming adversity (e.g., Taylor, 2010, p. 707). Among 14 analyzed risk factors for PTSD (e.g., trauma severity, gender) social support had the largest effect size (Brewin et al., 2000). Psychologically, Taylor (2010) defined social support as the degree to which one perceives, feels or experiences that they are loved and cared for by others, esteemed and valued, and part of a larger social network of mutual obligations and information-sharing (e.g., Friedman, 2011, p.189). Supportive social networks may exist among parents, spouses, coworkers, lovers, relatives, friends and one's larger community as often found in social clubs, churches, professional guilds and organizations and even faithful pets (e.g., Allen, 2003; Rietschlin, 1998). Numerous studies reveal that being embedded in a social network (i.e., social integration) - with sufficient instrumental, informational, appraisal, and emotional support with or without high stress - are all related to health and well-being (e.g., Cassel, 1976; Taylor, 2010; Warr, 2007). 
Coworker Support (CWS) and Supervisor Support (SS) are operationalized as social support exchanged by co-workers and supervisors at work. According to Langford, Bowsher, Maloney and Lillis (1997), CWS may be given in four ways: (1) emotional support such as caring, empathy, and trust; (2) instrumental support (providing tangible goods or aid); (3) informal support (help in problem solving); (4) appraisal support (affirming or communicating self-evaluation). Psychosocial resources such as CWS may affect similar physiological mechanisms that produce healthprotective effects (Taylor, 2010). As predicted by Leader-Member Exchange (LMX) theory, supervisors and the quality of LMX relationships between subordinates and superiors are a potential source of employee social support (Chen, Wang, Chang, \& Hu, 2008). Further, because of their institutional role as linchpins between upper management and lower-level employees, organizations typically empower supervisors to implement work-family policies, which may deter employees from taking advantage of them out of fear of retaliation (Glass \& Fujimoto, 1995; Raabe, 1990). Alternatively, organizations and supervisors who support their employees' nonwork demands (e.g., family obligations) may still not have high utilization rates of such family friendly policies, because of the variability of how organizations implement employee workfamily policies and how supervisors may vary in their support for employees. Therefore, informal supervisor support in facilitating employee balance of their work and family demands may have a larger effect on employee health and well-being than formal organizational supports (e.g., Allen, 2001; Behson, 2005; Kossek \& Nichol, 2006). 
For example, family supportive supervisors such as scheduling flexibility for active reserve veteran employees (ARVEs) may provide periodic stress relief. That is, an active reservist employee's ongoing military service obligations may pose periodic work scheduling conflicts and prolonged leaves of absences from their civilian jobs due to redeployment. Supervisor support has been shown to act as a beneficial resource that may buffer the deleterious impact of work and family stressors (e.g., O'Driscoll, Poelmans, Spector, Kalliath, Allen, Cooper, \& Sanchez, 2003; Thomas \& Ganster, 1995). Also, supervisor support is linked to reduced work-family conflict (e.g., Anderson, Coffey, \& Byerly, 2002; Frone, Yardley, \& Markel, 1997) and objective and subjective sleep quality and quantity (Crain, Hammer, Bodner, Kossek, Moen, Lilienthal, \& Buxton, 2014) and reduced experiences of workplace distress (e.g., Frone, Russell \& Cooper, 1997), reduced turnover intentions (e.g., O’Neill, Harrison, Cleveland, Almeida, Stawski, \& Crouter, 2009; Thompson, Beauvais, Lyness, 1999; Thompson \& Prottas, 2005), lowered absenteeism (Goff, Mount, \& Jamison, 1990), and higher organizational commitment (O'Neill et al., 2009). In the present study, the combined influences of supervisor support and coworker support (i.e., Workplace Social Support) were expected to be the main sources of civilian organizational support for veteran employees. As such, the degree to which the supervisors of veteran employees are supportive or unsupportive of their work and nonwork demands (e.g., time off for military duties, family obligations, medical appointments) was expected to contribute to ARVEs' perceptions of the manageability of balancing their concurrent 
civilian work demands, military duties, and family obligations and thereby affect their perceived symptoms of posttraumatic stress.

Linking Social Support with PTSD through sociobiology. A well-documented physiological process first identified in the 1950s that may produce the observed effect of social support buffering symptoms of strain under high stressors is the role of oxytocin within the mammalian neuroendocrine system. In 1954, du Vigneaud and colleagues experimentally isolated the nonapeptide oxytocin for which he won the Nobel Prize (du Vigneaud, Ressler, Swan, Roberts, \& Katsoyannis, 1954). Produced by the hypothalamus and stored and secreted by the posterior pituitary gland, oxytocin primarily acts as a stress-reducing neuromodulator. Neurobiological research reveals oxytocin's role in: (1) promoting affiliative activities in response to stress; (2) secretion in response to stress; and (3) dampening sympathetic nervous system (SNS) and hypothalamus-pituitary adrenal (HPA) axis responses to stress (e.g., Taylor, Dickerson, \& Klien, 2002; Uvnas-Moberg \& Petersson, 2005). Both the SNS and HPA are welldocumented physiological mechanisms underlying arousal symptoms and other requisite neuroendocrinological reactions involved in PTSD (e.g., Yehuda, Hoge, McFarlane, Vermetten, Lanius, Nievergelt et al., 2015) as well as an established biological route for the theoretical influence of the JD-R's health impairment process (Demerouti \& Bakker, 2011). In sum, the physiological function, action and psychological effects of the observed social support x oxytocin interaction as a proposed biopsychosocial process with growing empirical support explains how a neuroendocrinological mediator (i.e., oxytocin) of psychosocial stressors may be 
affected by relational factors such as social support (e.g., Heinrichs, Baumgartner, Kirschbaum, \& Ehlert, 2003; Holahan, Moos, Holahan, \& Brennan, 1997) to reduce symptoms associated with PTSD (e.g., Olff, Koch, Nawjin, Frijiling, Van Zuiden, \& Veltman, 2014). Thus, though unmeasured in the proposed study, cognitive-behavioral and sociobiological (i.e., biopsychosocial) theories involving how patterns of social interactions effect human cognition, neuroendocrinology, affect, and behavior offer a biopsychosocial process relevant to theorizing how work situations (e.g., iso-strain) and individual differences (e.g., insomnia) may moderate the theoretical pathway between experiences of combat stressors and PTSS.

Buffering hypothesis. The "Buffering Hypothesis" (Cohen \& Willis, 1985) focuses on the relations among stress levels, coping, social support and adjustment. However, inconsistent results from empirical tests of the DCM's central tenet of the theoretical buffering effect of high levels of job control (e.g., Dwyer \& Ganster, 1991; Sargent \& Terry, 1998; Spector, 1987) have been attributed by organizational scholars to conceptual and methodological flaws in prior research designs (Jimmieson \& Terry, 1999; Wall, Jackson, Mullarkey, \& Parker, 1996). For instance, models based on social exchange theory have found three-way interactions of social support such that the buffering effect of support on the main effect of work hours on employee health and well-being varied as a function of the pattern of three-way exchanges (e.g., underreciprocating, over-reciprocating, reciprocal) between an employee and sources of close support (Nahum-Shani \& Bamberger, 2011). 
Such limitations of the DCM and experimental research into the buffering hypothesis led to an extension of the DCM: The Job Demand-Control-Support (JDC-S) model (e.g., Van der Doef et al., 1999). According to the JDC-S, job demands, job control, and "worksite social integration" are relevant factors in "the development of health problems" (e.g., Van der Doef et al., 1999, p. 89). Under certain conditions, however, evidence (e.g., Cohen \& Willis, 1985) suggests that social support that matches the coping need of the recipient can attenuate the effects of stressors (e.g., job demands) on strain reactions (e.g., PTSS). Where the "buffering hypothesis" predicts that greater levels of the moderator (i.e., healthy sleep) reduces the effect of the predictor (i.e., combat experiences) on the dependent variable (i.e., PTSS), the enhancing hypothesis, by contrast, predicts that greater levels of the moderator (i.e., isostrain) will increase the effect of the predictor on the dependent variable.

Iso-strain. As I-O scholars continue to apply various schemes to operationalize isostrain (ISO), in the present study ISO was conceptualized as a composite construct measured by the relative effects of job demands, job control plus schedule control, and social support at work from coworkers and supervisors. Motivation to test the effect of iso-strain over job demands, job control, and social support independently was a result of two considerations: (1) efficiency of measurement design and modeling simplification, which captures all three principal components (i.e., demands, control, and support) in a single measure; and (2) deficits in earlier job stress theories such as the Demand-Control Model (DCM), which were vague on how different forms of social support, resources, and specific job strain conditions such as "iso-strain environments" 
may influence the main effect of job demands and other work stressors on strain outcomes. Employee health and wellness outcomes linked to iso-strain work conditions are serious and include higher risk for coronary heart disease among young workers, whom may be resistant to modification by workplace social support (e.g., Kuper \& Marmot, 2003), and sick leave due to mental illness (Mather et al, 2015).

\section{Individual differences variable}

Insomnia/sleep quality. Prevalence studies of insomnia rank it as the number one sleep disorder with up to a third of the general population reporting sleep difficulties and about 10\% seeking medical treatment for their chronic symptoms (Basta, Chrousos, Vela-Bueno, Vgontzas, 2007). Medically, insomnia is defined as complaints over "difficulty falling asleep or staying asleep", including "early awakening" and "interrupted or non-restorative sleep" that "results in daytime consequences" (e.g., Basta et al., 2007; Rissling \& Ancoli-Israel, 2009, p.79). Diagnosis of insomnia requires significant distress and/or impairment in daytime functioning as well as exclusion of other mental health problems (e.g., depression), sleep disorders (e.g., apnea), substance abuse or other medical conditions (APA, 2013). Insomnia may be underreported with as many as $60 \%$ of those afflicted neither seeking treatment nor discussing their sleep difficulties with a physician (Basta et al., 2007).

Insomnia and sleep problems are much more common among military personnel than among the general public. For example, a recent cross-sectional analysis of Active Duty $(N=761)$ post-9/11 military, referred for polysomnograms, revealed that more than 40\% (41.8\%) slept under 5 hours per night (compared with 7.8\% of US civilians) with 
diagnosis of insomnia associated with twice the odds of comorbidity for PTSD (Mysliwiec, McGraw, Piece, Smith, Tapp, \& Roth, 2013). Recently, a consensus of sleep experts (Watson, Badr, Belenky, Bliwise, Buxton, Buysse et al., 2015) advised that adults should obtain seven or more hours of sleep, regularly per night, to avoid risking their health. Poor sleep among workers is also linked with occupational accidents (Akerstedt, Fredlund, Gillberg, \& Jansson, 2002a), reduced work productivity (Hamilton, Gallagher, Preacher, Stevens, Nelson, Karlson et al., 2007), absenteeism (Spector \& Jex, 1998), and turnover (Schimizu, Horiguchi, Obata, \& Nagata, 2005). In a national survey of U.S. adults $(N=3,643)$ insomnia was negatively related to both psychological and subjective well-being (Hamilton et al., 2007). Briefly, psychological well-being is composed of autonomy, environmental mastery, personal growth, positive relations, purpose in life, and self-acceptance. While subjective well-being is composed of positive and negative affect and life satisfaction (Hamilton et al., 2007).

Insomnia is often an antecedent to depression and anxiety and sleep disturbances (van Liempt, 2012) and is a core symptom of hyperarousal associated with PTSD (Mysliwiec et al., 2013). As many as 90\% of those exposed to trauma and diagnosed with PTSD report "disturbing dreams", resembling details of the actual traumatic event, and "persistence" of disrupted sleep and nightmares "1 month posttrauma" increases risk of PTSD (Germain \& Zadra, 2009, p. 316). As symptoms of disturbed sleep such as nightmares and insomnia are hallmarks of PTSD (e.g., APA, 2013; Germain et al., 2009) - with nightmares typically indicating "reexperiencing" and "insomnia" typically indicating "hyperarousal" (e.g., Basta et al., 2007) — improving both the quality and 
quantity of the sleep of veterans would have important consequences for organizations and employees. In addition, insomnia is a well-documented risk factor and likely maintenance mechanism for PTSD (e.g., Germain, 2013).

Insomnia/PTSD overlap. The overlap of PTSD and insomnia extends to the biological and psychosocial levels, too. In a review of the clinical insomnia literature, Basta and colleagues asserted that the accumulated scientific and clinical observations supported a model of insomnia being "a state of 24-h hyperarousal" rather than solely attributable to "sleep loss" (Basta et al., 2007, p.10). Paradoxically, these authors concluded that compared with normal sleepers, insomniacs were "not sleepier during the day" but rather had "elevated brain metabolism during sleep" that "decreased during wakefulness" but with "similar cognitive function and performance" as good sleepers (Basta et al., 2007, p.10). The authors interpreted these counterintuitive results as being explained by the 24-hour over-activation of the HPA-axis in insomniacs along with “hypersecretion and/or circadian alteration of cytokines' secretion" (Basta et al., 2007, p.10). Similarly, theoretical models of hyperarousal subtypes of PTSD (e.g., Weston, 2014) explain the importance of the hyperarousal cluster of symptoms in that peritraumatic responses (i.e., reactions around the time of the trauma) co-occur with hypersecretions of stress hormones and physiological arousal leading to amygdala processing of hyperarousal. In this model, hyperarousal is the proposed antecedent symptom and driver of autobiographic memories as well as other core PTSD symptoms (i.e., reexperiencing, avoidance). In addition, insomnia is related to depression and "anxious-ruminative personality traits, stressful events," age, and "genetic-diathesis of 
CNS hyperarousal" (Basta et., 2007, p.10). Taken together, improving the sleep of workers and veteran employees and reducing risk of onset of insomnia may offer a potential buffer of the effects of combat experiences and workplace stressors on posttraumatic stress symptoms.

Linking Sleep quality with PTSS. Considerable evidence suggests that sleep is both an individual resource and indicator of general health and well-being (e.g., Hamilton et al., 2007). Specifically, sleep latency (i.e., onset of sleep) and non-restorative sleep (i.e., disturbed sleep) are both components of insomnia (e.g., Basta et al., 2007), and insomnia and disturbed sleep (e.g., "nightmares") among war veterans are wellestablished risk factors for PTSD (e.g., Germain, 2013; Germain, Buysse, Shear, Fayyad, \& Austin, 2004; Germain \& Zadra, 2009; Insana, Hall, Buysse, \& Germain, 2013). Taken together, it was expected that global sleep quality would be related to symptoms of posttraumatic stress. In the manner outlined by established sleep researchers (e.g., Buysse, Reynolds, Monk, Berman, \& Kupfer, 1989) the proposed study operationalized sleep quantity as the amount of time a participant spent in a retrospectively self-reported sleeping state. Whereas, sleep quality indicated the selfreported degree of difficulty of falling asleep or staying asleep and the number of nocturnal awakenings.

Recently, in their population-based study ( $N=958$ ), Morin, Belleville, Belanger and Ivers (2011) reported a high positive correlation $(r=0.80, p<0.05)$ between an index of insomnia severity (ISI) and the Pittsburgh Sleep Quality Index (PSQI) global score (Buysse et al., 1989). Again, though related to "sleep quality" medically insomnia refers 
to either (1) "difficulty maintaining or initiating sleep," or (2) "nonrestorative sleep for at least 3 weeks for 3 months despite adequate opportunities" (APA, 2013, pp.362363). These two facets of clinical insomnia (i.e., difficulty sleeping and nonrestorative sleep) are also two dimensions of Buysse and colleagues' (1989) multidimensional PSQI. In SERVe, the PSQI measured the quality of veteran sleep. Prior social survey and clinical research has demonstrated that the PSQI possess excellent psychometric properties (e.g., Beck, Schwartz, Towsley, Dudley, \& Barsevick, 2004). To be clear, though theories of insomnia and its potential impact on PTSD informed the present thesis study, scientifically self-reported sleep quality is only a proxy for clinical insomnia, which requires a diagnosis by a licensed medical professional (APA, 2013, pp.362-368) typically after obtaining results from an overnight sleep study (i.e., polysomnography).

\section{Outcome variable}

Posttraumatic Stress Disorder (PTSD). The inclusion of PTSD as a diagnosable medical condition 37 years ago marked "a paradigm shift in the conceptualization of post-trauma illness" (Jones \& Wessely, 2007, p.164). Recognition of PTSD by the medical establishment designated a change from a cultural focus on group influences to greater clinical attention on individual experiences and "the characteristics of the event itself." Some researchers have observed that the medicalization of PTSD transferred primary causation away from the person to the "terrifying experience" (Jones et al., 2007, p.165). Presently, the clinical construct of PTSD is an evolving one with its most recent reconfiguration by the psychiatric establishment as now including a fourth 
additional cluster of symptoms (i.e., negative alterations in cognitions and mood).

However, there is a notable "absence of scientific understanding" of the pathophysiology of PTSD (Liberzon \& Abelson, 2016). Consequently, expert consensus "as reflected in DSM criteria" is still considered "the gold standard" (Liberzon \& Abelson, 2016). According to the prevailing clinical model (e.g., DSM-V, 2013), PTSD may occur following traumatic exposure(s) outside the normal range of human experiences (e.g., the unusual or traumatic death of a close relative, threatened or actual harm, injury or serious accidents, combat). Since no one is born with PTSD, and those exposed to similar traumas do not similarly acquire PTSD, logic dictates people should vary in their susceptibility to PTSD.

Evolution of theories of PTSD. Until as recently as 2000, a twenty-year consensus of mental health experts had proposed that such stressors as combat exposures (i.e., "Criterion A", APA, 2000) were causally related to symptom clusters of PTSD (Dohrenwend, Yager, Wall, \& Adams, 2013). Other prominent conceptualizations of PTSD have ranged from information processing, and anxious apprehension models to emotional processing and dual representation models, and more recently social cognitive and cognitive appraisal and coping theories (Brewin \& Holmes, 2003). However, research based on the studies of Vietnam-era twins concordant and discordant for combat exposures in Southeast Asia and non-shared environmental factors revealed that the path from combat stressors to PTSD was largely indirect and that multiple pathways to acquiring the disorder are most likely (e.g., Sapolsky, 2002; Wolf et al., 2014). For example, among combat exposed twins, the effects of genetic and non- 
shared environmental factors on PTSD appeared stronger at higher levels of combat exposure (Wolf et al., 2014). Whereas, in a retrospective study of neurophysiological vulnerability, a smaller hippocampi predicted later onset of PTSD even when predicting from the non-combat exposed twin to the combat-exposed twin (e.g., Gilbertson, Shenton, Ciszewski, Kasai, Lasko, Orr, \& Pitman, 2002; Sapolsky, 2002).

According to a recent genetic analysis of veterans and adults, as much as $46 \%$ of the variance in PTSD may be explained by genetic factors, leaving the remainder "attributable to the non-shared environment" (Wolf et al., 2014, p.1499). Though Wolf and colleagues (2014) concluded that the Diathesis-Stress Theory (DST) best explained the finding of three predictors (i.e., combat exposure, prewar vulnerability, and harming civilians or prisoners) accounted for 97\% of the variance in PTSD cases, RAND researchers (Tanielian \& Jaycox, 2008, p. 122) have critiqued the DST model for being mostly useful at predicting individual risk for disease under certain environmental exposures or after experiencing psychosocial stressors (e.g., iso-strain) while failing to explain "how mental disorders" influence "further difficulties throughout the life course". Thus, as shown in Figure 2., in order to meet clinical diagnostic significance, one or more precipitating "Criterion A" stressors are required that lead to such core symptoms as: (1) persistent reexperiencing/intrusion (e.g., "flashbacks", nightmares); (2) avoidance of traumatic cues and reminders (e.g., public firework displays, stressful work situations); (3) alterations in arousal/reactivity (e.g., anger, insomnia); and (4) negative alterations in cognitions and/or mood (e.g., guilty 
self-blame) that persist for at least one month (APA, 2013; Groer, Kane, Williams, \& Duffy, 2015).

Occupational functioning and PTSD. Though research is still vague on how the civilian work environment may shape PTSS and/or PTSD, evidence from two longitudinal studies may provide researchers with some preliminary insights. Results from a 20-year longitudinal study of Israeli combat veterans $(N=214)$ showed that PTSD symptoms can vary over time and between clinical and nonclinical groups and predicted social functioning two decades later (Solomon, Horesh, \& Ein-Dor, 2009). For example, the clusters of intrusion, avoidance and hyperarousal were interrelated at multiple time points (Solomon et al., 2009). While clinical and nonclinical groups differed in intrusion and avoidance over time, on hyperarousal both clinical and nonclinical reported similar symptom levels (Solomon et al., 2009). Interestingly, for both groups avoidance was the most stable symptom cluster over time while hyperarousal was the most stable only within the clinical group such that for the nonclinical group hyperarousal at Time 1(1983) predicted avoidance and intrusion only at Time 2 (1984) (Solomon et al., 2009); whereas, for the clinical group the effect of hyperarousal at Time 1 lasted decades longer (Solomon et al., 2009, p. 842). This fits with Shea et al.'s (2010) more recent finding that “avoidance/numbing” and "hyperarousal" were more predictive of occupational functioning than "reexperiencing". More broadly, results from Vietnam-era twin studies revealed direct and indirect pathways to PTSD that are also supported by a weighted reanalysis of a subsample $(N=1,265)$ from the NVVRS, which found that less than a third $(32 \%)$ of 
Vietnam veterans who had experienced "Criterion A" stressors developed PTSD

(Dohrenwend et al., 2013). In the future, Dohrenwend and colleagues (2013)

recommended that researchers investigate pre-stressor genetic vulnerabilities as well as how stressful situations (e.g., iso-strain) may exacerbate symptoms or evoke "recurrence" of PTSD.

\section{Background, individual, and military factors related to PTSD}

In their final report to the United States Congress the authors of the NVVRS (1990) revealed an exhaustive analysis of statistical procedures and tests to determine whether one's experiences within the theatre of war or "predisposing selection factors" and other demographic variables were most related to the observed effect of warzone experiences and combat on posttraumatic stress disorder (Kulka et al., 1988/1990). To their credit, Kulka and colleagues (1990) clearly demonstrated that socioeconomic forces largely determined who served in the military and who went to Vietnam and that such selection factors played a large role in a war veterans' postdeployment mental health (Kulka et al., 1990, p.81). Overall, however, adjustment for such predisposing factors as childhood and family background (e.g., socioeconomic status), pre-enlistment general health (e.g., physical and mental), and military experiences (e.g., non-combat duty vs. warzone experiences) decreased the prevalence of PTSD between theater veterans and comparison groups (Kulka et al., 1990, p.81). However, in the final analysis these adjustments did not eliminate the effect of warzone stress as the major contributor to post-deployment psychological distress (Kulka, 1990). Informed by Kulka and other military researchers (e.g., Bonanno et al., 2012; Bliese, McGurk, 
McBride, \& Castro, 2011), and utilizing a post-hoc correlation analyses of ten candidate factors, five control variables were selected for this study's main statistical analyses.

Socioeconomic status. The influence of socioeconomic status (SES) on health, psychological well-being, and longevity is a consistent finding in stress and health research with both military and civilian populations. More specifically, the "Socioeconomic Gradient" is a recent formulation of the predictive association between social class and health. For example, the U.S. Panel Study of Income Dynamics sampled 8,500 men and women over twenty years (1972-1991). Risk of dying was associated with average household income. Results reveled that relative risk of death in each income group increased relative to the wealthiest group (>\$70k). Typically, SES is measured by indicators of education, income, occupation, or a composite of these dimensions. Although in epidemiological studies education is the most commonly used measure of SES, only recently have researchers conducted empirical analyses quantifying the relative impact of each separate dimension of SES on risk factors for disease. Using data on a large sample $(N=2,380)$ of mostly white, non-Hispanic participants (85\%), Winkleby, Jatulis, Frank, and Fortmann (1992) tested the independent contribution of education, income, and occupation on predictors (i.e., cigarette smoking, systolic and diastolic blood pressure, and total and high-density lipoprotein cholesterol) of cardiovascular disease (CVD). Among indicators of SES results showed that education was the largest independent contributor associated with risk factors for CVD. In this study, higher risk for CVD was associated with lower levels of education. Subsequent studies with people and primates have consistently 
supported the phenomena known as "The Socioeconomic Gradient," revealing the influence of SES in determining health, longevity, and wellness (Sapolsky, 2004). In the present study, SES was comprised of three indicators: military rank; educational attainment; and a global score of perceptions of financial strain (Sinclair \& Cheung, 2016).

Time since last deployment. Military studies consistently show that the length of time following deployment is related to physical and mental health symptoms, including posttraumatic stress (e.g., MacGregor, Han \& Galarneau, 2011). What is less clear is whether the direction of this association is necessarily positive or negative. For some veterans, the amount of time since last deployment may occur within the context of reintegration and post-reintegration, and may be negatively associated with posttraumatic stress symptoms, especially among separated veteran service members who have permanently returned to civilian life. Logically, however, it would be expected that this association may not always be consistent as "time since last deployment" may also occur within the context of continued military service such as among active duty service members and active veteran reservists with at least one deployment. In the context of continued military service, especially among troops performing high-tempo military occupational specialties (e.g., infantry, aerial combat), "time since last deployment" may actually be perceived as "dwell time", or the recovery time a military member experiences before training for and mobilizing into their next deployment (Yosick, Bates, Moore, Phillips, \& Davison, 2012). 
Risk of Homelessness. The earliest records of homelessness among U.S. veterans date to the Reconstruction (1865-1877) following the Civil War (Tsai \& Rosenheck, 2015). It would take another 100 years, however, for the reality of veteran homeless to draw national attention as a public health issue (Tsai \& Rosenheck, 2015). In their systematic review of the literature on risk factors for homelessness among U.S. veterans, Tsai and Rosenheck (2015) included the seven most rigorous studies published since 1994. The authors found that the most consistent risk factors for veteran homelessness were substance abuse and mental illness with substance abuse contributing the more robust effect whereas PTSD showed no difference in magnitude over other mental disorders. Other relevant predictors were socioeconomic status, military pay grade, and unemployment with compensation for VA service-connected disability demonstrating a protective factor against homelessness.

Conscientiousness and neuroticism. Early occupational health methodologists once warned that failing to measure third variables such as "stable personality traits" like "negative affectivity" had confounded "stressor-strain correlations" in prior research (Zapf, Dornman, \& Frese, 1996, p. 153). Further, as the preponderance of evidence from two generations of military studies shows that less than half of combat exposed veterans went on to acquire PTSD (e.g., Brewin et al., 2000), an understanding of which person-level factors differentiated those troops most at risk of PTSD would help inform predictions on potential individual differences that may covary with PTSS. Therefore, controlling for personality traits (e.g., Jakšić, Brajković, Ivezić, Topić, \& Jakovljević, 2012) would help ensure that any detected effects of the hypothesized associations with 
PTSS were not due to an overrepresentation of relatively stable individual factors such as the personalities of research participants. In addition, occupational health researchers who have studied individual differences in connection with the stress process have focused on isolating individual factors that dispose people to engage in adaptive and maladaptive coping with work stressors (e.g., Nelson \& Simmons, 2011). Individuallevel variables that have been previously investigated include Type A/B behaviors, optimism, locus of control, negative affect, secure attachment and hardiness (Nelson \& Simmons, 2011). Likewise, over the last several decades, military researchers have probed whether several individual factors (e.g., optimism, pessimism, education, hardiness) are protective against PTSD. Among this list, trait/dispositional hardiness defined as commitment (i.e., high engagement with life while imbruing persons and situations with meaning), challenge (i.e., viewing threats as opportunities for growth combined with an ability to withstand ambiguity), and control (i.e., one's belief in their capacity to exert influence over their life) - have been conceptualized as working in concert to produce psychological and physical health (Nelson \& Simmons, 2011, p.64).

In their exhaustive studies of the influence of personality, military researchers have positioned dispositional 'hardiness' as an antecedent to resilience (i.e., healthy, adaptive outcomes) following adversity, trauma, or hardship. For example, Kobasa and Maddi (1979/1982) first proposed the psychological construct of hardiness as containing self-reflective dimensions on one's place in the world simultaneously expressed through their actions, thoughts and feelings (Kobasa, 1979; Kobasa, Maddi, \& Kahn, 1982), which are conceptualized as representing the independent dimensions 
of "commitment, challenge, and control" (Nelson \& Simmons, 2011, p.64). Ideally, people high in hardiness and optimism are expected to engage in "transformational coping" by redefining a stressor in a way that attenuates and reframes its menacing qualities as "growth opportunities". By contrast, those low in hardiness and higher in neuroticism would be expected to be more susceptible to the effects of combat and trauma and negative affect. According to Maddi (1999) hardiness is also related to social support seeking and engaging in health-promoting behaviors as was found in a retrospective analysis of supervisors at Illinois Bell Telephone where managers high in hardiness experienced fewer mental and physical illness symptoms (Maddi, 1999).

Recent studies of military populations found that hardiness is related to reduced levels of PTSD in addition to other psychiatric symptoms (King, King, Fairbank, Keane, \& Adams, 1998), and increased perceptions of meaningful work (Bartone, 1999). More recently, however, in a validity study of hardiness among a post-9/11 military sample results were mixed. Minnesota VA researchers (Erbes, Arbisi, Kehle, Ferrier-Auerbach, Barry, \& Polusny, 2011) tested the construct and predictive validity of Bartone's (1995) “Short Hardiness Scale” (SHS) on a large, demographically homogenous sample of Army National Guard soldiers ( $N=981)$. Among combat exposed Army National Guard soldiers factor analysis revealed that two personality traits (i.e., conscientiousness, neuroticism) were associated with hardiness and pessimism and positive and negative affect. Surprisingly, in contrast to prior military studies, hardiness did not incrementally predict symptoms of PTSD (Erbes et al., 2011). Therefore, following Erbes et al.'s (2011) finding of hardiness being a related but 
distinct construct from positive emotionality (PEM) and negative emotionality (NEM), and that neuroticism and conscientiousness were, respectively, positively and negatively related to PTSD, these two personality traits were selected a priori as control variables in the present study.

\section{Linking combat, work characteristics, and individual factors to PTSS. Hobfoll} and colleagues (2012) recent exploration of the link between combat and PTSS among returning Air Force veterans found that "exposure to trauma" predicted increased PTSS symptoms and greater loss of resources (e.g., personal and social relationships, finances, career advancement, and pride in military service). Further, in the same study, "exposure to trauma" fully mediated the effects of "deployment to a combat theatre" on both baseline $\left(T_{1}\right)$ PTSS symptoms and at follow-up fourteen months later $\left(T_{2}\right)$ as well as resource loss. Interestingly, however, deployment to a combat theatre (versus deployment to a non-combat theatre) predicted an increased level of exposure to trauma but not elevated PTSS symptoms.

Hobfoll and colleagues' (2012) findings suggest that specific combat experiences (CES), rather than only combat deployments and exposures, and potential individual differences, interact to predict post-deployment changes in PTSS symptoms. However, less research has focused on which specific experiences of combat and deployment and post-deployment experiences affect particular core PTSD symptoms, nor how gains or losses among particular social and personal resources in the workplace may buffer or enhance posttraumatic symptoms. Nonetheless, evidence across multiple war eras and cohorts of U.S. veterans reveals that exposure to combat is an established risk factor for 
later onset of PTSD (e.g., Dohrenwend et al., 2013). One of many, apparently, that in isolation are insufficient to cause PTSD among a majority of combat veterans sharing comparable wartime experiences (e.g., Dohrenwend et al., 2013).

Hypothesis 1: Early theoretical research into fear-conditioning and behavioral learning (e.g., Mowrer, 1960) and clinical studies with Vietnam-era veterans showed that levels of warzone exposures (Kulka et al., 1990) and other post-deployment environmental processes were causally related to posttraumatic stress symptoms. The earliest models of PTSD (Mowrer, 1960) posited two factors related to stress reactions: (1) fear-conditioning; and, (2) behavioral avoidance (e.g., Brewin \& Holmes, 2003). Prior theory and research would predict that combat experiences may also be fearprovoking and thereby evoke perceptions of reexperiencing driving heightened arousal and behavioral avoidance under triggering environmental conditions and stimuli (e.g., loud noises, crowds, ambiguous social support) leading to PTSS. Therefore, (as shown in Figure 1.), according to theory, research, and logic the following is hypothesized:

Hypothesis 1: After controlling for demographic, individual, and militaryrelated factors, combat experiences (CES) will be positively related to PTSS.

Since Mowrer (1960) other stressor-strain configurations based on the Transactional Model of Stress and Coping (Lazarus \& Folkman, 1984) have informed work stress mechanisms such as resource loss spirals (Hobfoll, 1989) and the health impairment process and performance protection strategies (Bakker \& Demouruti, 2007). These later day work-stress process models provide underlying theoretical mechanisms to predict potential interactions and other indirect pathways to explain the influence of psychosocial and biological factors that link perceived stressors with 
physiological and psychological strain. As noted in comments by Sapolsky (2002), on a twin study of Vietnam-era twins discordant for warzone experiences, combat exposure was one of many risk factors for onset of PTSD that later research revealed was not necessarily causative. Therefore, a review of theories and research that informed proposed moderators of a link between combat experiences and combat exposures and PTSD and PTSS, both in the work environment and job situation as well as between individuals, was conducted to motivate the remaining hypotheses. 


\section{Integrated theoretical framework}

Among the most revolutionary models in the occupational stress literature are Karasek's demand-control model (Karasek, 1979) and the "Stress-Buffering Hypothesis" (Cohen \& Willis, 1985). The DCM provides a theory for predicting differing responses to job demands premised on the expected attenuating (i.e., "buffering") effects of high job control and high job demands that are theorized to moderate negative strain reactions (Karasek \& Theorell, 1990). In addition, the DCM also proposes how personality traits and behavior patterns at work contribute to the “adult socialization” process (Landsbergis, Schnall, Belkic, Baker, Schwartz, \& Pickering, 2011, p.252). For example, "chronic adaptation to low control-low demand" work conditions (i.e., "passive jobs” such as 'night watchman') are linked to lower selfefficacy, higher external locus of control, compromised problem-solving ability, depressed feelings and learned helplessness (Karasek \& Theorell, 1990).

In contrast to the job demands $x$ job control hypotheses, the "Buffering Hypothesis" (Cohen \& Willis, 1985) centers on associations among stress levels, coping, social support and adjustment. However, inconsistent results from empirical tests of the DCM's central tenet of the theoretical buffering effect of high levels of job control (e.g., Dwyer \& Ganster, 1991; Sargent \& Terry, 1998; Spector, 1987) have been attributed by some organizational scholars to conceptual and methodological flaws in prior research designs (Jimmieson \& Terry, 1999; Wall, Jackson, Mullarkey, \& Parker, 1996). Such limitations of the DCM and experimental research into the buffering hypothesis led to an extension of the DCM: The Job Demand-Control-Support (JDC-S) 
model (e.g., Van der Doef et al., 1999). According to the JDC-S, job demands, job control, and "worksite social integration" are relevant factors in "the development of health problems" (e.g., Van der Doef et al., 1999, p. 89). Therefore, an integrated theoretical approach to test hypothesized relations among demands, control, and support with working veterans would necessitate inclusion of a process by which these independent predictors interact, the sources from which such resources as control and support flow to the worker, and an explanation for the underlying mechanisms by which these factors influence health and well-being at both the physiological and psychological levels.

Moderation theory. The buffering hypotheses and its opposite (i.e., the enhancer hypothesis) are two forms of moderation analyses. While the buffering hypothesis predicts the expected attenuation of the main effect of a stressor (e.g., Combat Experiences) on a criterion variable (i.e., PTSS), the enhancer hypothesis predicts the exact opposite: the effect of the predictor on the criterion will be greater under higher levels of the moderator (i.e., iso-strain). Overall, the aim of moderation analyses is to expand a researcher's comprehension from does "X cause $\mathrm{Y}$ " to "under what conditions, or for whom does X cause Y” (e.g., Baron \& Kenny, 1986; Schimdt, 2011, p.58). Due to the greater frequency of work disruptions from taking military-related leaves of absences from their civilian jobs that reservists uniquely experience (e.g., Sayer et al., 2014), compared to military members serving in active components, it was expected that active reserve veteran employees (“ARVEs") would occupy "passive jobs" at higher rates than either fully separated active ("SAVEs") or separated reserve 
component veteran employees ("SRVEs"). This expectation of differences between veteran employee subgroups (VES) as well as how one's job situation and quality of sleep may differentially affect PTSS motivated an interest in the question of whether the interaction of CES $x$ VES moderated CES on PTSS as well as testing how the combined influences of job demands, job control, workplace social support (i.e., iso-strain) and an individual's quality of sleep may moderate symptoms of posttraumatic stress (PTSS) following experiences of combat.

Shortcomings of DCM and JDC-S models. Most job stress theories propose the "environment-response" concept whereby an environmental stressor is presumed to cause a strain response in the individual (Chang \& Spector, 2011). As additionally defined by Chang et al., (2011), stressors may be environmental stimuli that demand or provoke an adaptive response; whereas strains are reactions to stressors that may manifest as physical (e.g., heightened cortisol levels), psychological (e.g., anxiety), or behavioral (e.g., cigarette smoking) signs or symptoms. To test proposed moderators of Combat Experiences (i.e., "stressors") on PTSS (i.e., "strain") among veteran employees in the civilian workplace an applied framework where work situation factors, job characteristics, and individual differences predictably interact was required.

Both the DCM and JDC-S provide theoretical perspectives on several variables related to workplace conditions in the proposed study. However, limited evidence supports the DCM's proposed buffering effect of job control; and, contrary to Karasek's original interaction hypothesis between job demands and job control, more support has been found for the additive effect of demands and control on strain (Perrewe \& Ganster, 
2007). While a few longitudinal studies among homogenous occupational groups support Karasek's proposed demands x control hypothesis (e.g., Morrison, Dunne, Fitzgerald, \& Cloghan, 1992; Parkes, Mendham, \& von Rabenau, 1994), others indicate that between job control (i.e., "task autonomy") and skill discretion (i.e., "skill utilization"), a "demand-skill-support-model" best explained associations among "work characteristics, health, and well-being" (van Veldhoven, Taris, de Jonge, \& Broesen, 2005, p. 4). Further, the workload components of time and physical demands may be most related to "work-related fatigue" (van Veldhoven et al., 2005, p. 19). The potential association of workload with work-related fatigue has implications for the proposed investigation of how Iso-strain and deficient Sleep Quality may enhance or buffer combat stressors on PTSS while also potentially differing in relative strength and weakness between groups of veterans reintegrating into the civilian workforce.

With respect to the demands-control-support model, occupational health scholars have argued that the JDC-S may be "too simplistic for explanations" of "health and well-being" outcomes among certain subpopulations (Ganster et al., in Quick et al., 2013, p.43) such as among men (Vehoeven, Maes, Kraaij, Joekes, 2003, p.423) who may be more susceptible to iso-strain environments. As both the DCM and JDC-S lack specificity to predict how work situation resources (e.g., coworker/supervisor support, role clarity) and organizational resources (e.g., job control, effective job training) and individual resources (e.g., good sleep quality) may moderate symptoms of impaired healthy functioning among a sample of largely male combat veteran civilian employees, who may also be experiencing chronic symptoms of PTSD such as 
"reexperiencing", "avoidance", and "arousal", the proposed thesis integrated several theoretical perspectives. The aim of this theoretical integration was to inform specific hypotheses on how various proposed moderators may influence the strength of CES on PTSS. As a recent update to Karasek's and Theorell's DMC and Siegrist's (1996) Effort Reward Imbalance (ERI) model, the Job Demands-Resources (JD-R) model has become a major "job-stress" framework among organizational researchers (Demerouti et al., 2001) that provides extended theoretical possibilities for making predictions on the interplay among individual, work, and job-related resources that were relevant to this thesis study.

Job Demands-Resources Model (JD-R). Fundamentally, all three models (i.e., DCM, EFI, JD-R) assume that employee health and well-being are the result of the balance of resources (positive) and demands (negative) found within the workplace (Schaufeli \& Taris, 2014). Most organizational stress studies have taken a prescriptive (i.e., "laundry-list") approach by selecting models with a predefined range of predictors of employee health and well-being that may be irrelevant for many jobs (Bakker \& Demerouti, 2007). Only the JD-R presumes that any demand or any resource may impact employee health and well-being (Schaufeli \& Taris, 2014). As with the DCM, the JD-R framework operationalizes job demands as characteristics of a job that require sustained psychological (cognitive or emotional), physical effort or skills that exact “physiological and/or psychological costs" (Bakker \& Demerouti, 2007, p. 312). By contrast, job resources are "those physical, psychological, social, or organizational aspects" of the workplace or job that are either instrumental to performing it (e.g., water 
for a fire department), or decrease job demands and "the associated physiological and psychological costs" by promoting "learning, personal growth, and development" (Bakker \& Demerouti, 2007, p. 312). Additionally, the JD-R predicts (Figure 3.) that the interaction of job demands and job resources can lead to the development of unhealthy job strain or motivate healthy and adaptive work engagement (Bakker \& Demerouti, 2007).

While similar to the DCM, the JD-R model proposes two underlying biopsychosocial processes driving the development of job strain and motivation. (1) The health impairment processes (e.g., poorly designed jobs, chronic work overload) may exhaust mental and physical resources leading to health problems and strain, which in turn may activate the SNS (i.e., Sympathetic Nervous System). (2) Performance protection strategies may exact psychological (e.g., daytime sleepiness) and physiological costs (e.g., high blood pressure). Compensatory cognitive-behavioral strategies (e.g., shortened attention spans, redefinition of assigned tasks, Insufficient Sleep Syndrome) to offset SNS activation may lead to the draining of a worker's energy "resulting in a breakdown". Conversely, (2) Job Resources (e.g., social support, autonomy, feedback) are motivational, especially under high demands, and may promote work engagement, low cynicism, and excellent performance (Bakker \& Demerouti, 2007).

Interactions between job demands and resources. Under the JD-R (Figure 3.), the assumed effect of buffering is extended beyond job control and includes job resources, any of which (i.e., organizational or job specific) may buffer the effect of job 
demands on job strain (e.g., burnout). Further, as argued by Kahn and Byosserie (1992), buffering effects under the JD-R can occur between any two variables in the stressorstrain pathway that may emanate from the work situation (e.g., Workplace Social Support) or individual characteristics (e.g., Sleep Quality). Buffers may decrease the tendency of organizations to create specific stressors (e.g., job demands), change perceptions (e.g., perceived job control) or cognitions (e.g., guilty self-blame) provoked by work situation stressors (e.g., shiftwork). Buffers may also alter physiological responses following cognitive appraisals and decrease health-damaging outcomes such as PTSS (Kahn \& Byosserie, 1992, p.622). Lastly, instrumental support from coworkers may help complete job tasks on time and thus alleviate the effect of work overload on potential strain reactions (Van der Doef et al., 1999).

Support for JD-R. Tests of the main effects of job demands and resources using structural equation analyses of both self-report and observer ratings predicted exhaustion, burnout and engagement where job demands are associated with exhaustion and lacking resources in the work environment predicted disengagement (e.g., Bakker \& Demerouti, 2007, p. 316; Schaufeli \& Taris, 2014). Neuroscientific support for of the JD-R's health impairment process (e.g., Dinges, Pack, Williams, Gillen, Powell, Ott, et al., 1997; Frank, 2006) can be found in "Restorative and Brain Plasticity" models of sleep that indicate that disturbed sleep and the failure to initiate or maintain sleep, all symptoms of insomnia, may result from the interaction of sympathetic nervous system arousal and negative affect (e.g., "worrying"). Importantly, sleep loss, as experienced by insomniacs, may negatively impact job performance and result in increased 
workplace accidents, injuries, and loss of productivity (Kantermann, Juda, Vetter, \& Roenneberg, 2010). Also, Kahn and colleagues (1992, p.622) offered evidence supporting the predicted buffering effect of workplace social support attenuating healthdamaging consequences following negative appraisals of organizational stressors. For example, a newcomer veteran employee may experience work stress after perceiving that their new civilian job has low personal value, different customs and norms around social interactions and support-seeking, or more simply when attempting to maintain civilian employment by increasing their subjective work efforts while forgoing medical treatment for worsening deployment-related physical and psychological injuries. Embedded in the JD-R's theory of "job resources" are elements of Hobfoll's (1989) COR theory, which provides additional theoretical predictions (Bakker \& Demerouti, 2007) relevant to applied studies among military populations reintegrating into the civilian sphere.

According to COR theory resource loss is asserted as the main cause of the negative sequela of stressful conditions, including psychological distress, negative health outcomes, and diminished functioning. For example, losing one's job may contribute to losing one's house and becoming homeless, which may further erode one's psychological and physical health. Further, as Hobfoll later argued, the COR model is appropriate for studying general occupational as well as military stress as it has guided earlier research on war-related trauma in military samples (Hobfoll et al., 2012). Of significant interest to the present study, Westman, Hobfoll, Chen, Davidon, and Laski (2005) distinguished between "primary" and "secondary" resources. Primary 
resources can be food, shelter, positive well-being, sleep, and social connectedness. The function of secondary resources is to aid in creating and preserving primary resources such as time, credit, insurance, and work (Westman, 2001).

Linking employee status, combat experiences, and PTSS. In SERVe, veteran employees (VES) had returned seven years, on average, from their last deployment. Therefore, they were expected to differ on levels of PTSS by group membership (i.e., ARVES, SAVES, SRVES) and to have obtained employment with different levels of job control, which Luchman et al. (2013) showed was positively related $(r=.22)$ to social support. Moreover, prior research by Frese's (1999) revealed that CWS that matched a social dysfunction (e.g., irritation/strain and anxiety) acted as a stronger buffer than for psychosomatic problems (e.g., sleep, upset stomachs). Meanwhile, PTSS may erode social support over time and the combined effect of low workplace social support (WSS) may enhance symptoms of PTSS among combat veterans.

Research Question 2. The Allostatic Load and JD-R models along with post-9/11 population-level studies reveal a process behind how mental health symptoms of depression and PTSD differ dramatically between reintegrating active duty and reserve veterans. These theories and studies provide a rational basis for proposing a moderation hypothesis based in expected differences in perceived work stressors, job control, and social support generating allostatic state differences by group membership among veteran employee participants. However, due to the small sample size $(N=69)$ in the ARVE group, concerns over issues of sufficient statistical power $($ power $=1-\beta \geq .80)$ resulted in the decision to pose the interaction of CES $x$ VES as a research question and 
pilot test of the effectiveness of the proposed Bayesian method to overcome traditional constraints around power (i.e., $1-\beta \geq .80$ ) with small samples. Therefore, informed by Allostatic Load and JD-R theories, which imply that increased perceived stress may differentially activate effector systems and thereby accelerate the health impairment process and performance protection strategies, the following research question (Figure 1.) was proposed:

$R Q \# 2$ : After controlling for individual, demographic and military-related factors, does Veteran Employee Status (VES) moderate the main effect of CES on PTSS such that those endorsing affiliation with the active reservist group also score higher on PTSS relative to separated active and reserve veteran employees?

Job demands, control, support, and iso-strain. With the Demand ControlSupport (DCS) model, Karasek and Theorell (1990) extended the DCM to account for the influence of social support and high control in buffering the harmful effects of job demands (e.g., Chang \& Spector, 2011, p.122). However, the DCS does not stand alone. According to Bakker and Demerouti (2007) both the DCS and Effort-Reward Imbalance (ERI) models exhibit theoretical weaknesses due to "the limited range of predictor variables" that may be unrelated to all "job characteristics that workers experience" aside from job control and support that may moderate the negative impact of demands and strain. Nonetheless, the DCS is the most empirically tested and validated of all models. However, a recent metaanalysis (Luchman \& GonzalezMorales, 2013) tested the theoretical propositions of both the DCS and JD-R model while attempting to disentangle the interrelationships among the work characteristics of demands, control, and support. 
Results from Luchman and Gonzalez-Morales (2013) both supported and ran somewhat counter to expectations. Though the association between task-related demands and job control was hypothesized to be negative multiple analyses determined it was statistically insignificant; while the association of task-related demands and CWS was negative as predicted with an average moderate effect size $(r=-.11)$. Also, job control and CWS were, as predicted, positively related with an average moderate effect size $(r=.22)$. Ninety-five percent confidence intervals did not include zero and publication bias was not found to be a likely factor in these results (Luchman \& Gonzalez-Morales, 2013, p.42). Further, in the meta-analytic structural equation model CWS was negatively related to both task-related job demands $(r=-.11)$ and burnout $(r$ $=-19)$ and positively related to job control $(r=.23)$.

As a contribution to overall theory and practice, Luchman and colleagues' (2013) meta-analytic findings revealed that task-related demands and job control had no bivariate correlation; whereas, the moderating effect of job control and CWS on job demands was significantly negative. An interesting speculation for the original bivariate null finding of demands and control was revealed by a "between-subjects" moderator analysis. Apparently, the demand-control association interacts with gender such that female samples revealed mainly negative correlations while male samples showed positive correlations. Normally, Karasek's DCM (1979) would predict that high job control would act as a buffer against strain under high demands, but metaanalytic evidence suggests that gender may moderate the bivariate association between demands and control such that men are more likely to have more job control than women. As the 
majority of participants $(N>75 \%)$ in the SERVe sample were men, coupled with the finding that it may take up to a decade for veterans to recover from an initial "wage penalty" (Teachman, 2004), both theory and research predict that National Guard and Reserve veterans are far more likely to experience higher resource loss postreintegration compared to veterans without any concurrent military obligation. Moreover, as other evidence shows that active reservists have difficulties establishing careers (e.g., BLS, 2015; St John, 2015) it is unlikely that, all other factors being equal, the proposed subgroup of active reserve veteran employees would hold, on average, jobs with similar levels of control as the subgroup of military separated veteran employees.

Luchman and Gonzalez-Morales' metaanalysis (2013) provides evidence for the independent and predictive benefit of social support in the work environment from coworkers and supervisors such that workplaces with low demands, high control and supportive coworkers and supervisors are expected to produce the highest levels of well-being. Among other personal benefits, social support from supervisors and coworkers appears to be helpful in providing employees with a pool of social capital that may facilitate access to information, task assistance, or companionship and thereby reduce psychological and physiological stressors (Luchman \& Gonzalez-Morales, 2013). In the aggregate, CWS and supervisor social support may combine into a workplace social support dimension (WSS), measuring dual sources of potential workplace resources. High levels of WSS may attenuate a host of common psychosocial resource-loss spirals (e.g., quitting a job after being denied a promotion) as experienced 
among veterans enduring the negative effects of CES on PTSS during reintegration into the civilian work domain. Further, the JD-R states that resources may play an instrumental role in achieving work goals and fulfilling basic human needs (e.g., directly helping a colleague finish a task). Essentially, the JD-R predicts that the initial appraisal of demands and resources leads to strain when job demands exceed job resources (Bakker \& Demerouti, 2007).

As instrumental support from coworkers may lower actual or perceived work overload, on the plus side it may also lead to greater work engagement and facilitate the completion of more tasks associated with one's workload and thus lower arousal of SNS (e.g., Bakker \& Demerouti, 2007), allowing for replenishment of physical and psychological resources during non-work recovery hours (e.g., Sonnentag \& Fritz, 2011) and decrements in avoidance symptoms associated with PTSS. Prior research and theory suggests that job demands (e.g., work overload) will have a weaker impact on employees' quality and quantity of sleep under higher levels of support from coworkers. It is entirely possible, however, that the post-9/11 gulf between the majority of citizens who have not served in the U.S. military ( 99\%) and the effects of military service and deployments on the small minority who have served may prove to be too insurmountable (e.g., Taylor, 2011). That is, as was found in King and colleagues' retrospective study of veterans, PTSS may impair interpersonal relationships and erode the perceived availability of social support (King, Taft, King, Hammond, \& Stone, 2006). 
Linking Iso-strain to CES and PTSS. First defined by Karasek (1979), job strain results from the interaction between a person and their work situation when the combination of "high job demands" and "low control" or "decision latitude" produces strain reactions. In the current study, job demands were operationalized by "workload" (i.e., time pressure, difficulty of work), and "strain reactions" represented three of four dimensions of posttraumatic stress (i.e., re-experiencing, arousal, and avoidance). A vast literature of scholarship shows that workload correlates strongly with high-arousal, negative affect (e.g., anxiety), or nonspecific psychosomatic symptoms (e.g., upset stomach, migrating body pains), which by definition have no known underlying medical cause (Barlow \& Durand, 2002, p.162; Demerouti et al., 2001; Houkes, Jansen, deJonge, \& Nijhuis, 2001; Warr, 2007).

As active reserve veteran employees (ARVEs) in the current study essentially held at least two jobs (i.e., civilian + part-time military) it was expected that they would perceive higher job demands and workloads when self-reporting on civilian job stress measures. In addition, research suggests that due to complications inherent in establishing and advancing a civilian career while on active reserve status (e.g., St John, 2015), it was also expected that ARVEs would, on average, hold civilian jobs with less inherent decision latitude or job control. Coupled with their expected higher levels of PTSS, and as suggested by other research on the negative association of PTSD at Time 1 and social support at Time 2 (e.g., King et al., 2006), with all other background factors being equal, ARVEs were expected to be more likely to have alienated whatever perceived WSS was available at the start of their reintegration process. By contrast, the 
two other separated veteran employee groups were expected to have relatively lower perceived psychosocial job demands, in terms of perceived workload, by virtue of having no competing military obligations to postpone or compromise advancing further in their civilian careers relative to ARVEs. Thus, separated veteran employees were expected to have accrued greater, on average, levels of job control.

Hypothesis 2. According to JD-R theory and evidence from prior organizational research (Nieuwenhuijsen et al., 2010), which found that work situations characterized by iso-strain (ISO) and effort-reward imbalance "predicted stress-related disorders", higher levels of ISO were expected to activate the SNS and health impairment process longer, leading to higher perceived strain and PTSS. By contrast, WSS that matched a veteran's coping need and adequate job and schedule control were expected to produce higher vigor and work engagement and reduce veteran perceptions of the negative effects of CES on PTSS. Therefore, as shown in Figure 4., the following two-way interaction was proposed:

Hypothesis 2: After controlling for individual, demographic and militaryrelated factors, ISO will enhance the positive main effect of CES on PTSS such that under the condition of high CES those scoring highest on ISO will also, on average, score highest on PTSS relative to those scoring lowest on ISO.

Linking Sleep Quality to CES and PTSS. In an epidemiological review of research among Japanese workers, Doi (2005) found that work conditions related to “insomnia, poor sleep quality and deprived sleep” included such job characteristics as "heavy work load or overwork", "difficulty in job", "interpersonal problems", and "low 
social support”. Research on the link between occupational stress and disturbed sleep suggests that people performing shiftwork are more likely than those with regular work schedules to complain of fatigue and anxiety and report decreases in quality and quantity of sleep (Harrington, 2001, p.69). Additionally, high work demands coupled with effortful physical work are risk factors for disturbed sleep (Akerstedt et al., 2002a). Disturbed sleeping patterns connected with shift-work can result in "shift-work sleep disorder (SWSD)" where misalignment of the circadian rhythm, resulting from nightshift work, causes excessive sleepiness during night work or insomnia when trying to initiate recovery sleep the following day (Harrington, 2001, p.69).

Though a majority of nightshift workers have difficulty initiating sleep, "many compensate with temporal sleep-wake dysregulation". Between $5 \%$ and $10 \%$ of nightshift workers (about 6 million Americans) are unable to adapt their natural circadian rhythms and thus experience excessive daytime sleepiness and daytime insomnia meeting criteria for diagnosis of SWSD (Weilburg, Stakes, \& Roth, 2008, p. 298). Based on annual National Guard Bureau (NGB) "posture statements” (DoD/NGB, 2013/2015), it was anticipated that at least $10 \%$ of ARVEs in the overall subsample of Active Reserve Veteran Employees $(N=69)$ would hold civilian jobs typified by the DCM's "high-strain" category. Additionally, to the extent that ARVEs experience relatively higher conflicts over balancing their competing military and civilian work schedules, compared with the other two separated veteran employee groups, they were expected to experience shorter sleep durations. 
Hypothesis 3: Interestingly, occupational health researchers have explored biological (i.e., "biomarker") evidence of demand-control interactions on job strain following sleep. In a study of seventy-six British white-collar workers Rydstedt and Devereux (2013) reported significant interactions $\left[F_{(1,72)}=4.68, p<.05\right]$ between levels of job strain and sleep quality on morning saliva cortisol secretion such that the interaction between low sleep quality among workers with high job strain resulted in lower morning cortisol reactivity. Drawing on past research indicating that job demands such as workload and effortful work are risk factors for insomnia and poor sleep quality (Akerstedt et al., 2002b; Doi, 2005), it is likely that veterans who experience high workloads will engage in performance protection strategies such as greater subjective effort and therefore experience greater depletion of their physical and mental resources, higher SNS activation, greater levels of disrupted sleep, higher exhaustion and less sleep duration. As predicted by the 'feeling as information hypothesis', during presleep veteran employees experiencing high levels of iso-strain may also engage in rumination (i.e., "worry over work") and an inability to meet their workload demands (e.g., Barnes, 2012, Berset, Elfering, Lüthy, Lüthi, \& Semmer, 2011; Borders, McAndrew, Quigley, \& Chandler, 2012; Kompier, Taris, \& van Veldhoven, 2012). Chronic presleep rumination over work (e.g., National Guard employees juggling civilian and military employment) may differentially arouse allostatic effector systems and sympathetic nervous systems relative to veteran workers without a current military obligation. In turn, employees experiencing job strain may perceive high arousal of their allostatic effector systems as a hindrance stressor (e.g., a barrier to meeting goals) that delays 
rapid onset of sleep and energetic recovery from restful sleep; two symptoms of insomnia (Basta et al., 2007).

Thus, iso-strain employment may promote prolonged sleep latency and greater daytime sleepiness, which in turn may form a vicious cycle of objective and progressive resource loss spirals. Resource loss spirals may start as reduced work output before progressing to negative performance appraisals, resulting in loss of relative wages and decreased supervisor support that culminates in opportunity costs ranging from denial of promotions to higher-paying jobs and enriching career opportunities in more supportive work environments. Based on the theoretical mechanism of the health impairment process and the logic of higher job demands activating the SNS and performance protection strategies, it is proposed that increased subjective work effort and non-work rumination (i.e., "worry over work") will trigger poor sleep quality, in the absence of sufficient organizational resources, due to increased sleep latency and other symptoms of insomnia. Therefore, the following two-way interaction (Figure 5.) was hypothesized:

Hypothesis 3: After controlling for demographic and military-related factors, greater Sleep Quality (i.e., low scores = good sleepers) will significantly and positively buffer the main effect of CES on PTSS such that those scoring lowest on SLEEP under high CES will also, on average, score lower on PTSS relative to those scoring higher on SLEEP.

The proposed hypotheses and research question were analyzed by way of a progressive series of hierarchical multiple regression and structural equation models (SEM) utilizing both frequentist and Bayesian estimators and optimizers (e.g., Gibbs algorithmic "Random Walk" resampling of the posterior predictive distributions) of 
the modeled statistical associations among the focal variables. Essentially, the goal of these additional analyses was to test the proposed hypotheses twice by different analytic techniques while also minimizing risk for Type I and Type II errors due to uncorrectable non-constant residual error variance and violations of the assumption of multivariate normality among the focal predictors of CES, Iso-strain, and Sleep Quality. 


\section{Methods}

\section{Participants and Procedures}

The current thesis study drew upon baseline data $(N=497)$ from the larger, fiveyear DoD-funded SERVe project, a randomized-control treatment study of post-9/11 veterans. Between 2013 and 2015, SERVe researchers, principal investigators, project managers, consultants, and research assistants recruited more than eleven public and private industries from the Pacific Northwest to participate. This author also engaged in veteran recruitment and outreach by promoting SERVe at annual medical screenings for Oregon National Guard soldiers. In order of prevalence organizations recruited into SERVe represented workers in such diverse industries as: government; manufacturing; education/health services; information; construction; professional/business services; trade/transportation and utilities. Through its customized intervention modules (i.e., "Veteran Supportive Supervisor Training") SERVe aims to improve the health, wellbeing, and employment stability of post-9/11 veterans and their families by training their civilian supervisors on how to be more behaviorally supportive of their veteran employees (SERVe website, 2013).

Participants. Officially, the U.S. government considers a "veteran" as one who served in the active U.S. military, Coast Guard, uniformed Public Health Service, and the uniformed National Oceanic and Atmospheric Administration, reservists called to active duty, and those disabled while on active duty training. In general, service members who have not separated from active duty, or received a dishonorable discharge, or whose active duty service consisted only of training are considered 
ineligible for "veteran" benefits (Westat, 2010). For the purposes of this thesis the term "veteran" was applied to any SERVe participant who, since 9/11, self-identified as having served on active duty military orders and was "discharged or released" from active duty "under conditions other than dishonorable". In addition, this definition of "veteran" applied to active or separated reservists who self-reported: (a) already deployed and/or left military service; or, (b) were still serving in the Air or Army National Guard or one of the five major reserve components of the U.S. military after completing a tour of active duty. This thesis study's definition of "veteran" is consistent with federal regulations (GAO, 2014, p.30). In the current analyses, only SERVe participants endorsing at least one combat experience were included. This reduced the final subsample to less than four-hundred $(N=382)$ veteran employees.

Baseline descriptive statistics. As shown in Table 1., self-identified membership among the three veteran employee subgroups (i.e., Active Reserve Component ["Act Reserve"], Separated Active Duty [“Sep Active"], Separated Reserve Component [“Sep Reserve"]) was uneven (i.e., Act Reserve $=69 ;$ Sep Active $=185$; Sep Reserve $=128$ ). In addition, five covariates were included as control variables: Personality (i.e., conscientiousness, neuroticism); Socioeconomic Status (SES); Risk of Homelessness (HOME); and Reintegration Time (i.e., time since last deployment or "dep time"). Analyses of the five covariates revealed significant group differences at baseline. On average, Separated Active Veterans had obtained higher levels of education $(M=$ $5.18 / \mathrm{sd}=2.57, p<.05)$. 
While, on average, Separated Reserve Veterans had reintegrated longer - as measured by months since their last deployment $(M=98.4 / \mathrm{sd}=42.47, p<.05)$. Finally, though no veteran employee subgroup reported significantly higher levels of Combat Experiences, Separated Active Veteran employees did endorse significantly higher levels of PTSS $(M=8.6 / \mathrm{sd}=4.52, p<.05)$ at baseline relative to the other two veteran employee subgroups. In total, the SERVe baseline sample of combat veterans was mostly male $(89.3 \%)$ and white $(80.9 \%)$ with a mean age of about 38 years $(M=38.4)$.

Electronic survey study design. At the core of the SERVe project's panel study design is a multi-wave, self-reported electronic survey-based data collection method to recording participant responses to a range of organizational, health, well-being, interpersonal, military, demographic, individual, and work-family measures. Qualtrics software was used to construct survey items that were delivered electronically in nine periodic "bursts" (i.e., "waves") over a 12-month timespan via e-mail to veterans, civilian supervisors, and veteran spouses. Before inclusion within the baseline dataset, individual surveys were de-identified and downloaded in Excel spreadsheets, which were stored on a password protected server in compliance with intuitional review board policies and health privacy laws. Conceptually, while there are theoretically interesting multiple levels to analyze within the military reintegration and work-family interface, this thesis investigated individual and group level units of analyses.

\section{Measures}

Combat experiences. (Combat Experiences Scale, Vogt, Samper, King, King, \& Martin, Walter Reed Army Institute of Research [WRAIR], 2008). The full 33-item 
Combat Experiences Scale (CES) indexes war-related traumas, stressors and experiences across three dimensions (i.e., combat environment, proximate engagement, nearness to injury/death). According to its creators, development of the CES was a response to "the new kinds of combat and resulting psychological sequelae" of the present war era that necessitated a "more subjective measure" of combat at the individual level (Guyker, Donnelly, Donnelly, Dunnam, Warner, Kittleson et al., 2013). On recommendation from WRAIR consultants, the SERVe project utilized 27 of 33 items from the full CES measure that probed veteran employees' memories and recollections of perceived threats, experiences of violence, and the potential killing or harming of civilians and enemy combatants while on their most recent deployment. As shown in Table 3., after performing a confirmatory factor analysis (CFA), all 27 items recommended by WRAIR were retained and anchored to the statement, "During your deployment were you/did you ever? Example items included: “(1) Attacked or ambushed?: (2) Receive small arms fire?: (3) See dead bodies? (4) See dead or seriously injured Americans?" Items were scored from 1= "Never" to $4=$ " 5 or more times" and summed to formulate a global CES score, which produced a positively skewed univariate distribution. In the current sample for which data was available ( $N=377$ of 382) the 27 CES items exhibited excellent reliability $(\alpha=.95)$ sufficient for social scientific research.

Iso-strain standardized. After reviewing the research literature on prior iso-strain (ISO) measurement schemes, ISO was conceptualized as an adapted composite measure based on an algorithm from Mather et al. (2015) who found that job strain and iso-strain 
were risk factors for sick leave due to mental illness among Swedish birth twins. In order to capture the variance of high and low schedule control, which was expected to differ between active reservist employees and separated veteran employees, a global measure of schedule control (Thoman \& Ganster, 1995) was added to Mather and colleagues' algorithm.

The proposed configuration of the tri-dimensional iso-strain composite measure was theorized to tap the relative influences of job demands, job control, schedule control, and social support at work. Global ISO scores were derived by computing the following three-step algorithm (adapted from Mather et al., 2015):

Where,

(Step 1). Iso raw score $=$ Job demands $-($ decision latitude + schedule control $)-$ (supervisor support + coworker support)

(Step 2). Iso raw score + highest negative score $=$ Iso positive score (Step 3). Iso positive score/scaling constant = Iso-strain (standardized) In total 14 items were retained from: The Job Content Questionnaire (Karasek et al., 1998); General Coworker Support Scale (Caplan, Cobb, \& French, 1975); General Supervisor Support scale (Yoon \& Lim, 1999) and Control Over Work/Schedule Control scales (e.g., Thomas \& Ganster, 1995). Items were summed to create global component measures, which were then subtracted and recomputed to create a single, positive standardized score with higher scores indicating greater levels of iso-strain. The items were anchored to such statements as: (1) "Your job requires very hard work?" (rated 1-5); (2) "Your job allows you to make a lot of decisions on your own?" (rated 1-5) (3) "How easy is it to talk to your coworkers?" (rated 1-4); (4) "My 
supervisor does not really care about my well-being?" (rated 1-5); (5) "How much choice do you have over when you begin and end each work day?" (rated 1-5). Univariate inspection of the distribution of the ISO global score revealed a normal distribution. In the current sample for which data was available ( $N=377$ of 382$)$ the ISO composite measure exhibited excellent reliability $(\alpha=.82)$ for social scientific research.

Sleep Quality Reduced. Theoretically, in various combinations, five items can tap five of seven dimensions of Buysse and colleagues' (1989) Pittsburgh Sleep Quality Index (PSQI). Sleep disturbances (i.e., the mean of sleep latency plus fragmented sleep) and subjective sleep quality, all symptoms of insomnia, in addition to sleep duration, sleep efficiency, and sleep latency were measured using five items (i.e., sq_1, sq_2, sq5Rx_rc, sq6Rx_rc, sq7Rx_rc) from the twenty-five current PSQI items. For sleep duration participants were asked: (1) "Over the past month, what time did you usually turn the lights off to go to sleep?" (i.e., sq_1); and, (2) “Over the past month, what time did you usually get out of bed? (i.e., sq_2)" A sleep duration score (i.e., sq_4_sum) was obtained by computing the time difference between these two items (i.e., sq_2 minus sq_1) that was reversed scored and recalibrated with lower differences resulting in higher values (0-3). Sleep efficiency (i.e., hours slept/hours in bed x 100) was calculated by using two items (i.e., sqhr_slp, sqhr_bed) asking participants: (3) "During past 30 days, how many hours do you think you actually slept each day? This may be different than the number of hours you spent in bed." Estimated "hours spent in bed" was obtained by subtracting item 1 from item 2 (i.e., sq_2 minus sq_1). Sleep efficiency percentiles were recalibrated as follows " 0 " = " $>85 \%$ " to " 3 " = " $<65 \%$ ". Sleep 
disturbances were measured by two items (i.e., sq6Rx_rc, sq7Rx_rc) asking participants the following questions: (3) "During the past month, how often could you not get to sleep within 30 minutes?" And, (4) "During the past month, how often did you wake up in the middle of the night or early morning?" Items were rated on a scale from 1 (never) to 4 (three or more times a week) then summed and averaged with higher scores indicating greater sleep disturbances. For subjective sleep quality (i.e., sq5Rx_rc) participants were asked: "How often during the past month did you get enough sleep to feel rested upon waking up?" Items were rated on a scale from 1 (very bad) to 4 (very good) that were then reverse scored and recalibrated with higher scores (0-3) indicating worse sleep quality. Finally, all five components (i.e., subjective sleep quality, sleep latency, sleep duration, sleep efficiency, and sleep disturbances) were summed to create a global sleep quality reduced score (i.e., PSQI_5) with higher scores indicating worse sleep quality (i.e., "poor sleepers"). The remaining PSQI components of (6) use of sleep medication and (7) daytime dysfunction were not measured. In the current sample, Sleep Quality Reduced exhibited excellent reliability $(\alpha=.84, \omega=.91)$ for social scientific research.

Posttraumatic stress symptoms (PC-PTSD, Bliese, Wright, Adler, Cabrera, Castro, $\&$ Hoge, 2008). PTSS was measured by way of 4 of 17 items from the older PTSD Check List - Military (PCL-M), originally validated by Weathers, Huska, and Keane (1991), and recently revalidated by Bliese and colleagues (2008). Using Item Response Theory analyses, Bliese et al. (2008) created the brief form clinical scale known as the "Primary Care Posttraumatic Check List (PC-PCL)" that was the psychometric 
instrument to measure symptoms of PTSD in SERVe. In 2013, three new symptoms related to "negative alterations in cognitions and mood", creating a fourth dimension in addition to "intrusions/reexperiencing, avoidance, and alterations in arousal and reactivity," were added to the latest clinical model of PTSD (APA, 2013, pp.271-280). ${ }^{\text {iii }}$ Due to the parent study's (SERVe) selection of the validated PC-PCL instrument (Bliese et al., 2008), this fourth dimension of PTSD was unmeasured while three of four self-reported clinical symptoms of PTSD (i.e., reexperiencing, arousal, and avoidance) were measured. Items in the PC-PCLM were anchored to the following statement, "Below is a list of problems and complaints that veterans sometimes have in response to stressful life experiences. Please read each one carefully, put an ' $X$ ' in the box to indicate how much you have been bothered by that problem in the last month". The four items are: (1) "Repeated disturbing memories, thoughts, or images of the stressful experience?" (2) "Having physical reactions (like heart pounding, trouble breathing, sweating) when something reminded you of the stressful experience"; (3) "Avoiding activities or situations, because they remind you of the stressful experience"; (4) "Having difficulty concentrating". Items were scored on a 5-point scale and then summed with higher scores indicating greater probability of PTSD (Bliese et al., 2008). In the current sample $(N=382)$ for which data were available the PC-PCLM exhibited excellent reliability $(\alpha=.92, \omega=.94)$ for social scientific research.

Control variables. Prior military reintegration research (e.g., Bliese et al., 2011; Kulka et al., 1990) and a post-hoc correlation analysis of ten candidate factors informed the final selection of control variables. As shown in Table 4., five control variables were 
selected: Socioeconomic status (SES), as indicated by educational attainment, last military rank, and perceived financial strain; Time Since Last Deployment ("deptime") as indicated by months since last deployment; Risk of Homelessness (HOME) as indicated by a retrospective and prospective measure of homelessness; Conscientiousness (IPIPC) and Neuroticism (IPIPN). Together, these five control variables exhibited poor to adequate reliability $(\alpha / \omega=.43-.75)$ for social scientific research.

\section{Subgroup moderation and multigroup analyses}

According to SERVe recruitment protocols, participants within the third identifiable veteran subgroup of former reservists (i.e., "separated reserve veterans") were recruited from the same Pacific Northwest region as the two other veteran subgroups (i.e., "active reserve veterans, separated active veterans"). Interestingly, a majority of SERVe participants were recruited from within Oregon, a state with no major active duty military base or brigade-level units.

Over the last thirteen years Oregon's part-time military reservists have experienced and endured repeated combat deployments (Oregon Department of Veteran Affairs, 2016) at a tempo rivaling their active duty counterparts stationed on military posts in neighboring states (e.g., Joint Base Lewis-McChord in Washington state).

Consequently, it was theorized that this third group (i.e., "Separated Reserve Veteran Employees") likely shared many common cultural and military experiences with the ARVEs (Active Reserve Veteran Employees) group. Motivated by such theoretical considerations, a rationale to identify members of this third group of separated reservist 
employees prior to the main analyses became apparent in order to meet the originally proposed goal of directly comparing only active reserve veterans with separated former active duty veterans. However, considerations of reduced power from excluding the SRVEs group resulted in the final decision to retain them as a third self-identified veteran employee group. To achieve this, a scheme to decompose the "reserve veteran" group into those participants who self-identified their most recent military affiliation with either one of the five reserve components, including the Air and Army National Guard, was cross-referenced against those who indicated they were currently either "active" or "separated" military was applied. By creating a third factor level to the "Military Status" indicator variable (i.e., "Separated Reserve Component Veteran Employees" or "SRVES") the goal of identifying this third combat veteran employee subgroup was accomplished. 


\section{Analytical framework}

In the manner described by Martz, Bodner, and Livneh (2009), a moderation instead of mediation model was chosen for these cross-sectional data. Conceptually, after controlling for demographic, military, and personality factors, iso-strain and sleep quality were viewed as affecting the strength and direction of the association of combat experiences (CES) on posttraumatic stress symptoms (PTSS). Essentially, a mediation model would have implied that CES indirectly influenced PTSS through iso-strain and subjectively perceived quality of sleep. The main argument against mediation was that iso-strain and sleep quality are conceptualized as "statelike" constructs operating in flux rather than stabler "trait-like" constructs. Therefore, iso-strain and sleep quality were expected to affect background traumatic experiences on pathological strain reactions. As such, iso-strain and sleep quality are considered modifiable and therefore amendable to occupational health interventions.

Hypothesis testing. The analytical approach this thesis adopted consisted of a battery of hypotheses testing across a progressive series of nested multiple regression and SEM frameworks (i.e., hierarchical multiple regression and SEM/path analyses with frequentist, non-parametric, and Bayesian estimators) to determine whether sufficient statistical evidence at an exploratory alpha level $(\alpha<.10)$ was derived in support of the proposed hypotheses and research questions. The goal of these modeling frameworks and estimation methods was to apply a range of traditional and advanced quantitative methods to the task of detecting potentially subtle psychological effects that may elude conventional regression analysis. 


\section{Exploratory Data Analyses}

Achieving technically correct data. After eliminating all veteran participants $(N=115)$ from the SERVe baseline data set who did not endorse at least one combat experience, a final baseline sample of combat veterans $(N=382)$ was analyzed. Preliminary exploratory analyses revealed several technical problems to be addressed: (1) inspecting whether missing values were missing at random; (2) dealing with a missing data pattern at the item level (Dong \& Peng, 2013) for one baseline participant (i.e., Bayesian multiple imputation in Mplus 7.2 on the subsample of 382 combat veteran $^{\mathrm{iv}}$ ); (3) merging the imputed variable with the baseline dataset of combat veteran employees ( $N=382)$ in $R$; (4) correcting erroneously coded items and subscales within global scales (e.g., Pittsburgh Sleep Quality Index); and (5) creating new variables (e.g., Socioeconomic Status by multiplying subscale scores of Military Rank $x$ Educational Attainment $x$ Perceived Financial Strain). Upon making these technical corrections, and concluding that the mechanism of missing data ${ }^{\mathrm{v}}$ was either missing completely at random (MCAR) or missing at random (MAR) and therefore ignorable (e.g., (Little \& Rubin, 2002), this author proceeded with exploratory data analysis (EDA).

Before proceeding with this thesis's primary analyses, a series of EDAs were conducted in Mplus 7.2 and the open-source statistical software program $R$. Exploratory analyses were performed to test the assumption of multivariate normality and unidimensionality among all measures of focal and control variables. Lastly, to address the first research question (RQ\#1), a test of measurement invariance across three veteran 
employee subgroups on both the Combat Experiences Scale (CES) and the PTSS (PCPTSD) scales (Tables 7. \& 8.) were conducted to provide a final estimate of the degree of confidence in any significant findings of group mean differences were not the result of differences at the item-to-factor loading or unique error variance levels of these two focal constructs. Data were examined to determine the coding accuracy of focal and multicomponent measures (e.g., Sleep Quality), and whether the regression assumptions of multivariate normality, linearity, and homoscedasticity of residuals were met by interpreting Q-Q and residual scatter plots as well as by conducting formal tests of normality (i.e., Anderson-Darling and Kolmogorov-Smirnov) on each continuous focal and control variable. Also, because interactions were modeled, a multiple regression of all predictors was run in the open source program " $R$ ", using the statistical package "rockchalk", to test if multicollinearity among key variables was significant.

Multivariate normality. A key assumption of multivariate regression and structural equation modeling analyses is the assumption of multivariate normality of all variables. Inspection of the univariate distributions of the global scales of all focal and control variables (i.e., CES, ISO, Sleep Quality, PTSS, socioeconomic status (SES), Risk of Homelessness (HOME), Time Since Last Deployment, and the personality variables (Conscientiousness and Neuroticism) were conducted on the subsample $(N=382)$ of combat veterans only. According to Cohen and Cohen (2003, pp.237-238) statistical estimation of transformation parameters using the Box-Cox algorithm (e.g., Atkinson, 1994, Cohen \& Cohen, 2003, p.237; Draper \& Smith, 2014, p.280) can 
include maximum likelihood estimation to mathematically optimize the value of the transformation parameter " $\lambda$ ".

Additionally, computation of a maximum likelihood confidence interval around " $\lambda$ " can also provide a test of whether transformation to achieve normality is necessary. If the maximum likelihood confidence interval ("LL") includes the value " $\lambda=1$ ", then transformation is unnecessary as any untransformed raw score raised to the power of " 1 " is equal to the original untransformed raw score (Cohen \& Cohen, 2003, p.238). The main advantage of using Box-Cox transformations to normalize non-normally distributed variables over other transformation methods (e.g., “Tukey’s Ladder of Powers") is that the Box-Cox method simultaneously aims to produce "linearization, homoscedasticity, and normality of residuals" of variables with skewed distributions while also directly attempting to correct for the inherent instability of their variance (Cohen \& Cohen, 2003, p.246; Tukey, 1977). Briefly, a "residual" is the "error of prediction", or the difference between the predicted value of " $\hat{Y}$ " and the observed value of " $Y$ " $(Y-\hat{Y})$ with the goal of finding the line (the set of $\hat{Y})$ that minimizes such residual errors using the ordinary least squares (OLS) formula that minimizes the sum of the squared errors $\sum(Y-\hat{Y})^{2}$. In the present case, $\hat{Y}$ is the expected value of the PTSS score as predicted by the equation below for a given level of combat experiences, iso-strain, sleep, or the interactions of iso-strain and sleep quality along with the five control variables.

Where, 
Combat Experiences, Iso-strain, and Sleep Quality Affect PTSS

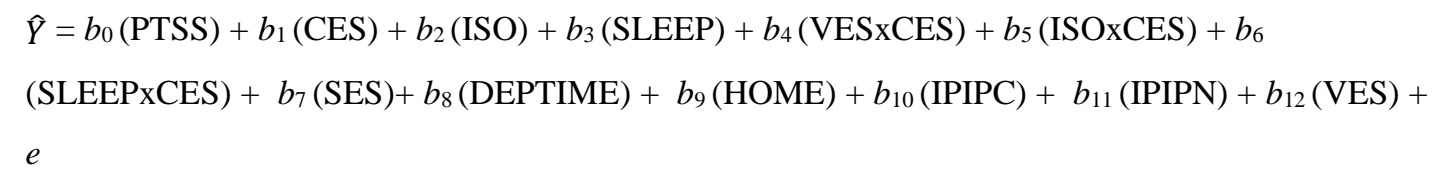

For these reasons, the Box-Cox approach to normalization was selected. ${ }^{\mathrm{vi}}$ Ultimately, however, due to the extreme non-normality of the sample data for CES, as indicated by the Lilliefors $(D=0.1962, p<0.000)$ and Anderson-Darling tests $(A=22.346$, $p<0.000)$, and PTSS, $(D=0.17215, p<0.000)$ and $(A=18.120, p<0.000)$, the Box-Cox transformations were largely unsuccessful in transforming these two focal variables to normality. In the case of the SES variable, the Box-Cox transformation led to a reordering of scores. In consideration of these various analytical challenges, attempts to transform non-normal and positively skewed univariate sample distributions to achieve greater normality were abandoned. Instead, it was decided that point estimates on hypothesized associations derived from classical regression analyses would be directly compared with those derived from SEM using Bayesian estimation formulae ${ }^{\text {vii }}$.

Multicollinearity and reliability analyses. According to Cohen and Cohen (2008, p. 423) calculation of the "Variance Inflation Factor (VIF)" [VIF $\left.=\left(1 / 1-\mathrm{R}^{2}{ }_{i .12 \ldots(i) \ldots k}\right)\right]$ for each term in a regression equation, excluding the intercept, provides a test of multicollinearity. A common heuristic is that any VIF $\geq 10$ is evidence of "serious multicollinearity" of the corresponding independent variable (Cohen \& Cohen, 2008, p.423). Such a finding of significant inflated variance provides a rationale for eliminating any term with a VIF $\geq 10$ from a final regression equation. An inspection of the range of VIF factor scores for the $27 \mathrm{CES}$ item terms regressed onto the CES global score $\left(V I F_{\text {CES }}=1.62-3.31\right)$ revealed no CES item term exceeding a VIF score $\geq 10$. 
Therefore, all 27 CES items were retained for further factor analyses. Next, multicollinearity among all predictors (i.e., Combat Experiences, Iso-strain, Sleep Quality, SES, Risk of Homelessness, Time Since Last Deployment, Conscientiousness and Neuroticism) was conducted by regressing these eight IVs onto the dependent variable (i.e., "PTSS"), which revealed no IV with a corresponding VIF $\geq 10$.

However, while performing internal reliability checks on all focal variables with the full baseline sample of veterans ( $N=512$ ), the Pittsburg Sleep Quality ("PSQI") measure initially produced an internal consistency estimate $(\alpha=.596)$, which was below what is generally considered reliable $(\alpha \geq .70)$ for social scientific research. This initial result raised suspicions of a coding error, because the PSQI has typically produced good reliability estimates in previous research with non-clinical samples (Mollayeva, Thurairajah, Burton, Mollayeva, Shapiro, \& Colantonio, 2015). Tasked with inspecting the coding schemes of each PSQI item, an undergraduate research assistant discovered that the "psqi4_v" item was mistakenly reverse coded in the baseline data set. According to scoring and coding instructions by the developers of the PSQI (Buysee et al.,1989) this coding error was corrected by undoing the erroneous reverse coding. Next, implementation of Buysee and colleagues' latest PSQI coding, recalibration, and scoring schemes (Buysse et al., 1989/2004) on the final reduced and imputed sample dataset ( $N=382)$ improved PSQI's internal reliability $(\alpha \geq .73)$. After obtaining the results of additional reliability checks conduced in $R$ ("psych" package), inspection of the SERVe codebook, and reviewing published instructions from the scale developers, one item (“jcq3R_v") from the Job Content Questionnaire (Karasek et al.,1998) was 
found to be negatively correlated with the item sum total only when included in the IsoStrain composite scale $(\alpha=.76)$. Also, two other personality variables ("ipip1_v" and “ipip5_v”) from the Mini-IPIP Scales (Donnellan, Oswald, Baird, \& Lucas, 2006) were negatively correlated with the item sum totals of their respective subscales' (i.e., "Conscientiousness", $\alpha=.50$, and "Neuroticism", $\alpha=.71$ ). These three items were reverse coded prior to the main analyses. Oddly, following these corrections, the reliabilities of the Iso-Strain $(\alpha=.93)$ and Conscientiousness $(\alpha=.51)$ and Neuroticism ( $\alpha=.71)$ scales incrementally improved .01 on one variable (Conscientiousness). After inspecting the Spearman-Brown prophecy formula for "ipip1_v", a decision to drop this item was made before rescoring the IPIPC subscale. This procedure resulted in some improvement $(\alpha=.05)$ of the reliability of the Conscientiousness measure $(\alpha=.55)$.

Additionally, during the data cleaning stage, it was discovered that the "Combat Exposure" measure (Weathers et al., 1989), listed in the SERVe code book, was in fact a similar-sounding but more recently published measure known as the Combat Experiences Scale (WRAIR, 2008) developed by U.S. Army psychologists. In total, in order to maintain consistency across all data sets associated with the parent study, these exploratory findings and the remedies taken were communicated to SERVe project managers. In addition to achieving technically correct data, several measurement issues also presented a challenge.

Internal Discussion on the Combat Experiences Scale. While "cleaning" the baseline data a discussion among the SERVe study's methodological scientist (Co-PI Dr. Todd Bodner, Ph.D.), this thesis's committee's methodological expert (Dr. Joel 
Steele, Ph.D.), and the present author ensued on the factor structure of the relatively new CES measure. Essentially, the discussion centered on whether or not CES was best represented as a "reflective" or "formative" construct. Drs. Steele and Bodner advised utilizing "obliquely rotated factors" and the construction of a formative factor model under the theory that "combat experiences" were unlikely to be orthogonal (i.e., independent) and most likely occurred in an environment where the factors would be correlated and best represented by allowing the manifest items to affect their latent factors (Kline, 2011). Briefly, the discussion how best to represent the Combat Experiences Scale involved the following notions and assumptions. Conceptually, the combat environment for U.S. troops in the post-9/11 era has been defined by the U.S. government as an "asymmetrical one" where conventional American military global dominance will motivate "potential adversaries to develop" unconventional "doctrines and methods" designed "to deter or neutralize US intervention" and "inflict massive damage on the homefront" (Haass, 2007/2008). Therefore, the direction of causality for the CES was hypothesized to be from the various manifest indicators and nonindependent dimensions of the combat environment through the CES measure.

Therefore, CES may be best conceptualized with both correlated (“oblique”) factors and the structure of the construct may be best represented as formative, where indicators inform the latent construct, rather than reflective whereby "latent combat experiences" are reflected in intercorrelated items. For example, while it may be argued that some combat experiences (e.g., surviving an ambush) cause "the seeing (of) dead bodies", as would be implied by a reflective construction of the CES, the multiple 
causes of "dead bodies" are independent of whether one witnesses "seeing dead bodies" after "surviving an ambush". After an exhaustive factor analyses process, the CES scale (Figure 6.) was determined to be best conceptualized as a multidimensional formative factor model comprised of four testlets.

\section{Exploratory and confirmatory factor analyses}

Factor analyses. Before proceeding with the main analyses related to the research hypotheses and questions, exploratory (EFA) and confirmatory factor analyses (CFA) were conducted to investigate the composition of Combat Experiences, Iso-strain, Sleep Quality, and PTSS items. Specifically, the goal of factor analyses was to identify the optimal number of common factors underlying each focal construct while eliminating items that did not meet a threshold loading value $(\geq .40)$ onto the common factors. After establishing sufficient reliability (Table 4.) for the CES $(\alpha=.95)$, ISO $(\alpha=.82)$, SLEEP $(\omega=.84)$, and PTSS $(\omega=.94)$ scales, a factor analysis (Table 4.) on the 27 CES items was conducted to identify the underlying dimensions of variability. The sample sizes for both the EFA and CFA were 382 working post-9/11 veterans who endorsed at least one combat experience.

Exploring the Combat Experiences Scale. A prior validity study of the Combat Experiences Scale (CES) using data from military personnel indicated that a threefactor reflective solution (i.e., Factor 1, "Combat Environment"; Factor 2, "Proximate Engagement"; Factor 3, "Nearness to Injury/Death") best fit the data (Guyker, Donnelly, Donnelly, Dunnam, Warner, Kittleson et al., 2013). However, though Guyker and colleagues (2013) conducted both principal and exploratory factor analyses the 
authors did not provide fit indices from a CFA to assess how well their final theoretical model fit their data. An inspection of the CES items (Table 2.) revealed depictions of diverse warfighting scenarios and facets of the combat environment that probed participants to reflect upon the frequency and intensity of their warzone experiences after reading such provocative descriptions as: "Receiving small arms fire"; "Improvised explosive devices/booby trap exploded near you"; "Being attacked or ambushed". A formative factor perspective might argue that for most combat veterans their experiences of combat occurred simultaneously or in proximity to one another and rarely in isolation. Moreover, these collective combat experiences were the logical consequences of precipitating stressors and/or stimuli that formed a gestalt.

Therefore, as shown in Table 3., the first step was to conduct an initial exploratory factor analysis using Mplus (7.2) with “oblimin" (i.e., oblique) rotation. The initial EFA consisted of three to four factors with 19 to 27 items that produced no fit indices meeting $\mathrm{Hu}$ and Bentler's (1999) recommended combinatorial cutoff criteria rules [(.96 $\geq \mathrm{TLI}$ and $\mathrm{SRMR} \leq 0.09$; or RMSEA $\leq .06$ and $\mathrm{SRMR} \leq 0.09$; or $.96 \geq \mathrm{TLI}$ and $\mathrm{SRMR}$ $\leq 0.09$ )] for covariance models exhibiting adequate fit to the data. However, EFA fit indices for a four-factor solution with $27 \mathrm{CES}$ items produced the closest best fit $\left[\left(X^{2}=851.59 ; d f=249 ; \mathrm{CFI}=0.901 ; \mathrm{TLI}=0.860 ; 90 \%\right.\right.$ RMSEA $(0.074,0.085) ;$ AIC=19064.933; $\mathrm{BIC}=19680.418 ; \mathrm{BICadj}=19185.457 ;$ SRMR=0.037)]. Correlations among the four factors ranged from $r=0.437-0.554$, and the sum of the estimated residual variances $\left(1-S_{\mathrm{r}}^{2}\right)$ revealed that $\sim 87 \%$ of the variance in CES was explained by the 27 items. 
Inspection of item content suggested that: Factor 1 (items) involved "Proximate Engagement in Combat"; Factor 2 (items) involved "Nearness to Injury or Death"; Factor 3 (items) involved "Threats"; and Factor 4 (items) involved "Combat Environment". For theoretical reasons, the decision to replicate Guyker and colleagues' (2013) three-factor CES solution was abandoned to proceed with testing a formative CES measurement model with the optimum number of independent "testlets". This necessitated grouping and summing items that loaded onto factors from the CFA into factor "parcels" before proceeding with constructing a formative CES model.

Reflective vs. formative combat experiences. The choice of whether a latent construct is specified as "reflective" or "formative" is far from trivial. Misspecification of one over the other can lead to increased risk of Type I and Type II errors by generating spurious parameter estimates (Diamantopoulos, Riefler, \& Roth, 2008, p.1210). With classic reflective models (e.g., social attitudes, personality) the existence of the latent construct is independent of its measures. Formative models, by contrast, are "constructivist" in that the latent construct does not exist as an independent entity. Rather, formative models are wholly formed and defined by their indicators, which are presumed to be independent. Socioeconomic Status (SES) and the Human Development Index (HDI) are two well-established examples of formative constructs (Coltman, Devinney, Midgley, \& Venaik, 2008, p. 6) defined and caused by their underlying indicators. However, there is a unique nuance with formative models that involves the meaning of residual variance of the latent construct. 
In brief, the variance of a reflective construct is equal to the common variance of its indicators. That is, the variance of a formative construct incorporates the total variance of all of its underlying indicators - measured and unmeasured. However, unlike a reflective construct, a formative construct's disturbance term may represent "missing causes". Also, as the residual variance of the disturbance term increases "the meaning" of a formative construct "becomes more ambiguous" (Diamantopoulos et al., 2008, p.1212). Imposition of a reflective construction on a formative construct, via misspecification, deflates the true variance of the formative construct while increasing risk of "false-negative" findings (Type II error). Conversely, imposing a formative construction on a reflective construct may inflate the true variance of a reflective construct (Diamantopoulos et al., 2008, p.1210) leading to spurious findings of significance (Type I error). Another major difference between reflective and formative constructs is in the treatment of measurement error. Whereas the disturbance term $(\zeta)$ in a reflective model is associated with measurement error from the indicators, in formative models it is impossible to empirically assess measurement error or the meaning of measurement errors such as correlated errors (Coltman et al., 2007, p.11). Results from the four-factor EDA reflective analysis of the CES informed decisions on the CES items to be included in the construction of "testlet" factors. Consequently, the enumerated indicators of combat experiences form a potential constellation of antecedent causes of one's later combat experiences. Interestingly, unlike the indicators of a reflective model that would ideally exhibit the desirable property of high intercorrelations, formative indicators are not expected to share similar themes or 
preconceived patterns and may therefore exhibit no intercorrelation or high and low intercorrelations (Coltman et al., 2007, p.8).

The concept of "testlets" presented an additional modeling challenge as by definition testlets are dependent and bounded by a common stimulus similar to how response options to a test question are typically related to the question. As shown in Table 3., data were analyzed using Mplus (7.2) with 10,000 bootstrap draws to test three, four, and five testlet CFA solutions using 24 or 27 items with a Multiple Indicator Multiple Cause (MIMC) “2+ paths” to PTSS (i.e., ptsd3, ptsd4). Applying Hu and Bentler's (1999) combinatorial cutoff criteria rules [(.96 $\geq$ TLI and SRMR $\leq 0.09$; or RMSEA $\leq .06$ and SRMR $\leq 0.09$; or $.96 \geq \mathrm{TLI}$ and $\mathrm{SRMR} \leq 0.09$ )] for adequate indices of fit, results for the four formative testlets (i.e., 1.Combat; 2.Dead Bodies/Witnessing Death; 3. Proximate Engagement and 4. Close Calls) $\left[\left(X^{2}=19.365 ; d f=8 ; \mathrm{CFI} / \mathrm{TLI} \geq\right.\right.$ 0.977; 90\% RMSEA $(0.037,0.136) ; \operatorname{logLIK}\left(\mathrm{H}_{0}\right)=-2696.204 ; \mathrm{AIC}=5446.407$; $\mathrm{BIC}=5534.219 ; \mathrm{BICadj}=5448.693 ;$ SRMR=0.028; disturbance=0.014)] indicated a CES model (Figure 6.) with excellent fit, the lowest relative disturbance indices, and factor intercorrelations ranging from $r=.615-.746$. A more in-depth presentation of the analytic procedures to determine the best fitting CES model is available upon request.

Multiple regression analyses. Multiple regression models may be appropriate when a researcher believes the outcome of interest depends on more than one predictor variable. Additionally, multiple regression analyses may address how well predictors perform together as well as how much each predictor contributes to the dependent variable when controlling for the other predictors. Essentially, multiple regression 
analysis locates the two-dimensional plane or surface between the distributions of predicted and observed outcome values by finding a regression line that minimizes the ordinary least squared (OLS) distance of residual errors separating the predicted and observed values. When the predicted and observed values are highly correlated, the squared value of the multiple correlation coefficient (i.e., $R^{2}$ ) is an explanatory estimate of the amount of variance in the dependent variable (PTSS) that is accounted for by the independent variables (e.g., "CES," "ISO," and "SLEEP”).

However, if the regression plane of the multiple correlation coefficient amounts to "zero" (i.e., affirming the null hypotheses) the regression plane will be "flat" (e.g., Schmidt, 2011). This means that the values of the dependent variable are, on average, identical regardless of the level of the predictors. Lastly, multiple regression analyses provide an estimate of the magnitude of the predictive association (i.e., the slope of the regression coefficient) between each predictor and dependent variable on the scale of the dependent variable (e.g., Schmidt, 2011). Linear regression fundamentally assumes: (1) constructs are measured without error; (2) variables are normally distributed; and, (3) that significant predictors are linearly related to outcome variables (e.g., Schmidt, 2011) without exhibiting more complex relations (e.g., curvilinear or dose-response associations).

To investigate the second research question (Research Question\#2) of substantive interest, pertaining to whether the hypothesized effects differed by veteran employee group membership, or whether group membership (VES $x$ CES) moderated the effects of CES on PTSS, hierarchical multiple regression (HMR) and SEM analyses were used. 
The analytical frameworks of HMR and SEM were also applied to estimate whether iso-strain (ISO) and sleep quality (SLEEP) moderated the hypothesized main effect of CES on PTSS. HMR analyses provided an estimate of whether ISO and SLEEP demonstrated additional predictive power to explain variance in PTSS over and above the variance explained by CES and the five control variables of: (1) Socioeconomic Status (SES); (2) Time Since Last Deployment (DEPTIME); (3) Risk of Homelessness (HOME); (4) Conscientiousness (IPIPC) and (5) Neuroticism (IPIPN), all of which were included in each nested model.

Bootstrapping. As the univariate distribution of several focal variables (i.e., CES, PTSS) exhibited significant non-normality, nonparametric "bootstrap" methods, on the order of 10,000 randomized draws in Mplus (7.2), were utilized to calculate parameter estimates in the HMR analyses. "Bootstrapping" treats the sample data as a "population reservoir" by which random samples are drawn with "continuous replacement," so that the "probability of selection for any given case remains equal" (i.e., "selection probability remains $1 / N$ ) over every random draw (Kline, 2011, pp.42-43). In this manner, simulated bootstrapped samples "omit some members from the original sample" and include "other cases multiple times" (Kline, 2011, p.42-43). Theoretically, variability in the distribution of parameter estimates over many random bootstrapped samples accurately reproduces variability in the original sample (Kline, 2011). Moreover, with sufficient draws on a sufficiently large original sample, a simulated bootstrapped sample may accurately represent the variability from "the population from which (the original sample) was drawn” (Mallinckrodt, Abraham, Wei, \& Russell, 
2006, p. 373). To be clear, however, bootstrapping is not magic and the bootstrap method may still bias final parameter estimates when working with small samples $(N<80)$ that do not contain sufficient variability (Joyce, 2004)

Structural Equation Modeling. Structural equation modeling (SEM) is among the most firmly established and popular multivariate analytic methods in the social and behavioral sciences (Kline, 2011; Muthén \& Asparouhov, 2013, p.314). Typically, in SEM the relationship among observed variables (manifest) are formed by latent (unobserved) variables (Ullman, 2006) by way of linkage to common factors. The present thesis conceptualized two focal variables (CES, SES) as being represented by formative constructs defined by their respective observed variables. A unique advantage of SEM, especially for theory testing, is that since unreliability of measurement is calculated coefficients in theoretically critical areas of the model are thereby unaffected by measurement errors. Given the correct application of the SEM method and a representative, randomized probability sample greater cross-population stability of parameter estimates may be obtained than with such traditional methods as multiple regression or analysis of variance (Kline et al., 2011).

Typically, statistical software programs such as Mplus utilize "maximum likelihood (ML)" as the default estimator for SEM modeling. However, due to the extreme non-normality of the SERVe baseline sample data, and after several unsuccessful attempts at Box-Cox transformations of non-normal indicator variables, with greater model complexity both ML and MLR exhibited computational problems (e.g., non-convergence, non-positive definite latent "psi" covariance matrices) as 
indicated by Mplus error messages. Consequently, Mplus's "Bayesian random walk (RW)" estimator with "uninformed" and "informed priors" (e.g., from meta-analytic, population-level studies, expert opinion) may be integrated to estimate the hypothesized parameters of interest in SEM path analyses with and without underlying multipleindicators of the latent constructs. In addition to informative and uninformative priors, Bayesian analysis uses Markov chain Monte Carlo (MCMC) algorithms. According to the developers of Mplus (7.2), Bayesian estimators with uniform priors provide results "that would be the same as ML" estimation had the former been possible (Muthén \& Asparouhov, 2011, p.17). Additionally, better small-sample solutions and performance are possible as the Bayesian framework does not necessarily "require a large sample theory" (Muthén \& Asparouhov, 2011, p.17).

Model complexity. Model complexity and specification of the total parameters to be estimated is a fundamental principle in SEM. Model complexity refers to the lower diagonal form entries in the sample covariance matrix (Kline, 2011, p.101). These "observations" can be calculated via the formula " $v(v+1) / 2$ ", where " $v$ " equals the number of observed variables. For the proposed path analysis, the number of observations was 45, and for the possible multiple-indicator ("full-blown") SEM analysis the number of observations would be 351. A related limitation in SEM concerns "model degrees of freedom", where $d f_{\mathrm{M}}=p$ (observations)- $q$ (parameters). Ideally, model degrees of freedom must be $d f_{\mathrm{M}}>0$. For the path analysis, $d f_{\mathrm{M}}=45-9=$ 36 , and for the full-blown SEM model $d f_{\mathrm{M}}=351-26=325$ (Kline, 2011, pp.101-102). However, model complexity must also include whether the baseline sample size 
$(N=382)$ was sufficiently large enough to reliably estimate the two proposed SEM models. To answer this question, Jackson (2003) proposed the " $N: q$ rule"; whereby, the ideal the ratio of $N$ (sample size) to $q$ (parameters to be estimated) is 20:1. For the path model, $N: q \approx 42: 1$, and for the full-blown model $N: q \approx 15: 1$. According to the author, as $N: q<10$ the reliability of the results become compromised (Jackson, 2003)

\section{Results}

\section{Descriptive statistics, bivariate correlations, and prevalence rates}

As shown in Table 4., the means, standard deviations, and Pearson productmoment correlations for all untransformed variables in the study were provided. Combat Experiences (CES) were positively and significantly related to Sleep Quality (i.e., "PSQI-5" or "SLEEP") $[r=0.258, p<0.05]$ and PTSS $[r=0.407, p<0.05]$. In addition, Iso-strain (ISO) $[r=0.130, p<0.05]$ and SLEEP $[r=0.545, p<0.05]$ were positively and significantly related to PTSS. Among the control variables, SES $[r=$ $0.352, p<0.05]$ and Neuroticism $[r=0.517, p<0.05]$ were significantly related to PTSS. Although, SES was not related in the expected negative direction, suggesting that higher SES was not a protective factor in this sample. Interestingly, SLEEP $[r=0.258$, $p<0.05$ ] was significantly and positively related to CES. Also, Risk of Homelessness (HOME) was significantly and positively related to SLEEP $[r=0.284, p<0.05]$ and PTSS $[r=0.247, p<0.05]$. While CES and HOME $[r=0.076, p=n . s$.$] were unrelated,$ Conscientiousness and PTSS $[r=-0.128, p<0.05]$ were negatively related. As Sleep Quality and Iso-strain were expected to differ by veteran employee subgroup (VES), one-way ANOVA tests (Figures 8.-11.) were conducted on all focal variables. With the 
exception (Figure 9.) of PTSS $\left(F_{2 / 193.92}=3.388, p<.05\right)$, results from the ANOVA analyses were all nonsignificant (all $p$ 's $>.10$ ). The pattern of matrix correlations supported the main research hypotheses: CES, Iso-strain, and SLEEP were related to posttraumatic stress.

Baseline prevalence rates. Among SERVe's combat veteran employees $(N=382)$ self-reported nightly sleep duration $(M=6.06 / \mathrm{SD}=1.10)$ at baseline (Figure 11.) was, on average, 56.4 minutes less than the recommended nightly minimum of 7 hours for good health (Watson et al., 2015) with over 80\% (80.7\%) of SERVe combat veterans reporting less than 7 hours of sleep per night, on average, over the previous month and $17 \%$ reporting less than 5 hours of sleep, on average, per night. By using a recommended cutoff score of 12 (Figure 12., Bliese et al., 2008), 17\% of SERVe combat veterans met criteria for "probable PTSD" at baseline. Interestingly, this baseline rate of "probable PTSD" for a working sample of combat veterans fell between two other population-level estimates among post-9/11 veterans. Recently, in a "weighted prevalence study" of VA users and nonusers, Dursa and colleagues reported a 15.8\% rate of PTSD among deployed and nondeployed veterans (Dursa, Reinhard, Barth, \& Schneiderman, 2014); while in a meta-analyses Fulton et al. (2015) estimated prevalence of PTSD at $23 \%$.

\section{Hierarchical multiple regression analyses}

A hierarchal multiple linear regression was conducted to predict PTSS based on five predictors (i.e., CES, ISO, SLEEP, CES $x$ ISO, CES $x$ SLEEP) and five control variables (SES, HOME, DEPTIME, CONSCIENTIOUSNESS, NEUROTICISM). 
Using the forward selection procedure (Howell, 2013, p.542), five hierarchical multiple regression (HMR) analyses (Table 5. \& Table 5b.) with maximum likelihood estimation and 10,000 bootstrapped draws were conducted: one for each of the three proposed hypotheses, the second research question, and a fifth to test the total effect of CES, ISO, SLEEP, and VES along with their respective interaction term (CES $x$ ISO, CES $x$ SLEEP, CES $x$ VES) on the dependent variable (PTSS). In each of these analyses, after controlling for demographic (Socioeconomic Status, Risk of Homelessness), individual (Conscientiousness, Neuroticism), and military-related factors (Time Since Last Deployment), all mean-centered independent variables and interaction terms were entered in five steps. In Step 1, the five control factors were entered (centered at the grand mean) along with the main effect of CES. In Step 2, the mean-centered control factors were entered along with the mean-centered independent variables (CES, ISO) and the mean-centered two-way interaction CESxISO. In Step 3, the control factors were entered (centered at the grand mean) along with the prior mean-centered CES independent variable and the mean-centered independent SLEEP variable and the mean-centered two-way interaction CES $x$ SLEEP term. In Step 4, to test the total effect of the independent variables of CES, ISO, and SLEEP along with their respective twoway interaction terms (CES $x$ ISO, CES $x$ SLEEP), the control factors were entered (centered at the grand mean) along with mean-centered CES, ISO, and SLEEP independent variables and the mean-centered two-way interactions of CESxISO and CES $x$ SLEEP. In Step 5, the total effect of all the independent variables (CES, ISO, SLEEP, \& VES) along with their respective two-way interaction terms (CESxISO, 
CES $x$ SLEEP, CES $x$ VES), and the control factors were entered (centered at the grand mean) along with mean centered CES, ISO, and SLEEP.

As shown in Table 5. and 5b., applying an a priori exploratory significance level of $p<.10$, and after controlling for demographic, individual, and military-related factors in the nested series of hierarchical regression models, the hypothesized effects of the untransformed CES, ISO, and SLEEP variables were tested. Prior to hypotheses testing, the effect of all five control variables were investigated by regressing PTSS on the linear combination of SES, Time Since Deployment, Risk of Homelessness, and the personality variables. With the exception of the personality traits $(p>.10)$, all other control variables were significant predictors (all $p$ 's <.05), explaining $19 \%\left(R^{2}=0.198\right)$ of the variance in PTSS.

Hypothesis 1. Specifically, to test the first research hypotheses that levels of Posttraumatic Stress (PTSS) are a function of Combat Experiences (CES), a multiple regression analysis with 10,000 "bootstrap draws" was conducted. As shown in Table 5., results from the hierarchical multiple regression analysis provided support for the first research hypothesis (Model 1.). CES had a significant main effect $[\beta=0.368$ (0.049), $p<0.001]$ in the predicted positive direction on PTSS. Further, the baseline model (Model 1.) produced the following indicators of baseline model performance (Adjusted $R^{2}=0.331$ ). Overall, with the addition of the ISO variable $[\beta=-0.077$ (0.110), $p>.10]$ in Model 2., CES bordered on insignificance $[\beta=0.179(0.108), p=$ 0.098] as a predictor of PTSS and became insignificant in Models 3. through 4. Thus, as proposed, there was a positive main effect of CES on PTSS, which with the addition of 
the moderating variables of ISO and SLEEP became insignificant. Overall, research hypothesis\#1was supported. Results of the second research question (RQ\#2) are provided in the "Research Questions" section.

Hypothesis 2. To test the second research hypothesis on whether the interaction of ISO $x$ CES enhanced the main effect of CES on posttraumatic stress symptoms (PTSS) such that those scoring higher on ISO under the condition of high CES also scored higher, on average, on PTSS, a HMR analyses with 10,000 bootstrap draws was performed. As shown in Table 5., results from the HMR analyses (Model 2.) provided exploratory support for the enhancing effect of ISO on the association of CES and PTSS. In the ISO model (Model 2.) the interaction of ISO $x$ CES moderated the main effect of CES on PTSS in the predicted positive direction $[\beta=0.285(0.158), p=0.071]$. Inspection of a two-way interaction graph (Figure 13.) revealed that higher levels of ISO under the condition of high CES were associated with the highest levels of PTSS. Overall, the ISO model (Model 2.) produced the following indicators of model fit (Adjusted $R^{2}=0.352, R^{2} \Delta=0.021, p<0.01$ ). Thus, evidence from the HMR analysis supported the second research hypothesis: ISO enhanced the main effect of CES on PTSS in the predicted positive direction.

Hypothesis 3. To test the third research hypotheses on whether the interaction of CES $x$ SLEEP buffered the main effect of CES on PTSS such that those scoring lowest on SLEEP (i.e., good sleepers = lower Sleep Quality) under the condition of high CES also, on average, scored lowest on PTSS, a HMR analyses with 10,000 bootstrap draws was performed. Results of the HMR analyses (Table 5.) provided support for the 
buffering effect of good sleep (i.e., lower Sleep Quality) on the main effect of CES on PTSS. In the SLEEP model (Model 3.), the SLEEP $x$ CES interaction term failed to reach statistical or exploratory significance in the predicted positive direction $[\beta=0.197$ (0.205), $p>0.10]$. Interestingly, after controlling for individual, demographic and military-related factors, the main effect of SLEEP $[\beta=0.276(0.119), p<0.05]$ in Model 3. was significant as was the main effect of SLEEP $[\beta=0.268(0.113), p<0.05]$ in the combined ISO and SLEEP model (Model 4.). Overall, the SLEEP model (Model 3.) explained about $46 \%$ of the variance in PTSS (Adjusted $R^{2}=0.456, R^{2} \Delta=0.104, p$ $<0.05)$. Thus, evidence from the HMR analyses did not support the third research hypotheses: Sleep Quality did not buffer the main effect of Combat Experiences on PTSS.

Summary of hierarchical multiple regression. Overall, after controlling for demographic, individual, and military-related factors Combat Experiences, Iso-strain and Sleep Quality (Model 4.) explained about 47\% of the variance in PTSS (Adjusted $R^{2}=0.468$ ). In addition, after controlling for SES, risk of homelessness, personality, and time since last deployment in the full model (Model 5.), the main effect of Combat Experiences was insignificant $[\beta=0.156(0.208), p>0.10]$. However, in the combined model (Model 4.) neither Iso-strain $[\beta=0.221(0.151), p>0.10]$ nor Sleep Quality $[\beta=$ 0.189 (0.193), $p>0.10$ ] significantly moderated Combat Experiences on PTSS. Additionally, fit indices of the full model (Model 5.) indicated that inclusion of VES [ $\beta$ $=0.084(0.111), p>0.10]$ and the CESxVES interaction term $[\beta=-0.184(0.200), p>$ 0.10] were insignificant and failed to provide incremental prediction of PTSS (Adjusted 
$R^{2}=0.470, R^{2} \Delta=0.002, p>0.10$ ). Thus, the ISO and SLEEP model (Model 4.) was selected as the best overall fitting model (Adjusted $R^{2}=0.468, R^{2} \Delta=0.012, p<0.05$; $\left.\mathrm{AIC}=1967.173 ; \mathrm{BIC}_{\mathrm{adj}}=1976.444\right)$. In Model 4., participants' predicted PTSS was equal to:

$$
\begin{aligned}
& \hat{y}=7.997+0.004(\mathrm{CES})+-0.016(\text { ISO })+0.300(\mathrm{SLEEP})+.935(\text { ISOxCES })+0.938 \\
& (\mathrm{SLEEPxCES})+0.289(\mathrm{SES})+-0.011(\mathrm{DEPTIME})+1.802(\mathrm{HOME})+0.345(\mathrm{IPIPC})+- \\
& 1.325(\mathrm{IPIPN})+e
\end{aligned}
$$

Where, Risk of Homelessness (HOME) was coded as: " $1=$ Yes, 2 = No" and Time Since Deployment (DEPTIME) was measured in months since a participant's last deployment. According to the unstandardized beta weights from the equation for Model 4., a participant's PTSS increased 935 [PCLM unit of measure] for each ISOxCES unit of measure and 0.938 [PCLM unit of measure] for each SLEEPXCES unit of measure.

\section{Bayesian SEM with "Gibbs sampler" and "Random Walk" estimators}

Hypothesis testing and model fit. Tables 6. and 6b. provide a decomposition of the effects from the path analyses and shows that all five models: [Combat Experiences (Model 1.); Iso-strain (Model 2.); Sleep Quality (Model 3.); combined ISO and Sleep Quality (SLEEP) (Model 4.); and full ISO, SLEEP, and Veteran Employee Status (VES) (Model 5.)] exhibited excellent fit to the data as indicated by post-predictive $p$ values $(P P P$-val $\leq .01)$ that fell within $1 \%$ of the ideal cutoff value $(P P P$-val $=.50)$ for comparative "model checking" of Bayesian models (all PPP's $\geq .490$ ). These PPPvalues indicated that simulations with hypothesized models consistently replicated observed data to within 1\% of a discrepancy (Muthén \& Asparouhov, 2012). While lower $P P P$-values indicate greater model misfit, Bayesian one-sided $p$-values for 
parameter estimates in Mplus (7.2) have a default cutoff of " $p<.025$ " comparable to a two-tailed frequentist significance level of " $\alpha<.05$ " as indicated by asterisk (“*”) in the output. As a $p$-value of " $\alpha<.10$ " was selected a priori for "exploratory significance", in a Bayesian framework a comparable significance level for a one-tail test would require a $p$-value of " $p<.05$ ". Practically speaking, one-tail tests in Bayesian analyses are equivalent to the probability of the path coefficient being of the opposite sign and therefore insignificant (Muthén \& Asparouhov, 2012).

Bayesian SEM parameter estimates. As shown in Figure 15., the interaction paths in the ISO (Model 2.) and combined ISO and SLEEP model (Model 4.) were either exploratory ( $p<.05$, one-tail test) or conventionally significant $(p<.025$, one-tail test). In the combined model (Model 4.), while 95\% CIs of the standardized path coefficient for ISO $x$ CES included zero $(\beta=0.214,95 \%$ CI $[-0.054,0.479])$, the standardized path coefficient for the SLEEP $x$ CES term excluded zero $(\beta=0.394,95 \%$ CI $[0.088,0.693])$. Therefore, for Model 4., the interaction of ISOxCES reached exploratory significance $(p<.05$, one-tail test); while the probability of SLEEP $x$ CES interaction term was highly significant $(p<.01$, one-tail test). Again, statements of confidence in levels of statistical significance are based on a less than $1 \%$ likelihood of the standardized beta coefficient for the SLEEP $x$ CES term being of the opposite sign (Model 4.); while for the ISO $x$ CES term (Model 4.) there was less than a 6\% likelihood of the standardized beta coefficient being of the opposite sign.

For the main effects of ISO and SLEEP on PTSS (Model 4.), 95\% CIs for the direct path coefficients of ISO $[\beta=-0.059(0.102), p>0.10]$ and SLEEP $[\beta=0.168$ 
(0.088), $p<0.05]$ were, respectively, over and under exploratory significance. Similar to the HMR analyses, with the exception of the main effect of Conscientiousness ( $p$ >.10), results showed that all other control variables, including Neuroticism, were significant predictors (all $p$ 's $<.05)$, explaining $20 \%\left(R^{2}=0.201\right)$ of the variance in PTSS.

Reduced path models. Path analyses of the reduced ISO (Model 2.) and SLEEP (Model 3.) were inconsistent with the pattern of results from the combined ISO and SLEEP model (Model 4.). In the ISO model (Model 2.) the standardized path coefficient for the ISO $x$ CES term reached exploratory significance in the predicted positive direction $(\beta=0.281(0.151), p<0.05$, one-tail test). Whereas, in the SLEEP model (Model 3.) the path coefficient for SLEEP $x$ CES was insignificant $(\beta=0.194$ $(0.162), p>0.10$, one-tail test). Therefore, the probability of ISO $x$ CES moderating CES on PTSS (Model 2.) reached exploratory significance while the probability of SLEEP $x$ CES moderating CES on PTSS (Model 3.) was insignificant. Evidence from the path analyses for the reduced ISO (Model 2.) and SLEEP models (Model 3.) supported Hypothesis\#2 but failed to support Hypothesis\#3. In sum, ISO enhanced CES on PTSS and SLEEP did not buffer CES on PTSS in the reduced path models.

Model Selection. Among the five models the combined (Model 4.) ISO and SLEEP model: $[P P P$-val $=0.498 ; n f p=12 ; \mathrm{PSR}=1.000 ; \mathrm{DIC}=1962.004 ; \mathrm{BIC}=2009.504$; $\mathrm{pD}=11.898)]$ appeared to best replicate and therefore fit the data. Moreover, as shown in Model 2. and Model 4., applying the exploratory a priori $p$-value of " $\alpha<.10$ " for statistical significance suggests that ISO $(\beta=0.281(0.151), p<0.05$, one-tail test $)$ and 
$\operatorname{SLEEP}(\beta=0.394(0.154), p<0.01$, one-tail test $)$ moderated the direct path of CES on PTSS in the predicted positive directions. That is, greater ISO and poorer SLEEP under high CES predicted the highest symptoms of PTSS. However, in Model 4., while the direct path of SLEEP $[\beta=0.168(0.088), p<0.03]$ predicted increased PTSS; the direct path of ISO $[\beta=-0.059(0.102), p>0.10]$ did not. Finally, in Model 5., the interaction of veteran employee status (VES $x$ CES) with CES on PTSS was insignificant $[\beta=-$ $0.180(0.175), p>0.10]$. In Model 4., participants' predicted PTSS was equal to:

$$
\hat{y}=7.998+-0.038(\mathrm{CES})+-0.015(\text { ISO })+0.191 \text { (SLEEP) }+0.922 \text { (ISOxCES) }+2.354
$$$$
(\text { SLEEPxCES })+0.274(\text { SES })+-0.011 \text { (DEPTIME) + 1.745(HOME) + 0.370(IPIPC) + - }
$$$$
\text { 1.364(IPIPN) }+e
$$

Where, Risk of Homelessness (HOME) was coded as: " $1=$ Yes, 2 = No" and Time

Since Deployment (DEPTIME) was measured in months since a participant's last deployment. According to the equation of unstandardized beta weights from the Model 4., a participant's PTSS increased 0.922 [PCLM unit of measure] for each ISOxCES unit of measure and 2.354 [PCLM unit of measure] for each SLEEPXCES unit of measure.

Summary of HLR \& Bayesian Path Analysis. Theoretically, Bayesian path analysis with uninformed priors may produce results similar to SEM analysis with maximum likelihood estimation (MLE) had MLE been possible (Muthén \& Asparouhov, 2011). Results from the Bayesian path analysis did not replicate the pattern of results from the hierarchical multiple regression analyses. In all three HMR models (Models 3.-5.) where it was included, the effect of the hypothesized SLEEP $x$ CES interaction term was insignificant (all $p$ 's $s .10$ ). However, after applying a Bayesian methodology to better estimate population parameters from a range restricted 
sample with non-normally distributed variables, the interactions of ISO and SLEEP with CES in the path analyses (Model 4.) reached exploratory significance for ISO $(p<.10)$ and was highly significant for SLEEP $(\mathrm{p}<.01)$. The Bayesian analyses provided additional support that these associations may exist at the population level. In conclusion, evidence from both the HMR and BSEM supported the three research hypotheses: CES predicted changes in PTSS and ISO and SLEEP moderated CES on PTSS among this sample $(N=382)$ of working post-9/11 military veterans.

Power analyses. An a priori F-test power analysis (Figure 16.) based on five predictors for each model were calculated to achieve power $(1-\beta=.80)$ to detect a medium-sized effect $(F$-test $=.15)$. The results suggested that a sample of at least 94 participants in each veteran employee subgroup $(N=282)$ would be required to achieve recommended sufficient power $(1-\beta=.80)$. However, since the present study tested each moderation hypotheses (i.e., Hyp\#2 \& Hyp\#3) separately, before testing the moderating effect of ISO and SLEEP in a combined model, overall power to detect hypothesized effects was greatly improved. A post-hoc power analysis of HMR the ISO model (Model 2.) with 8 predictors (i.e., 5 control variables + CES, ISO, ISO $x$ CES) produced a large effect size $\left(f^{2}=0.366\right)$ with power $(1-\beta=1.000)$. Similarly, in the HMR analyses for the SLEEP model (Model 3.) with 8 predictors (i.e., 5 control variables + CES, SLEEP, SLEEP $x$ CES $)$ produced a large effect size $\left(f^{2}=0.326\right)$ with sufficient power $(1-\beta=1.000)$. Lastly, in the combined ISO and SLEEP model (BSEM Model 4.) produced a large effect size $\left(f^{2}=0.351\right)$ with sufficient power $(1-\beta=1.000)$. In summary, large effect sizes $\left(f^{2}>0.350\right)$ and a bigger sample $(N=382)$ than expected 
resulted in maximum power to detect effects for models in this study using multiple regression analyses.

\section{Research Questions}

In addition to the three research hypotheses, two interrelated research questions were proposed and analyzed. The rationale for the research questions addressed several primary concerns of the author. Research question number one $(R Q \# 1)$ was motivated by a desire to provide evidence of measurement invariance to lend greater confidence in any significant findings of group differences on two of this study's focal variables: "Combat Experiences" and "Posttraumatic Stress Symptoms". Essentially, the logic of measurement invariance dictates that if measures are invariant across identifiable groups, then greater confidence can be placed in any statistically significant group differences on the latent factors (Horn \& McArdle, 1992). A finding of measurement invariance would represent true group differences and not measurement artifacts caused by sample or error variance at the indicator and residual variance levels (Horn \& McArdle, 1992). Additionally, the results of an investigation of measurement invariance, even if unsuccessful, can provide clues on how to improve an underperforming measure.

Research Question \#1: Are measures of Combat Experiences and Posttraumatic Stress Symptoms invariant between identified veteran employee subgroups, and to what extent do results from tests of measurement invariance provide sufficient confidence in any significant findings of possible latent effects of stressors on strain?

Factorial invariance testing. Based on recommended procedures from Horn and McArdle (1992), measurement invariance tests of the Combat Experiences (CES) and PC-PTSD ("PTSS") scales were conducted in Mplus 7.2. Typically, in empirical and 
applied research, comparisons of means and regression coefficients are conducted on theoretically distinct groups from different populations. These analytic techniques assume that the underlying measures are equivalent across groups (Xu, 2012). To avoid this common oversight, the present study tested whether or not strong ("scalar") invariance in the linear associations between the latent constructs and intercepts of the measurement variables would produce regression weights ("loadings") and intercept terms that were invariant between three identified subgroups of veteran employees. However, before investigating the second research question, concerning proposed subgroup differences on the main effect of CES on PTSS, invariance testing among the three subgroups of veteran employees (i.e., Active Reservists, Separated Active Duty, and Separated Reservists) became a high priority.

Combat experiences and PTSS scales. Measurement invariance of the CES and PTSS scales among veteran employee subgroups is assumed if a multiple group measurement model achieves a level of strong factorial invariance of the factor loadings and intercepts (Model 3.), which exhibit significantly improved model fit. Specific changes in "goodness of fit" indices (i.e., $H_{0}: \Delta \mathrm{CFI} \leq-0.001$, Cheung \& Rensvold, 2002, p.251) provided a test of significance over the lesser constrained "weak invariance model" (Model 2.). Achieving a minimum of strong factorial invariance was a prerequisite to assessing any potential finding of significant group differences as implied by the second research question. As shown in Table 7., and according to Hu and Bentler's (1999) recommended combinatorial cutoff criteria rules [i.e., (.96 $\geq$ TLI and SRMR $\leq 0.09)$ ], the CES multigroup configural model (all factor 
loadings, intercepts, and residual variances freely estimated) failed to achieve adequate fit $[(T L I=.712 ;$ SRMR $=.101)]$. Therefore, further invariance testing was abandoned and it remains uncertain whether this misfit in configural invariance for the Combat Experiences Scale was due to: (1) subcultural differences in the meaning and frame of reference of the constructs among veteran employee subgroups; (2) data collection problems; (3) translation errors, or (4) other unknown factors (e.g., Cheung \& Rensvold, 2002, p. 237) such as small subgroup sample size (e.g., Active Reserve, $N=$ 69). Consequently, a true comparison of group mean differences on CES at the factor level cannot be assumed.

PC-PTSD Scale. Similarly, as shown in Table 8., measurement invariance testing for the PC-PTSD ("PTSS") scale also failed to achieve strong "scalar" invariance without significant decrements $\left(H_{0}: \Delta \mathrm{CFI} \leq-0.001\right)$ in goodness of fit indices. However, according to Hu and Bentler's (1999) combinatorial criteria rules [i.e., (.96 $\geq$ TLI and SRMR $\leq 0.09)$ ], the PTSS configural invariance model was an excellent fit to the data $[(T L I=.987 ;$ RMSEA=.079) $]$. Consequently, the null hypothesis of no difference between the strong invariance model and the configural model could not be rejected. In sum, support for $R Q \# 1$ was unfounded: Neither, the CES nor PTSS scales can be presumed to be invariant among self-identified subgroups of SERVe veteran employees.

Unlike the CES scale, however, it can be assumed that the three identifiable subgroups of SERVe combat veteran employees (i.e., active reservists, separated active duty, and separated reservists) conceptualized the construct of PTSS in similar ways. 
An inspection of the expected values of the intercepts across the four PCLM-PC items revealed that the Active Reserve subgroup was highest on item\#1 (i.e., "Repeated disturbing memories, thoughts, or images of the stressful experience" $[v=1.826$ (0.197), $p>0.05]$ ); item 2 (i.e., "Having physical reactions like heart pounding, trouble breathing, sweating when something reminded you of the stressful experience") $[v=$ $1.780(0.194), p>0.05]$ ); and item 4 (i.e., "Having difficulty concentrating" [ $\mathrm{v}=1.964$ (0.206), $p>0.05])$. In addition, the Separated Active Duty subgroup was highest on item\#3 (i.e., "Avoiding activities or situation, because they remind you of the stressful experience") $[v=1.690(0.115), p>0.05]$ while the Separated Active Reserve subgroup was lowest on all four PTSS items. Though the three PTSD factors (i.e., reexperiencing, avoidance, and intrusions/arousals) could not be modeled as latent constructs, due to the limited number of indicators per construct $(i<3)$, it should be noted that items 1 and 2 relate to the "reexperiencing" dimension of PTSD; while items 3 and 4, respectively, relate to the "avoidance" and "intrusions/arousals" dimensions (Bliese et al., 2008). Again, as scalar invariance was not achieved these findings are merely curious.

Research Question \#2. The second research question was motivated by a desire to test a bedrock assumption of job strain theory that greater levels of job stressors (e.g., work overload,) in the absence of sufficient levels of job control, decision latitude, social support, or other resources would predict relatively higher levels of strain (e.g., PTSS) among the most afflicted subgroups of veteran employees. To investigate the second research question on whether veteran employee status (i.e., active reserve, 
separated active, separated reserve) moderated CES on PTSS hierarchal multiple regression with bootstrapped standard errors (10,000 draws) and Bayesian path analyses were analyzed. As shown in Table 5b., results from the HMR $[\beta=-0.184(0.200), p>$ 0.10] and path analyses (Table 6b.) $[\beta=-0.180(0.175), p>0.10$, one-tail tests $]$ failed to support RQ\#2: veteran employee status (separated or active) did not moderate experiences of combat on PTSS. Moreover, results from $R Q \# 1$ make moot any potential findings of identifiable group differences on CES and PTSS among SERVe combat veterans until the sources of noninvariance with these measures are isolated and remedied. 


\section{Discussion}

After controlling for socioeconomic status, military, and personality related factors in addition to risk for homelessness, the results showed that modifiable organizational and individual factors affected severity and intensity of PTSD symptoms among threehundred and eighty-two working post-9/11 veterans living in the Pacific Northwest. Specifically, evidence from hierarchical multiple regression and Bayesian SEM path analyses provided exploratory support for the hypotheses that Iso-strain and Sleep Quality, respectively, enhanced and buffered combat experiences on symptoms of posttraumatic stress. Placing these findings into a larger theoretical context, however, is challenging. Currently, a paucity of theoretical research exists to inform future military reintegration studies, leaving $\mathrm{I}-\mathrm{O}$ researchers in this area to adopt an integrated approach. Historically, three major theoretical perspectives have informed models of disability and functional impairment: (1) biomedical; (2) social construction; and (3) biopsychosocial (Schultz, Chlebak, \& Stewart, 2016, p.3). The concepts and findings presented here align with the biopsychosocial tradition while also providing a basis for future Total Worker Health studies with combat veterans to isolate other specific intervention factors in the associations among CES, ISO, SLEEP and PTSS.

This thesis study's literature review and preliminary findings reveal that much more work is required to both replicate these exploratory results as well as to promote greater veteran well-being and the acceptance of veteran employees in the civilian workplace. For example, reviews by Kelloway and Barling (2010) and investigative news reports by Zoroya (2013) indicate that workplace interventions to increase support 
of veterans must be aimed at multiple levels. These levels include the supervision of veterans as well as changing organizational cultures that led to best practices to reduce the persistent entry barriers around veteran hiring stigma and employment discrimination dating to at least the Vietnam era. Further, empirical questions remain on whether support from supervisors, coworkers, or the combination of the two exert the larger effect on reducing veteran sleep quality, job strain, and PTSS. Additionally, the exploratory findings of significant interactions of Iso-strain and Sleep Quality with experiences of combat are consistent with prior organizational research (e.g., Demerouti et al., 2001; Halbesleben \& Buckley, 2004) on the effects of occupational stressors such as work overload on strain (e.g., exhaustion, burnout). Interestingly, both the HMR and BSEM analyses (Tables 6. \& 7.) found little support ( 9 of $10 p$ 's $>.05$ ) for the trait of conscientiousness (IPIP_3) as a predictor of PTSS. Some resiliency and positive psychology researchers (e.g., Stix, 2011) may consider this result to be an outlier due to the unreliability of measurement $(\alpha=.63)$ of conscientiousness in the SERVe baseline sample. Interestingly, recent research with post-9/11 veterans (e.g., Erbes et al., 2011) found that positive emotionality (PEM), of which conscientiousness may be a subfacet, was unrelated to PTSD and therefore failed to serve as a protective factor among military personnel. The remaining discussion will focus on implications for practice, scientific contributions, limitations, and future directions for organizational practitioners, researchers, and business leaders.

Implications for practice. These exploratory results provide additional evidence for encouraging organizations to review their current policies and practices aimed at 
reducing occupational stress and mental illness among veterans and other workers in high-risk occupations. In light of prior research showing that 25\% of PTSD cases follow a delayed onset trajectory, with combat exposure being one of three predictors (Smid et al., 2009), and work situations characterized by iso-strain predicting stressrelated illness (Mather et al., 2015) being replicated in the current study, the rationale for organizations to take a preventative approach with workers in iso-strain jobs appears to have little downside as the evidentiary basis for it grows. Below are four recommendations in ascending order of estimated capital investments for guiding organizational policies and practices:

1. Improving communication among workers and supervisors. One inexpensive avenue of improvement would be utilizing freely available interoffice communication/messaging software systems such as "Hip-Chat" to better connect and integrate organization-wide telecommuters with onsite staff and supervisors in one multitopic platform that is compatible with computers and smartphones. Portable interoffice software messaging systems facilitate rapid exchanges of important as well as trivial information across the comings and goings of the daily work cycle. Besides improving connectivity and communication among colleagues, departments, and work groups such an easy-to-implement technological upgrade may increase perceptions of social integration, embeddedness, acceptance, support, and control. All of which may promote stress-relief for newcomers during socialization into high-demand jobs, particularly iso-strain positions. In addition, ensuring a collegial, interoffice mechanism to request, without fear of retaliation, specific organizational and coworker assistance 
may be additionally beneficial by increasing perceptions of social support for isolated workers in high-risk and/or high demand jobs.

2. Compensation, wages, and job-relevant training. Another policy consideration would be a review of the pay scales and compensation packages of veteran workers to ensure that they are in line with non-veteran workers in comparable positions. Such a policy review of pay and compensation may decrease the historical tendency of organizations to inflict an initial veteran "wage penalty" (e.g., Teachman, 2004), which may take as long as a decade for veteran workers to financially recover from, that runs the added risk of triggering perceptions of an effort-reward imbalance predictive of stress-related disorders (e.g., Nieuwenhuijsen et al., 2010; Siegrist, 1996) like PTSD. More recent research by Vick and Fontanella (2017) among post-9/11 veterans (25 to 40 years old) revealed that veteran workers earned 3\% less than their non-veteran colleagues. Reasons identified for this pay gap included higher disability rates and lower education. To help reduce the educational differential, organizations should consider developing in-service trainings to improve the skills of veteran workers as well as flexible work schedules to allow their veteran employees to utilize their educational benefits, including distance education, to obtain job-relevant training and knowledge to improve their prospects of advancement or transition into higher-paying and/or less stressful jobs.

3. Increasing job control. For veterans and other workers reentering or adapting to the civilian workforce with physical disabilities and emerging psychological symptoms the role of modern assistive technologies (e.g., screen reading software, Braille 
displays, alternative devices to interact with computers) may enhance end-user experiences (e.g., Ruh, Spicer, \& Vaughan, 2009, p.69) for veterans with mild (e.g., tinnitus) to severe (e.g., blindness) disabilities while also increasing perceptions of job control, which is related to job strain and in the present thesis was linked to posttraumatic stress. Providing such adaptive technologies for workers with disabilities would also be in accordance with best practices guidelines (e.g., Ruh et al., 2009) as enumerated in the Americans with Disabilities Act (2008).

4. Promoting culture change. As more veterans of today's wars are returning with traumatic brain injuries and PTSD, which can impair attention, concentration, and memory, all of which may be a hindrance to organizational socialization and a hazard to operating equipment and machinery as well as increase feelings of detachment from others, it is important for coworkers, supervisors, and hiring managers to understand that such reactions to trauma are normal and that low social support is a known risk factor for PTSD (e.g., Brancu, Thompson, Beckham, Green, Calhoun, Elbogen et al., 2014; Olff et al., 2014). To avoid any perceptions of coercion or inauthentic social interactions, promoting such a message of targeted support for a subgroup of workers may benefit from delivery with tact. One way to accomplish this would be to have veteran workers participate in crafting forms and forums for communicating this message. Another way would be to intervene with training interventions informed by working veterans and their colleagues aimed at increasing coworker and supervisor social support at the organizational level while designing sophisticated content appropriate for veteran and non-veteran workers alike that includes actual veterans and 
other workers performing in and out of their designated work roles. One component of such training might include educating supervisors on the effects of shiftwork on sleep (e.g., Flo, Pallesen, Åkerstedt, Magerøy, Moen, Grønli, et al., 2013) and PTSD (Germain, 2013) and how work-related sleep loss may lower productivity, compromise safety, and how such policies as flex schedules and alternative scheduling may reduce worker fatigue (Krauss, Chen, DeArmond, \& Moorcroft, 2005).

However, this thesis's literature review would be remiss if it failed to comment on the professed societal goal of achieving greater veteran reintegration and retention in the workplace. The consistent findings from I-O psychology and other disciplines suggests that targeted trainings on building workplace support for veterans must go beyond educating the supervisors and coworkers of veterans and active reserve employees. Cultural diversity training addressing persistent hiring stigma and selection bias toward veterans (e.g., Overman et al., 2010; Stone \& Stone, 2015; Zoroya, 2013) will also be required. Such trainings must target human resource and hiring professionals who are the primary gatekeepers for entry into the U.S. workforce and civilian organizations due to their prominent roles in recruitment, candidate selection, and placement of prospective employees. As recommended by Ruh and colleagues (2009) such cultural diversity programs must include psychoeducational trainings on unlawful practices under the Americans with Disabilities Act (2008). Besides banning hiring discrimination based on disability, the ADA (2008) requires reasonable workplace accommodations for alternatively abled workers. In addition, federal laws such as the Uniformed Services Employment and Reemployment Rights Act (USERRA) regulate 
employment for active reservist employees, including such requirements as permitting at least 8 hours of turnaround between the end of weekend drill and the start of the civilian work shift. According to Noe and Ford (1992) only cultural diversity training can address persistent organizational barriers by targeting the values, stereotypes, and managerial practices of organizational leaders. Addressing workplace stigma and bias toward prospective veteran employees may be a prerequisite to greater veteran reintegration success in the modern workplace.

Limitations. In keeping with Cook and Campbell (1979) casual effects can only be said to exist if (a) there is covariation of the stressor with illness, (b) if the stressor manifested before development of illness, and (c) if plausible alternative explanations can be eliminated. Under such a strict rubric causal inferences can never be unequivocally demonstrated (e.g., Blalock, 1961; Dwyer, 1983; Holland, 1986). However, though causality can never be demonstrated, in applied research causality may be plausibly inferred in a longitudinal or experimental design so long as the stressor precedes the strain (e.g., temporal precedence), a probability sample from a representational sample frame is selected, and confounding variables are ruled out (Zapf et al., 1996, p.147). Though evidence derived from this study's quantitative analyses provided support for each of the three research hypotheses several concerns relevant to research design, cross-sectional statistical modeling, and sample constraints limited the generalizablity of the results.

Sample limitations. As only baseline, cross-sectional, self-reported panel data from a sample of veterans living and working in the Pacific Northwest were analyzed, this 
study's results must be viewed with caution and considered to be preliminary.

Theoretically, cross-sectional data from a non-probability sample of U.S. post-9/11 veterans lacks sufficient temporal precedence and demographic diversity (Figure 17.) to draw causal inferences and generalizable claims from any significant effects (e.g., Brewer, 2000). In addition, the potential for range restriction, where the associations between variables can be reduced by limits on the range of values in one or more focal variables, was high due to the parent study sampling from only a portion (i.e., employed veterans) of the overall post-9/11 veteran population of interest. Typically, better estimates of the associations among variables are obtained from samples representing the population of interest (Cohen \& Cohen, 2003).

Retrospective measurement. Also, as measures of the personality (mini-IPIP), combat experiences (CES), and PTSS (PC-PCLM) phenomena were all taken retrospectively, there is the potential confounding of personality with trauma as has been cautioned by prominent trauma researches who have studied post-9/11 veterans. Briefly, Bonanno (2012) cautioned against contemporaneous measurement of personality and trauma, because of the potential for trauma to alter personality. Similarly, there is the potential confounding of neuroticism with the association of CES and PTSS, which may have reduced the variance in PTSS when controlling for personality. As shown in Table 4., PTSS and neuroticism were significantly and positively related $(r=0.517, p<.05)$ suggesting that greater levels of neuroticism are a risk factor (e.g., Cohen \& Edwards, 1989) for higher levels of PTSS. However, other research (e.g., Watson \& Casillas, 2003) has found that levels of trait neuroticism may 
be a defense mechanism that provides situational adaptability. Though meta-analytic findings (Brewin, Andrews, \& Valentine, 2000) informed five control factors for these thesis analyses, at least 10 other variables: gender, age, intelligence, race, psychiatric/traumatic histories (e.g., depression, TBI), childhood abuse/adversity, history of family mental illness, and general life stress are also potential risk factors for PTSD. To this list, several military-related factors (e.g., unit cohesion and support, supportive military leadership) are also related to PTSD (e.g., Bliese \& Castro, 2000; Pietrzak, Johnson, Goldstein, Malley, Rivers, Morgan, \& Southwick, 2010). Therefore, anyone or more of these uncontrolled factors may have influenced the results and threatened internal validity. Additionally, other limitations of this study concern identifying, excluding, and controlling for other common comorbid mental health conditions (e.g., anxiety and depression) potentially related to PTSS. As SERVe is an organizational study of veteran workers, prescreening for preexisting mental health comorbidities, psychotropic medications, tobacco, and alcohol use was not a deemed a priority. Failure to control for usage of psychotropic medications, for example, may have confounded the association of sleep with PTSS.

Psychometric concerns. Psychometric adaptation of the Iso-strain scale (Mathers et al., 2015), by adding the "Schedule Control" dimension, was less of a concern than the underperformance of such control measures as Conscientiousness $(\alpha=.63)$, which requires further investigation. Inspection of a scatterplot (Figure 18.) comparing Mather et al.'s (2015) Iso-strain composite measure (X-axis) with this thesis's adapted Isostrain composite measure ( $Y$-axis) revealed a significant positive correlation $(r=.71$, 
$p<0.01)$ indicative of two measures exhibiting convergent validity. Also, as it is unknown whether the association of sleep difficulties and PTSS was due to other psychological (e.g., depression) or medical conditions (e.g., asthma), including prescription and nonprescription drug use, the significance of this finding warrants some skepticism. More specifically, research with non-clinical samples suggests the PSQI may be a proxy measure of depression (Grandner, Kripke, Yoon, \& Youngstedt, 2006). Additionally, exploratory analysis (Figure 6.) indicated that the CES was best represented as a formative construct instead of a reflective one. That is, CES may be more conceptually meaningful when its indicators are modeled to cause it latent construct instead of the latent construct presuming to cause its indicators. Among the challenges of measuring PTSS, military researchers (e.g., Dohrenwend et al., 2007; Shea, Reddy, Tyrka, \& Sevin, 2013) have criticized the unreliability of self-report measures of posttraumatic stress on the basis of systematic exaggeration or underreporting of traumatic exposures, associated symptoms, and the absence of trained clinical judgment to differentiate symptoms of other co-morbid disorders such as depression (e.g., Gros, Price, Magruder, \& Frueh, 2012). Another related measurement issue is the lack of theory to inform predictions of how PTSD symptom clusters vary over time and may interact with changing individual and environmental circumstances. Lastly, self-reported measurement of PTSD relied on three of four components (i.e., reexperiencing, avoidance, and hyperarousal) that excluded the most recent additional clinical dimension of "negative alterations in cognitions and mood" (Criterion D, APA, 2013, p.271). Only one prior study (Shea et al., 2010) found that "avoidance/numbing" 
and "hyperarousal" to be predictive of occupational functioning (among a sample of National Guard and Reserve veterans). In the final analysis, these three symptom clusters of PTSD may not be as psychologically relevant among a selective sample of presumably high functioning and gainfully employed veterans several years postreintegration. There is also reason to be concerned with the Iso-strain measure being formulated as a relative composite of job demands, decision authority, schedule control, and social support at work. As each dimension was subtracted from the other, iso-strain was computed on a relative continuous scale without calibration of any magnitude of differences arising from equal intervals.

Potential for multilevel homogeneity of error variance. Lastly, the possibility for hierarchical contextual (“design effects”) are highly probable with these data. That is, SERVe's randomized-cluster waitlist-control design may have produced a homogeneity of variance violating the assumption of the independence of scores by way of veterans nested within higher-order participating organizations, work teams, or their occupational roles (Kline, 2011, pp.343-347). Therefore, future studies should explore multilevel analyses with particular attention on accounting for potential variation in job stress by occupation. Ergo, because of these enumerated design and measurement limitations the proposed study contained low internal and external validity. Nevertheless, given the rich cross-sectional data set and apparent absence in the reintegration literature of studies exploring potential links among civilian job strain, symptoms of insomnia and PTSD and how associations among these common reintegration factors may differ between subgroups of veterans, the rationale to conduct 
the proposed thesis study in service of expanding scientific knowledge of phenomena critical to military reintegration following combat appeared urgent.

Contributions. An overarching aim of the current thesis was to propose "isostrain" as a candidate variable for extending the nomological network of military-tocivilian reintegration into the civilian work domain. The finding of iso-strain and sleep quality interacting with combat experiences on PTSS was consistent with prior occupational health theories (e.g., COR, JD-R) and now requires the working out of the relative sequencing of these variables in causal models that may test potential reciprocal effects over multiple time points. A longitudinal design would allow for testing specific reciprocal effects from stressors driving higher symptoms of PTSS that in turn may drive higher levels of stressors and consequently higher PTSS from resource loss spirals and the prolonged activation of the JD-R's health impairment process.

Taken together, these exploratory findings contribute to existing scientific knowledge on military reintegration into the work and family domains (i.e., sleep is presumed to occur in the domestic sphere). Though preliminary, these results may also contribute to organizational practices with workers in high-risk occupations (e.g., prison workers, first responders) who already face high levels of daily hassles and life stress as job strain, insomnia and PTSD are all associated with negative health outcomes (e.g., heart disease, diabetes, dementia, shortened lifespan) and important organizational outcomes such as job satisfaction and intentions to quit (e.g., Karasek \& Theorell, 1990; Mysliwiec et al. 2013). Additionally, understanding factors tapping individual differences such as insomnia and personality traits, which may be relevant to predicting 
risk and resilience for PTSD among post-9/11 military veterans, may also help to "broaden the criterion space" and better inform an ultimate criterion (Thorndike, 1949) for how veteran reintegration into the workplace -along such dimensions as personnel selection, organizational retention, turnover, absenteeism, and intentions to quit - are measured and assessed in the future. By exploring the potential effects of the postdeployment civilian work environment on symptoms of PTSS among recently reintegrated veterans, findings from this thesis may contribute to informing development of evidence-based, recovery-oriented interventions at the organizational level as well as customizing current trainings designed to increase workplace support for reintegrating military personnel and the understudied Reserve Component.

Methodological contributions. Theoretically, by better approximating the uncorrected nonnormality distribution of several positively skewed focal variables (i.e., CES, PTSS), the Bayesian SEM framework appeared to have successfully improved upon the findings from the HMR analyses by "shrinking"viii standard errors toward zero. For example, under the multiple regression framework using "bootstrapping" standard error estimates for the CES $x$ ISO moderator $[\beta=0.285(\mathbf{0 . 1 5 8}), p=0.071]$ and CES $x$ SLEEP moderator $[\beta=0.197(\mathbf{0 . 2 0 5}), p>0.10]$ were larger than under the Bayesian framework: CESxISO $[\beta=0.281(\boldsymbol{0 . 1 5 1}), p<0.05$, one-tail test $]$ and CES $x$ SLEEP $[\beta=0.394(\mathbf{0 . 1 5 4}), \mathrm{p}<0.01$, one-tail test $]$. These comparative results (MLE vs. Bayesian uninformed priors) differed more than predicted in Muthén and Asparouhov's treatise (2011, p.17) on BSEM. In addition, the Bayesian models provide evidence supporting the utility of alternative quantitative methods to improve upon 
traditionally problematic sample limitations that may fail to detect subtle effects with high dimensional data (Mallick \& Yi, 2013). Applying an integrated frequentist and Bayesian framework in a random control design may help advance the state of organizational science regarding the development of methods to tests previously unexplored relationships among novel variables nested within complex data sets.

\section{Future directions \& Societal Relevance}

After conducting twenty years of longitudinal research on Israeli combat soldiers, Solomon and colleagues (2009) recommended that trauma researchers need to "put more emphasis" on studying "specific symptoms clusters" and stop treating PTSD "as a unified, undifferentiated disorder" (Solomon et al., 2009, p.843). Their recommendation for the future of PTSD is particularly germane to the holistic vision of "Total Worker Health" goals. Among these goals is the identification of risk factors related to workplace health problems (National Institute of Occupational Health [NIOSH], 2016). Findings from the present thesis study, the proposed theoretical model, and applied quantitative methods provide a flexible analytical framework and program of future research aimed at generating new scientific knowledge and a better understanding of military-to-work reintegration and veteran worker health and well-being.

Since the process of how the work-related experiences of veterans may influence their long-term mental health and economic stability remains both a long-neglected issue as well as theoretically lacking, the goal of future veteran work reintegration research should be aimed at improving veteran success, stability, and economic opportunities in the civilian work domain in addition to testing the boundaries of the 
nomological network of military-to-civilian reintegration with both established (e.g., sleep quality) and less well-understood variables and veteran workers (e.g., iso-strain, role ambiguity, amputees with and without PTSD) cross-validated on representational probability samples of post-9/11 veterans, earlier eras, and other emerging veteran subpopulations (e.g., female veterans, working homeless veterans) to inform theoretical development and customize ongoing intervention work.

Known unknowns. Little to no research has identified what specific workplace social supports and individual dispositional traits best buffer or enhance specific clusters of symptoms related to polytrauma and the PTSD syndrome. With regard to this research gap, the present study contributed to a preliminary understanding of how and why the proposed buffers and enhancers of sleep quality and iso-strain following combat may moderate three core dimensions of PTSD. Most importantly, in addition to knowing what modifiable organizational factors may foster greater veteran retention and high performance in the workforce there is an empirical need to understand the multi-faceted phenomena driving working-age veterans to drop out of the labor force and cease engaging in job-seeking behaviors (U.S. Department of Labor, 2015). Exploration of this missing piece of the work reintegration process may help to inform new approaches to keeping more veterans gainfully employed in a future labor force looming with a raft of returning veterans and their uncharted reintegration issues (e.g., Taber \& Hurley, 2009; Tanielian, 2009; Mysliwiec, Gill, Lee, Baxter, Pierce, Barr, Krakow, \& Roth, 2013). Additionally, research into the role of changing social identity and other nonpathological cognitive-affective factors among reintegrating veterans and 
the connection between their individual professional needs and their personenvironment fit (e.g., Greguras, 2009; Griffith, 2009) in various industries and organizations, public and private, may provide a deeper understanding of how the organizational socialization process varies for different veteran workers. Practical concerns of research design and the focus of interventions require attention, too.

Future research topics and designs. Despite the wars since 9/11 entering their sixteenth year, organizational and occupational health scholars have yet to publish any pre-to-post-deployment multilevel longitudinal intervention studies among veteran workers within the organizations that employ them. For example, investigating whether symptoms of insomnia at Time 1 for workers in differing work unit sizes and occupations predict intrusions/arousals at Time 2 and avoidance behaviors and supervisor performance ratings at Time 3 would be a relatively straightforward research design with enormous theoretical and practical consequences for improving how organizations can better support returning veterans to maximize their job security and financial stability. Also, there is a gap in research on whether the organizational context (e.g., abusive supervision) exacerbates prodromal symptoms (i.e., nightmares, foreshortened future) of PTSS with newly reintegrated veteran employees and other workers returning to high-risk occupations and how these contextual factors interact with prodromal symptoms to predict the burnout process (e.g., Halbesleben \& Buckley, 2004; Maslach, 2003; Vinokur, Pierce, \& Lewandowski-Romps, 2009) and onset of PTSD. A finer-grained goal of future research would be to more thoroughly explore how different clusters of PTSD and other psychological states (e.g., depression, anger, 
happiness), following experiences of combat, vary over time under different job situations (e.g., iso-strain, active jobs, passive jobs) and are impacted by such organizational justice variables as equity and fairness.

In addition, future research should explore whether or not positive psychological interventions (e.g., employee assistance plans, job rotation, charitable fundraising challenges) that are intended to increase available resources, build work team cohesion, and foster greater workplace integration and social capital attenuate the cumulative impacts of such known stressors and occupational impairments among veterans as combat experiences, TBI, and PTSD and affect work engagement as predicted by the JD-R (Bakker \& Demerouti, 2007). For example, a repeat-measure, longitudinal design could test whether a randomized waitlist control "team building program" acts as a buffer against specific facets of PTSD (e.g., reexperiencing, hyperarousal, avoidance/numbing, changes in cognition and mood) at different time points and under different work conditions (e.g., high vs. low job strain, supervisor support, newcomer status vs. tenured). Further, such an intervention study could be extended to investigate whether different clusters of PTSS are in turn these related to dimensions of iso-strain (e.g., demands, control, support) at later time points and how these intervening variables may predict indicators of well-being such as sleep quality (e.g., duration, latency, sleep efficiency). Such a research design would test the boundaries of the current work reintegration nomological network. Also, as research into onset of PTSD suggests rapid onset post-deployment, future work-family reintegration studies should measure a sample throughout the entire deployment cycle to better capture how the pre-to-post 
reintegration process unfolds to influence specific prodromal symptoms (e.g., nightmares) of PTSD and predictors of delayed onset (i.e., combat exposure, Western heritage, moral injury) and how these in turn are differentially related to organizational variables and outcomes such as personnel selection, work performance, wage premiums, advancement, and intentions to quit. There is also a need to better understand the influences from nonwork life and social networks (e.g., Berkman \& Syme, 1979) outside of work (e.g., friends, family, peers) affect PTSD. In particular, a greater understanding is needed of how the family domain (e.g., Ertel, Koenen, \& Berkman, 2008) may spillover into the work domain (i.e., family-to-work interference) to alter the course of PTSD, the work situation (e.g., iso-strain) and organizational outcomes (e.g., job performance, intentions to quit) and whether supervisor support or family-supportive workplaces (e.g., Odle-Dusseau, Herleman, Britt, Moore, Castro, \& McGurk, 2013) moderate these associations. A replication of the findings from the current study in a longitudinal design would also allow for further investigation of a potential curvilinear or dose-response associations among combat experiences, isostrain, sleep and PTSS and a potential polynomial effect of $\mathrm{CES}^{2}$ on PTSS.

Recruitment, analytical methods, optimization. Future research must recruit large enough samples to maximize the theoretical advantageous of using a structural equation modeling (SEM) approach to control for correlated errors and measurement error variance, while being flexible enough to modify a planned analysis in the face of underrecruitment such as by applying Bayesian quantitative methods. Along these lines, research variables must be selected to optimize research opportunities and provide 
practical advice to industry as they arise. For example, if well-designed longitudinal studies reveal that iso-strain predicts sleep quality, or even vice-versa, then organizations could maximize the effect of limited intervention resources by targeting one or the other with the practical expectation of achieving improved outcomes on both. From a prevention perspective, understanding the sequencing and associations among these variables is critical to understanding whether iso-strain and sleep quality are robust risk factors, moderators, or mediators of PTSS and upon which variable it is most important to intervene upon.

Societal relevance. Fully resolving the question of by what process does the civilian work milieu shape PTSD will require identifying what dimensions of the work reintegration experience to study? In closing, this thesis study has started to unravel this critical question by providing future investigators and the present author with a longterm plan of research for developing a more scientific understanding of the military-tocivilian reintegration process. I anticipate sharing any significant findings from this thesis and future discoveries that may be relevant to intervention work targeting the recruitment, selection, retention, job satisfaction, health, well-being, and economic success of the million working-age veterans projected to enter the U.S. workforce by 2019. Thereby, by doing so, the ultimate aim of presenting the results from this thesis study is to enhance the quality of life of future veterans both with their families and within society. 


\section{Endnotes}

${ }^{1}$ According to Joshi (2013) 'high dimensional data' is data with multiple dimensions, multiple variables, or multiple attributes.

${ }^{2}$ Stereotypes are the result of associations between attributes (e.g., personality traits, behaviors) and social categories (e.g., gender, age groups, occupations, race). The role of stereotypes in the workplace is relevant because stereotypes can influence work decisions. Indeed, decision-makers with unfavorable stereotypes about a group make unfavorable attributions about group members (Hewstone \& Jaspers, 1982, as cited by Bertolino, Truxillo, \& Fraccaroli, 2011, p.868).

${ }^{3}$ The three new symptoms of PTSD that were added in DSM-V (2013) are: "persistent and exaggerated negative beliefs about oneself, others, or the world; persistent distorted cognitions about the cause or consequences of the trauma; and reckless or self-destructive behavior".

${ }^{4}$ Focal and auxiliary variables used for Bayesian multiple imputation in Mplus (7.2) were: IMPUTE= ces01-ces27 deptimesmonths IPIPC IPIPN ipip1 ipip2R ipip3; IMPUTE= ipip4R ipip5 ipip6R ipip7 ipip8R milrank(c)

${ }^{5}$ One 55-year-old participant endorsed only CES item\#3 (i.e., "Seeing dead bodies or human remains").

${ }^{6}$ In brief, that compared classic frequentist estimation of focal parameters with Bayesian methods using optimal resampling algorithms.

${ }^{7}$ The three new symptoms of PTSD that were added in DSM-V (2013) are: "persistent and exaggerated negative beliefs about oneself, others, or the world; persistent distorted cognitions about the cause or consequences of the trauma; and reckless or self-destructive behavior".

${ }^{8}$ In brief, that compared classic frequentist estimation of focal parameters with Bayesian methods using optimal resampling algorithms.

9 "Shrinkage" is where extreme values in a sample are "shrunk" towards a central value, like the sample mean, or toward zero. 
Figure 1. Proposed Conceptual Model: Combat Experiences (CES), Veteran Employee Status (VES), Iso-strain, \& Sleep Quality moderate symptoms of posttraumatic stress (PTSS)

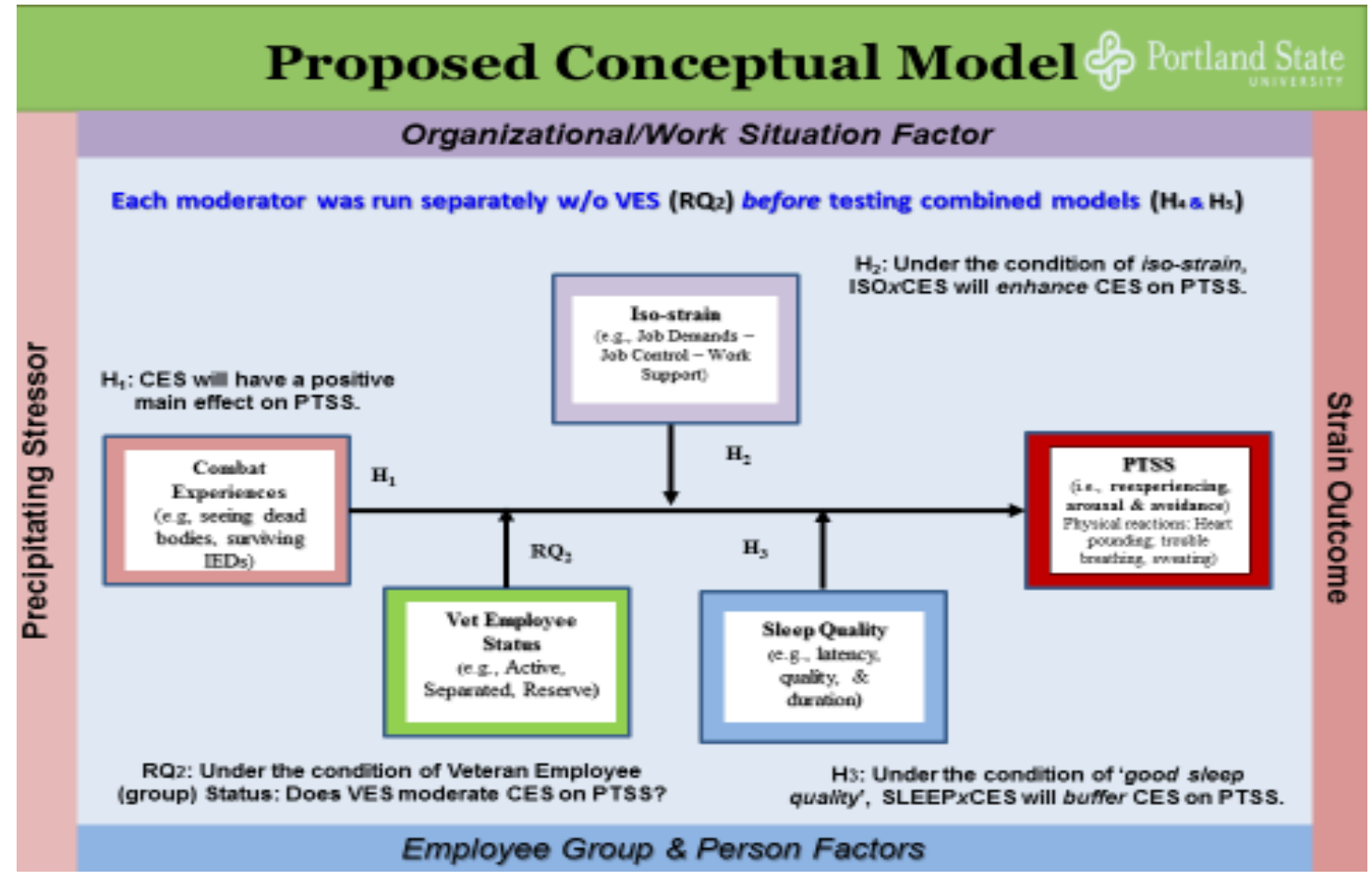


Figure 2. Changes from DSM-IV to DSM-5: Clinical diagnostic criteria for PTSD

\section{PTSD: DSM-IV vs. DSM-5}

\section{DSM-IV}

\section{A: stressor: need 2 of 2:}

1) experienced, witnessed, or was confronted with traumatic event and 2) intense fear, helplessness, or horror.

B. traumatic reexperienced: need 1 of S:

(1) Recurrent and intrusive distressing recollections:

(2) distressing dreams; (3) flashbacks; 4) intense psychological distress at exposure to cues; (5)

Physiological reactivity on exposure to cues

C. persistent avoidance of stimuli associated with the trauma and numbing: need 3 of 7 :

(1) Efforts to avoid thoughts, feelings; (2) Efforts to avoid activities, places, or people; (3) Inability to recall an important aspect of the trauma; (4) Markedly diminished interest; (5) Feeling of detachment /estrangement; (6) Restricted affect; (7) Sense of a foreshortened future

D. persistent increased arousal: need 2 of 5 : (1) Difficulty falling/staying asleep; (2) Irritability/ outbursts of anger; (3) Difficulty concentrating; (4) Hypervigilance; (5) Exaggerated startle response
DSM-5

A: stressor: need 1 of 4:

1) Direct exposure; 2) Witnessing; 3) Indirectly, by learning a close relative or close friend was exposed; 4) Repeated/extreme indirect exposure in the course of professional job (not through media).

B: intrusion symptoms: need 1 of 5 :

1) Recurrent, intrusive memories; 2) Traumatic nightmares; 3) flashbacks; 4) intense/prolonged distress after exposure; 5) physiologic reactivity upon exposure to cues

C: persistent effortful awoidance of distressing trauma-related stimuli: need 1 of 2 :

1) Trauma-related thoughts /feelings; 2) Traumafelated external reminders

D: nepative cognitions/mood: need 2 of 7 :

1) Inability to recall key features of the trauma; 2) negative beliefs about oneself, the world; 3) distorted blame of self, others; 4) Persistent negative traumafelated emotions; 5) diminished interest; 6) Feeling alienated, detachment/estrangement; 7) Constricted affect

E: alterations in arousal and reactivity: need 2 of 6 : 1) Irritable or aggressive behavior; 2) Self destructive/ reckless behavior; 3) Hypervigilance; 4) Exaggerated startle response; 5) Problems in concentration; 6) Sleep disturbance. 
Figure 3. Predictions from the Job Demands Resources model based on interaction effects

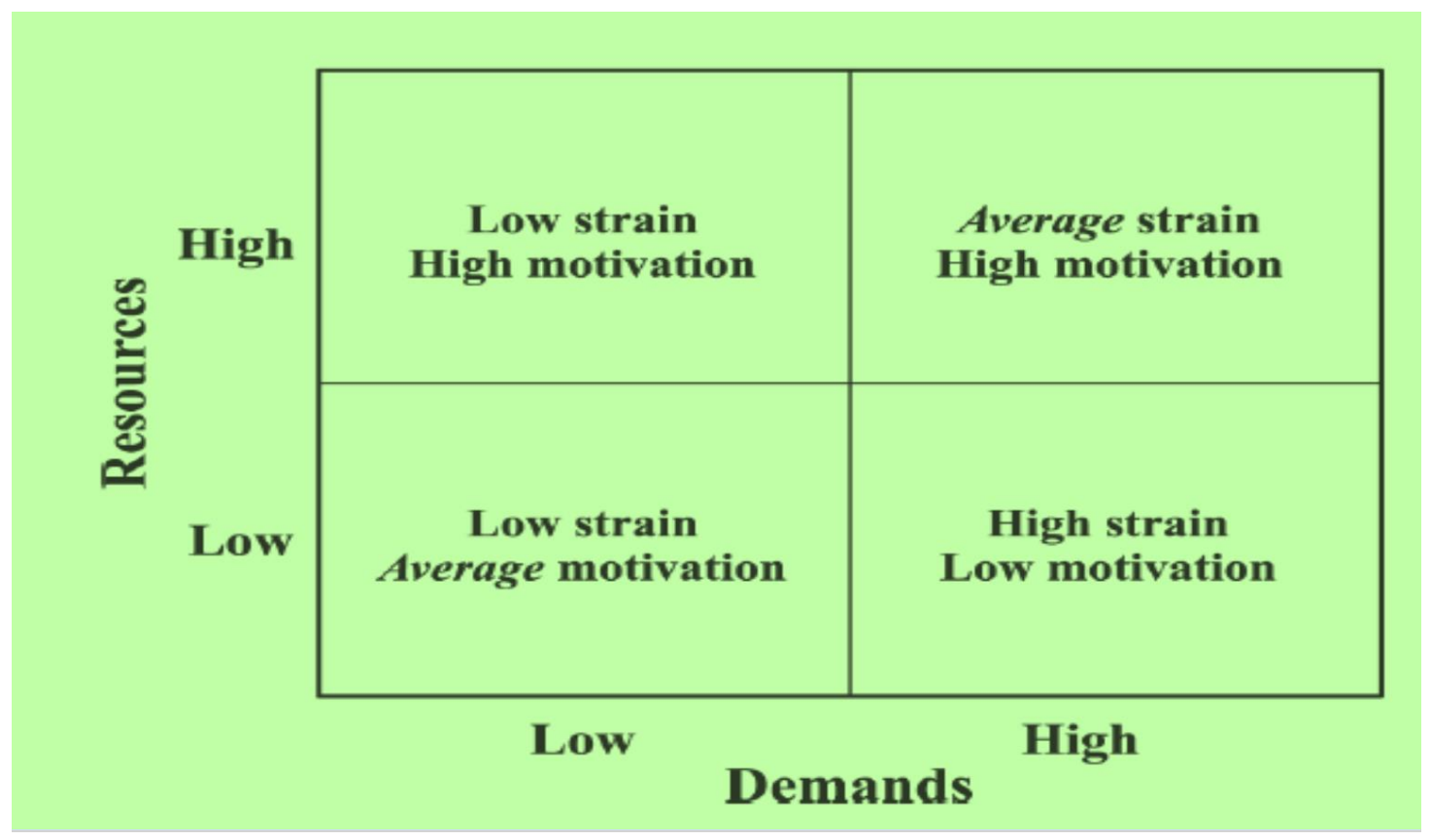

-- Bakker \& Demerouti (2007) 


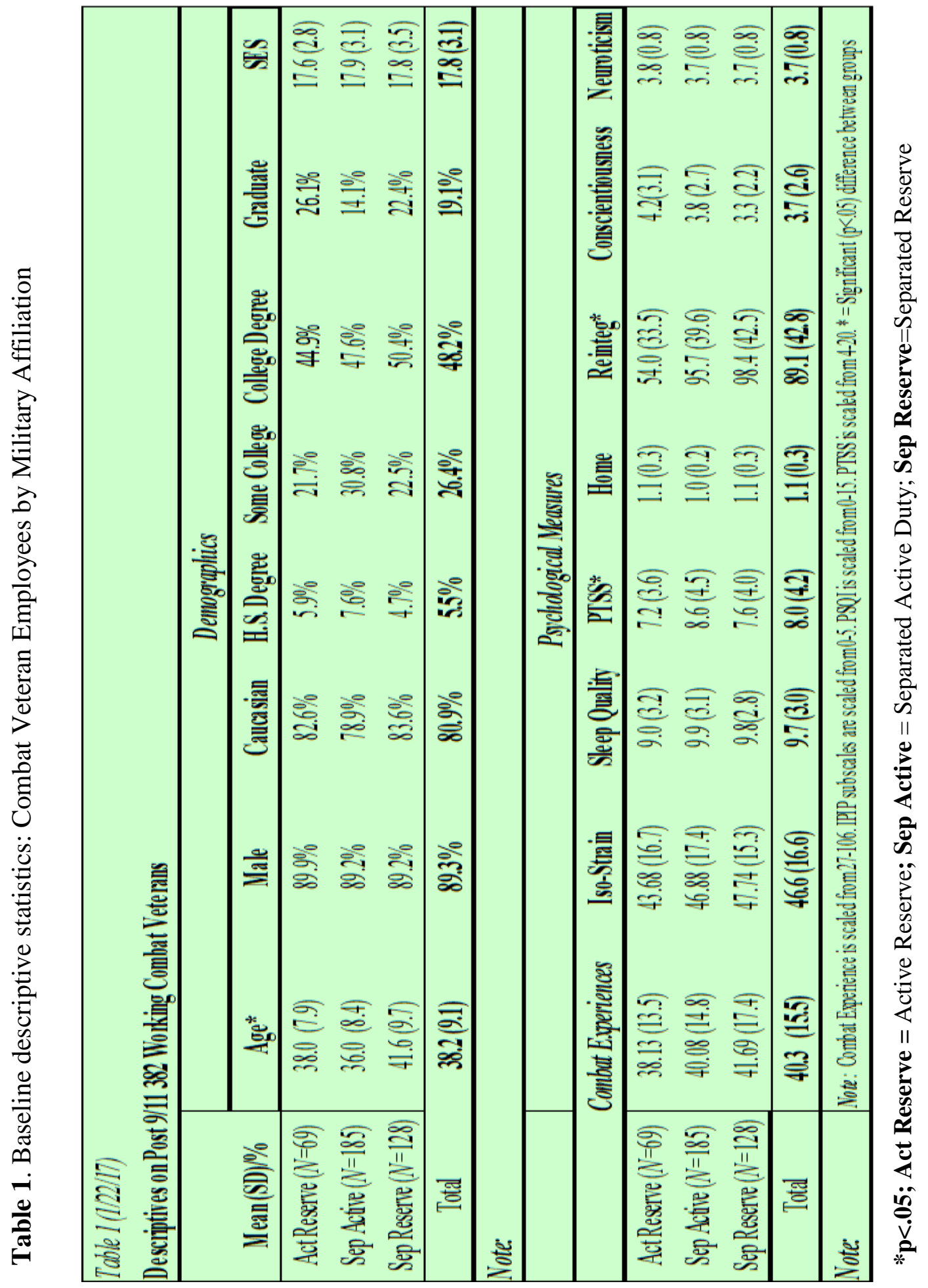


Figure 4. Hypothesis \#2: Iso-strain will moderate Combat Experiences on PTSS

\section{Proposed Enhancing effect of CESxISO on PTSS}

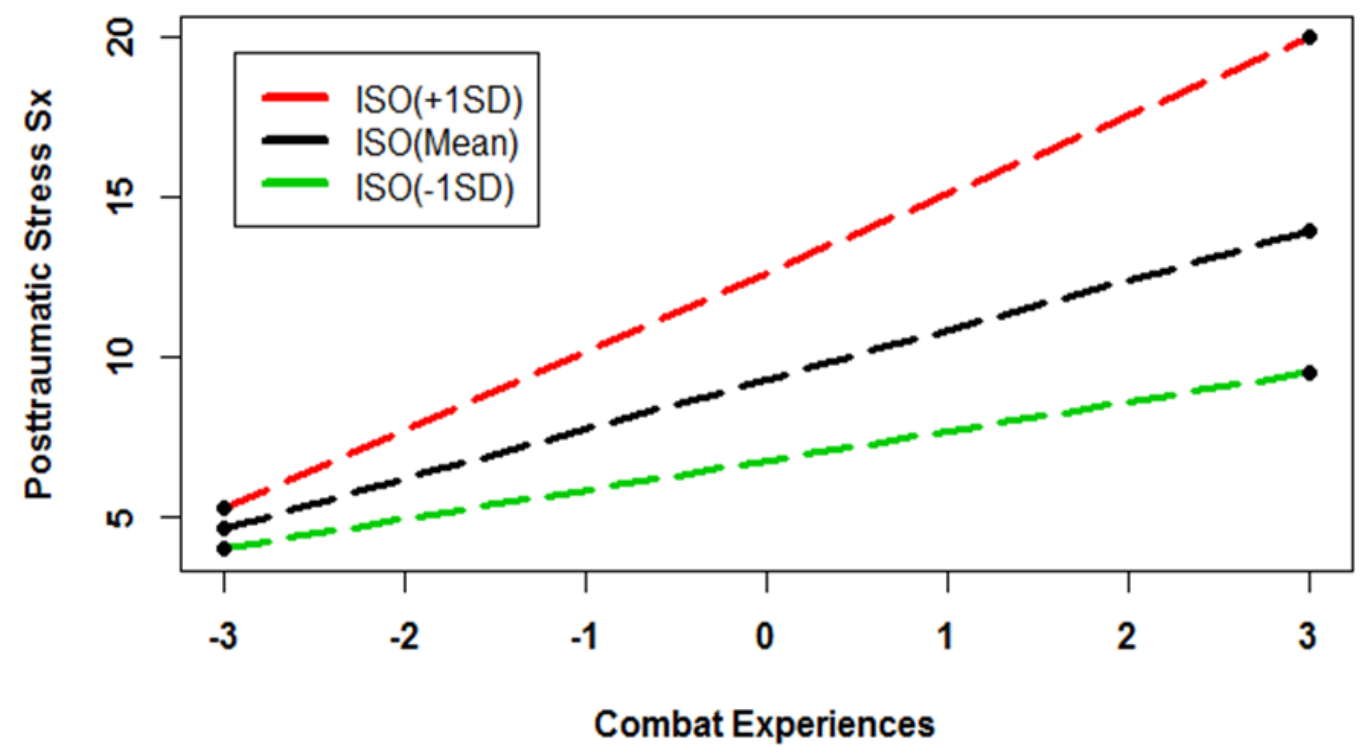

Figure 5. Hypothesis \#3: Sleep Quality will moderate Combat Experiences on PTSS

\section{Proposed Buffering effect of CESxSLEEP on PTSS}

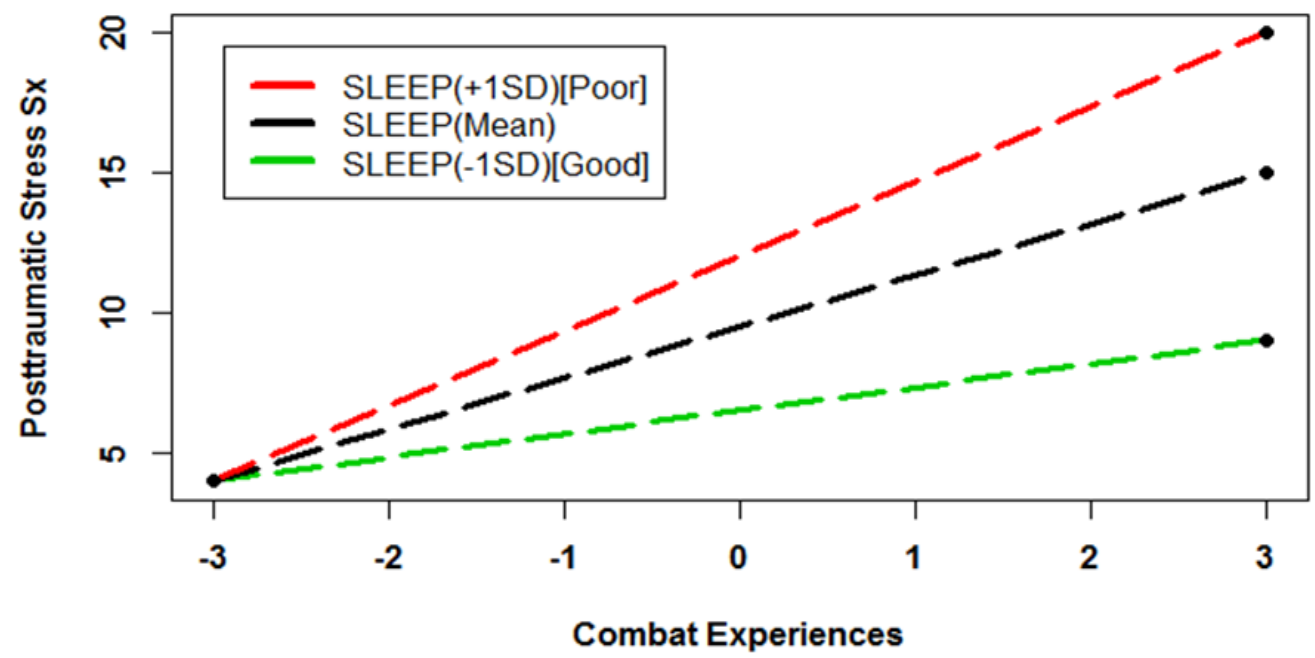


Table 2. Reflective EFA (27-items) Combat Experiences Scale (WRAIR, 2009) with "oblim" rotations

\begin{tabular}{|c|c|c|c|c|}
\hline Item & Description & $\begin{array}{c}1 \\
\text { (ATTA) }\end{array}$ & $\begin{array}{c}2 \\
(\mathrm{COMB})\end{array}$ & $\begin{array}{c}3 \\
\text { (LIND) }\end{array}$ \\
\hline ces01 & Being attacked or ambushed & $0.756^{*}$ & & \\
\hline ces02 & Receiving small arms fire & $0.666^{*}$ & & \\
\hline ces03 & Seeing dead bodies or human remains & 0301* & & \\
\hline ces04 & Seeing dead or seriously injured Americans & & & $0.407 *$ \\
\hline ces05 & Knowing someone seriously injured or killed & & & 0.658* \\
\hline $\operatorname{ces} 06$ & $\begin{array}{l}\text { Improvised explosive device (IED)/booby trap exploded } \\
\text { near you }\end{array}$ & & $0.360 *$ & \\
\hline ces07 & $\begin{array}{c}\text { Being physically moved or knocked over from an } \\
\text { explosion }\end{array}$ & & $0.524 *$ & \\
\hline $\operatorname{ces} 08$ & $\begin{array}{l}\text { Being in threatening situations where you were unable to } \\
\text { respond because of rules of engagement }\end{array}$ & & $0.355^{*}$ & \\
\hline ces09 & Shooting or directing fire at the enemy & & $0.747 *$ & \\
\hline $\operatorname{ces} 10$ & Engaging in hand-to-hand combat & & $0.406 *$ & \\
\hline ces11 & Clearing/searching homes or buildings & & $0.476 *$ & \\
\hline $\operatorname{ces} 12$ & $\begin{array}{l}\text { Witnessing brutality/mistreatment toward non- } \\
\text { combatants }\end{array}$ & & $0.555^{*}$ & \\
\hline ces13 & Being wounded/injured & & 0.559* & \\
\hline ces14 & $\begin{array}{c}\text { Seeing ill/injured women or children who you were } \\
\text { unable to help }\end{array}$ & & $0.464 *$ & \\
\hline ces15 & Receiving incoming artillery, rocket, or mortar fire & $0.694 *$ & & \\
\hline ces16 & $\begin{array}{c}\text { Being directly responsible for the death of an enemy } \\
\text { combatant }\end{array}$ & & $0.767 *$ & \\
\hline ces17 & $\begin{array}{l}\text { Feeling directly responsible for the death of a non- } \\
\text { combatant }\end{array}$ & & $0.727 *$ & \\
\hline ces18 & Feeling responsible for the death of US or ally personnel & & 0.428* & \\
\hline ces19 & Having a member of your own unit become a casualty & & & $0.810^{*}$ \\
\hline $\operatorname{ces} 20$ & $\begin{array}{c}\text { Had a close call, was shot or hit but protective gear } \\
\text { saved you }\end{array}$ & & $0.503 *$ & 0.463 \\
\hline ces21 & Had a buddy shot or hit who was near you & & 0.486* & \\
\hline ces22 & Had a close buddy seriously injured or killed & & & $0.772 *$ \\
\hline $\operatorname{ces} 23$ & Participating in IED/mine clearing operations & & & 0.309 \\
\hline ces24 & Saved the life of a Soldier or civilian & & & 0.452 \\
\hline $\operatorname{ces} 25$ & $\begin{array}{l}\text { Observing abuse of Laws of War/Geneva Convention } \\
\text { (e.g., weapons cached in Mosques, schools, or hospitals) }\end{array}$ & & & 0.554 \\
\hline ces26 & Encountering sniper fire & & & 0.648 \\
\hline ces27 & Believed you would be seriously injured or killed & $0.473 *$ & & \\
\hline
\end{tabular}




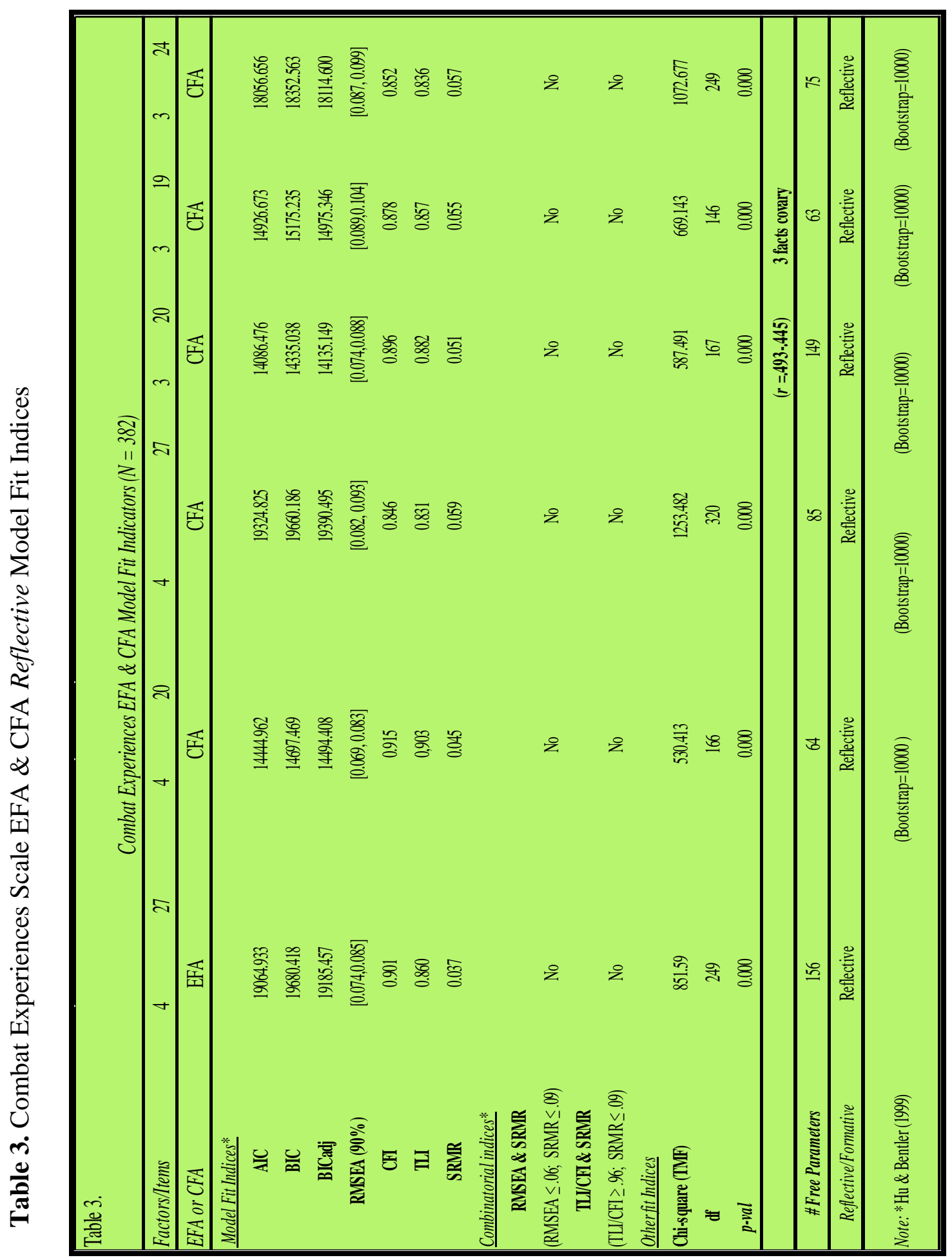


Figure 6. Four-factor formative Multiple Indicator Multiple Cause Combat Experiences Scale [(CFITLD>0.977; 90\%RMSEA $(0.037,0.136) ; \mathrm{AIC}=5446.407 ; \mathrm{BIC}=5534.219 ; \mathrm{BICadj}=5448.693 ;$ SRMR $=0.028 ;$ Dist=0.014 $]$

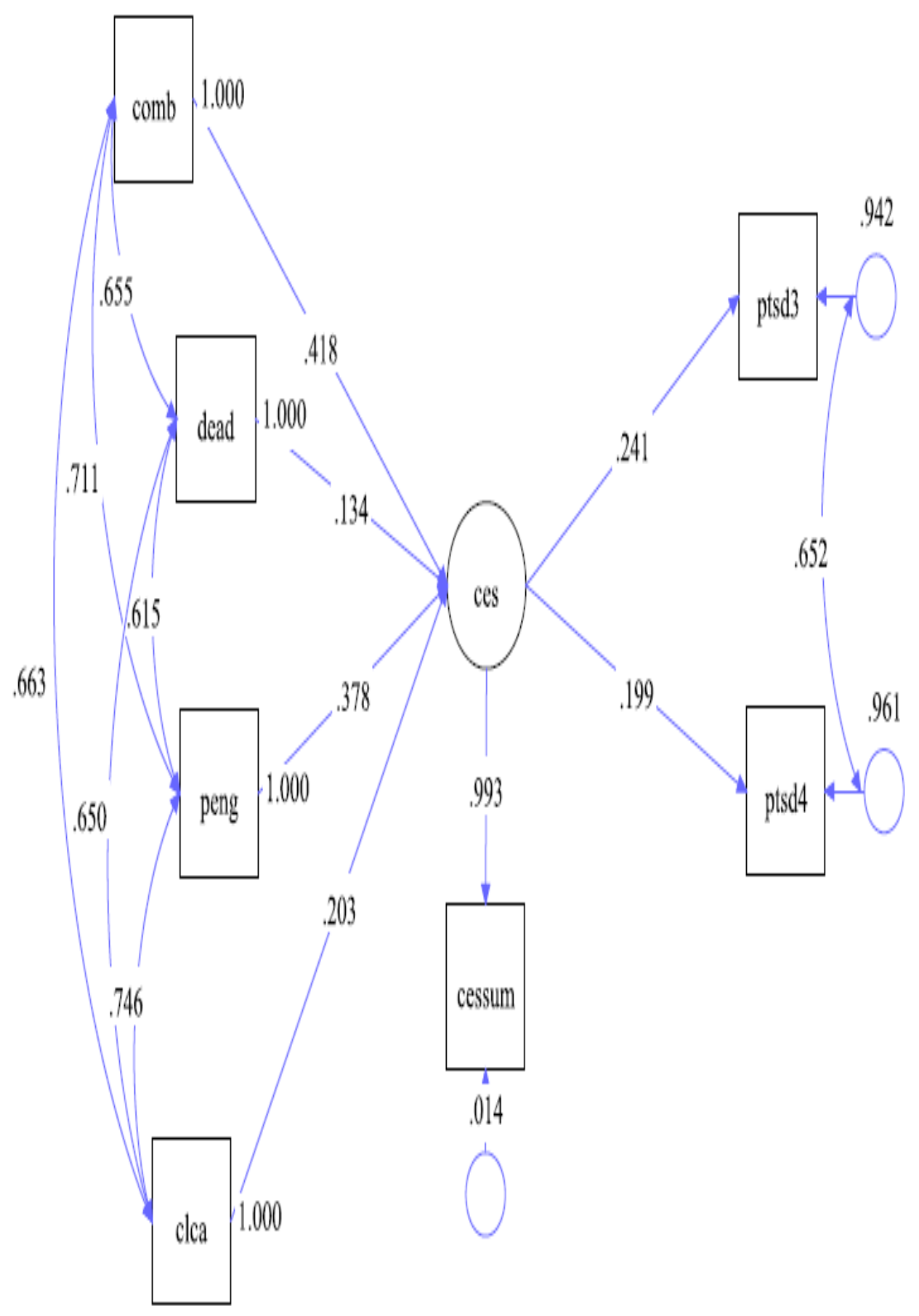




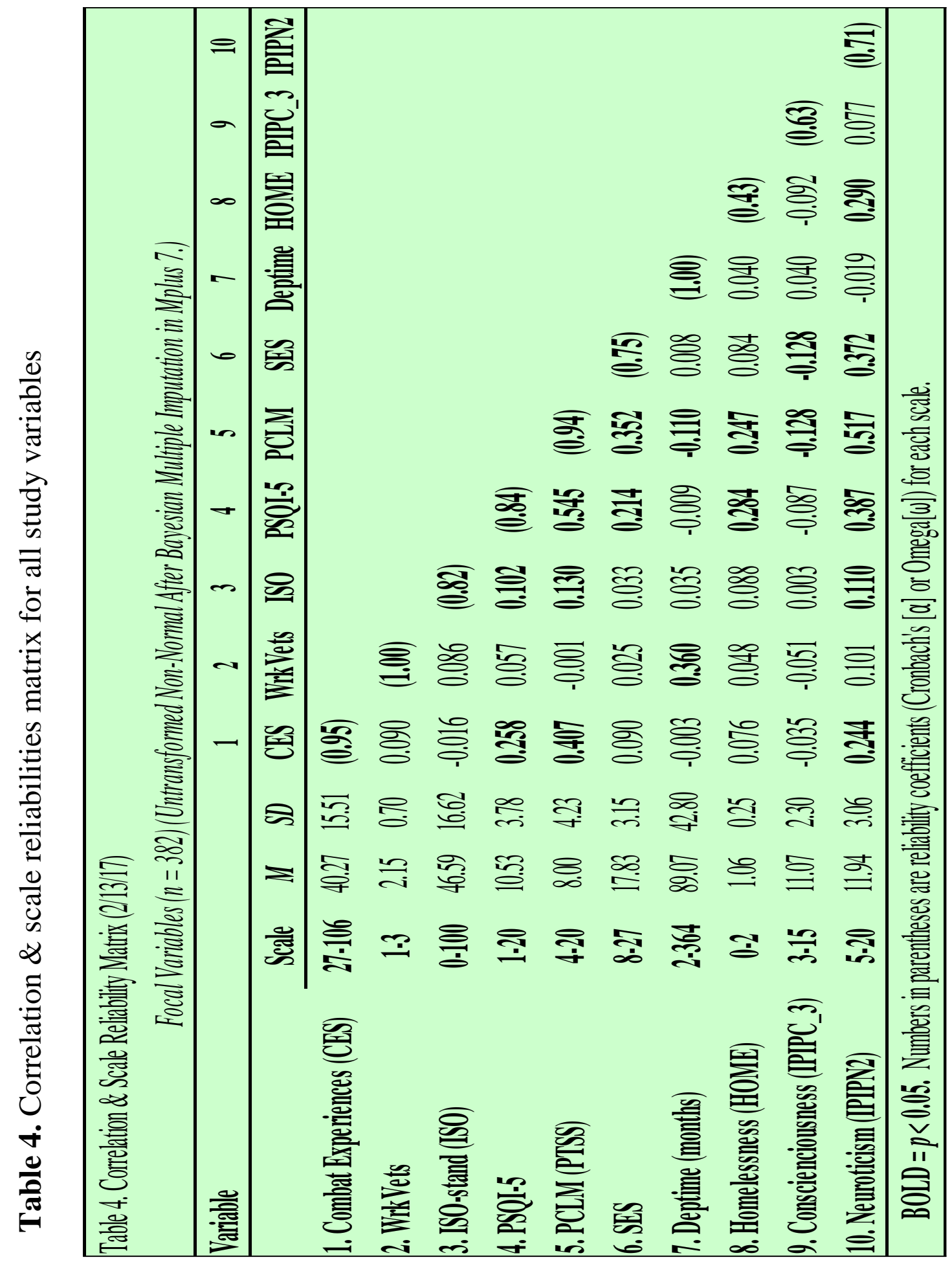




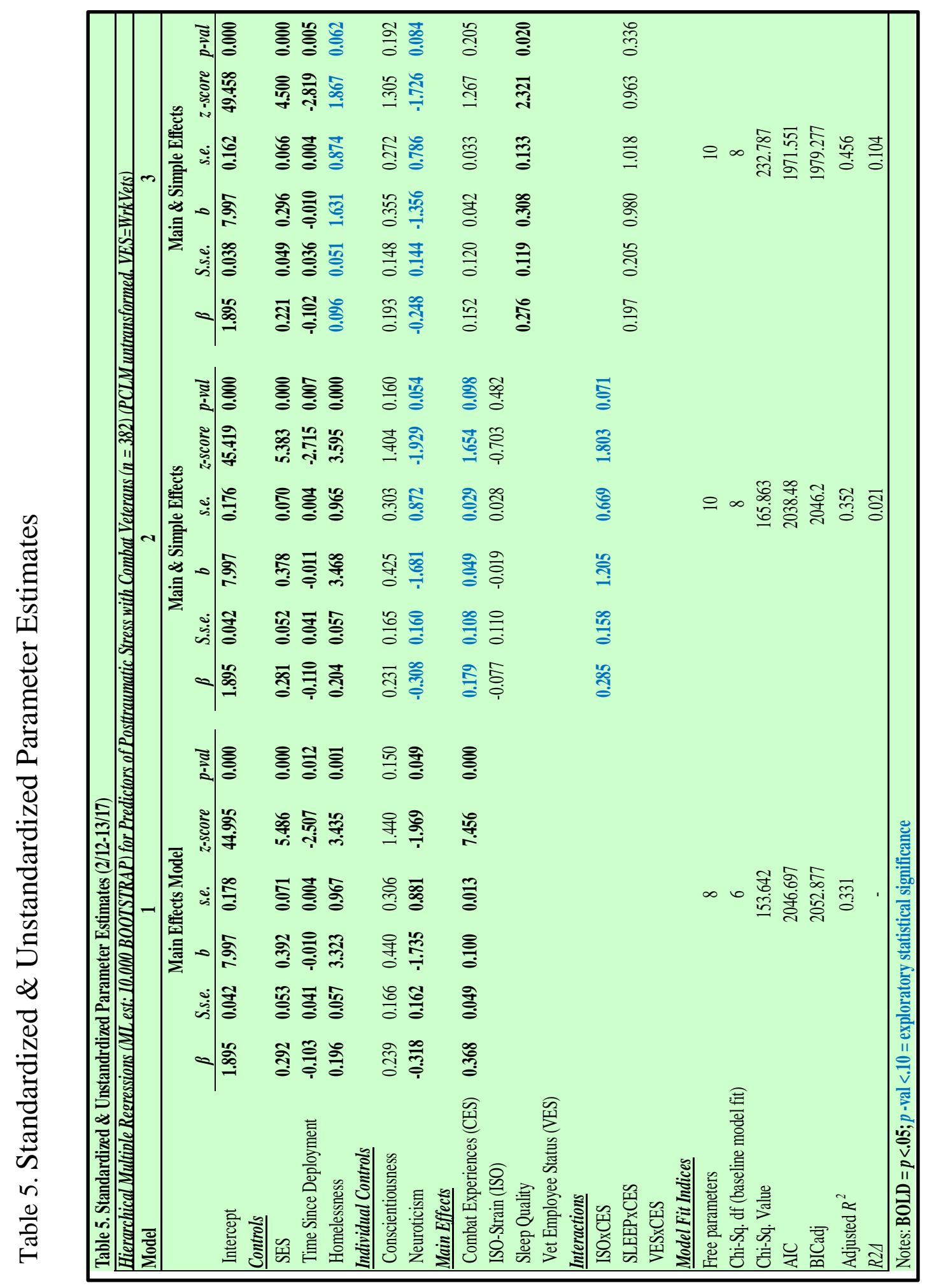




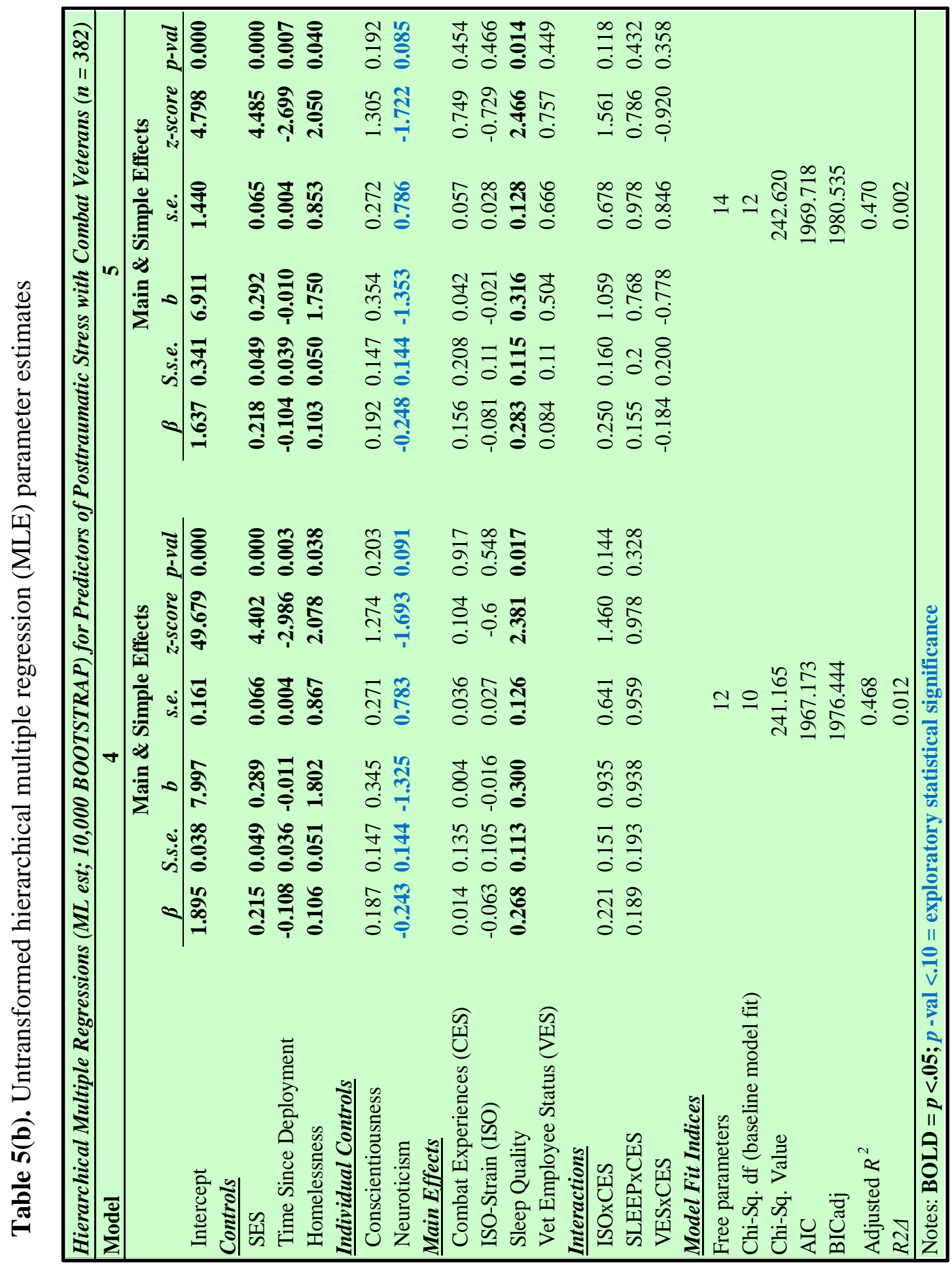




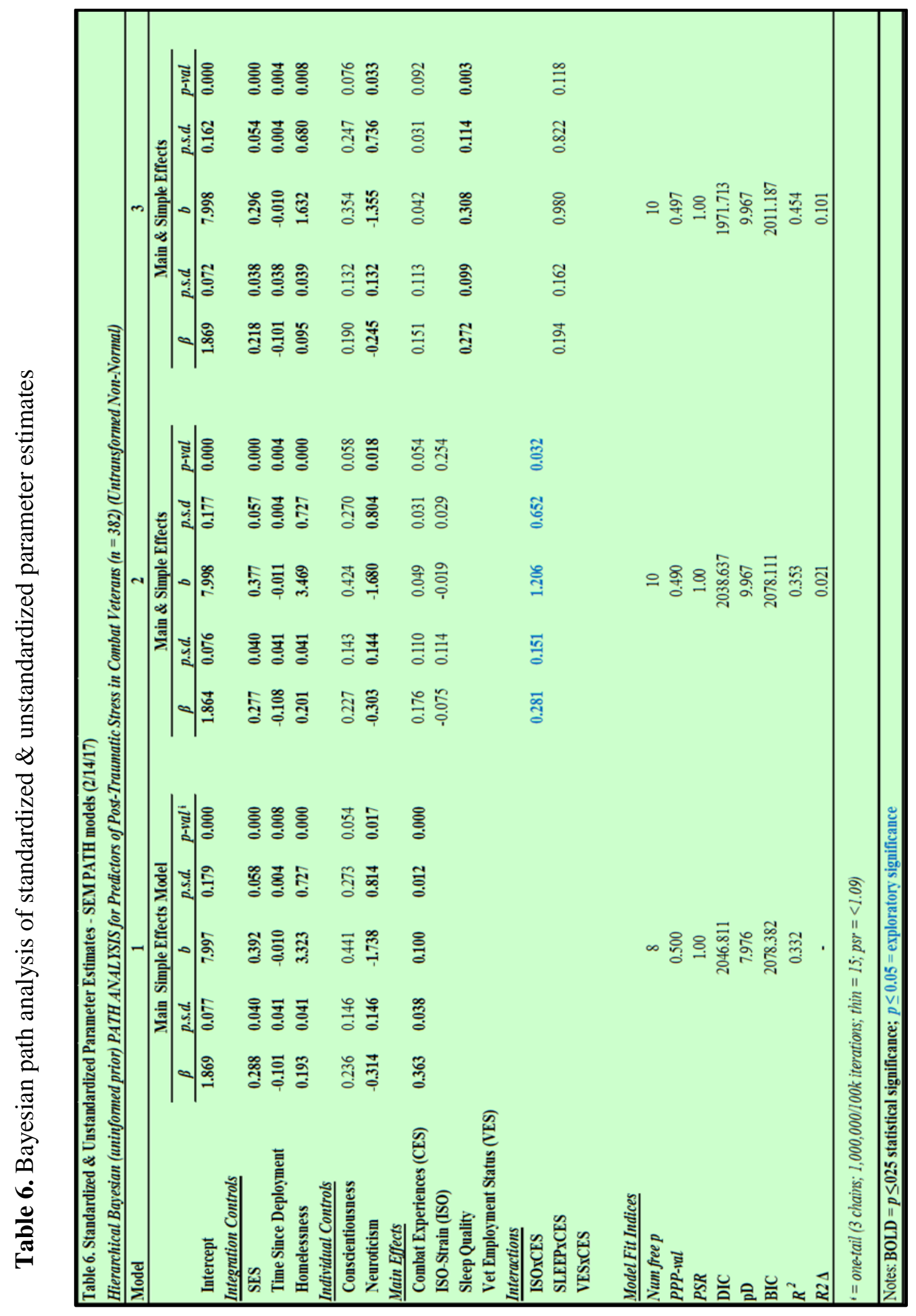




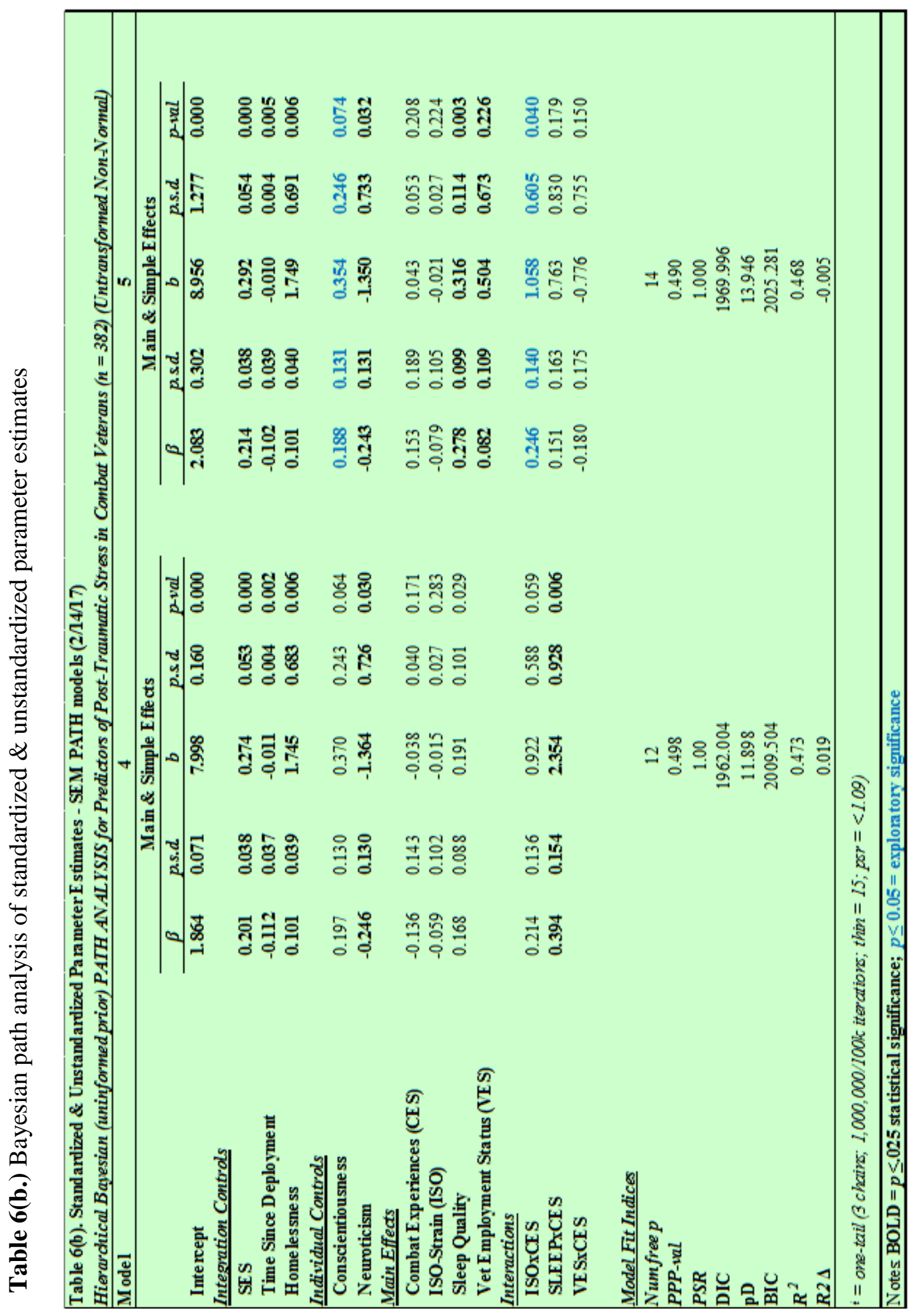


Figure 7. Three-group kernel density function plots of focal variables

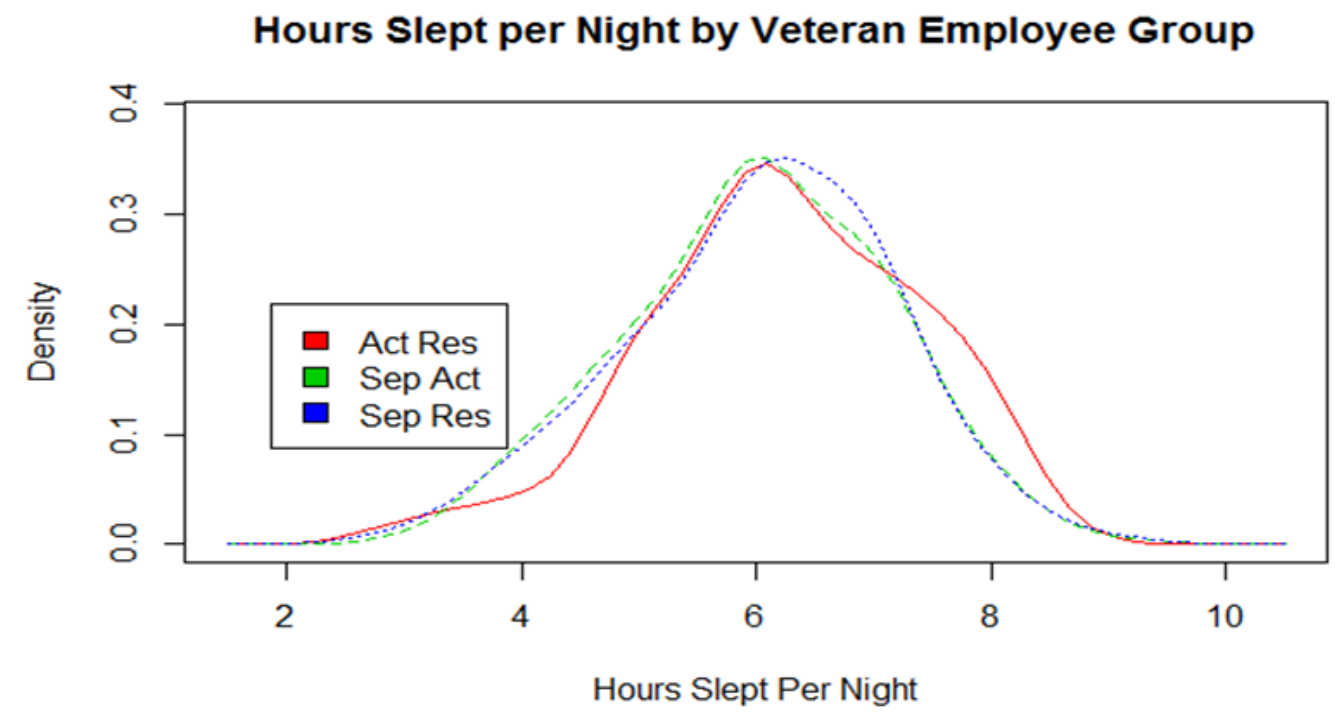

One-way ANOVA (equal variances not assumed)

$$
F_{2 / 177.45}=0.900, p=n . s \text {. }
$$

Figure 8. Three-group kernel density function plots of focal variables

Iso-strain by Veteran Employee Group

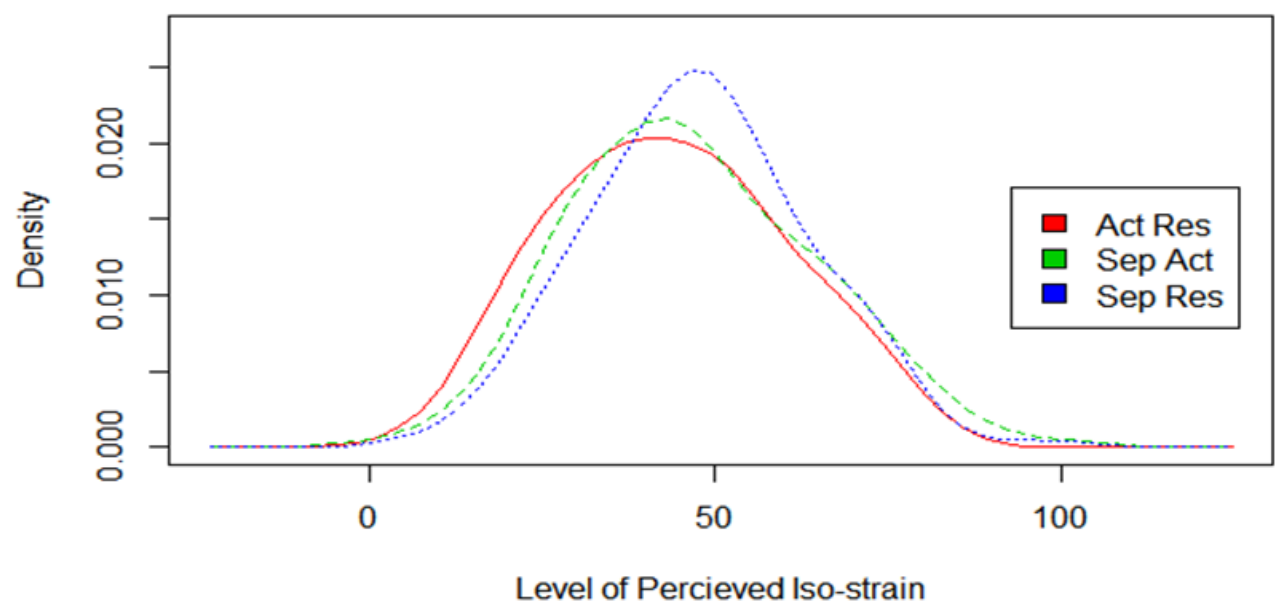

One-way ANOVA (equal variances not assumed)

$$
F_{2 / 182.09}=1.430, p=n . s .
$$


Figure 9. Three-group kernel density function plots of focal variables

PTSS by Veteran Employee Group

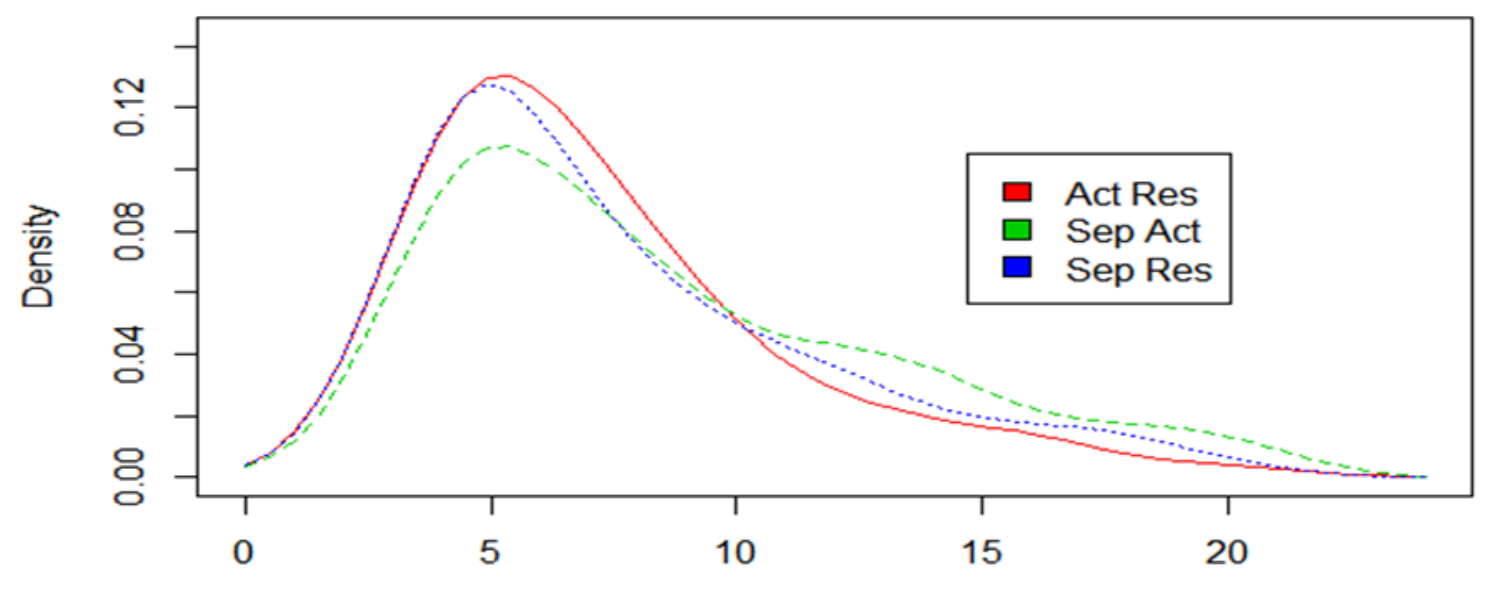

Level of Postraumatic Stress Symptoms

One-way ANOVA (equal variances not assumed)

$F_{2 / 193.92}=3.388, p<.05^{*}$

Figure 10. Three-group kernel density function plots of focal variables

Combat Experiences by Veteran Employee Group

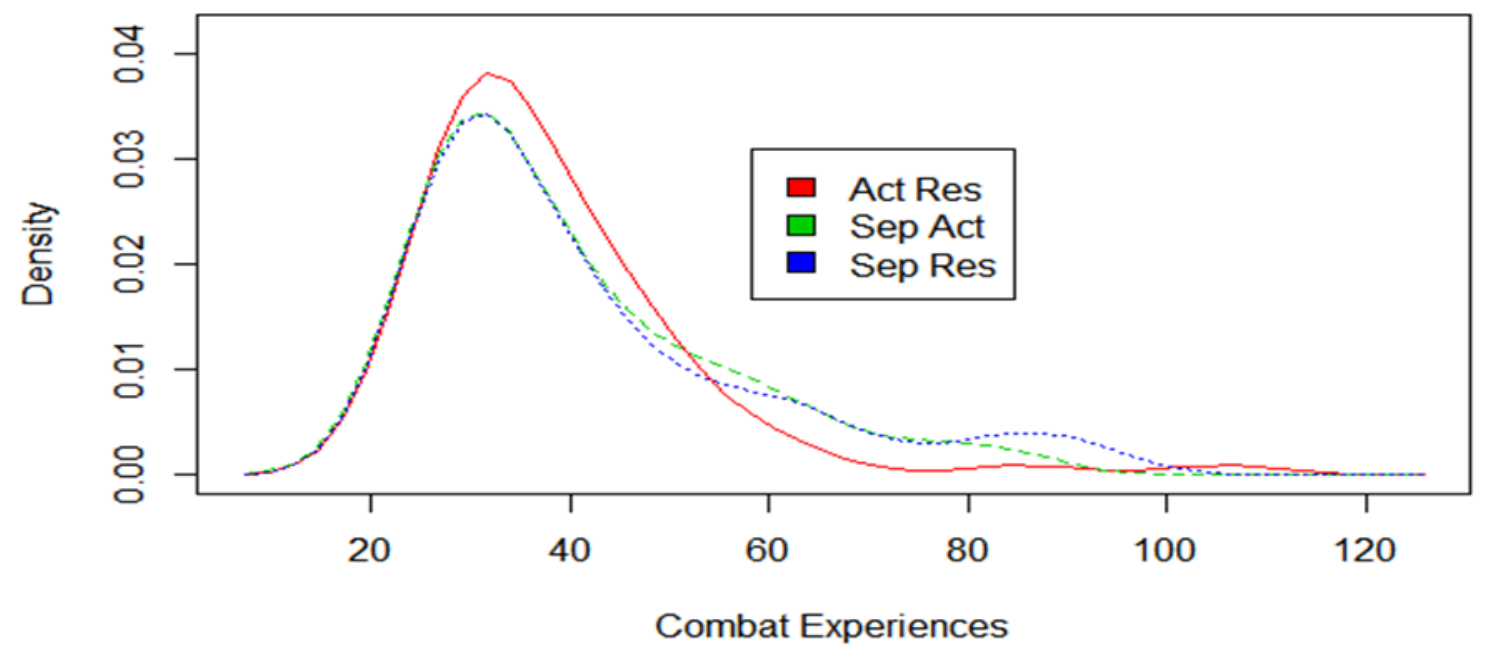

One-way ANOVA (equal variances not assumed)

$$
F_{2 / 185.33}=1.254, p=n . s .
$$


Figure 11. Three-group kernel density function plots of focal variables

\section{Sleep Quality by Veteran Employee Group}

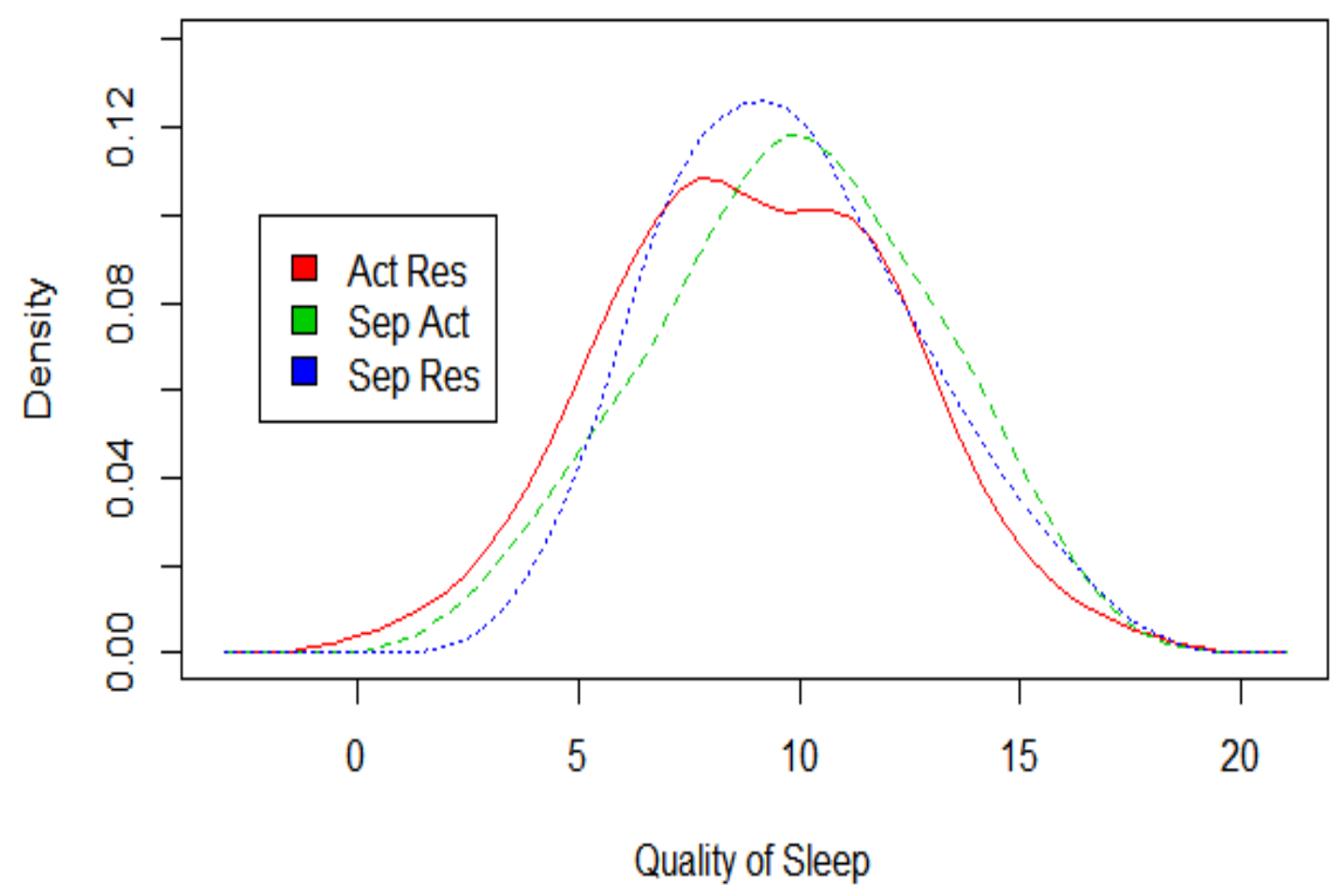

One-way ANOVA (equal variances not assumed)

$$
F_{2 / 177.86}=1.830, p=\text { n.s. }
$$


Figure 12. Prevalence of "probable PTSD" among SERVe combat veterans at baseline

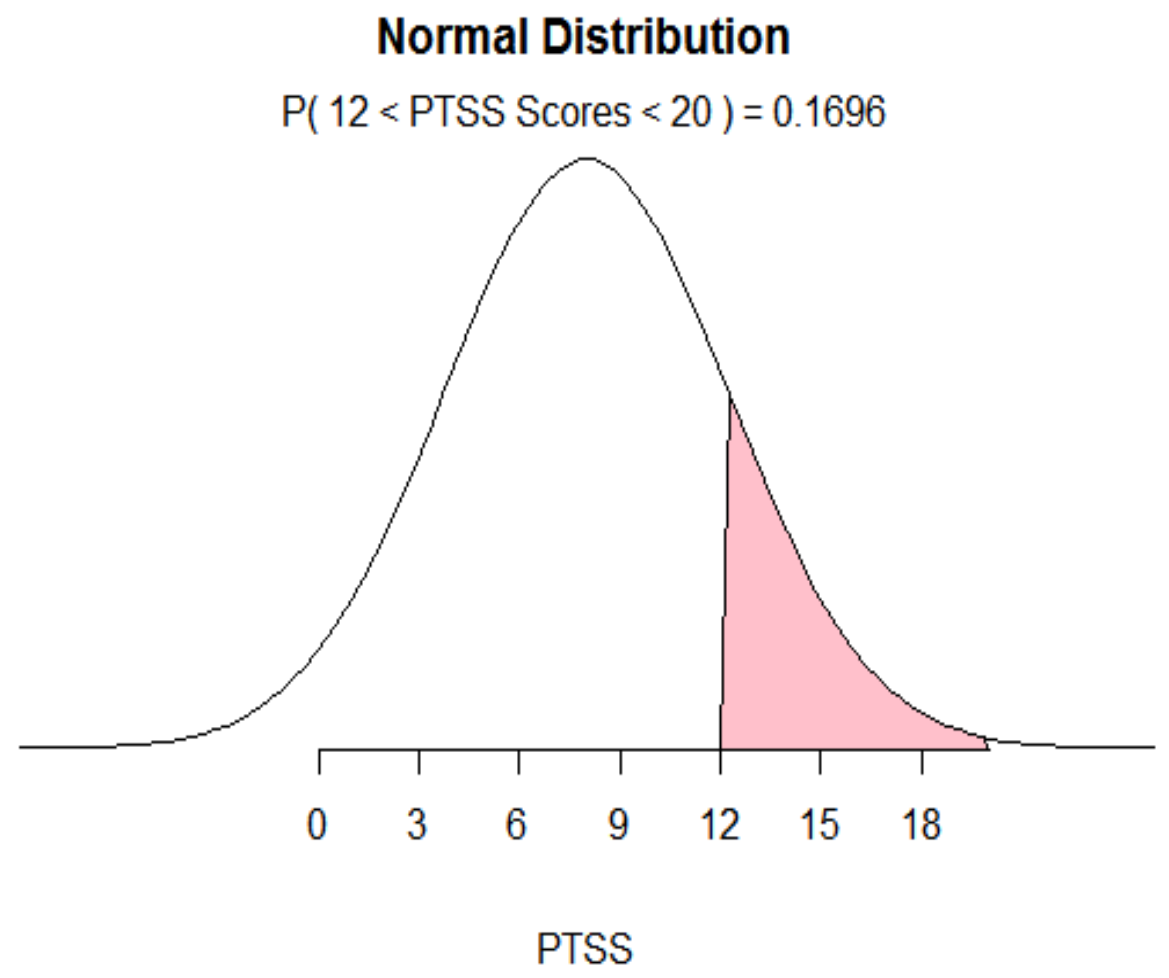

*Note - based on cut score of "12" (Bliese et al., 2011) 
Figure 13. Combat Experiences $x$ Iso-strain on symptoms of posttraumatic stress

\section{2-Way Enhancing Effect of CESxISO}

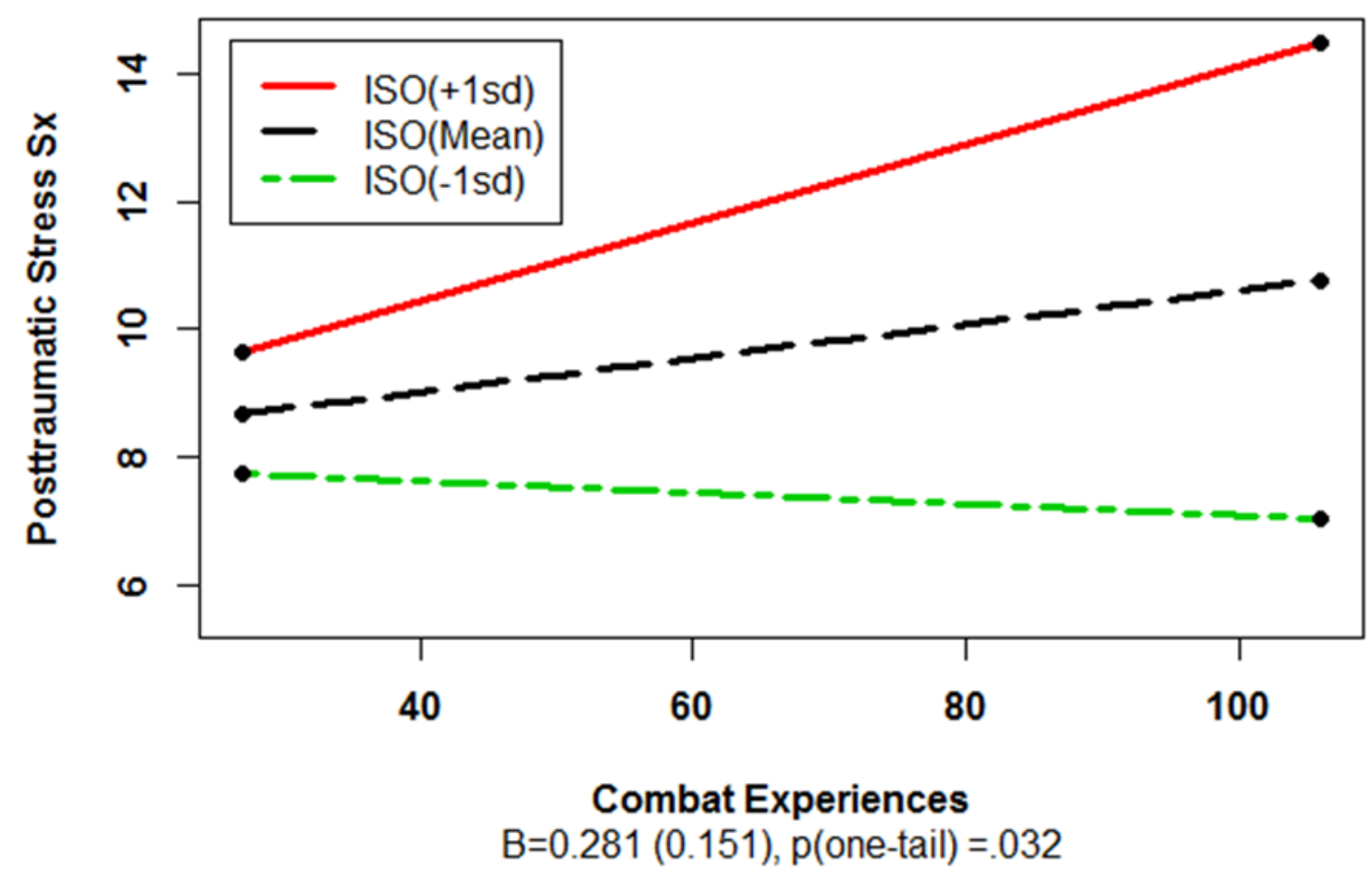

*Note: Bayesian Path Analyses Table 6./Model 2.

Fit indices $[n f p=10 ; P P P$-val $=0.490 ; P S R=1.00 ; D I C=2038.637 ; p D=9.967$;

$$
\left.B I C=2078.111 ; R^{2}=0.353\right]
$$


Figure 14. Combat Experiences $x$ Sleep Quality on symptoms of posttraumatic stress

\section{2-Way Buffering Effect of CESxSLEEP}

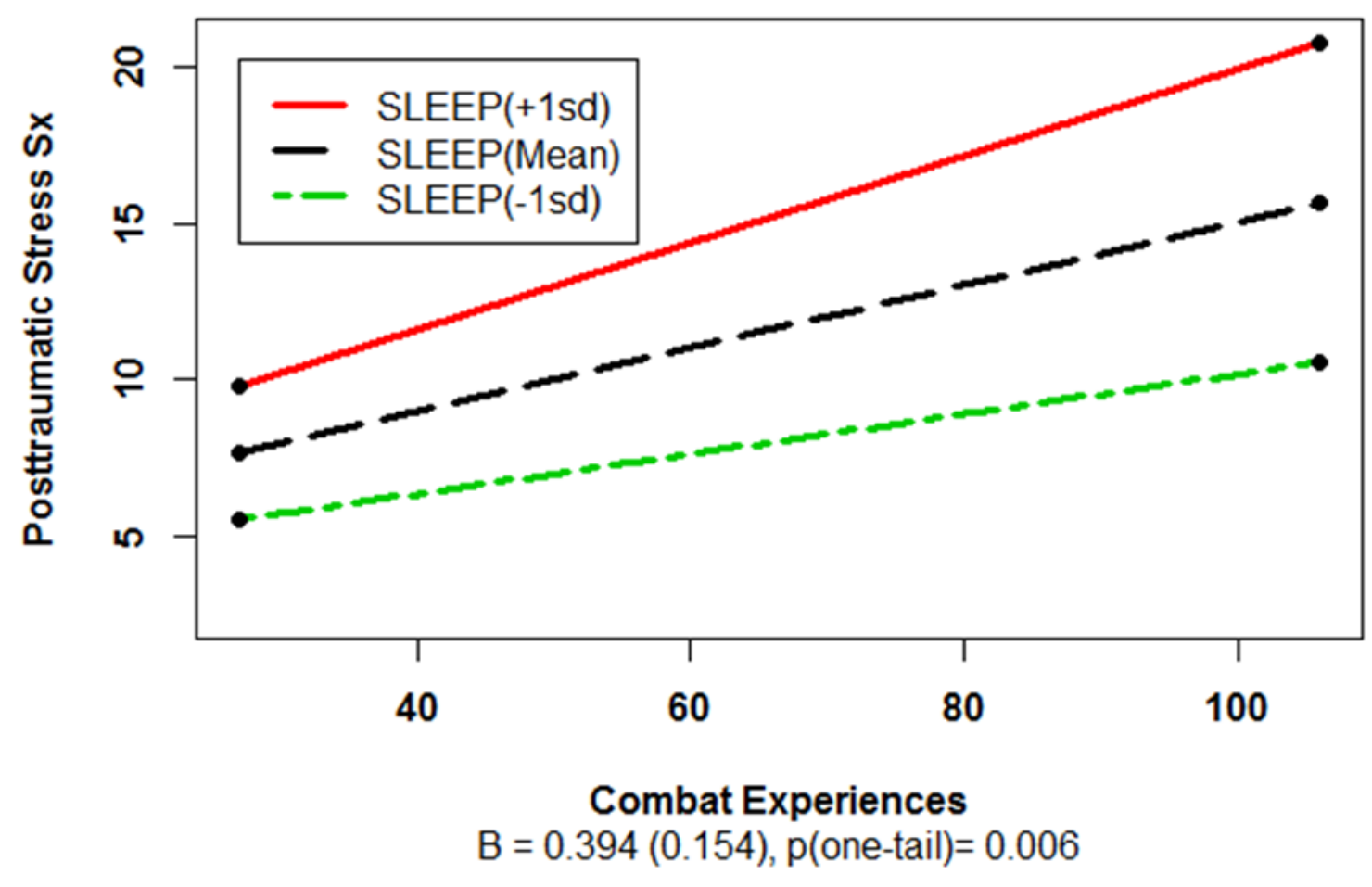

*Note: Bayesian Path Analyses Table 6b./Model 4.

Fit indices $[n f p=12 ; P P P$-val $=0.498 ; P S R=1.00 ; D I C=1962.004 ; p D=11.898$;

$$
\left.B I C=2009.504 ; R^{2}=0.473\right]
$$


Figure 15. Bayesian path model of Combat Experiences, Iso-strain, \& Sleep Quality on PTSS

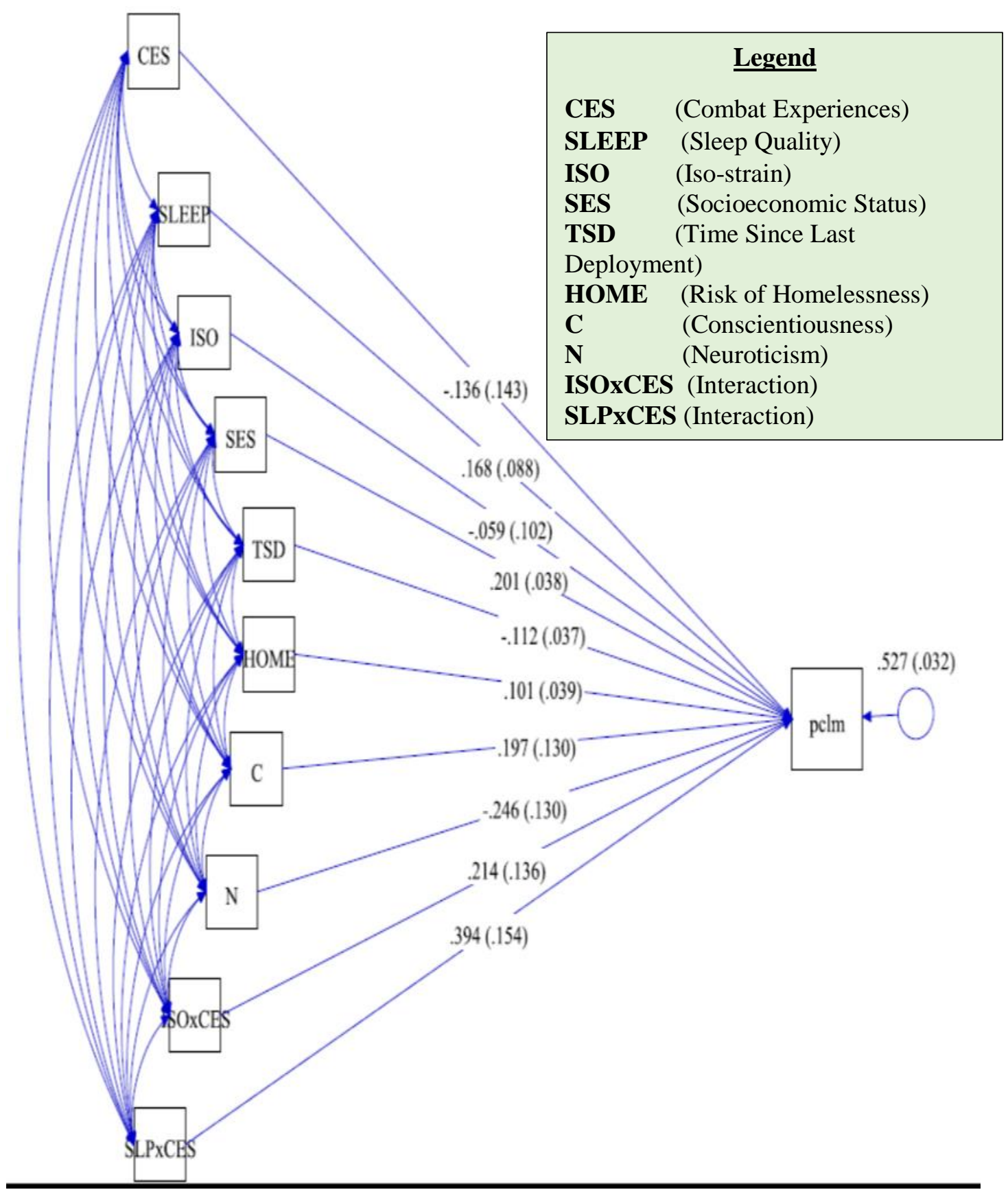

*Note: Bayesian Path Analyses Table 6b./Model 4.

Fit indices $[n f p=12 ; P P P-$ val $=0.498 ; P S R=1.00 ; D I C=1962.004 ; p D=11.898 ; B I C=2009.504 ; R$ $\left.{ }^{2}=\mathbf{0 . 4 7 3}\right]$ 
Figure 16. Single-group $F$-test power analysis using five tested predictors

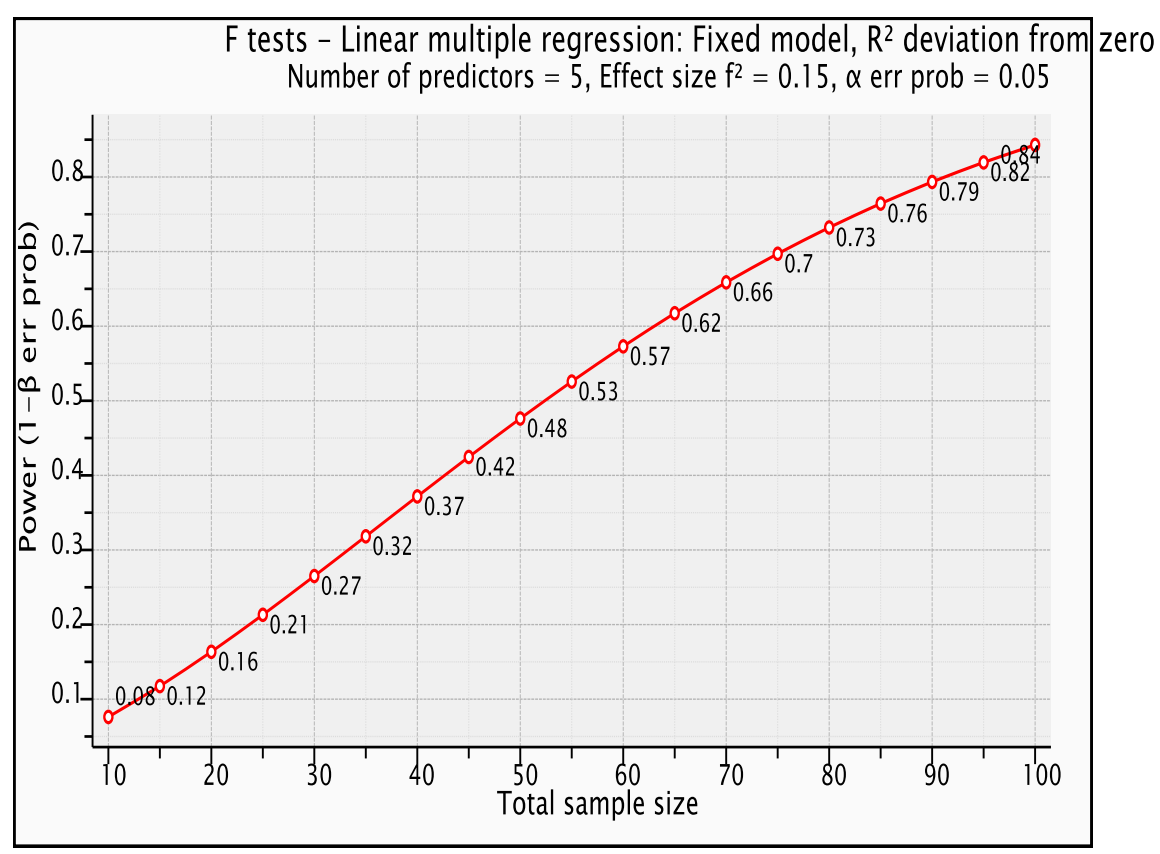




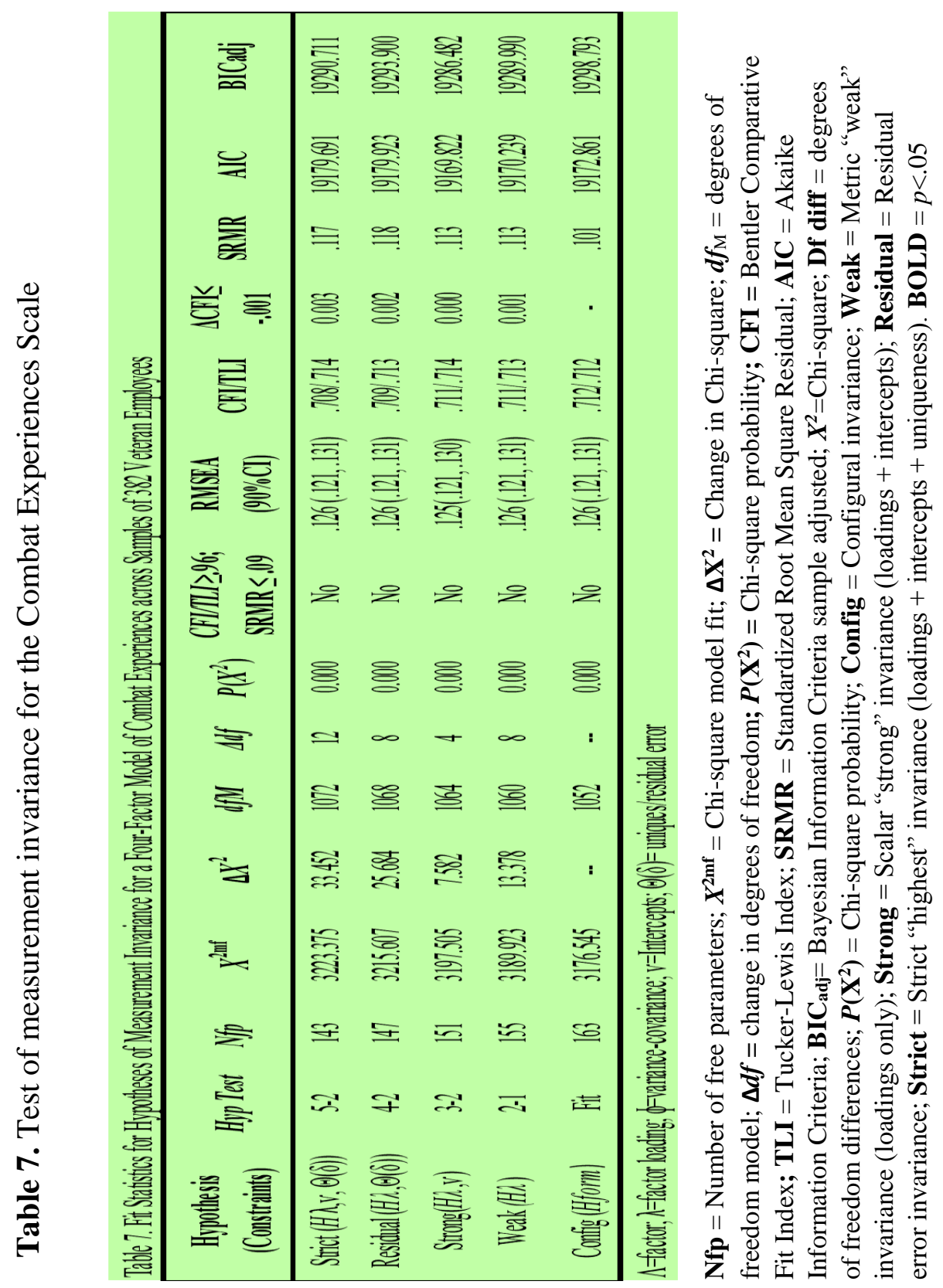




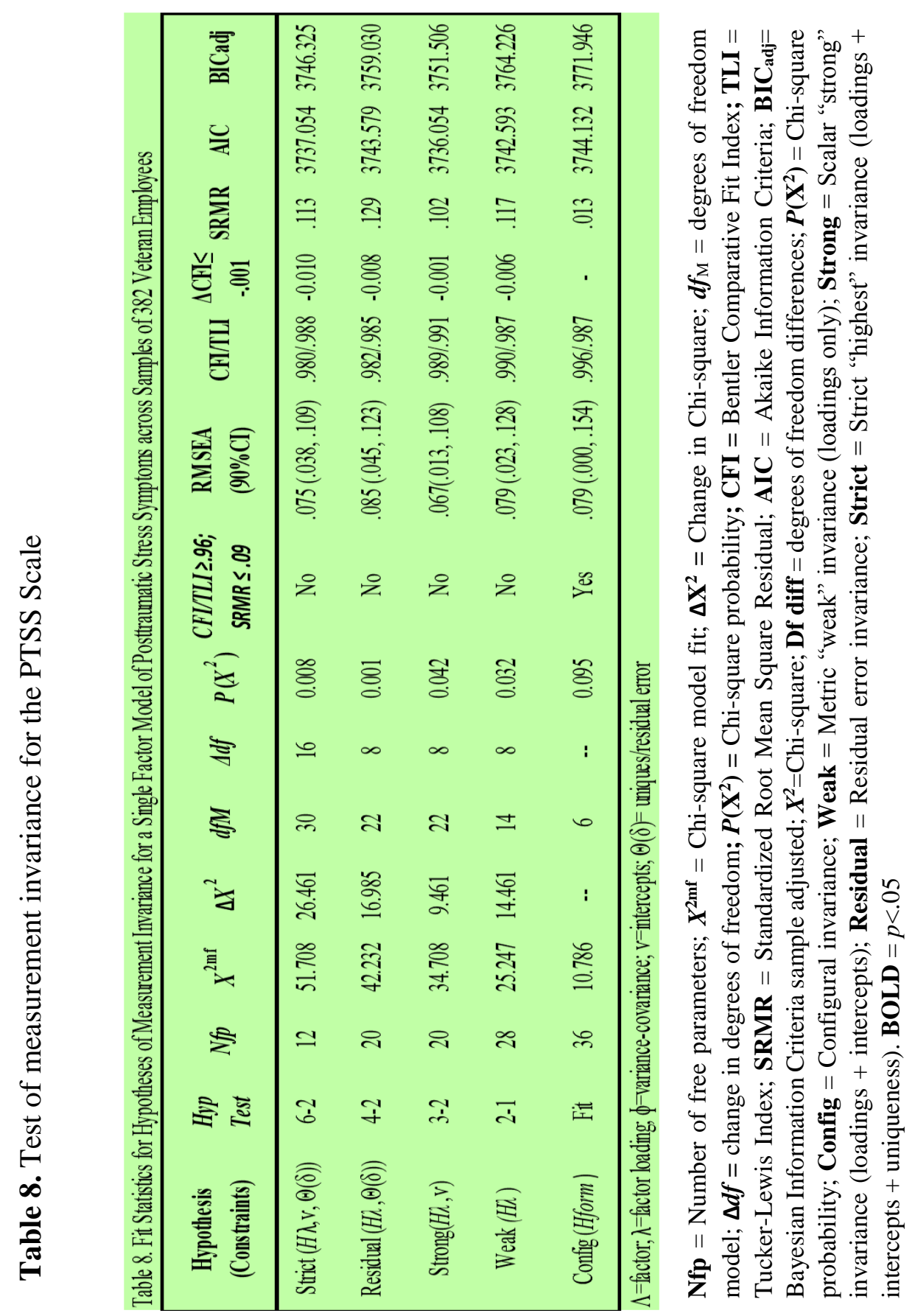


Figure 17. Demographics: Post-9/11 veterans by age group

\section{Post-9/11 Veterans by age group}

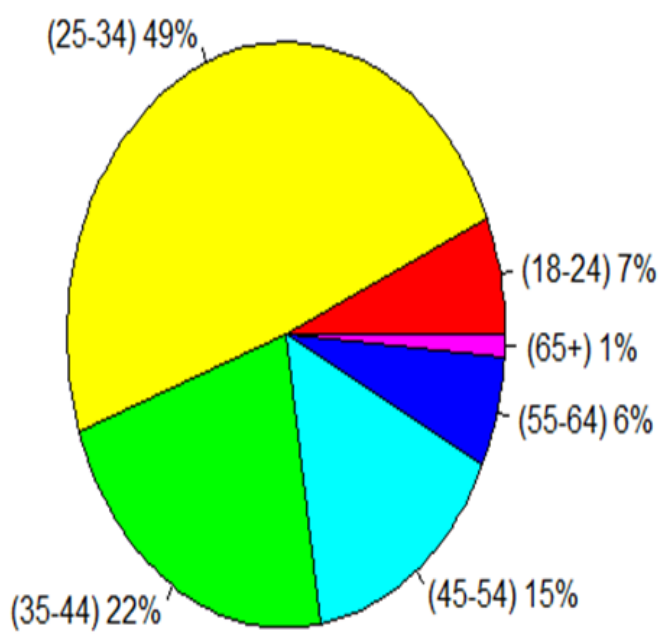

Source: U.S. Bureau of Labor (2015) 
Figure 18. Iso-strain (Mathers et al.) by Iso-strain (Brady adapted from Mathers)

\section{ISO (SCHCONT) by ISO (W/O SCHCONT}

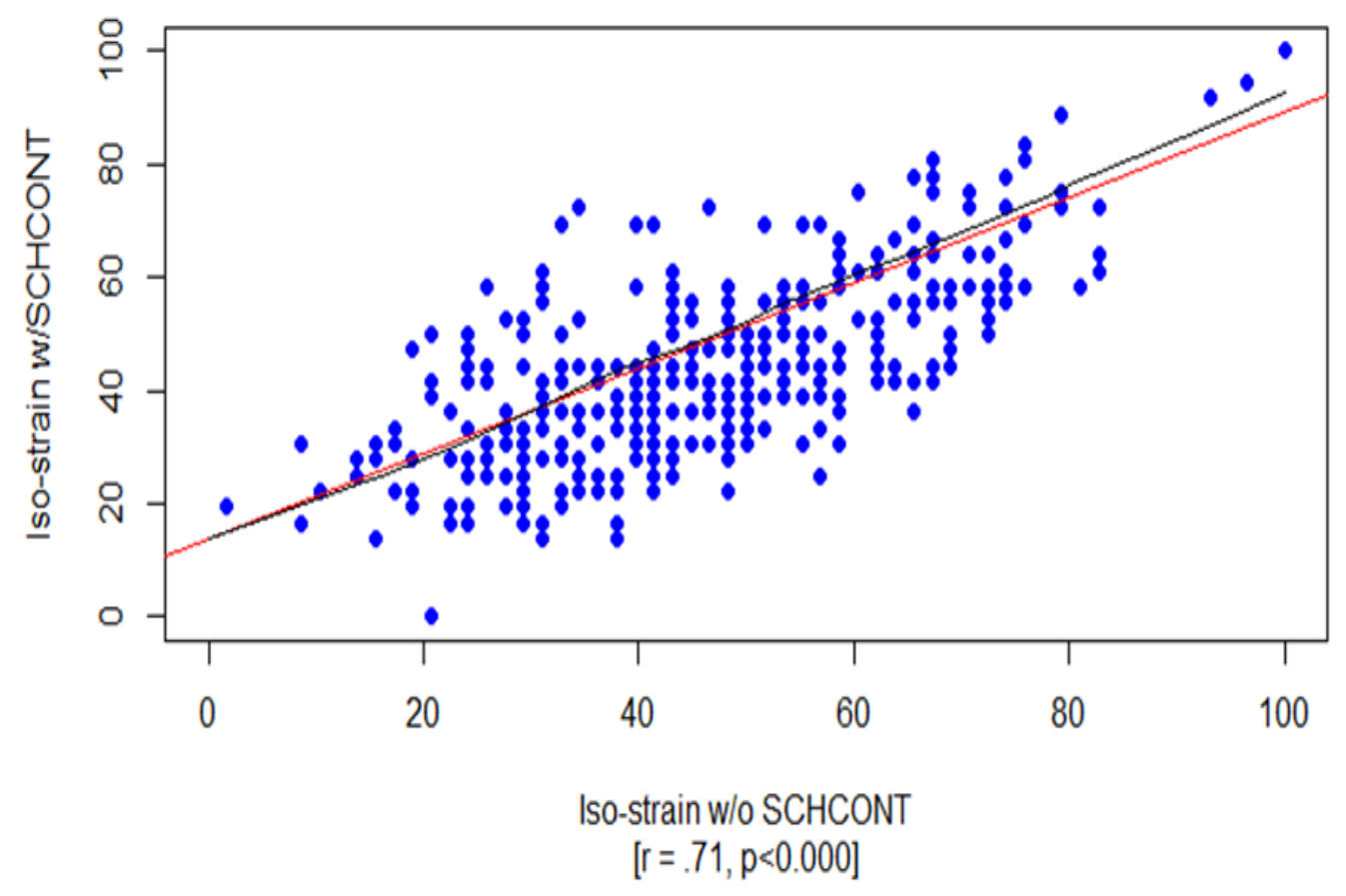


Combat Experiences, Iso-strain, and Sleep Quality Affect PTSS

\section{References}

Adler, A. B., Bliese, P. D., Pickering, M. A., Hammermeister, J., Williams, J., Harada, C., ... Ohlson, C. (2015). Mental skills training with basic combat training soldiers: A group-randomized trial. The Journal of Applied Psychology, 100(6), 1752-1764. https://doi.org/10.1037/ap10000021

Adler, A. B., \& Castro, C. A. (2013). An Occupational Mental Health Model for the Military. Military Behavioral Health, 1(1), 41-45. https://doi.org/10.1080/21635781.2012.721063

Adler, D. A., Possemato, K., Mavandadi, S., Lerner, D., Chang, H., Klaus, J., ... Oslin, D. W. (2011). Psychiatric Status and Work Performance of Veterans of Operations Enduring Freedom and Iraqi Freedom. Psychiatric Services, 62(1), 39-46. https://doi.org/10.1176/appi.ps.62.1.39

Afifi, T. O., Taillieu, T., Zamorski, M. A., Turner, S., Cheung, K., \& Sareen, J. (2016). Association of Child Abuse Exposure With Suicidal Ideation, Suicide Plans, and Suicide Attempts in Military Personnel and the General Population in Canada. JAMA Psychiatry, 73(3), 229-238.

https://doi.org/10.1001/jamapsychiatry.2015.2732

Akerstedt, T. (2003). Shift work and disturbed sleep/wakefulness. Occupational Medicine, 53(2), 89-94. https://doi.org/10.1093/occmed/kqg046

Akerstedt, T., Fredlund, P., Gillberg, M., \& Jansson, B. (2002). A prospective study of fatal occupational accidents -- relationship to sleeping difficulties and occupational factors. Journal of Sleep Research, 11(1), 69-71. 
Akerstedt, T., Fredlund, P., Gillberg, M., \& Jansson, B. (2002). Work load and work hours in relation to disturbed sleep and fatigue in a large representative sample. Journal of Psychosomatic Research, 53(1), 585-588.

Akerstedt, T., Knutsson, A., Westerholm, P., Theorell, T., Alfredsson, L., \& Kecklund, G. (2002). Sleep disturbances, work stress and work hours: a cross-sectional study. Journal of Psychosomatic Research, 53(3), 741-748.

Åkerstedt, T., Knutsson, A., Westerholm, P., Theorell, T., Alfredsson, L., \& Kecklund, G. (2004). Mental fatigue, work and sleep. Journal of Psychosomatic Research, 57(5), 427-433. https://doi.org/10.1016/j.jpsychores.2003.12.001

Allen, K. (2003). Are pets a healthy pleasure? the influence of pets on blood pressure. Current Directions in Psychological Science, 12(6), 236-239. https://doi.org/10.1046/j.0963-7214.2003.01269.x

Alves, M. G. de M., Braga, V. M., Faerstein, E., Lopes, C. S., \& Junger, W. (2015). The demand-control model for job strain: a commentary on different ways to operationalize the exposure variable. Cadernos de Saúde Pública, 31(1), 208-212. https://doi.org/10.1590/0102-311X00080714

American Psychiatric Association. (2013). Management of adults with traumatic brain injury. (D. B. Arciniegas, Ed.) (1st ed). Washington, DC: American Psychiatric Pub.

American Psychiatric Association, \& American Psychiatric Association. (1980). Diagnostic and statistical manual of mental disorders: DSM-III. Washington, DC: American Psychiatric Association. 
Combat Experiences, Iso-strain, and Sleep Quality Affect PTSS

American Psychiatric Association, \& American Psychiatric Association. (2013).

Diagnostic and statistical manual of mental disorders: DSM-5 (5th ed).

Washington, D.C: American Psychiatric Association.

American Psychiatric Association, American Psychiatric Association, \& Task Force on

DSM-IV. (1994). Diagnostic and statistical manual of mental disorders: DSM-IV.

Washington, DC: American Psychiatric Association.

Anderson, K., \& Mitchell, J. (1992). Effects of military experience on mental health problems and work behavior. Medical Care, (30), 554:563.

Anderson, P. (2014). Unemployment biggest predictor of PTSD symptom severity

(Psychiatry). Medscape. Retrieved from

www.medscape.com/viewarticle/824747_print

Anderson, S. E., Coffey, B. S., \& Byerly, R. T. (2002). Formal Organizational Initiatives and Informal Workplace Practices: Links to Work-Family Conflict and Job-Related Outcomes. Journal of Management, 28(6), 787-810.

https://doi.org/10.1177/014920630202800605

Ashkenas, J., \& Parlapiano, A. (2014, June 6). How the Recession Reshaped the Economy, in 255 Charts. The New York Times. New York, N.Y.

Atkins, D. M. (2013, July 11). Florida National Guard marks 100th deployment since 9/11. The Miami Herald. Retrieved from www.miamiherald.com/2013/07/11/vprint/3495849/florida-national-guard-marks-100th.html 
Combat Experiences, Iso-strain, and Sleep Quality Affect PTSS

Atkinson, A. C. (1994). Plots, transformations, and regression: an introduction to graphical methods of diagnostic regression analysis (Reprinted). Oxford: Oxford Univ. Press.

Bakker, A. B., \& Demerouti, E. (2007). The Job Demands-Resources model: state of the art. Journal of Managerial Psychology, 22(3), 309-328. https://doi.org/10.1108/02683940710733115

Bakker, A. B., Hakanen, J. J., Demerouti, E., \& Xanthopoulou, D. (2007). Job resources boost work engagement, particularly when job demands are high. Journal of Educational Psychology, 99(2), 274-284. https://doi.org/10.1037/00220663.99.2.274

Barlow, D., \& Durand, M. (2002). Essentials of Abnormal Psychology (3rd ed.).

Barnes, C. M. (2012). Working in our sleep: Sleep and self-regulation in organizations. Organizational Psychology Review, 2(3), 234-257.

https://doi.org/10.1177/2041386612450181

Baron, R. M., \& Kenny, D. A. (1986). The moderator-mediator variable distinction in social psychological research: Conceptual, strategic, and statistical considerations. Journal of Personality and Social Psychology, 51(6), 1173-1182. https://doi.org/10.1037/0022-3514.51.6.1173

Bartone, P. (1995). A Short Hardiness Scale. In meeting of (p. 5). New York, NY: Walter Reeed Army Institute of Research US Army Medical Research Unit : Germany. Retrieved from http://www.hardinessresilience.com/docs/APS95HAN1.pdf 
Combat Experiences, Iso-strain, and Sleep Quality Affect PTSS

Bartone, P. (1999). Hardiness protects against war-related stress in Army Reserve Forces. Consulting Psychology Journal, 51(2), 72-82.

Bartone, P. T., Kelly, D. R., \& Matthews, M. D. (2013). Psychological Hardiness Predicts Adaptability in Military Leaders: A prospective study: Hardiness and Leader Adaptability. International Journal of Selection and Assessment, 21(2), 200-210. https://doi.org/10.1111/ijsa.12029

Bartone, P. T., Marlowe, D., Gifford, R., \& Wright, K. (1992). Personality hardiness predicts soldier adjustment to combat stress. In Personality hardiness predicts soldier adjustment to combat stress. Willamsburg, VA.

Basta, M., Chrousos, G. P., Vela-Bueno, A., \& Vgontzas, A. N. (2007). Chronic Insomnia and the Stress System. Sleep Medicine Clinics, 2(2), 279-291. https://doi.org/10.1016/j.jsmc.2007.04.002

Beard, G. M. (1881). American Nervousness Its Causes and Consequences: A supplement to Nervous Exhaustion (Neurasthenia). 27 \& 29 West 23D Street, NY: G.P. Putnam's Sons.

Beck, S. L., Schwartz, A. L., Towsley, G., Dudley, W., \& Barsevick, A. (2004). Psychometric evaluation of the Pittsburgh sleep quality index in cancer patients. Journal of Pain and Symptom Management, 27(2), 140-148. https://doi.org/10.1016/j.jpainsymman.2003.12.002

Behson, S. J. (2005). The relative contribution of formal and informal organizational work-family support. Journal of Vocational Behavior, 66(3), 487-500. https://doi.org/10.1016/j.jvb.2004.02.004 
Bergman, M. E., \& Jean, V. A. (2016). Where Have All the "Workers" Gone? A Critical Analysis of the Unrepresentativeness of Our Samples Relative to the Labor Market in the Industrial-Organizational Psychology Literature. Industrial and Organizational Psychology, 9(01), 84-113. https://doi.org/10.1017/iop.2015.70

Berkman, L., \& Syme, L. (1979). Social networks, host resistance, and mortality: A nine-year follow-up study of Almeda County residents. American Journal of Epidemiology, 109(2), 186.

Berry, J. W. (1997). Immigration, Acculturation, and Adaptation. Applied Psychology, 46(1), 5-34. https://doi.org/10.1111/j.1464-0597.1997.tb01087.x

Berset, M., Elfering, A., Lüthy, S., Lüthi, S., \& Semmer, N. K. (2011). Work stressors and impaired sleep: rumination as a mediator. Stress and Health, 27(2), e71-e82. https://doi.org/10.1002/smi.1337

Betts, S. (2002). An exploration of Multiple Jobholding (moonlighting) and an investigation into the relationship between Multiple Jobholding and Work Related Commitment. Dissertation, Rutgers University.

Blais, A.-R., Thompson, M. M., \& McCreary, D. R. (2009). The development and validation of the Army Post-Deployment Reintegration Scale. Military Psychology, 21(3), 365-386. https://doi.org/10.1080/08995600902914727

Blalock, H. M. (1961). Theory, Measurement, and Replication in the Social Sciences. American Journal of Sociology, 66(4), 342-347. https://doi.org/10.1086/222900 
Combat Experiences, Iso-strain, and Sleep Quality Affect PTSS

Bliese, P. D., \& Castro, C. A. (2000). Role clarity, work overload and organizational support: Multilevel evidence of the importance of support. Work \& Stress, 14(1), 65-73. https://doi.org/10.1080/026783700417230

Bliese, P. D., Thomas, J. L., McGurk, D., McBride, S., \& Castro, C. A. (2011). Mental health advisory teams: a proactive examination of mental health during combat deployments. International Review of Psychiatry (Abingdon, England), 23(2), 127134. https://doi.org/10.3109/09540261.2011.558834

Bliese, P. D., Wright, K. M., Adler, A. B., Cabrera, O., Castro, C. A., \& Hoge, C. W. (2008). Validating the Primary Care Posttraumatic Stress Disorder Screen and the Posttraumatic Stress Disorder Checklist with soldiers returning from combat. Retrieved from http://doi.apa.org/getdoi.cfm?doi=10.1037/0022-006X.76.2.272

Bliese, P. D., Wright, K. M., Adler, A. B., Thomas, J. L., \& Hoge, C. W. (2007). Timing of postcombat mental health assessments. Psychological Services, 4(3), 141-148. https://doi.org/10.1037/1541-1559.4.3.141

Bonanno, G. A. (2012). Uses and abuses of the resilience construct: Loss, trauma, and health-related adversities. Social Science \& Medicine, 74(5), 753-756. https://doi.org/10.1016/j.socscimed.2011.11.022

Bonanno, G. A., Mancini, A. D., Horton, J. L., Powell, T. M., LeardMann, C. A., Boyko, E. J., ... Smith, T. C. (2012). Trajectories of trauma symptoms and resilience in deployed US military service members: prospective cohort study. The British Journal of Psychiatry, 200(4), 317-323.

https://doi.org/10.1192/bjp.bp.111.096552 
Combat Experiences, Iso-strain, and Sleep Quality Affect PTSS

Booth, B., \& Lederer, S. (2012). Military Families in an Era of Persistent Conflict. In J. H. Laurence \& M. D. Matthews (Eds.), The Oxford handbook of military psychology. New York: Oxford University Press.

Borders, A., McAndrew, L. M., Quigley, K. S., \& Chandler, H. K. (2012). Rumination Moderates the Associations Between PTSD and Depressive Symptoms and Risky Behaviors in U. S. Veterans: Rumination and Risky Behaviors. Journal of Traumatic Stress, 25(5), 583-586. https://doi.org/10.1002/jts.21733

Brady, G. P., \& Hammer, L. B. (2013, August). Study of Employment Retention Veterans (SERVe): Improving Reintegration of Oregon National Guard and Reserves in the Workplace. Presented at the 1st Annual Student Research Symposium, Portland State University. Retrieved from http://pdxscholar.library.pdx.edu/studentsymposium/2013/Presentation/8/

Brancu, M., Thompson, N. L., Beckham, J. C., Green, K. T., Calhoun, P. S., Elbogen, E. B., ... Wagner, H. R. (2014). The impact of social support on psychological distress for U.S. Afghanistan/Iraq era veterans with PTSD and other psychiatric diagnoses. Psychiatry Research, 217(1-2), 86-92. https://doi.org/10.1016/j.psychres.2014.02.025

Breslau, N., Lucia, V. C., \& Davis, G. C. (2004). Partial PTSD versus full PTSD: an empirical examination of associated impairment. Psychological Medicine, 34(7), $1205-1214$.

Brewer, M. (2000). Research Design and Issues of Validity. In H. Reis \& C. Judd (Eds.), Handbook of Research Methods in Social and Personality Psychology. 
Combat Experiences, Iso-strain, and Sleep Quality Affect PTSS

Cambridge, UK; New York, NY; Melbourne, Australia: Cambridge University Press.

Brewin, C. R., Andrews, B., \& Valentine, J. D. (2000). Meta-analysis of risk factors for posttraumatic stress disorder in trauma-exposed adults. Journal of Consulting and Clinical Psychology, 68(5), 748-766. https://doi.org/10.1037/0022-006X.68.5.748

Brewin, C. R., \& Holmes, E. A. (2003). Psychological theories of posttraumatic stress disorder. Clinical Psychology Review, 23(3), 339-376.

https://doi.org/10.1016/S0272-7358(03)00033-3

Briggs, B. (2014, June 20). DoD and VA Can't Prove Their PTSD Care is Working, Study Claims [NBC NEWS]. Retrieved from http://www.nbcnews.com/health/health-news/dod-va-cant-prove-their-ptsd-careworking-study-claims-n136371

Britt, T. W., Greene-Shortridge, T., Brink, S., Nguyen, Q., Hoge, C., \& Castro, C. (2008). Percieved Stigma and Barriers to Care for Psychological Treatment: Implications for Reactions to Stressors in Different Contexts. Journal of Social and Clinical Psychology, 27(4), 317-335.

Bronfenbrenner, U. (1986). Family as a context for human development: Research perspectives. Developmental Psychology, 22(6), 723:742.

Brown, D. (2007, January 11). Pentagon changes policy for calling up National Guard, Reserves. McClatchy-Tribune Information Services. Retrieved from Www.mcclatchydc.com/2007/01/11/15487_pentagon-changes-policy-forcalling.html 
Combat Experiences, Iso-strain, and Sleep Quality Affect PTSS

Building Civilian Career Is Challenge for National Guard Members. (2015). San Diego, CA: KPBS. Retrieved from http://www.kpbs.org/news/2015/apr/09/buildingcivilian-career-no-easy-task-national-gua/

Buysse, D. J., Reynolds, C. F., Monk, T. H., Berman, S. R., \& Kupfer, D. J. (1989). The Pittsburgh Sleep Quality Index: a new instrument for psychiatric practice and research. Psychiatry Research, 28(2), 193-213.

Byars-Winston, A., Fouad, N., \& Wen, Y. (2015). Race/ethnicity and sex in U.S. occupations, 1970-2010: Implications for research, practice, and policy. Journal of Vocational Behavior, 87, 54-70. https://doi.org/10.1016/j.jvb.2014.12.003

Cahill, J., \& Landsbergis, P. A. (1996). Job strain among post office mailhandlers. International Journal of Health Services: Planning, Administration, Evaluation, 26(4), 731-750.

Caplan, R. D., Cobb, S., \& French, B., J. (1975). Job demands and worker health. (HEW Pub No). 715-160, Washington, DC.: NIOSH.

Cassel, J. (1976). The contribution of the social environment to host resistance: The Fourth Wade Hampton Frost Lecture. American Journal of Epidemiology, 104(2), $107-123$.

Chambers, J. W., \& Anderson, F. (Eds.). (1999). The Oxford companion to American military history. New York: Oxford University Press.

Chang, C., \& Spector, P. (2011). Cross-Cultural Occupational HealthPsychology. In J. C. Quick \& L. E. Tetrick (Eds.), Occupational Health Psychology (p. 440). Washington, D.C.: American Psychological Association. 
Combat Experiences, Iso-strain, and Sleep Quality Affect PTSS

Chen, C.-H. V., Wang, S.-J., Chang, W.-C., \& Hu, C.-S. (2008). The Effect of LeaderMember Exchange, Trust, Supervisor Support on Organizational Citizenship Behavior in Nurses: Journal of Nursing Research, 16(4), 321-328. https://doi.org/10.1097/01.JNR.0000387319.28010.5e

Chen, S., Westman, M., \& Eden, D. (2009). Impact of enhanced resources on anticipatory stress and adjustment to new information technology: A fieldexperimental test of conservation of resources theory. Journal of Occupational Health Psychology, 14(3), 219-230. https://doi.org/10.1037/a0015282

Cheung, G. W., \& Rensvold, R. B. (2002). Evaluating Goodness-of-Fit Indexes for Testing Measurement Invariance. Structural Equation Modeling: A Multidisciplinary Journal, 9(2), 233-255. https://doi.org/10.1207/S15328007SEM0902_5

Choi, N. G., DiNitto, D. M., \& Marti, C. N. (2015). Alcohol and other substance use, mental health treatment use, and perceived unmet treatment need: Comparison between baby boomers and older adults: Substance Use, Mental Health Treatment, and Age Cohort Comparison. The American Journal on Addictions, 24(4), 299307. https://doi.org/10.1111/ajad.12225

Cohen, J., \& Cohen, J. (Eds.). (2003). Applied multiple regression/correlation analysis for the behavioral sciences (3rd ed). Mahwah, N.J: L. Erlbaum Associates.

Cohen, S., \& Edwards, J. R. (1989). Personality Characteristics as Moderators of the Relationship Between Stress and Disorder. In R. W. J. Neufeld (Ed.), Advances in the Investigation of Psychological Stress (p. 235:83). New York: Wiley. 
Combat Experiences, Iso-strain, and Sleep Quality Affect PTSS

Cohen, S., \& Wills, T. (1985). Stress, social support, and the buffering hypothesis. Psychological Bulletin, 98(2), 310-357.

Coltman, T., Devinney, T. M., Midgley, D. F., \& Venaik, S. (2008). Formative versus reflective measurement models: Two applications of formative measurement. Journal of Business Research, 61(12), 1250-1262. https://doi.org/10.1016/j.jbusres.2008.01.013

Congressional Budget Office. (2012). The Veterans Health Administration's Treatment of PTSD and Traumatic Brain Injury Among Recent Combat Veterans (congressional report No. Pub. No. 4097) (p. 40). Washington, D.C. Retrieved from http://www.cbo.gov/sites/default/files/cbofiles/attachments/02-09-PTSD.pdf

Cook, T. D., \& Campbell, D. T. (1979). Quasi-experimentation: design \& analysis issues for field settings. Boston: Houghton Mifflin.

Corrigan, P. W., Watson, A. C., \& Barr, L. (2006). The Self-Stigma of Mental Illness: Implications for Self-Esteem and Self-Efficacy. Journal of Social and Clinical Psychology, 25(8), 875-884. https://doi.org/10.1521/jscp.2006.25.8.875

Corry, N. (2014). Long-term Course of PTSD Revealed (Psychiatry) (p. 2). Medscape. Retrieved from http://www.medscape.com/viewarticle/829872\#vp_2 Crain, T. L., Hammer, L. B., Bodner, T., Kossek, E. E., Moen, P., Lilienthal, R., \& Buxton, O. M. (2014). Work-family conflict, family-supportive supervisor behaviors (FSSB), and sleep outcomes. Journal of Occupational Health Psychology, 19(2), 155-167. https://doi.org/10.1037/a0036010 
Combat Experiences, Iso-strain, and Sleep Quality Affect PTSS

Crocker, L. M., \& Algina, J. (2008). Introduction to classical and modern test theory. Mason, Ohio: Cengage Learning.

Currie, S. L., Day, A., \& Kelloway, E. K. (2011). Bringing the troops back home: Modeling the postdeployment reintegration experience. Journal of Occupational Health Psychology, 16(1), 38-47. https://doi.org/10.1037/a0021724

Da Costa, J. M. (1851). On irritable heart. The American Journal of Medicine, 11(5), 559-567. https://doi.org/10.1016/0002-9343(51)90038-1

Davenport, L. (1972). Special Report [on the] Employment Problems of the Vietnam Veteran (No. ED 063529 AC 012 625) (p. 18). Washington, D.C.: National Advisory Council on Vocational Education.

De Groat, A. S., \& Crowley, R. P. (2013). Looking Critically at Reintegration of Post 9/11 Era Military Veterans. Retrieved from http://media.wix.com/ugd/e96c7d_71799bdae332483289167b3164d39b47.pdf

Demerouti, E., \& Bakker, A. B. (2011). The Job Demands-Resources model:

Challenges for future research. SA Journal of Industrial Psychology, 37(2). https://doi.org/10.4102/sajip.v37i2.974

Demerouti, E., Bakker, A. B., Nachreiner, F., \& Schaufeli, W. B. (2001). The job demands-resources model of burnout. Journal of Applied Psychology, 86(3), 499512. https://doi.org/10.1037//0021-9010.86.3.499

Department of Defense. (2007). Deployment of Members of the National Guard and Reserve in the Global War on Terrorism. Defense Science Board. 
Combat Experiences, Iso-strain, and Sleep Quality Affect PTSS

Dewa, C. S., \& Lin, E. (2000). Chronic physical illness, psychiatric disorder and disability in the workplace. Social Science \& Medicine (1982), 51(1), 41-50.

Diamantopoulos, A., Riefler, P., \& Roth, K. P. (2008). Advancing formative measurement models. Journal of Business Research, 61(12), 1203-1218. https://doi.org/10.1016/j.jbusres.2008.01.009

Dohrenwend, B. P., Turner, J. B., Turse, N. A., Adams, B. G., Koenen, K. C., \& Marshall, R. (2007). Continuing controversy over the psychological risks of Vietnam for U.S. veterans. Journal of Traumatic Stress, 20(4), 449-465. https://doi.org/10.1002/jts.20296

Dohrenwend, B. P., Yager, T. J., Wall, M. M., \& Adams, B. G. (2013). The Roles of Combat Exposure, Personal Vulnerability, and Involvement in Harm to Civilians or Prisoners in Vietnam-War-Related Posttraumatic Stress Disorder. Clinical Psychological Science, 1(3), 223-238. https://doi.org/10.1177/2167702612469355

Doi, Y. (2005). An epidemiologic review on occupational sleep research among Japanese workers. Industrial Health, 43(1), 3-10.

Dong, Y., \& Peng, C.-Y. J. (2013). Principled missing data methods for researchers. SpringerPlus, 2(1), 222. https://doi.org/10.1186/2193-1801-2-222

Donnellan, M. B., Oswald, F. L., Baird, B. M., \& Lucas, R. E. (2006). The mini-IPIP scales: tiny-yet-effective measures of the Big Five factors of personality. Psychological Assessment, 18(2), 192-203. https://doi.org/10.1037/10403590.18.2.192 
Combat Experiences, Iso-strain, and Sleep Quality Affect PTSS

Draper, N. R., \& Smith, H. (2014). Transformation of the Response Variable. In N. R. Draper \& H. Smith, Applied Regression Analysis (pp. 277-298). Hoboken, NJ, USA: John Wiley \& Sons, Inc. Retrieved from http://doi.wiley.com/10.1002/9781118625590.ch13

Dubner, S. (2008, September 22). Who Serves in the Military Today? [Socioeconomic analyses]. Retrieved from http://freakonomics.com/2008/09/22/who-serves-in-themilitary-today/

Duca, K. (2016). “The Walking Wounded”: Here-and-now Coping Strategies to Ease the Reintegration of American Military Veterans [Academic --NYU]. Retrieved from http://steinhardt.nyu.edu/appsych/opus/issues/2013/spring/duca

Dursa, E. K., Reinhard, M. J., Barth, S. K., \& Schneiderman, A. I. (2014). Prevalence of a Positive Screen for PTSD Among OEF/OIF and OEF/OIF-Era Veterans in a Large Population-Based Cohort: Positive PTSD Screen in OEF/OIF Veterans. Journal of Traumatic Stress, 27(5), 542-549. https://doi.org/10.1002/jts.21956

Dwyer, D. J., \& Ganster, D. C. (1991). The effects of job demands and control on employee attendance and satisfaction. Journal of Organizational Behavior, 12(7), 595-608. https://doi.org/10.1002/job.4030120704

Dwyer, J. E. (1983). Statistical models for the social and behavioral sciences. New York: Oxford Univ. Press.

Earnest, D., Allen, D. G., \& Landis, R. S. (2011). Mechanisms linking realistic job previews with turnover: A meta-analytic path analysis. Personnel Psychology, (64), $865: 897$. 
Combat Experiences, Iso-strain, and Sleep Quality Affect PTSS

Edwards, J. R. (1991). Person-Job fit: A conceptual integration, literature review, and methodological critique. International Review of Industrial and Organizational Psychology, 6, 283:357.

Ehlers, A., \& Clark, D. M. (2000). A cognitive model of posttraumatic stress disorder. Behaviour and Therapy, 38, 319:345.

Eidelson, R., \& Soldz, S. (n.d.). Does Comprehensive Solider Fitness Work? CSF research Fails the Test. Coalition for an Ethical Psychology. Retrieved from www.ethicalpsychology.org

Erbes, C. R., Arbisi, P. A., Kehle, S. M., Ferrier-Auerbach, A. G., Barry, R. A., \& Polusny, M. A. (2011). The distinctiveness of hardiness, positive emotionality, and negative emotionality in National Guard soldiers. Journal of Research in Personality, 45(5), 508-512. https://doi.org/10.1016/j.jrp.2011.07.001

Erbes, C. R., Kaler, M. E., Schult, T., Polusny, M. A., \& Arbisi, P. A. (2011). Mental health diagnosis and occupational functioning in National Guard/Reserve veterans returning from Iraq. Journal of Rehabilitation Research and Development, 48(10), $1159-1170$.

Ertel, K. A., Koenen, K. C., \& Berkman, L. F. (2008). Incorporating Home Demands Into Models of Job Strain: Findings From the Work, Family, and Health Network: Journal of Occupational and Environmental Medicine, 50(11), 1244-1252. https://doi.org/10.1097/JOM.0b013e31818c308d 
Combat Experiences, Iso-strain, and Sleep Quality Affect PTSS

Ferling, J. (2010, January). Myths of the American Revolution. Smithsonian. Retrieved from http://www.smithsonianmag.com/history/myths-of-the-american-revolution$10941835 /$

Flo, E., Pallesen, S., Åkerstedt, T., Magerøy, N., Moen, B. E., Grønli, J., ... Bjorvatn, B. (2013). Shift-related sleep problems vary according to work schedule. Occupational and Environmental Medicine, 70(4), 238-245. https://doi.org/10.1136/oemed-2012-101091

Folkman, S., Lazarus, R. S., Gruen, R. J., \& DeLongis, A. (1986). Appraisal, Coping, Health Status, and Psychological Symptoms, 50(3), 571:579.

Frese, M. (1999). Social Support as a moderator of the relationship between work stressors and psychological dysfunctioning - A long study. Journal of Occupational Health Psychology, 4(3), 179-192.

Friedman, H. S. (Ed.). (2011). The Oxford handbook of health psychology. Oxford: New York: Oxford University Press.

Frone, M. R., Russell, M., \& Cooper, M. L. (1997). Relation of work-family conflict to health outcomes: A four-year longitudinal study of employed parents. Journal of Occupational and Organizational Psychology, 70(4), 325-335. https://doi.org/10.1111/j.2044-8325.1997.tb00652.x

Frone, M. R., Yardley, J. K., \& Markel, K. S. (1997). Developing and Testing an Integrative Model of the Work-Family Interface. Journal of Vocational Behavior, 50(2), 145-167. https://doi.org/10.1006/jvbe.1996.1577 
Combat Experiences, Iso-strain, and Sleep Quality Affect PTSS

Fujita, S. (2014). On the Causes of Declines in the Labor Force Participation Rate (Special Report) (p. 12). Philadelphia: Federal Reserve Bank.

Fulton, J. J., Calhoun, P. S., Wagner, H. R., Schry, A. R., Hair, L. P., Feeling, N., ... Beckham, J. C. (2015). The prevalence of posttraumatic stress disorder in Operation Enduring Freedom/Operation Iraqi Freedom (OEF/OIF) Veterans: A meta-analysis. Journal of Anxiety Disorders, 31, 98-107. https://doi.org/10.1016/j.janxdis.2015.02.003

Ganster, D. (2013). Preventive stress management in organizations. (J. C. Quick, Ed.) (2nd ed). Washington, DC: American Psychological Association.

Ganster, D. C., Fusilier, M. R., \& Mayes, B. T. (1986). Role of social support in the experience of stress at work. The Journal of Applied Psychology, 71(1), 102-110.

GAO. (2014). Military and Veteran Support: DOD and VA Programs That Address the Effects of Combat and Transition to Civilan Life (Report to Congressional Committees No. GAO-15-24) (p. 75). Washington, D.C: United States Government Accountability Office.

Gelman, A. (2013). Understanding posterior p-values. Electronic Journal of Statistics.

Gerhart, J. I., Canetti, D., \& Hobfoll, S. (2015). Traumatic Stress in Overview: Definition, Context, Scope, and Long-Term Outcomes. In press.

Germain, A. (2013). Sleep Disturbances as the Hallmark of PTSD: Where Are We Now? American Journal of Psychiatry, 170(4), 372. https://doi.org/10.1176/appi.ajp.2012.12040432 
Combat Experiences, Iso-strain, and Sleep Quality Affect PTSS

Germain, A., \& Zadra, A. (2009). Dreams and Nightmares in PTSD. In R. Stickgold \& M. P. Walker (Eds.), The neuroscience of sleep. London; Boston: Academic Press/Elsevier.

Gilbertson, M. W., Shenton, M. E., Ciszewski, A., Kasai, K., Lasko, N. B., Orr, S. P., \& Pitman, R. K. (2002). Smaller hippocampal volume predicts pathologic vulnerability to psychological trauma. Nature Neuroscience, 5(11), 1242-1247. https://doi.org/10.1038/nn958

Glass, J., \& Fujimoto, T. (1995). Employer Characteristics and the Provision of Family Responsive Policies. Work and Occupations, 22(4), 380-411. https://doi.org/10.1177/0730888495022004002

Goff, S. J., Mount, M. K., \& Jamison, R. L. (1990). EMPLOYER SUPPORTED CHILD CARE, WORK/ FAMILY CONFLICT, AND ABSENTEEISM: A FIELD STUDY. Personnel Psychology, 43(4), 793-809. https://doi.org/10.1111/j.17446570.1990.tb00683.x

Gold, J., Taft, T., Keehn, M., King, D. W., King, L. A., \& Samper, R. (2007). PTSD symptom severity and family adjustment among female Vietnam veterans. Military Psychology, 19(2), 71-81.

Grandner, M. A., Kripke, D. F., Yoon, I.-Y., \& Youngstedt, S. D. (2006). Criterion validity of the Pittsburgh Sleep Quality Index: Investigation in a non-clinical sample. Sleep and Biological Rhythms, 4(2), 129-136. https://doi.org/10.1111/j.1479-8425.2006.00207.x 
Greguras, G. J. (2009). Different fits satisfy different needs: Linking personenvironment fit to employee commitment and performance using selfdetermination theory. Journal of Applied Psychology, 94, 465:477.

Griffith, J. (2009). Being a Reserve Soldier: A Matter of Social Identity. Armed Forces \& Society, 36(1), 38-64. https://doi.org/10.1177/0095327X08327819

Griffith, J., \& Bryan, C. J. (2016). Suicides in the U.S. Military: Birth Cohort Vulnerability and the All-Volunteer Force. Armed Forces \& Society, 42(3), 483500. https://doi.org/10.1177/0095327X15614552

Griffith, J., \& Vaitkus, M. (2013). Perspectives on Suicide in the Army National Guard. Armed Forces \& Society, 39(4), 628-653. https://doi.org/10.1177/0095327X12471333

Groer, M. W., Kane, B., Williams, S. N., \& Duffy, A. (2015). Relationship of PTSD Symptoms With Combat Exposure, Stress, and Inflammation in American Soldiers. Biological Research for Nursing, 17(3), 303-310. https://doi.org/10.1177/1099800414544949

Gros, D. F., Price, M., Magruder, K. M., \& Frueh, B. C. (2012). Symptom overlap in posttraumatic stress disorder and major depression. Psychiatry Research, 196(2-3), 267-270. https://doi.org/10.1016/j.psychres.2011.10.022

Guyker, W. M., Donnelly, K., Donnelly, J. P., Dunnam, M., Warner, G. C., Kittleson, J., ... Meier, S. T. (2013). Dimensionality, reliability, and validity of the combat experiences scale. Military Medicine, 178(4), 377-384. https://doi.org/10.7205/MILMED-D-12-00223 
Haass, R. (2007, 2008). Supporting US Foreign Policy in the Post-9/11 World: Policymakers and the Intelligence Community. Central Intelligence Agency. Retrieved from https://www.cia.gov/library/center-for-the-study-ofintelligence/csi-publications/csi-studies/studies/vol46no3/article01.html

Halbesleben, J., \& Buckley, M. R. (2004). Burnout in Organizational Life. Journal of Management, 30(6), 859-879. https://doi.org/10.1016/j.jm.2004.06.004

Hamilton, N. A., Gallagher, M. W., Preacher, K. J., Stevens, N., Nelson, C. A., Karlson, C., \& McCurdy, D. (2007). Insomnia and well-being. Journal of Consulting and Clinical Psychology, 75(6), 939-946. https://doi.org/10.1037/0022-006X.75.6.939

Hammer, L. (2013, August). Using Occupational Health Psychology (OHP) to Support Veteran Reintegration into the Civilian Workforce. Presented at the American Psychological Association:121st Annual Convention, Honolulu, HI.

Hammer, L., Kossek, E., Zimmerman, K., \& Daniels, R. (2007). Clarifying the construct of family supportive supervisory behaviors: A multilevel perspective. In P. Perrewe \& D. Ganster (Eds.), Research on Occupational Stress and Well-Being (Vol. 6, p. 165:204). Greenwich, CT: Elsevier Press.

Harrell, M. C., \& Berglass, N. (2012). Employing America's Veterans: Perspectives from Businesses. Washington, D.C.: Center for New American Sercurity.

Harrington, J. M. (2001). Health effects of shift work and extended hours of work. Occupational and Environmental Medicine, 58(1), 68-72. https://doi.org/10.1136/oem.58.1.68 
Combat Experiences, Iso-strain, and Sleep Quality Affect PTSS

Harvard Medical School. (2007, December 18). Healthy Sleep: Why do We Sleep, Anyway? Retrieved from http://healthysleep.med.harvard.edu/healthy/matters/benefits-of-sleep/why-do-wesleep

Harvey, A. G., Jones, C., \& Schmidt, A. (2003). Sleep and posttraumatic stress disorder: a review. Clinical Psychology Review, 23, 377:407.

Heaney, C. (2011). Worksite Health Interventions: Targets for Change and Strategies for Attaining Them. In J. C. Quick \& L. E. Tetrick (Eds.), Handbook of occupational health psychology (2nd ed). Washington, DC: American Psychological Association.

Heinrichs, M., Baumgartner, T., Kirschbaum, C., \& Ehlert, U. (2003). Social support and oxytocin interact to suppress cortisol and subjective responses to psychosocial stress. Biological Psychiatry, 54(12), 1389-1398.

Hirsch, B. T., \& Husain, M.M. (2014). Multiple Job Holding, Local Labor Markets, and the Business Cycle (p. 22). Presented at the Society for Labor Economists (SOLE) 19th Annual Meeting, Arlington, VA.

Hobfoll, S. (1989). Conservation of Resources: A New Attempt at Conceptualizing Stress. American Psychologist, 44(3), 513:524.

Hobfoll, S. E. (1998). Stress, culture, and community: the psychology and philosophy of stress. New York: Plenum Press.

Hobfoll, S. E., Vinokur, A. D., Pierce, P. F., \& Lewandowski-Romps, L. (2012). The combined stress of family life, work, and war in Air Force men and women: A test 
Combat Experiences, Iso-strain, and Sleep Quality Affect PTSS

of conservation of resources theory. International Journal of Stress Management, 19(3), 217-237. https://doi.org/10.1037/a0029247

Hoff, P. D. (2009). A first course in Bayesian statistical methods. London : New York: Springer.

Hoge, C. W., Auchterlonie, J. L., \& Milliken, C. S. (2006). Mental health problems, use of mental health services, and attrition from military service after returning from deployment to Iraq or Afghanistan. JAMA, 295(9), 1023-1032. https://doi.org/10.1001/jama.295.9.1023

Hoit, K. (2012, March 6). The "Dangerous" Veteran: An Inaccurate Media Narrative Takes Hold. Retrieved from http://www.blogs.va.gov/VAntage/6026/the$\% \mathrm{E} 2 \% 80 \% 9 \mathrm{Cdangerous} \% \mathrm{E} 2 \% 80 \% 9 \mathrm{D}$-veteran-an-inaccurate-media-narrativetakes-hold/

Holahan, C. J., Moos, R. H., Holahan, C. K., \& Brennan, P. L. (1997). Social context, coping strategies, and depressive symptoms: an expanded model with cardiac patients. Journal of Personality and Social Psychology, 72(4), 918-928.

Holland, P. W. (1986). Statistics and Causal Inference. Journal of the American Statistical Association, 18(396), 945-960.

Horn, J. L., \& McArdle, J. J. (1992). A practical and theoretical guide to measurement invariance in aging research. Experimental Aging Research, 18(3-4), 117-144. https://doi.org/10.1080/03610739208253916

Houkes, I., Janssen, P. P. M., de Jonge, J., \& Nijhuis, F. J. N. (2001). Specific relationships between work characteristics and intrinsic work motivation, burnout 
Combat Experiences, Iso-strain, and Sleep Quality Affect PTSS

and turnover intention: A multi-sample analysis. European Journal of Work and Organizational Psychology, 10(1), 1-23.

https://doi.org/10.1080/13594320042000007

Howell, D. C. (2013). Statistical methods for psychology (8th ed). Belmont, CA:

Wadsworth Cengage Learning.

Hu, L., \& Bentler, P. M. (1999). Cutoff criteria for fit indexes in covariance structure analysis: Conventional criteria versus new alternatives. Structural Equation Modeling: A Multidisciplinary Journal, 6(1), 1-55.

https://doi.org/10.1080/10705519909540118

Ingram, R. E., \& Luxton, D. D. (2005). Vulnerability-Stress Models. In Development of Psychopathology: A Vulnerability-Stress Perspective (pp. 32-46). 2455 Teller Road, Thousand Oaks California 91320 United States: SAGE Publications, Inc. Retrieved from http://sk.sagepub.com/books/development-ofpsychopathology/n2.xml

Insana, S. P., Hall, M., Buysse, D. J., \& Germain, A. (2013). Validation of the Pittsburgh Sleep Quality Index Addendum for Posttraumatic Stress Disorder (PSQI-A) in U.S. Male Military Veterans: Sleep Disturbances Among Military Veterans. Journal of Traumatic Stress, 26(2), 192-200.

https://doi.org/10.1002/jts.21793

Institute of Medicine (U.S.). (2012). Treatment for posttraumatic stress disorder in military and veteran populations: initial assessment. Washington, D.C: National Academies Press. 
Combat Experiences, Iso-strain, and Sleep Quality Affect PTSS

Institute of Medicine (U.S.) (Ed.). (2013). Returning home from Iraq and Afghanistan: assessment of readjustment needs of veterans, service members, and their families. Washington, D.C: National Academies Press.

Iversen, A. C., Fear, N. T., Ehlers, A., Hacker Hughes, J., Hull, L., Earnshaw, M., ... Hotopf, M. (2008). Risk factors for post-traumatic stress disorder among UK Armed Forces personnel. Psychological Medicine, 38(4), 511-522. https://doi.org/10.1017/S0033291708002778

Izzo, P. (2013, May 25). Number of the Week: More Veterans Survive, but With More Injuries. Retrieved from http://blogs.wsj.com/economics/2013/05/25/number-ofthe-week-more-veterans-survive-but-with-more-injuries/

Jackson, D. L. (2003). Revisiting sample size and number of parameter estimates: some support for the N:q hypothesis. Structural Equation Modeling: A Multidisciplinary Journal, 10(1), 128-141.

Jakšić, N., Brajković, L., Ivezić, E., Topić, R., \& Jakovljević, M. (2012). The role of personality traits in posttraumatic stress disorder (PTSD). Psychiatria Danubina, 24(3), 256-266.

Jakupcak, M., Cook, J., Imel, Z., Fontana, A., Rosenheck, R., \& McFall, M. (2009). Posttraumatic stress disorder as a risk factor for suicidal ideation in Iraq and Afghanistan War veterans. Journal of Traumatic Stress, 22(4), 303-306. https://doi.org/10.1002/jts.20423

Jakupcak, M., Hoerster, K. D., Varra, A., Vannoy, S., Felker, B., \& Hunt, S. (2011). Hopelessness and Suicidal Ideation in Iraq and Afghanistan War Veterans 
Combat Experiences, Iso-strain, and Sleep Quality Affect PTSS

Reporting Subthreshold and Threshold Posttraumatic Stress Disorder: The Journal of Nervous and Mental Disease, 199(4), 272-275.

https://doi.org/10.1097/NMD.0b013e3182124604

Jimmieson, N. L., \& Terry, D. J. (1999). The moderating role of task characteristics in determining responses to a stressful work simulation. Journal of Organizational Behavior, 20, 709:736.

Johnson, J. V., \& Hall, E. M. (1988). Job strain, work place social support, and cardiovascular disease: A cross-sectional study of a random sample of the Swedish working population. American Journal of Public Health, 78(10), 1336:1342.

Jones, E., \& Wessely, S. (2007). A paradigm shift in the conceptualization of psychological trauma in the 20th century. Journal of Anxiety Disorders, 21(2), 164-175. https://doi.org/10.1016/j.janxdis.2006.09.009

Joshi, A. (2013). High Dimensional Data. University of San Francisco. Retrieved from http://cs.usfca.edu/ apjoshi/cs686/lectures/High_Dimensional.pdf

Joyce, J. M. (2004). Bayesianism. In A. R. Mele \& P. Rawling (Eds.), The Oxford Handbook of Rationality. Oxford University Press.

Junger, S. (2015, June). How PTSD Became A Problem Far Beyond The Battlefield. Vanity Fair. Retrieved from http://www.vanityfair.com/news/2015/05/ptsd-warhome-sebastian-junger

Kahn, R. L., \& Antonucci, T. C. (1980). Convoy of Social Support Model. Life-Span Development and Behavior, 3(253). 
Combat Experiences, Iso-strain, and Sleep Quality Affect PTSS

Karasek, R. A. (1979). Job Demands, Job Decision Latitude, and Mental Strain: Implications for Job Redesign. Administrative Science Quarterly, 24(2), 285. https://doi.org/10.2307/2392498

Karasek, R., Brisson, C., Kawakami, N., Houtman, I., Bongers, P., \& Amick, B. (1998). The Job Content Questionnaire (JCQ): an instrument for internationally comparative assessments of psychosocial job characteristics. Journal of Occupational Health Psychology, 3(4), 322-355.

Karasek, R., \& Theorell, T. (1990). Healthy work: Stress, productivity, and the reconstruction of working life. New York, NY: Basic Books.

Keane, T. M., Fairbank, J. A., Caddell, J. M., Zimering, R. T., Taylor, K. L., \& Mora, C. A. (1989). Clinical evaluation of a measure to assess combat exposure. Psychological Assessment: A Journal of Consulting and Clinical Psychology, 1(1), 53-55. https://doi.org/10.1037/1040-3590.1.1.53

Kelloway, E. K., \& Barling, J. (2010). Leadership development as an intervention in occupational health psychology. Work \& Stress, 24(3), 260-279. https://doi.org/10.1080/02678373.2010.518441

Kessler, R. C., Ormel, J., Demler, O., \& Stang, P. E. (2003). Comorbid mental disorders account for the role impairment of commonly occurring chronic physical disorders: results from the National Comorbidity Survey. Journal of Occupational and Environmental Medicine, 45(12), 1257-1266.

https://doi.org/10.1097/01.jom.0000100000.70011.bb 
Combat Experiences, Iso-strain, and Sleep Quality Affect PTSS

Kessler, R. C., Warner, C. H., Ivany, C., Petukhova, M. V., Rose, S., Bromet, E. J., ... Ursano, R. J. (2015). Predicting Suicides After Psychiatric Hospitalization in US Army Soldiers: The Army Study to Assess Risk and Resilience in Servicemembers (Army STARRS). JAMA Psychiatry, 72(1), 49.

https://doi.org/10.1001/jamapsychiatry.2014.1754

Killgore, W. D. S., Cotting, D. I., Thomas, J. L., Cox, A. L., McGurk, D., Vo, A. H., ... Hoge, C. W. (2008). Post-combat invincibility: Violent combat experiences are associated with increased risk-taking propensity following deployment. Journal of Psychiatric Research, 42(13), 1112-1121.

https://doi.org/10.1016/j.jpsychires.2008.01.001

King, D. W., Taft, C., King, L. A., Hammond, C., \& Stone, E. R. (2006). Directionality of the Association Between Social Support and Posttraumatic Stress Disorder: A Longitudinal Investigation. Journal of Applied Social Psychology, 36(12), 29802992. https://doi.org/10.1111/j.0021-9029.2006.00138.x

King, L. A., King, D. W., Fairbank, J. A., Keane, T. M., \& Adams, G. A. (1998). Resilience-recovery factors in post-traumatic stress disorder among female and male Vietnam veterans: hardiness, postwar social support, and additional stressful life events. Journal of Personality and Social Psychology, 74(2), 420-434.

Kirk, M. (2004, October 26). Rumsfeld's War [News documentary]. Boston, Mass.: WGBH Boston.

Kivimäki, M., Nyberg, S. T., Batty, G. D., Fransson, E. I., Heikkilä, K., Alfredsson, L., ... Theorell, T. (2012). Job strain as a risk factor for coronary heart disease: a 
Combat Experiences, Iso-strain, and Sleep Quality Affect PTSS collaborative meta-analysis of individual participant data. The Lancet, 380(9852), 1491-1497. https://doi.org/10.1016/S0140-6736(12)60994-5

Kleykamp, M., \& Hipes, C. (2015). Coverage of Veterans of the Wars in Iraq and Afghanistan in the U.S. Media. Sociological Forum, 30(2), 348-368. https://doi.org/10.1111/socf.12166

Kline, A., Ciccone, D. S., Falca-Dodson, M., Black, C. M., \& Losonczy, M. (2011). Suicidal ideation among National Guard troops deployed to Iraq: the association with postdeployment readjustment problems. The Journal of Nervous and Mental Disease, 199(12), 914-920. https://doi.org/10.1097/NMD.0b013e3182392917

Kline, R. B. (2011). Principles and practice of structural equation modeling (3rd ed). New York: Guilford Press.

Kobasa, S. C. (1979). Stressful life events, personality, and health: an inquiry into hardiness. Journal of Personality and Social Psychology, 37(1), 1-11.

Kobasa, S. C., Maddi, S. R., \& Kahn, S. (1982). Hardiness and health: A prospective study. Journal of Personality and Social Psychology, 42(1), 168-177. https://doi.org/10.1037/0022-3514.42.1.168

Kompier, M. A. J., Taris, T. W., \& van Veldhoven, M. (2012). Tossing and turning-insomnia in relation to occupational stress, rumination, fatigue, and well-being. Scandinavian Journal of Work, Environment \& Health, 38(3), 238-246. https://doi.org/10.5271/sjweh.3263 
Koopman, J., Howe, M., Hollenbeck, J. R., \& Sin, H.-P. (2015). Small sample mediation testing: Misplaced confidence in bootstrapped confidence intervals. Journal of Applied Psychology, 100(1), 194-202. https://doi.org/10.1037/a0036635

Kossek, E. E., \& Nichol, V. (2006). THE EFFECTS OF ON-SITE CHILD CARE ON EMPLOYEE ATTITUDES AND PERFORMANCE. Personnel Psychology, 45(3), 485-509. https://doi.org/10.1111/j.1744-6570.1992.tb00857.x

Kouvonen, A., Oksanen, T., Vahtera, J., Väänänen, A., De Vogli, R., Elovainio, M., ... Kivimäki, M. (2008). Work-place social capital and smoking cessation: the Finnish Public Sector Study. Addiction (Abingdon, England), 103(11), 1857-1865. https://doi.org/10.1111/j.1360-0443.2008.02315.x

Krauss, A. D., Chen, P. Y., DeArmond, S., \& Moorcroft, B. (2005). Sleepiness in the Workplace: Causes, Consequences, and Countermeasures. In C. L. Cooper \& I. T. Robertson (Eds.), International Review of Industrial and Organizational Psychology 2003 (pp. 81-129). Chichester, UK: John Wiley \& Sons, Ltd. Retrieved from http://doi.wiley.com/10.1002/0470013346.ch3

Kukla, M., Rattray, N. A., \& Salyers, M. P. (2015). Mixed methods study examining work reintegration experiences from perspectives of Veterans with mental health disorders. Journal of Rehabilitation Research and Development, 52(4). https://doi.org/10.1682/JRRD.2014.11.0289

Kulka, R. A. (Ed.). (1990). Trauma and the Vietnam War generation: report of findings from the National Vietnam veterans readjustment study. New York: Brunner/Mazel. 
Kulka, R., Schlenger, W., Fairbank, J., Hough, R., Jordan, B. K., Marmar, C. ., \& Weiss, D. (1988). Contractual Report of Findings from the National Vietnam Veterans Readjustment Study - Vol. 1: Executive Summary, Description of Findings, and Technical Appendices (p. 644). Springfield, VA: U.S. Department of Commerce.

Kuper, H., \& Marmot, M. (2003). Job strain, job demands, decision latitude, and risk of coronary heart disease within the Whitehall II study. Journal of Epidemiology \& Community Health, 57(2), 147-153. https://doi.org/10.1136/jech.57.2.147

Lakhani, H., \& Fugita, S. S. (1993). Reserve/Guard retention: Moonlighting or patriotism? Military Psychology, 5(2), 113-125. https://doi.org/10.1207/s15327876mp0502_3

LaMontagne, A. D., Keegel, T., Louie, A. M., \& Ostry, A. (2010). Job stress as a preventable upstream determinant of common mental disorders: A review for practitioners and policy-makers. Advances in Mental Health, 9(1), 17-35. https://doi.org/10.5172/jamh.9.1.17

Landsbergis, Schnall, Belkic, Schwartz, \& Pickering. (2011). In J. C. Quick \& L. E. Tetrick (Eds.), Handbook of occupational health psychology (2nd ed). Washington, DC: American Psychological Association.

Landy, F. J., \& Conte, J. M. (2013). Work in the 21st century: an introduction to industrial and organizational psychology (Fourth edition). Hoboken, NJ: Wiley. Langford, C. P. H., Bowsher, J., \& Lillis, P. P. (1997). Social support: a conceptual analysis. Journal of Advanced Nursing, 25, 95:100. 
Laposa, J. M., Alden, L. E., \& Fullerton, L. M. (2003). Work stress and posttraumatic stress disorder in ED nurses/personnel (CE). Journal of Emergency Nursing, 29(1), 23-28. https://doi.org/10.1067/men.2003.7

la Rocco, J. M., \& Jones, A. P. (1978). Co-worker and leader support as moderators of stress-strain relationships in work situations. Journal of Applied Psychology, 63(5), 629-634. https://doi.org/10.1037/0021-9010.63.5.629

Lazarus, R. S., \& Folkman, S. (1984). Stress, appraisal, and coping (Nachdr.). New York: Springer Publ.

Lerner, D., Adler, D. A., Rogers, W. H., Chang, H., Lapitsky, L., McLaughlin, T., \& Reed, J. (2010). Work performance of employees with depression: the impact of work stressors. American Journal of Health Promotion: AJHP, 24(3), 205-213. https://doi.org/10.4278/ajhp.090313-QUAN-103

Lewin, T. (1987, December 30). Nation's Homeless Veterans Battle a New Foe: Defeatism. The New York Times.

Liberzon, I., \& Abelson, J. L. (2016). Context Processing and the Neurobiology of PostTraumatic Stress Disorder. Neuron, 92(1), 14-30. https://doi.org/10.1016/j.neuron.2016.09.039

Li, C.-Y., \& Sung, F.-C. (1999). A review of the healthy worker effect in occupational epidemiology. Occupational Medicine, 49, 225-229.

Lim, D., Sanderson, K., \& Andrews, G. (2000). Lost productivity among full-time workers with mental disorders. The Journal of Mental Health Policy and Economics, 3(3), 139-146. 
Combat Experiences, Iso-strain, and Sleep Quality Affect PTSS

Lindsay, D. R. (n.d.). Using I-O to Fight a War. Retrieved from http://www.siop.org/tip/july11/05lindsay.aspx

Little, R. (1988). A Test of Missing Completely at Random for Multivariate Data With Missing Values. Journal of the American Statistical Association, 83(404), 1198:1202.

Luchman, J. N., \& González-Morales, M. G. (2013). Demands, control, and support: A meta-analytic review of work characteristics interrelationships. Journal of Occupational Health Psychology, 18(1), 37-52. https://doi.org/10.1037/a0030541

Lunn, D. (2013). The BUGS book: a practical introduction to Bayesian analysis. Boca Raton, FL: CRC Press, Taylor \& Francis Group.

Macera, C. A., Aralis, H. J., Rauh, M. J., \& MacGregor, A. J. (2013). Do Sleep Problems Mediate the Relationship between Traumatic Brain Injury and Development of Mental Health Symptoms after Deployment? SLEEP, 36(1), 83:90. https://doi.org/10.5665/sleep.2306

MacGregor, A., Han, P., \& Galarneau, M. (2011). Effect of dwell time on the mental health of US military personnel with multiple combat tours. American Journal of Public Health.

Macklin, M. L., Metzger, L. J., Litz, B. T., McNally, R. J., Lasko, N. B., Orr, S. P., \& Pitman, R. K. (1998). Lower precombat intelligence is a risk factor for posttraumatic stress disorder. Journal of Consulting and Clinical Psychology, 66(2), 323-326. https://doi.org/10.1037/0022-006X.66.2.323 
Combat Experiences, Iso-strain, and Sleep Quality Affect PTSS

Maddi, S. (1999). Twenty Years of Hardiness Research \& Practice. In 107th Annual Conference (p. 47). Boston, Mass.

Maguen, S., Metzler, T. J., McCaslin, S. E., Inslicht, S. S., Henn-Haase, C., Neylan, T. C., \& Marmar, C. R. (2009). Routine Work Environment Stress and PTSD Symptoms in Police Officers: The Journal of Nervous and Mental Disease, 197(10), 754-760. https://doi.org/10.1097/NMD.0b013e3181b975f8

Mallick, H., \& Nengiun, Y. (2013). Bayesian Methods for High Dimensional Linear Models. Journal of Biometrics \& Biostatistics. https://doi.org/10.4172/21556180.S1-005

Mallinckrodt, B., Abraham, W. T., Wei, M., \& Russell, D. W. (2006). Advances in testing the statistical significance of mediation effects. Journal of Counseling Psychology, 53(3), 372-378. https://doi.org/10.1037/0022-0167.53.3.372

Martz, E., Bodner, T., \& Livneh, H. (2009). Coping as a moderator of disability and psychosocial adaptation among Vietnam theater veterans. Journal of Clinical Psychology, 65(1), 94-112. https://doi.org/10.1002/jclp.20541

Maslach, C. (2003). Job burnout: new directions in research and intervention. Current Directions in Psychological Science, 12(5), 189-192. https://doi.org/10.1111/14678721.01258

Mather, L., Bergström, G., Blom, V., \& Svedberg, P. (2015). High Job Demands, Job Strain, and Iso-Strain Are Risk Factors for Sick Leave due to Mental Disorders: A Prospective Swedish Twin Study With a 5-Year Follow-Up. Journal of 
Combat Experiences, Iso-strain, and Sleep Quality Affect PTSS

Occupational and Environmental Medicine, 57(8), 858-865.

https://doi.org/10.1097/JOM.0000000000000504

McCreary, D. R., Peach, J. M., Blais, A.-R., \& Fikretoglu, D. (2014). Towards a Better Understanding of Post-Deployment Reintegration. In S. MacDermid Wadsworth \& D. S. Riggs (Eds.), Military Deployment and its Consequences for Families (pp. 173-191). New York, NY: Springer New York. Retrieved from http://link.springer.com/10.1007/978-1-4614-8712-8_10

McDonough, D., \& Sperling, G. (2013, April 5). Incentivizing Employers to Hire Veterans through Permanent Tax Credits. The White House.

McNally, R. J. (2007). Revisiting Dohrenwend et al.'s revisit of the National Vietnam Veterans Readjustment Study. Journal of Traumatic Stress, 20(4), 481-486. https://doi.org/10.1002/jts.20257

Milliken, C. S., Auchterlonie, J. L., \& Hoge, C. W. (2007). Longitudinal Assessment of Mental Health Problems Among Active and Reserve Component Soldiers Returning From the Iraq War. JAMA, 298(18), 2141. https://doi.org/10.1001/jama.298.18.2141

Milner, A., Spittal, M. J., Pirkis, J., \& LaMontagne, A. D. (2013). Suicide by occupation: systematic review and meta-analysis. The British Journal of Psychiatry, 203(6), 409-416. https://doi.org/10.1192/bjp.bp.113.128405

Mollayeva, T., Thurairajah, P., Burton, K., Mollayeva, S., Shapiro, C. M., \& Colantonio, A. (2016). The Pittsburgh sleep quality index as a screening tool for sleep dysfunction in clinical and non-clinical samples: A systematic review and 
Combat Experiences, Iso-strain, and Sleep Quality Affect PTSS

meta-analysis. Sleep Medicine Reviews, 25, 52-73.

https://doi.org/10.1016/j.smrv.2015.01.009

Morin, C. M., Belleville, G., Bélanger, L., \& Ivers, H. (2011). The Insomnia Severity Index: psychometric indicators to detect insomnia cases and evaluate treatment response. Sleep, 34(5), 601-608.

Morin, R. (2011). The Difficult Transition from Military to Civilian Life. Pew Research Center. Retrieved from http://www.pewsocialtrends.org/2011/12/08/the-difficulttransition-from-military-to-civilian-life/

Morrison, D. L., Dunne, M. P., Fitzgerald, R., \& Cloghan, D. (1992). Job design and levels of physical and mental strain among Australian prison officers. Work \& Stress, 6(1), 13-31. https://doi.org/10.1080/02678379208257036

Morrow, J. (Ed.). (2011). Army Force Generation. U.S. Department of the Army.

Mount, M. (2004, June 29). Pentagon to call up rarely tapped Reserves. CNN/Washington Bureau. Retrieved from www.cnn.com/2004/US/06/29/pentagon.reserves

Mowrer, O. H. (1960). Learning Theory and the Symbolic Process. Wiley \& Sons. Mullen, J., \& Kelloway, E. K. (2011). Occupational health and safety leadership. (J. Campbell, Quick, \& L. E. Tetrick, Eds.) (2nd ed.). Washington, D.C: American Psychological Association.

Mulrine, A. (2012, March 23). Sgt. Robert Bales and multiple tours of duty: How many is too many? The Christian Science Monitor. Washington, D.C. 
Combat Experiences, Iso-strain, and Sleep Quality Affect PTSS

Muthen, B., \& Asparouhov, T. (2011). Bayesian SEM: A more flexible representation of substantive theory. California: USA. Retrieved from https://www.statmodel.com/download/BSEMv4REVISED

Muthén, B., \& Asparouhov, T. (2012a). Bayesian structural equation modeling: A more flexible representation of substantive theory. Psychological Methods, 17(3), 313335. https://doi.org/10.1037/a0026802

Muthén, B., \& Asparouhov, T. (2012b). Bayesian structural equation modeling: A more flexible representation of substantive theory. Psychological Methods, 17(3), 313335. https://doi.org/10.1037/a0026802

Muthen, B., \& Muthen, L. (1998). Mplus User's Guide (6th ed.). Los Angeles, CA: Muthen \& Muthen.

Mysliwiec, V., Gill, J., Lee, H., Baxter, T., Pierce, R., Barr, T. L., ... Roth, B. J. (2013). Sleep Disorders in US Military Personnel. Chest, 144(2), 549-557. https://doi.org/10.1378/chest.13-0088

Mysliwiec, V., McGraw, L., Pierce, R., Smith, P., Trapp, B., \& Roth, B. J. (2013). Sleep disorders and associated medical comorbidities in active duty military personnel. Sleep, 36(2), 167-174. https://doi.org/10.5665/sleep.2364

Nahum-Shani, I., \& Bamberger, P. A. (2011). Explaining the variable effects of social support on work-based stressor-strain relations: The role of perceived pattern of support exchange. Organizational Behavior and Human Decision Processes, 114(1), 49-63. https://doi.org/10.1016/j.obhdp.2010.09.002 
Combat Experiences, Iso-strain, and Sleep Quality Affect PTSS

National Bureau of Economic Research. (2010). Business Cycle Dating Committee. Cambridge, Mass. Retrieved from http://www.nber.org/cycles/sept2010.html National Human Genome Research Institute. (2016). About NHGRI: A Brief Historty and Timeline. Retrieved from https://www.genome.gov/10001763/about-nhgri-abrief-history-and-timeline/

Neff, W. S. (1985). Work and Human Behavior (3rd ed.). Walter De Gruyter, Inc.

Nelson, D. ., \& Simmons, B. . (2011). Savoring Eustress While Coping With Distress: The Holistic Model of Stress. In J. C. Quick \& L. Tetrick (Eds.), Occupational Health Psychology (2nd Edition, p. 440). Washington, D.C: American Psychological Association.

Nieuwenhuijsen, K., Bruinvels, D., \& Frings-Dresen, M. (2010). Psychosocial work environment and stress-related disorders, a systematic review. Occupational Medicine, 60(4), 277-286. https://doi.org/10.1093/occmed/kqq081

NIOSH. (2016, April). National occupational research agenda (NORA/national Total Worker Health research, practice, policy, and capacity. U.S. Department of Health and Human Services, Centers for Disease Control and Prevention, National Institute for Occupational Safety and Health.

Noe, R. A., \& Ford, J. K. (1992). Emerging issues and new directions for training research. Research in Personel and Human Resources Management, 10, 345-384.

Odle-Dusseau, H. N., Herleman, H. A., Britt, T. W., Moore, D. D., Castro, C. A., \& McGurk, D. (2013). Family-supportive work environments and psychological 
Combat Experiences, Iso-strain, and Sleep Quality Affect PTSS strain: A longitudinal test of two theories. Journal of Occupational Health Psychology, 18(1), 27-36. https://doi.org/10.1037/a0030803

O’Driscoll, M. P., Poelmans, S., Spector, P. E., Kalliath, T., Allen, T. D., Cooper, C. L., \& Sanchez, J. I. (2003). Family-responsive interventions, perceived organizational and supervisor support, work-family conflict, and psychological strain. International Journal of Stress Management, 10(4), 326-344. https://doi.org/10.1037/1072-5245.10.4.326

Office of the Chairman of the Joint Chiefs of Staff. (2014). Veteran Stereotypes: A Closer Look (p. 20). Washington, D.C.

Oksanen, T., Kawachi, I., Kouvonen, A., Takao, S., Suzuki, E., Virtanen, M., ... Vahtera, J. (2013). Workplace Determinants of Social Capital: Cross-Sectional and Longitudinal Evidence from a Finnish Cohort Study. PLoS ONE, 8(6), e65846. https://doi.org/10.1371/journal.pone.0065846

Oksanen, T., Kouvonen, A., Kivimäki, M., Pentti, J., Virtanen, M., Linna, A., \& Vahtera, J. (2008). Social capital at work as a predictor of employee health: multilevel evidence from work units in Finland. Social Science \& Medicine (1982), 66(3), 637-649. https://doi.org/10.1016/j.socscimed.2007.10.013

Olff, M., Frijling, J. L., Kubzansky, L. D., Bradley, B., Ellenbogen, M. A., Cardoso, C., ... van Zuiden, M. (2013). The role of oxytocin in social bonding, stress regulation and mental health: An update on the moderating effects of context and interindividual differences. Psychoneuroendocrinology, 38(9), 1883-1894. https://doi.org/10.1016/j.psyneuen.2013.06.019 
Combat Experiences, Iso-strain, and Sleep Quality Affect PTSS

Olff, M., Koch, S. B. J., Nawijn, L., Frijling, J. L., Van Zuiden, M., \& Veltman, D. J. (2014). Social support, oxytocin, and PTSD. European Journal of Psychotraumatology, 5, 26513.

O’Neill, J. W., Harrison, M. M., Cleveland, J., Almeida, D., Stawski, R., \& Crouter, A. C. (2009). Work-family climate, organizational commitment, and turnover: Multilevel contagion effects of leaders. Journal of Vocational Behavior, 74(1), 1829. https://doi.org/10.1016/j.jvb.2008.10.004

Overman, S., \& Leonard, B. (2010). Employing Military Personnel and Recruiting Veterans: What HR can Do (p. 21). Society for Human Resource Management.

Parkes, K. R., Mendham, C. A., \& von Rabenau. (1994). Social Support and the Demand-Discretion Model of Job Stress: Test of Additive and Interactive Effects. Journal of Vocational Behavior, 44, 91:113.

Perl, L. (2015). Veterans and Homelessness (Congressional Research Report). Washington, D.C.: Congressional Research Service.

Perrewe, P. L., \& Ganster, D. C. (2007). Exploring the work and non-work interface. Amsterdam; Oxford: Elsevier JAI. Retrieved from http://public.eblib.com/choice/publicfullrecord.aspx?p=648457

Pietrzak, R. H., Goldstein, M. B., Malley, J. C., Johnson, D. C., \& Southwick, S. M. (2009). Subsyndromal posttraumatic stress disorder is associated with health and psychosocial difficulties in veterans of Operations Enduring Freedom and Iraqi Freedom. Depression and Anxiety, 26(8), 739-744.

https://doi.org/10.1002/da.20574 
Pietrzak, R. H., Johnson, D. C., Goldstein, M. B., Malley, J. C., Rivers, A. J., Morgan, C. A., \& Southwick, S. M. (2010). Psychosocial buffers of traumatic stress, depressive symptoms, and psychosocial difficulties in veterans of Operations Enduring Freedom and Iraqi Freedom: The role of resilience, unit support, and postdeployment social support. Journal of Affective Disorders, 120(1-3), 188-192. https://doi.org/10.1016/j.jad.2009.04.015

Ployhart, R. E., \& Moliterno, T. P. (2011). Emergence of the Human Capital Resource: A Multilevel Model. Academy of Management Review, 36(1), 127-150. https://doi.org/10.5465/amr.2009.0318

Prudential. (2012). Veterans' Employment Challenges: Perceptions and experiences of transitioning from military to civilian life.

Quick, J. C. (1999). Occupational health psychology: historical roots and future directions. Health Psychology: Official Journal of the Division of Health Psychology, American Psychological Association, 18(1), 82-88.

Quick, J. C., Quick, J. D., Nelson, D. L., \& Hurrell, J. J. (1997). Preventive stress management for healthy organizations. In J. C. Quick, J. D. Quick, D. L. Nelson, \& J. J. Hurrell, Preventive stress management in organizations. (pp. 277-300). Washington: American Psychological Association. Retrieved from http://content.apa.org/books/10238-014

Quick, J. C., Tetrick, L. E., \& American Psychological Association (Eds.). (2011). Handbook of occupational health psychology (2nd ed). Washington, DC: American Psychological Association. 
Combat Experiences, Iso-strain, and Sleep Quality Affect PTSS

Raabe, P. H. (1990). The Organizational Effects of Workplace Family Policies Past Weaknesses and Recent Progress Toward Improved Research. Journal of Family Issues, 11(4), 477-491.

Raskind, M. A., Peskind, E. R., Hoff, D. J., Hart, K. L., Holmes, H. A., Warren, D., ... McFall, M. E. (2007). A Parallel Group Placebo Controlled Study of Prazosin for Trauma Nightmares and Sleep Disturbance in Combat Veterans with PostTraumatic Stress Disorder. Biological Psychiatry, 61(8), 928-934. https://doi.org/10.1016/j.biopsych.2006.06.032

Resch, J. P. (1999). Suffering soldiers: Revolutionary War veterans, moral sentiment, and political culture in the early republic. Amherst: University of Massachusetts Press.

Reyes, G. (2008). The encyclopedia of psychological trauma. (J. D. Elhai \& J. D. Ford, Eds.). Hoboken, N.J: Wiley.

Rietschlin, J. (1998, December). Voluntary association membership and psychological distress.

Rissling, M., \& Ancoli-Israel, S. (2009). Sleep in Aging. In R. Stickgold \& M. P. Walker (Eds.), The neuroscience of sleep. London ; Boston: Academic Press/Elsevier.

Riviere, L. A., Kendall-Robbins, A., McGurk, D., Castro, C. A., \& Hoge, C. W. (2011). Coming home may hurt: risk factors for mental ill health in US reservists after deployment in Iraq. The British Journal of Psychiatry, 198(2), 136-142. https://doi.org/10.1192/bjp.bp.110.084863 
Combat Experiences, Iso-strain, and Sleep Quality Affect PTSS

Rosseel, Y. (2015). Package "lavaan.” CRAN.

Ruesch, J. M.D., Jacobson, A. M.D., \& Loeb, M. B.A. (1949). Acculturation and Illness. Psychological Monographs: General and Applied, 62.

Ruh, D., Spicer, P., \& Vaughan, K. (2009). Helping Veterans with Disabilties Transition to Employment. Journal of Postsecondary Education and Disability, 22(1), 67-74.

Rydstedt, L. W., \& Devereux, J. J. (2013). Psychosocial job strain and sleep quality interaction leading to insufficient recovery. International Journal of Environmental Research and Public Health, 10(11), 5863-5873.

https://doi.org/10.3390/ijerph10115863

Sapolsky, R. M. (1983). Endocrine aspects of social instability in the olive baboon (Papio anubis). American Journal of Primatology, 5(4), 365-379.

https://doi.org/10.1002/ajp.1350050406

Sapolsky, R. M. (2002). Chickens, eggs and hippocampal atrophy. Nature Neuroscience, 5(11), 1111-1113. https://doi.org/10.1038/nn1102-1111

Sapolsky, R. M. (2004). Why zebras don't get ulcers / Robert M. Sapolsky (3rd ed). New York: Times Books.

Sargent, L. D., \& Terry, D. J. (1998). The effects of work control and job demands on employee adjustment and work performance. Journal of Occupational and Organizational Psychology, 71(3), 219-236. https://doi.org/10.1111/j.20448325.1998.tb00674.x 
Savoca, E., \& Rosenheck, R. (2000). The civilian labor market experiences of Vietnamera veterans: the influence of psychiatric disorders. The Journal of Mental Health Policy and Economics, 3(4), 199-207. https://doi.org/10.1002/mhp.102

Sayer, N. A., Carlson, K. F., \& Frazier, P. A. (2014). Reintegration Challenges in U.S. Service Members and Veterans Following Combat Deployment: Postdeployment Reintegration Challenges. Social Issues and Policy Review, 8(1), 33-73. https://doi.org/10.1111/sipr.12001

Schaufeli, W. B., \& Taris, T. W. (2014). A Critical Review of the Job DemandsResources Model: Implications for Improving Work and Health. In G. F. Bauer \& O. Hämmig, Bridging Occupational, Organizational and Public Health (pp. 4368). Dordrecht: Springer Netherlands. Retrieved from http://link.springer.com/10.1007/978-94-007-5640-3_4

Schlenger, W. E., Corry, N. H., Kulka, R. A., Williams, C. S., Henn-Haase, C., \& Marmar, C. R. (2015). Design and methods of the national Vietnam veterans longitudinal study. International Journal of Methods in Psychiatric Research, 24(3), 186-203. https://doi.org/10.1002/mpr.1469

Schlenger, W. E., Corry, N. H., Williams, C. S., Kulka, R. A., Mulvaney-Day, N., DeBakey, S., ... Marmar, C. R. (2015). A Prospective Study of Mortality and Trauma-Related Risk Factors Among a Nationally Representative Sample of Vietnam Veterans. American Journal of Epidemiology, kwv217. https://doi.org/10.1093/aje/kwv217 
Schmidt, T. (2011). Overview of Statistical and Research Design in the Social Sciences. Portland State University.

Schultz, I. Z., Chlebak, C. M., \& Stewart, A. M. (2016). Impairment, Disability, and Return to Work. In I. Z. Schultz \& R. J. Gatchel (Eds.), Handbook of Return to Work (Vol. 1, pp. 3-25). Boston, MA: Springer US. Retrieved from http://link.springer.com/10.1007/978-1-4899-7627-7_1

Schwarz, N. (1990). Feeling as Information. In E. T. Higgins \& R. M. Sorrentino (Eds.), Handbook of Motivation \& Cognition: Foundations of Social Behavior. New York \& London: The Guilford Press.

Schwarz, N., \& Clore, G. (1983). Mood, Misattribution, and Judgments of Well-Being: Informative and Directive Functions of Affective States, 45(3), 513:523.

Seal, K. H., Metzler, T. J., Gima, K. S., Bertenthal, D., Maguen, S., \& Marmar, C. R. (2009). Trends and risk factors for mental health diagnoses among Iraq and Afghanistan veterans using Department of Veterans Affairs health care, 2002-2008. American Journal of Public Health, 99(9), 1651-1658. https://doi.org/10.2105/AJPH.2008.150284

Selye, H. (1950). Stress and the General Adaptation Syndrome. British Medical Journal. Retrieved from https://www.ncbi.nlm.nih.gov/pmc/articles/PMC2038162/pdf/brmedj036030003.pdf 
Combat Experiences, Iso-strain, and Sleep Quality Affect PTSS

Seo, M.-G., Barrett, L. F., \& Bartunek, J. M. (2004). THE ROLE OF AFFECTIVE EXPERIENCE IN WORK MOTIVATION. Academy of Management Review. Academy of Management, 29(3), 423-439.

Shapiro, S. S., \& Wilk, M. B. (1965). An Analysis of Variance Test for Normality (Complete Samples). Biometrika, 52(3/4), 591. https://doi.org/10.2307/2333709

Shea, M. T., Reddy, M. K., Tyrka, A. R., \& Sevin, E. (2013). Risk factors for postdeployment posttraumatic stress disorder in national guard/reserve service members. Psychiatry Research, 210(3), 1042-1048. https://doi.org/10.1016/j.psychres.2013.08.039

Shea, M. T., Vujanovic, A., Mansfield, A., Sevin, E., \& Liu, F. (2010). Posttraumatic Stress Disorder symptoms and functional impairment among OEF an OIF National Guard and Reserve Veterans. Journal of Traumatic Stress, 23(1), 100:107.

Shen, Y.-C., Cunha, J. M., \& Williams, T. V. (2016). Time-varying associations of suicide with deployments, mental health conditions, and stressful life events among current and former US military personnel: a retrospective multivariate analysis. The Lancet. Psychiatry, 3(11), 1039-1048. https://doi.org/10.1016/S22150366(16)30304-2

Shimizu, T., Eto, R., Horiguchi, I., Obata, Y., Feng, Q., \& Nagata, S. (2005). Relationship between turnover and periodic health check-up data among Japanese hospital nurses: a three-year follow-up study. Journal of Occupational Health, 47(4), 327-333. 
Combat Experiences, Iso-strain, and Sleep Quality Affect PTSS

Siegrist, J. (1996). Adverse health effects of high-effort/low-reward conditions. Journal of Occupational Health Psychology, 1(1), 27-41.

Sinclair, R. R., \& Cheung, J. H. (2016). Money Matters: Recommendations for Financial Stress Research in Occupational Health Psychology. Stress and Health: Journal of the International Society for the Investigation of Stress, 32(3), 181-193. https://doi.org/10.1002/smi.2688

Sliter, M. T., \& Boyd, E. M. (2014, October). Two (or three) is not equal to one: Multiple jobholding as a neglected topic in organizational research: MULTIPLE JOBHOLDING. Retrieved from http://doi.wiley.com/10.1002/job.1944

Smid, G. E., Mooren, T. T. M., van der Mast, R. C., Gersons, B. P. R., \& Kleber, R. J. (2009). Delayed Posttraumatic Stress Disorder: Systematic Review, Meta-Analysis, and Meta-Regression Analysis of Prospective Studies. The Journal of Clinical Psychiatry, 70(11), 1572-1582. https://doi.org/10.4088/JCP.08r04484

Social Capital. (2013). [Public Health]. Retrieved from https://www.cdc.gov/healthyplaces/healthtopics/social.htm

Solomon, Z., Horesh, D., \& Ein-Dor, T. (2009). The longitudinal course of posttraumatic stress disorder symptom clusters among war veterans. The Journal of Clinical Psychiatry, 70(6), 837-843.

Solomon, Z., \& Mikulincer, M. (2006). Trajectories of PTSD: a 20-year longitudinal study. The American Journal of Psychiatry, 163(4), 659-666. https://doi.org/10.1176/appi.ajp.163.4.659 
Sonnentag, S., \& Frtiz, C. (2011). Recovery from Wok. In Handbook of Industrial, Work \& Organizational Psychology (pp. S72-S103). Sage Publications.

Spector, P. (1987). Interactive effects of perceived control and job stressors on affective reactions and health outcomes for clerical workers. Work \& Stress, 1(2), 155:162.

Spector, P. E. (2006). Industrial and organizational psychology: research and practice (4th ed). Hoboken, NJ: J. Wiley \& Sons.

Spector, P. E., \& Jex, S. M. (1998). Development of four self-report measures of job stressors and strain: Interpersonal Conflict at Work Scale, Organizational Constraints Scale, Quantitative Workload Inventory, and Physical Symptoms Inventory. Journal of Occupational Health Psychology, 3(4), 356-367.

Stansfeld, S. A., Shipley, M. J., Head, J., \& Fuhrer, R. (2012). Repeated Job Strain and the Risk of Depression: Longitudinal Analyses From the Whitehall II Study. American Journal of Public Health, 102(12), 2360-2366.

https://doi.org/10.2105/AJPH.2011.300589

Stein, L. (1976). How to start a CME program on your hospital or medical society. IMJ. Illinois Medical Journal, 150(1), 45-48.

Sterling, P., \& Eyer, J. (1988). A new paradigm to explain arousal pathology. (S. Fisher \& J. Reason, Eds.). Chester, UK: John Wiley \& Sons.

Stix, G. (2011). The neuroscience of true grit: When tragedy strikes, most of us ultimately rebound surprisingly well. Where does such resilience come from? Scientific American, 304(3), 29:33. 
Stone, C., \& Stone, D. L. (2015). Factors affecting hiring decisions about veterans. Human Resource Management Review, 25(1), 68-79. https://doi.org/10.1016/j.hrmr.2014.06.003

Tabachnick, B. G., \& Fidell, L. S. (2007). Using multivariate statistics (5th ed). Boston: Pearson/Allyn \& Bacon.

Taber, K. H., \& Hurley, R. A. (2009). PTSD and combat-related injuries: functional neuroanatomy. The Journal of Neuropsychiatry and Clinical Neurosciences, 21(1), $1 \mathrm{p}$ preceding 1, 1-4. https://doi.org/10.1176/appi.neuropsych.21.1.iv

Tanielian, T. (2009). Assessing Combat Exposure and Posttraumatic Stress Disorder in Troops and Estimating the Costs to Society. Santa Monica, CA: RAND.

Tanielian, T. L., \& Jaycox, L. (2008). Invisible wounds of war: psychological and cognitive injuries, their consequences, and services to assist recovery. Santa Monica, CA: RAND.

Tanielian, T., \& Ramchand, R. (2015, June 4). The Long Tail of War: War's impact on mental health stays with soldiers long after they leave Iraq and Afghanistan. U.S. News \& World Report. Retrieved from http://www.usnews.com/opinion/blogs/policy-dose/2015/06/04/veterans-battlemental-health-issues-after-iraq-and-afghanistan

Tayeb, M. (1994). Organizations and National Culture: Methodology Considered. Organization Studies, 15(3), 429-445. https://doi.org/10.1177/017084069401500306 
Taylor, P. (2011). The military-civilian gap: War \& sacrifice in the post-9/11 era (p. 151). Pew Social \& Demographic Trends. Retrieved from wWw.pewsocialtrends.org/files/2011/10/veterans-report.pdf

Taylor, S. (2010). Advanced social psychology: the state of the science. (R. F. Baumeister \& E. J. Finkel, Eds.). Oxford; New York: Oxford University Press.

Taylor, S., Dickerson, S. S., \& Klein, L. C. (2002). Toward a biology of social support. (C. R. Snyder \& S. J. Lopez, Eds.).

Taylor, S. E. (2010). In R. F. Baumeister \& E. J. Finkel (Eds.), Health Psychology. Oxford; New York: Oxford University Press.

Taylor, S. E. (2011). Health psychology (8th ed). New York, NY: McGraw-Hill.

Teachman, J. (2004). Military Service during the Vietnam Era: Were There Consequences for Subsequent Civilian Earnings? Social Forces, 83(2), 709-730. https://doi.org/10.1353/sof.2005.0021

Theorell, T., Leymann, H., Jodko, M., Konarski, K., Norbeck, H. E., \& Eneroth, P. (1992). "Person under train" incidents: medical consequences for subway drivers. Psychosomatic Medicine, 54(4), 480-488.

Thomas, L. T., \& Ganster, D. C. (1995). Impact of family-supportive work variables on work-family conflict and strain: A control perspective. Journal of Applied Psychology, 80(1), 6-15. https://doi.org/10.1037/0021-9010.80.1.6

Thompson, C. A., \& Prottas, D. J. (2006). Relationships among organizational family support, job autonomy, perceived control, and employee well-being. Journal of 
Combat Experiences, Iso-strain, and Sleep Quality Affect PTSS

Occupational Health Psychology, 11(1), 100-118. https://doi.org/10.1037/10768998.10.4.100

Thompson, C., Beauvais, L., \& Lyness, K. (1999). When work-family benefits are not enough: The influences of work-family culture on benefit utilization, organizational attachment, and work-family conflict. Journal of Vocational Behavior, 54, 329-415.

Thorndike, R. L. (1949). Personnel selection: Test and measurement techniques. New York, NY: Wiley \& Sons.

Troxel, W., Shih, Pedersen, E., Geyer, L., Fisher, M., Griffin, B., ... Steinberg, P. (2015). Sleep in the military: promoting healthy sleep among U.S. servicemembers. Santa Monica, CA: RAND Corporation.

Tsai, J., \& Rosenheck, R. A. (2015). Risk factors for homelessness among US veterans. Epidemiologic Reviews, 37, 177-195. https://doi.org/10.1093/epirev/mxu004

Tukey, J. W. (n.d.). Exploratory Data Analysis. Reading, Mass: Addison-Wesley Pub. Co.

Ullman, J. B. (2006). Structural equation modeling: reviewing the basics and moving forward. Journal of Personality Assessment, 87(1), 35-50. https://doi.org/10.1207/s15327752jpa8701_03

U.S. Bureau of Labor, Division of Labor Statistics. (2017). Gulf War II \& Civilian Labor Force Participation Rate.

U.S. Census Bureau. (2016). Historical Poverty Tables: People and Families (1959 to 2015). Washington, D.C.: U.S. Census Bureau. Retrieved from 
Combat Experiences, Iso-strain, and Sleep Quality Affect PTSS

http://www.census.gov/data/tables/time-series/demo/income-poverty/historicalpoverty-people.html

U.S. Department of Defense. (2016). Selected Reserves by Rank/Grade (p. 1).

U.S. Department of Defense, National Guard Bureau. (2012). National Guard Posture Statement: Security America Can Afford. Washington, D.C. Retrieved from http://www.nationalguard.mil/portals/31/Documents/PostureStatements/2012\%20N ational\%20Guard\%20Bureau\%20Posture\%20Statement.pdf

U.S. Department of Defense, National Guard Bureau. (2013). National Guard Posture Statement: Security America Can Afford. Washington, D.C. Retrieved from www.nationalguard.mil/features/ngps

U.S. Department of Defense, National Guard Bureau. (2015). National Guard Posture Statement: Security America Can Afford. Washington, D.C. Retrieved from www.nationalguard.mil/features/ngps

U.S. Department of Labor. (2015). Table A-5. Employment status of the civilian population 18 years and over by veteran status, period of service, and sex, not seasonally adjusted (Economic News Release). Bureau of Labor Statistics. Retrieved from http://www.bls.gov/news.release/empsit.t05.htm

U.S. Department of Veterans Affairs. (2014, November). America's Wars - Fact Sheet. Office of Public Affairs Media Relations. Retrieved from www.va.gov/opa/publications/factsheets/fs_americas_wars.pdf

U.S. Department of Veterans Affairs. (2015). 2015 Veterans Economic Opportunity Report (p. 99). Washington, D.C. Retrieved from 
Combat Experiences, Iso-strain, and Sleep Quality Affect PTSS

http://www.benefits.va.gov/benefits/docs/VeteranEconomicOpportunityReport201 5.PDF

U.S. Government Printing Office. (2013). Lowering the Rate of Unemployment for the National Guard and Reserve: Are We Making Process? (Transcript No. Serial No. 113-0). U.S. House of Representatives.

Uvnas-Moberg, K., \& Petersson, M. (2005). [Oxytocin, a mediator of anti-stress, wellbeing, social interaction, growth and healing]. Zeitschrift Für Psychosomatische Medizin Und Psychotherapie, 51(1), 57-80.

Vance, R. (2006). Employee Engagement and Commitment: A guide to understanding and increasing engagement in your organization. Society for Human Resource Management. Retrieved from https://www.shrm.org/about/foundation/research/Documents/1006EmployeeEngag ementOnlineReport.pdf

Van der Doef, M., \& Maes, S. (1999). The Job Demand-Control(-Support) Model and psychological well-being: A review of 20 years of empirical research. Work \& Stress, 13(2), 87:114.

van Liempt, S. (2012). Sleep disturbances and PTSD: a perpetual circle? European Journal of Psychotraumatology, 3(0). https://doi.org/10.3402/ejpt.v3i0.19142

van Liempt, S., van Zuiden, M., Westenberg, H., Super, A., \& Vermetten, E. (2013). IMPACT OF IMPAIRED SLEEP ON THE DEVELOPMENT OF PTSD SYMPTOMS IN COMBAT VETERANS: A PROSPECTIVE LONGITUDINAL COHORT STUDY: Research Article: Impact of Sleep on PTSD Symptom 
Combat Experiences, Iso-strain, and Sleep Quality Affect PTSS

Development. Depression and Anxiety, 30(5), 469-474.

https://doi.org/10.1002/da.22054

Van Til, L., Fikretoglu, D., Pranger, T., Patten, S., Wang, J., Wong, M., ... Pedlar, D. (2013). Work reintegration for veterans with mental disorders: a systematic literature review to inform research. Physical Therapy, 93(9), 1163-1174. https://doi.org/10.2522/ptj.20120156

van Veldhoven, M., Taris, T. W., de Jonge, J., \& Broersen, S. (2005). The Relationship Between Work Characteristics and Employee Health and Well-Being: How Much Complexity Do We Really Need? International Journal of Stress Management, 12(1), 3-28. https://doi.org/10.1037/1072-5245.12.1.3

Vick, B., \& Fontanella, G. (2017). Gender, race \&amp; the veteran wage gap. Social Science Research, 61, 11-28. https://doi.org/10.1016/j.ssresearch.2016.07.005

Vigneaud, V. du, Ressler, C., Swan, J. M., Roberts, C. W., \& Katsoyannis, P. G. (1954). The Synthesis of Oxytocin ${ }^{1}$. Journal of the American Chemical Society, 76(12), 3115-3121. https://doi.org/10.1021/ja01641a004

Vinokur, A. D., Pierce, P. F., \& Lewandowski-Romps, L. (2009). Disentangling the relationships between job burnout and perceived health in a military sample. Stress and Health, 25(4), 355-363. https://doi.org/10.1002/smi.1264

Vinokur, A. D., Pierce, P. F., Lewandowski-Romps, L., Hobfoll, S. E., \& Galea, S. (2011). Effects of war exposure on air force personnel's mental health, job burnout and other organizational related outcomes. Journal of Occupational Health Psychology, 16(1), 3-17. https://doi.org/10.1037/a0021617 
Combat Experiences, Iso-strain, and Sleep Quality Affect PTSS

Wall, T. D., Jackson, P. R., Mullarkey, S., \& Parker, S. K. (1996). The demands-control model of job strain: A more specific test. Journal of Occupational and Organizational Psychology, 69(2), 153-166. https://doi.org/10.1111/j.20448325.1996.tb00607.x

Wang, M.-J., Mykletun, A., Møyner, E. I., Øverland, S., Henderson, M., Stansfeld, S., ... Harvey, S. B. (2014). Job Strain, Health and Sickness Absence: Results from the Hordaland Health Study. PLoS ONE, 9(4), e96025. https://doi.org/10.1371/journal.pone.0096025

Warr, P. B. (1987). Work, unemployment, and mental health. Oxford : New York: Clarendon Press ; Oxford University Press.

Warr, P. B. (2007). Work, happiness, and unhappiness. Mahwah, N.J: Lawrence Erlbaum Associates.

Washington Post, \& Kaiser Family Foundation. (2013, December 15). After the Wars The Washington Post/Kaiser Family Foundation Survey of Iraq and Afghanistan Active Duty Soldiers and Veterans. Washington Post. Washington, D.C.

Watkins, S., \& Sherk, J. (2008). Who Serves in the U.S. Military? The Demographics of Enlisted Troops and Officers (No. CDA08-05). Washington, D.C.

Watson, D., \& Casillas, A. (2003). Neuroticism: Adaptive and maladaptive features. In E. C. Chang \& L. J. Sanna (Eds.), Virtue, vice, and personality: The complexity of behavior. (pp. 145-161). Washington: American Psychological Association. Retrieved from http://content.apa.org/books/10614-009 
Watson, N. F., Badr, M. S., Belenky, G., Bliwise, D. L., Buxton, O. M., Buysse, D., ... Tasali, E. (2015). Recommended Amount of Sleep for a Healthy Adult: A Joint Consensus Statement of the American Academy of Sleep Medicine and Sleep Research Society. Sleep, 38(6), 843-844. https://doi.org/10.5665/sleep.4716

Weathers, F. W., Huska, J. A., \& Keane, T. M. (1991). PCL-C for DSM-IV. Boston, Mass.: National Center for PTSD -- Behavioral Science Division.

Weilburg, J. B., Stakes, J. W., \& Roth, T. (2008). Chapter 22: Sleep Disorders. In T. Stern \& J. F. Rosenbaum (Eds.), Massachusetts General Hospital Comprehensive Clinical Psychiatry (pp. 285-301). Mosby, Inc., an affiliate of Elsevier, Inc.

Westat. (2010). National Survey of Veterans, Active Duty Service Members, Demobilized National Guard and Reserve Members, Family Members, and Surviving Spouses (Final No. GS-23F-8144H) (p. 350). Washington, D.C: Department of Veterans Affairs.

Westman, M. (2001). Stress and Strain Crossover. Human Relations, 54(6), 717-751. https://doi.org/10.1177/0018726701546002

Weston, C. S. E. (2014). Posttraumatic Stress Disorder: A Theoretical Model of the Hyperarousal Subtype. Frontiers in Psychiatry, 5. https://doi.org/10.3389/fpsyt.2014.00037

Wilcox, S. L., Oh, H., Redmond, S. A., Chicas, J., Hassan, A. M., Lee, P.-J., \& Ell, K. (2015). A scope of the problem: Post-deployment reintegration challenges in a National Guard Unit. Work (Reading, Mass.), 50(1), 73-83. https://doi.org/10.3233/WOR-141935 
Winkleby, M. A., Jatulis, D. E., \& Fortmann, S. P. (1992). Socioeconomic status and health: how eduction, income, and occupation contribute to risk factors for cardiovascular disease. American Journal of Public Health, 82(6), 816-820.

Wolf, E. J., Mitchell, K. S., Koenen, K. C., \& Miller, M. W. (2014). Combat exposure severity as a moderator of genetic and environmental liability to post-traumatic stress disorder. Psychological Medicine, 44(07), 1499-1509. https://doi.org/10.1017/S0033291713002286

Xu, K. (2012). Multiple group measurement invariance analysis in Lavaan. University of Cambridge.

Yehuda, R., Hoge, C. W., McFarlane, A. C., Vermetten, E., Lanius, R. A., Nievergelt, C. M., ... Hyman, S. E. (2015). Post-traumatic stress disorder. Nature Reviews. Disease Primers, 1, 15057. https://doi.org/10.1038/nrdp.2015.57

Yoon, J., \& Lim, JC. (1999). Organizational Support in the Workplace: The Case of Korean Hospital Employees. Human Relations, 52(7), 923-945.

Yosick, T., Bates, M., Moore, M., Crowe, C., Phillips, J., \& Davison, J. (2012). A Review of Post-Deployment Reintegration: Evidence, Challenges, and Strategies for Program Development. Deparment of Defense.

Zapf, D., Dorman, C., \& Frese, M. (1996). Longitudinal studies in organizational stress research: A review of the literature with reference to methodological issues. Journal of Occupational Health Psychology, 1(2), 145:169.

Zavis, A. (2012, November 23). National Guard soldiers and airmen face unemployment crisis. Los Angeles Times. Los Angeles, CA. Retrieved from 
Combat Experiences, Iso-strain, and Sleep Quality Affect PTSS http://articles.latimes.com/2012/nov/23/local/la-me-national-guard-employment20121124

Zoroya, G. (2013, April 6). Recent war vets face hiring obstacle: PTSD bias. USA TODAY. Retrieved from http://www.usatoday.com/story/news/nation/2013/04/06/recent-war-vets-facehiring-obstacle-ptsd-bias/2057857/ 